\title{
Branching out
}

Citation for published version (APA):

Verzaal, N. J. (2021). Branching out: CRT beyond current concepts. [Doctoral Thesis, Maastricht University]. Maastricht University. https://doi.org/10.26481/dis.20210510nv

Document status and date:

Published: 01/01/2021

DOI:

10.26481/dis.20210510nv

Document Version:

Publisher's PDF, also known as Version of record

\section{Please check the document version of this publication:}

- A submitted manuscript is the version of the article upon submission and before peer-review. There can be important differences between the submitted version and the official published version of record.

People interested in the research are advised to contact the author for the final version of the publication, or visit the DOI to the publisher's website.

- The final author version and the galley proof are versions of the publication after peer review.

- The final published version features the final layout of the paper including the volume, issue and page numbers.

Link to publication

\footnotetext{
General rights rights.

- You may freely distribute the URL identifying the publication in the public portal. please follow below link for the End User Agreement:

www.umlib.nl/taverne-license

Take down policy

If you believe that this document breaches copyright please contact us at:

repository@maastrichtuniversity.nl

providing details and we will investigate your claim.
}

Copyright and moral rights for the publications made accessible in the public portal are retained by the authors and/or other copyright owners and it is a condition of accessing publications that users recognise and abide by the legal requirements associated with these

- Users may download and print one copy of any publication from the public portal for the purpose of private study or research.

- You may not further distribute the material or use it for any profit-making activity or commercial gain

If the publication is distributed under the terms of Article $25 \mathrm{fa}$ of the Dutch Copyright Act, indicated by the "Taverne" license above, 
Nienke Juliëtte Verzaal

\section{Branching out}

CRT beyond current concepts

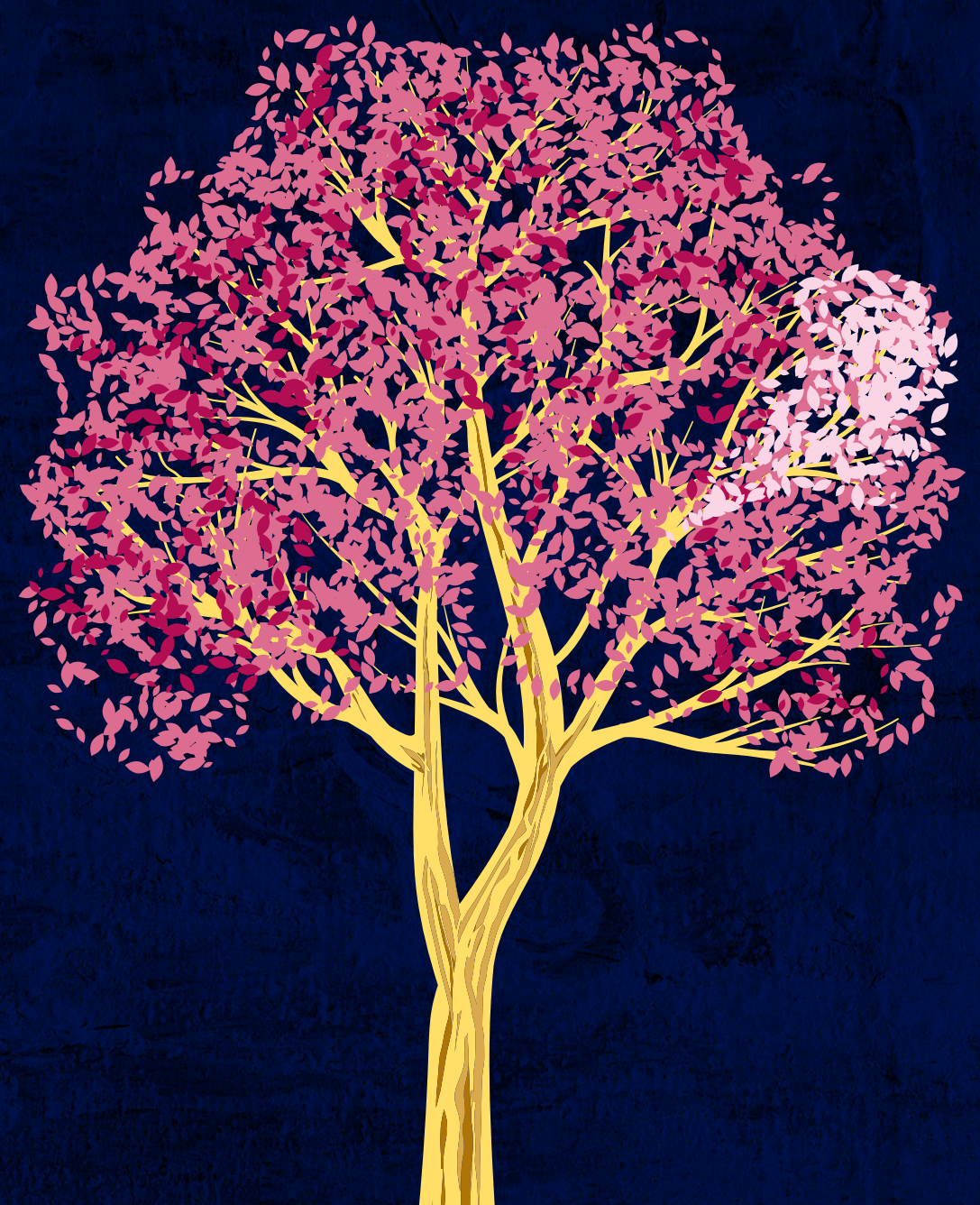





\section{Branching out}

CRT beyond current concepts

Nienke Juliëtte Verzaal 


\section{Colophon}

Cover design: James Jardine | www.jamesjardine.nl

Layout:

James Jardine | www.jamesjardine.nl

Print:

Ridderprint | www.ridderprint.nl

ISBN:

978-94-6416-520-3

Copyright @ 2021 Nienke J. Verzaal. All rights reserved. 


\title{
Branching out
}

\author{
CRT beyond current concepts
}

\author{
PROEFSCHRIFT \\ ter verkrijging van de graad van doctor aan de Universiteit Maastricht, \\ op gezag van de Rector Magnificus, Prof. dr. Rianne M. Letschert \\ volgens het besluit van het College van Decanen, \\ in het openbaar te verdedigen \\ op maandag 10 mei 2021 om 13.00 uur \\ door \\ Nienke Juliëtte Verzaal \\ Geboren op 23 maart 1992 \\ te Haarlem
}




\section{Promotores}

Prof. dr. F.W. Prinzen

Prof. dr. T. Delhaas

\section{Copromotor}

Dr. C.J.M. van Deursen

\section{Beoordelingscommissie}

Prof. dr. U. Schotten (voorzitter)

Prof. dr. H.P. Brunner-La Rocca

Dr. R. Coronel (Amsterdam Universitair Medische Centra)

Prof. dr. P.G.A. Volders

Prof. dr. M.A. Vos (Universitair Medisch Centrum Utrecht)

Financial support from Hart en vaat onderzoekfonds Limburg/Health Foundation

Limburg (Maastricht) for the publication of this thesis is gratefully acknowledged. 


\section{Table of contents}

Chapter 1 General introduction 7

Chapter 2 Pathobiology of cardiac dyssynchrony and resynchronization 23 therapy

Chapter 3 Time course of repolarization changes after start of cardiac 53 resynchronization therapy

Chapter 4 Relation between repolarization remodelling and response to 77 cardiac resynchronization therapy

Chapter 5 Can CRT beneficially impact cardiac function in the presence of structural mitral regurgitation?

Chapter 6 Exploring the cause of conduction delays in patients with repaired Tetralogy of Fallot

Chapter 7 General discussion

$\begin{array}{ll}\text { Summary } & 147\end{array}$

Impact 155

Acknowledgements 161

$\begin{array}{ll}\text { About the author } & 167\end{array}$ 


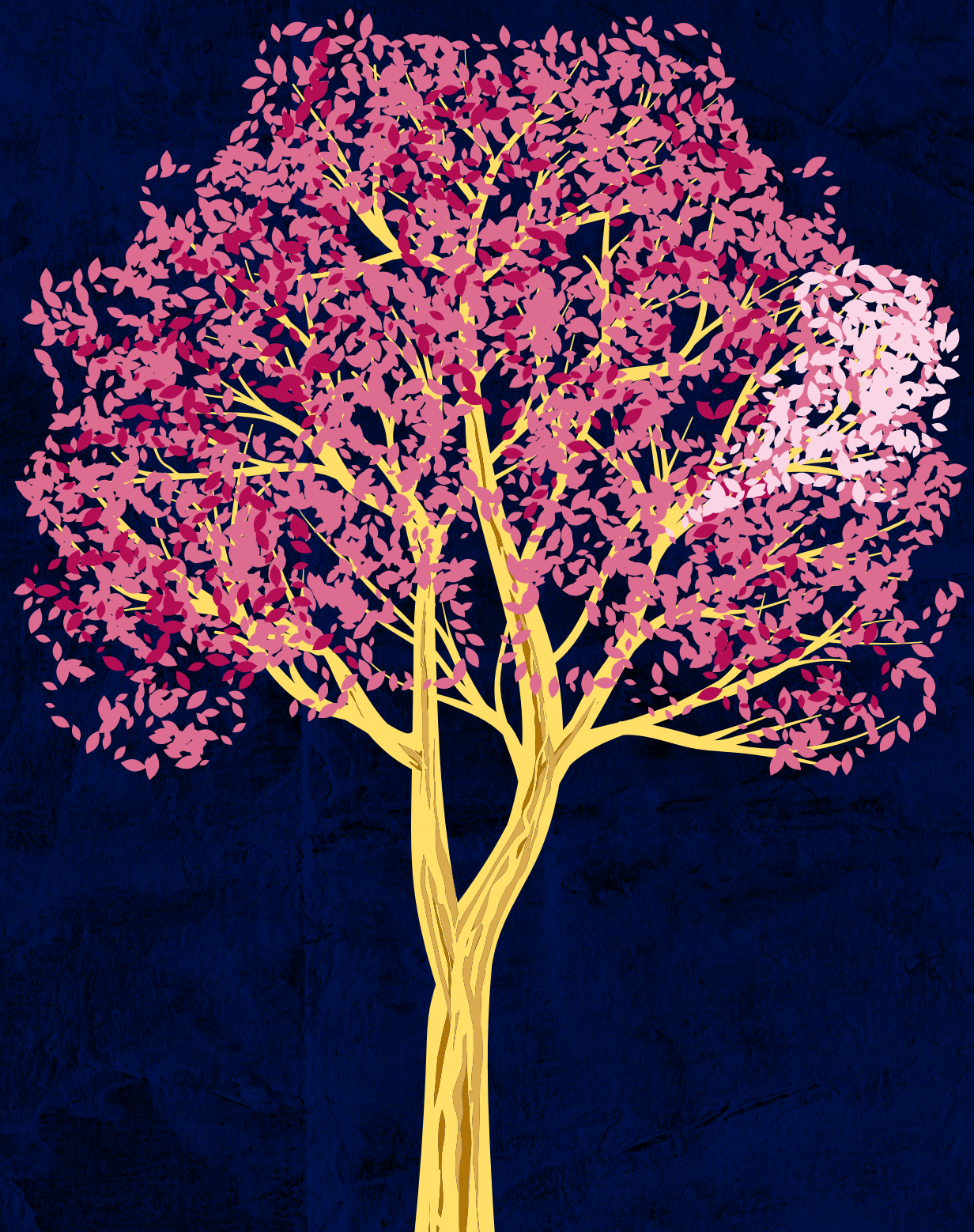


1

General introduction 

The heart consists of four chambers: the left atrium and ventricle (LA and LV, respectively) and the right atrium and ventricle (RA and $R V$, respectively)(Figure 1). Deoxygenated blood from the systemic circulation enters the RA, moves through the tricuspid valve to the RV and then, through the pulmonary valve, to the lungs. After oxygenation, the blood moves to the LA and through the mitral valve, to enter the LV. Subsequently, it passes through the aortic valve and re-enters the systemic circulation.

Cardiac contraction is initiated by an electrical impulse. All chambers are electrically activated in an appropriate sequence through the cardiac conduction system (Figure 1). Normal cardiac activation starts in the sinoatrial node, in the RA. The activation wave then travels across both atria and, subsequently, through the atrioventricular node, which slows down conduction. Thereafter, the impulses continue into the His bundle, the first section of the specialized, highvelocity conduction system of the ventricles. The His bundle then splits into a left and a right bundle branch. Conduction through these branches enables synchronized and fast activation of both LV and RV.

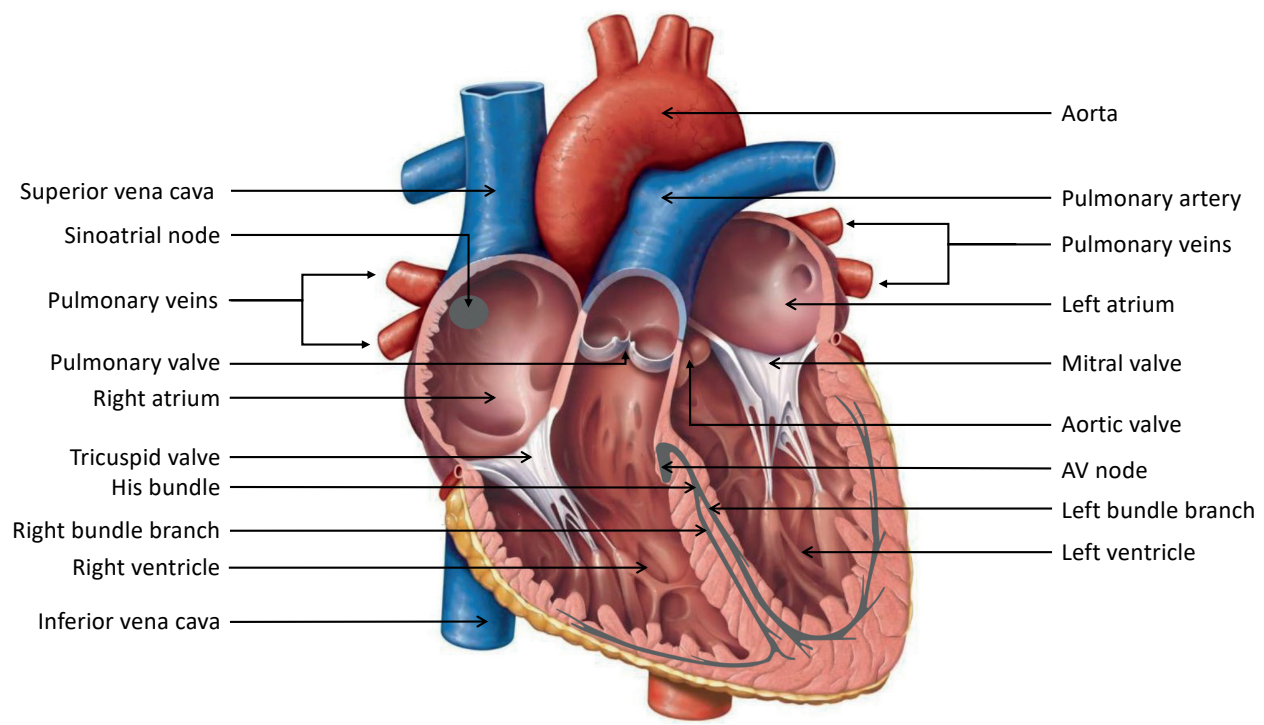

FIGURE 1. An overview of cardiac anatomy. AV node = atrioventricular node. Adapted from Marieb, EN and Keller, SM. Essentials of Human Anatomy and Physiology, $12^{\text {th }}$ edition (2017). 


\section{Bundle branch block}

If one of the bundle branches is damaged, left bundle branch block (LBBB) or right bundle branch block (RBBB) occurs, and activation of the affected ventricle has to come about by means of conduction through the working myocardium. This type of conduction is considerably slower ( $0.5 \mathrm{~m} / \mathrm{sec}$, while conduction through the His bundle occurs at 1-3 m/ $\left.\mathrm{sec}^{1,2}\right)$, meaning that both activation and contraction of the affected ventricle (and in particular, its free wall) are delayed ${ }^{3}$.

In the case of LBBB the early-activated septum contracts prior to the other areas. Therefore, the septum contracts against a relatively low pressure in the LV, and performs little work 4 . Areas that are activated late, on the other hand, experience pre-stretch (caused by contraction of areas that are activated early), leading to activation of the Frank-Starling mechanism and stronger regional contraction. This, in turn, increases local work in late-activated regions ${ }^{4}$. In the case of RBBB, a similar cardiac activation pattern occurs in the RV5.

Such abnormal contraction patterns also lead to a loss of efficiency of contraction, because early-activated areas waste energy stretching late-activated areas, and vice versa ${ }^{4,6}$.

The dyssynchronous activation pattern has multiple consequences for the myocardium at the molecular and cellular level (see chapter 2 for more details). Broadly, we can split these remodelling consequences into three categories: structural (e.g. fibrosis and hypertrophy), electrical (e.g. changes in expression of connexin 43 and ion channels related to depolarization and repolarization) and contractile remodelling (e.g. changes in calcium handling and contractile proteins $)^{4}$. At the organ level, these changes become apparent as, for example, asymmetric hypertrophy and cardiac dilatation ${ }^{4}$.

Over time, all aforementioned molecular and cellular derangements lead to worsened cardiac function and more severe conduction delay, starting a vicious circle that can culminate in heart failure $^{4}$ (Figure 2, see chapter 2 for more details). 


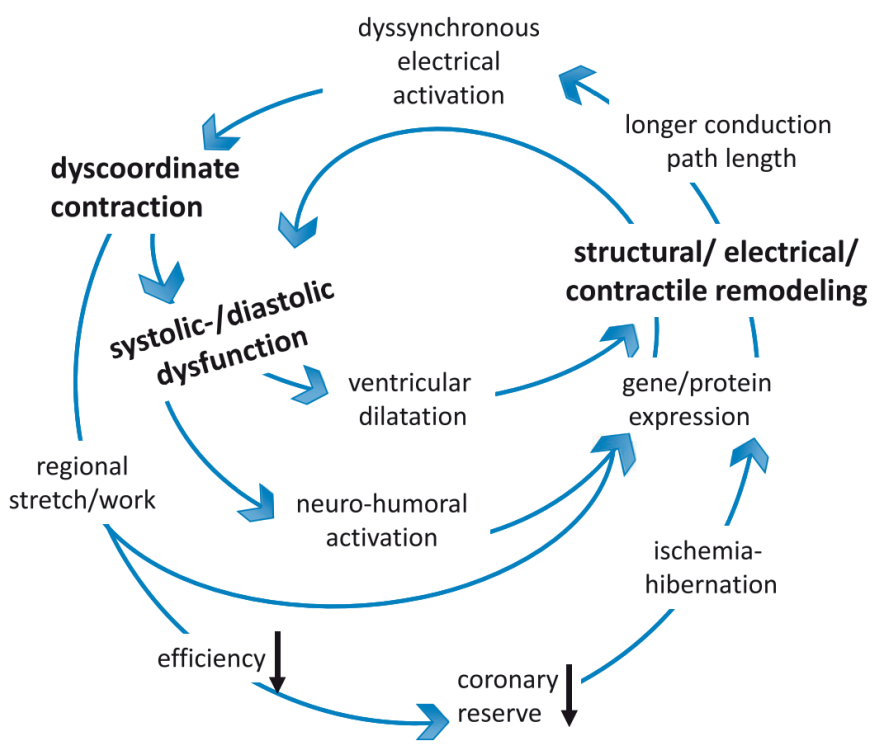

FIGURE 2. Overview of the vicious circle of dyssynchrony. Reproduced from ${ }^{4}$.

\section{Cardiac resynchronization therapy: current concepts and unexplored territories}

Cardiac resynchronization therapy (CRT) may reverse the vicious circle of dyssynchrony in hearts with $\mathrm{LBBB}$, and possibly also $\mathrm{RBBB}^{7,8}$. This treatment involves implanting a pacemaker and placing the electrodes (leads) in the RA, the RV and an epicardial vein on the LV. Ventricular contraction is resynchronized by (almost) simultaneous stimulation from both ventricular leads. Such resynchronization can cause both acute and long-term improvements in cardiac function, which in turn lead to lower risk of heart failure hospitalisations and death, in particular in patients with heart failure and LBBB $^{9-11}$.

CRT benefit can be explained by several types of favourable cardiac remodelling. For instance, improved calcium handling can have positive effects on both electrical and contractile properties ${ }^{4}$. At the organ level, a reduction in asymmetric hypertrophy and ventricular volumes

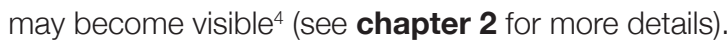

Over the last two decades CRT has become an accepted treatment for patients with heart failure and LBBB. However, several aspects are less well understood. First of all, while the effects of CRT on cardiac depolarization have been studied in considerable detail, much less 
is known about the effects of CRT on repolarization. Furthermore, much is yet to be learned about the efficacy of CRT in structural heart disease, such as mitral regurgitation or congenital heart disease.

\section{Repolarization and CRT}

The effects of CRT on cardiac electrical activation (depolarization) have been studied extensively. However, several studies have found that CRT may also affect the electrical "deactivation" (repolarization) of the heart ${ }^{12,13}$. The effect of CRT on repolarization is particularly important since dispersion of repolarization has been linked to a higher risk of cardiac arrhythmias. The role of CRT in this matter seems somewhat ambiguous. An increase in repolarization dispersion with concomitant arrhythmia development after start of CRT has been reported by some ${ }^{14}$, while other studies examining patients after an upgrade from implantable cardioverter defibrillator to CRT did not find an increased occurrence of arrhythmias ${ }^{15,16}$. Additionally, patients who respond to CRT have a lower risk of developing arrhythmias, suggesting that beneficial electrical remodelling may occur ${ }^{17,18}$.

Regardless of the exact mechanism, alterations in cardiac activation patterns have indeed been found to induce electrical remodelling. These changes can be observed by studying the T wave of the electrocardiogram (ECG) and vectorcardiogram (VCG, Figure 3), both during prolonged $\mathrm{CRT}^{12,19}$ and during prolonged dyssynchrony (brought about by $\mathrm{RV}$ pacing or LBBB $^{20-23}$ ). Shortly after onset of dyssynchrony an increase in T wave area and amplitude has been found ${ }^{21,23}$, which is indicative of a rise in dispersion of repolarization ${ }^{24}$. Subsequently, $T$ wave area and amplitude decrease if dyssynchrony persists ${ }^{21-23}$.

Further evidence for electrical remodelling is observed when pacing from the RV is switched off and dyssynchrony is briefly halted (Figure 4). In that case, the opposite occurs: T wave size increases relative to its baseline value ${ }^{21}$. Moreover, its direction deviates from its initial orientation during normal activation, despite the fact that the pacemaker has been switched off $^{21}$. Because the direction of the T wave follows the direction of the paced QRS complex this phenomenon has been named cardiac or T wave memory ${ }^{21,25}$.

In the case of CRT (so resynchronization) similar phenomena can be observed, including an acute increase in $\mathrm{T}$ area when the device is switched off $^{12,19}$. However, until now, the time course of these electrical remodelling processes has not been studied in detail, nor has the relation with contractile remodelling been elucidated. 


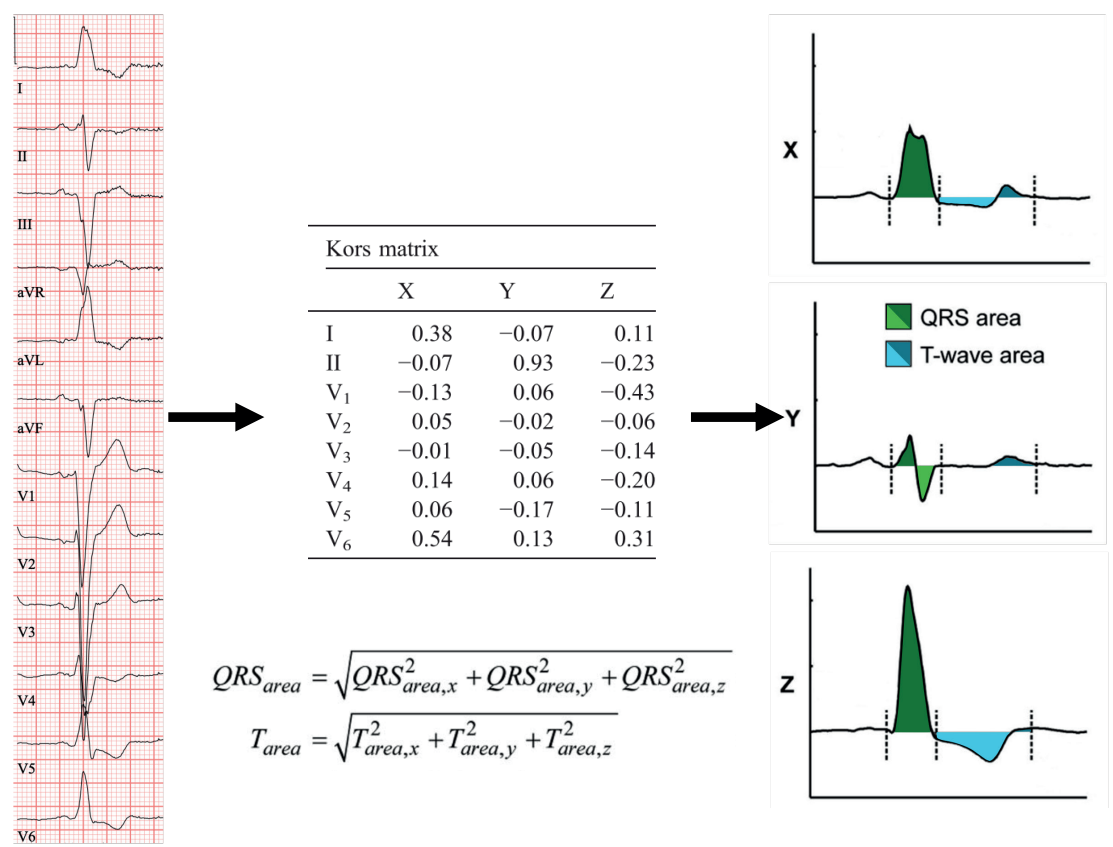

FIGURE 3. An electrocardiogram (ECG, left column) can be converted (using the Kors matrix, central table) to a vectorcardiogram (VCG, right column). A VCG expresses cardiac depolarization and repolarization over time in three dimensions. In this way, a VCG provides information about both direction and magnitude of cardiac electrical wavefronts. Light green and blue areas are subtracted from dark green and blue areas (right column), and total QRS and $T$ areas are calculated using the formulas at the bottom. Adapted from ${ }^{46}$ and ${ }^{47}$.

A Sinus rhythm - Control

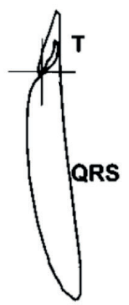

B

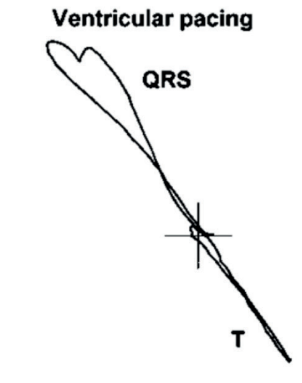

crosshair size $=0.5 \times 0.5 \mathrm{mV}$

frontal plane
C T wave progression

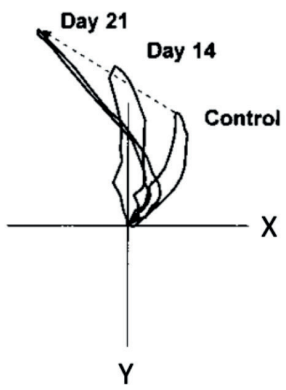




\section{Mitral regurgitation and CRT}

The mitral valve consists of a ring of connective tissue (annulus) that encircles two leaflets. The leaflets are connected to papillary muscles close to the apex of the LV by chords of connective tissue, known as the chordae tendineae. Normally, the valve closes due to pressure build-up in the contracting LV while the papillary muscles pull on the chordae tendineae to keep the leaflets from protruding into the $L A$. Thus, blood is prevented from regurgitating into the $L A$ (Figure $5 \mathrm{~A}$ and $\mathrm{B}$ ).

However, many patients who are candidates for CRT have some degree of mitral regurgitation (MR). This may be structural MR, caused by structural heart disease such as damage to the chordae tendineae (Figure 5C). On the other hand, LBBB can also lead to functional MR due to several mechanisms. For instance, less efficient LV contraction leads to a lower closing force on the mitral valve leaflets ${ }^{26}$. Moreover, the mitral valve annulus shape and the position of the papillary muscles may be abnormal due to ventricular dyssynchrony or dilatation, which may create a regurgitant orifice within the valve by impairing coaptation (closure) of the mitral valve leaflets ${ }^{26}$. Lastly, ill-timed contraction of the LA and LV can lead to MR during cardiac relaxation ${ }^{26}$.

A

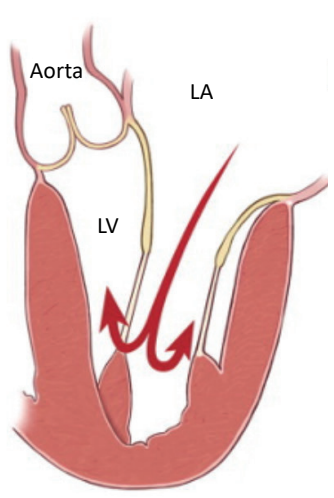

Diastole

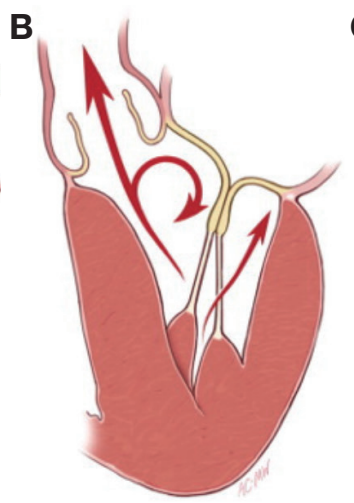

Systole
C

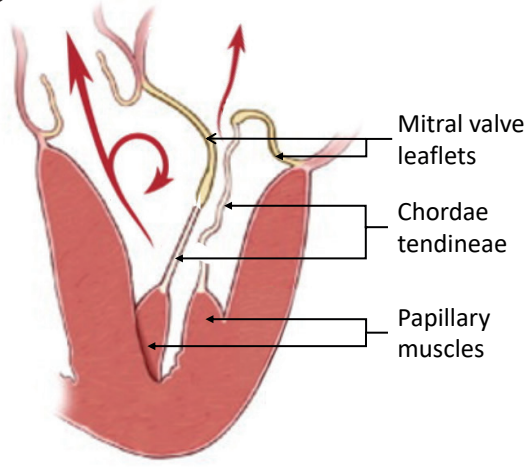

MR during systole

FIGURE 5. A. During ventricular relaxation (diastole), blood (red arrows) enters the LV from the LA. B. During LV contraction (systole), the mitral valve closes due to the build-up of pressure by the LV and chordae tendineae keep the leaflets in place. Blood moves into the aorta. C. If chordae tendineae are ruptured, they cannot keep the mitral valve leaflet in place during systole, and blood regurgitates into the LA during LV contraction. MR = mitral regurgitation, LA = left atrium, LV = left ventricle. Adapted from Carpentier A, Adams DH and Filsoufi F. Carpentier's Reconstructive Valve Surgery, $1^{\text {st }}$ edition (2010). 
Conceptually, the underlying cause of MR is important, since different causes may require different treatments ${ }^{27}$. CRT may be able to correct functional MR through several mechanisms ${ }^{26}$. For example, the more efficient cardiac contraction can increase the force with which the mitral valve leaflets are closed ${ }^{28}$ and resynchronization of the papillary muscles may result in improved positioning of the valve leaflets, optimizing valve closure ${ }^{29}$. Additionally, an improvement in the relative timing of atrial and ventricular contraction ${ }^{30}$ or a reduction in the size of the mitral orifice may reduce $\mathrm{MR}^{26}$. Beneficial effects can be observed both acutely and long-term, since reverse remodelling caused by CRT can lead to improved coaptation of the mitral valve leaflets through smaller LV volumes and better alignment of papillary muscles and chordae tendineae ${ }^{26}$.

However, in some patients CRT is not accompanied by improvement of MR. Such persistent MR is associated with poorer long-term cardiac function ${ }^{31,32}$ and (event-free) survival29,32,33. Nonetheless, even in the case of persistent MR CRT may be beneficial ${ }^{29,34,35}$. Further studies into the benefit of CRT in the presence of structural MR are needed to expand our understanding of these phenomena.

\section{Tetralogy of Fallot}

Recently, in addition to its beneficial effects on the LV in LBBB, CRT has also been explored in patients with $\mathrm{RBBB}^{36-38}$. A particular situation in which this has been attempted is after surgical repair of Tetralogy of Fallot (ToF). ToF is the most common type of cyanotic congenital heart disease, occurring in approximately 1 in 3600 live births ${ }^{39}$.

In patients with ToF, four abnormalities are present in the heart: ventricular septal defect (VSD), aorta overriding the VSD, right ventricular outflow tract obstruction (RVOTO) and right ventricular hypertrophy (Figure 6). If the RVOTO is sufficiently severe, blood is shunted from the RV into the LV, leading to reduced pulmonary blood flow and cyanosis, which is potentially life-threatening ${ }^{40}$.

During surgery, which is usually performed at a very young age, the VSD is closed with a patch and the RVOTO is resected, nowadays while preserving the integrity of the pulmonary valve as much as possible ${ }^{39}$. However, after surgery, a combination of pulmonary stenosis and regurgitation is often present. Furthermore, RBBB commonly occurs, either proximally, due to VSD closure, or more distally, due to the RVOTO resection or ventriculotomy ${ }^{41,42}$. 


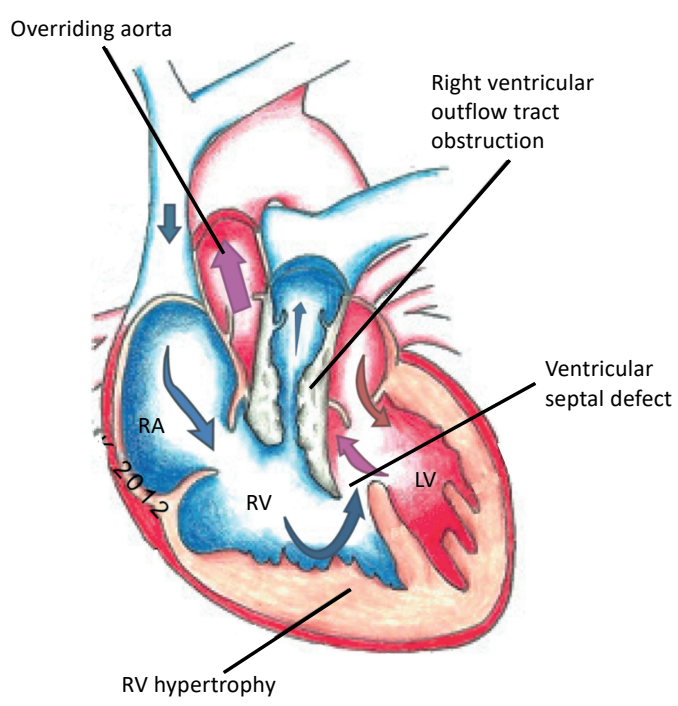

FIGURE 6. The four cardiac defects found in patients with Tetralogy of Fallot. Blood with little oxygen (blue arrows) is shunted into the LV through the ventricular septal defect. $R A=$ right atrium, $R V=$ right ventricle, $L V=$ left ventricle. Adapted from ${ }^{40}$.

This combination of RV pressure overload, volume overload and RBBB can lead to pathological $\mathrm{RV}$ remodelling and, eventually, to RV failure and cardiac arrhythmias ${ }^{40,42}$. Imaging and electrical mapping studies have revealed that patients with repaired ToF (rToF) show both mechanical and electrical dyssynchrony, suggesting that resynchronization of the RV could be beneficial ${ }^{42-44}$. However, CRT requires normal myocardial conduction properties, since the presence of scar can reduce its efficacy ${ }^{45}$. Conversely, presence of conduction blocks in the RV might hamper the benefit of CRT. Whether this phenomenon also occurs in patients with rToF has not yet been investigated in detail.

\section{Aims of the thesis}

The bulk of CRT research has been conducted in the context of resynchronization of depolarization during LBBB. As the title of this thesis implies, our aim is to investigate aspects of CRT beyond the well-known resynchronization of activation and contraction, both in terms of its application and its consequences. 
We aimed to answer the following questions:

1) What is the time course of repolarization changes following CRT, and how does dispersion of repolarization change after CRT?

2) Is there a link between repolarization changes and response to CRT?

3) May CRT still be beneficial in the context of structural MR?

4) Do myocardial conduction properties in patients suffering from rToF suggest a favourable substrate for CRT?

\section{Outline of the thesis}

In chapter 2 we review the pathobiology of dyssynchrony and its correction by resynchronization, covering both clinical aspects and molecular mechanisms.

In chapter $\mathbf{3}$ we study the time course of repolarization changes in relation to contractile remodelling by analysing serial ECGs and echocardiograms from patients who received CRT. Moreover, we explore the consequences of this repolarization remodelling for dispersion of repolarization using a computer model of cardiac depolarization and repolarization.

In chapter 4 we investigate whether repolarization remodelling is more prominent in CRT responders (patients showing a large increase in LV ejection fraction) than in non-responders, by examining ECGs and echocardiograms recorded prior to CRT implantation and after approximately 6 months of treatment.

In chapter 5 we describe the acute effects of CRT in two dog models: one of MR+LBBB and one of isolated LBBB. We compare the haemodynamic effects (effects related to blood flow and pressure) of various pacing modes in these animal models. Moreover, we assess changes in cardiac function over time during both LBBB and MR+LBBB.

Chapter 6 examines myocardial conduction properties in a small group of patients with rToF and in several non-rToF patients. These patients underwent epicardial electrical mapping (on the outside of the heart) of both ventricles and endocardial mapping (on the inside of the heart) of the RV, during medically indicated cardiac surgery. We investigated whether structural conduction blocks occurred by comparing activation patterns recorded during sinus rhythm and during ventricular pacing.

The final section of this thesis (chapter 7) is a general discussion, in which our findings are discussed in a broader scientific perspective. Moreover, we describe the potential scientific and clinical impact of our work. 


\section{References}

1. Durrer D, Dam RT van, Freud GE, Janse MJ, Meijler FL, Arzbaecher RC. Total excitation of the isolated human heart. Circulation 1970;41:899-912.

2. Boyden PA, Hirose M, Dun W. Cardiac Purkinje cells. Hear Rhythm 2010;7:127-35.

3. Vernooy K, Verbeek XAAM, Peschar M, Crijns HJGM, Arts T, Cornelussen RNM, et al. Left bundle branch block induces ventricular remodelling and functional septal hypoperfusion. Eur Heart $J$ 2005;26:91-8.

4. Nguyên UC, Verzaal NJ, Nieuwenhoven FA van, Vernooy K, Prinzen FW. Pathobiology of cardiac dyssynchrony and resynchronization therapy. Europace 2018;20:1898-909.

5. Janoušek J, Kovanda J, Ložek M, Tomek V, Vojtovič P, Gebauer R, et al. Pulmonary Right Ventricular Resynchronization in Congenital Heart Disease: Acute Improvement in Right Ventricular Mechanics and Contraction Efficiency. Circ Cardiovasc Imaging 2017;10:e006424.

6. Vecera J, Penicka M, Eriksen M, Russell K, Bartunek J, Vanderheyden M, et al. Wasted septal work in left ventricular dyssynchrony: A novel principle to predict response to cardiac resynchronization therapy. Eur Heart J Cardiovasc Imaging 2016;17:624-32.

7. Brignole M, Auricchio A, Baron-Esquivias G, Bordachar P, Boriani G, Breithardt O-A, et al. 2013 ESC Guidelines on cardiac pacing and cardiac resynchronization therapy. Eur Heart J 2013;34:2281329.

8. Auricchio A, Lumens J, Prinzen FW. Does cardiac resynchronization therapy benefit patients with right bundle branch block: cardiac resynchronization therapy has a role in patients with right bundle branch block. Circ Arrhythm Electrophysiol 2014;7:532-42.

9. Goldenberg I, Kutyifa V, Klein HU, Cannom DS, Brown MW, Dan A, et al. Survival with cardiacresynchronization therapy in mild heart failure. N Engl J Med 2014;370:1694-701.

10. Zareba W, Klein H, Cygankiewicz I, Hall WJ, McNitt S, Brown M, et al. Effectiveness of cardiac resynchronization therapy by QRS morphology in the multicenter automatic defibrillator implantation trial-cardiac resynchronization therapy (MADIT-CRT). Circulation 2011;123:1061-72.

11. Nelson GS, Berger RD, Fetics BJ, Talbot M, Spinelli JC, Hare JM, et al. Left ventricular or biventricular pacing improves cardiac function at diminished energy cost in patients with dilated cardiomyopathy and left bundle-branch block. Circulation 2000;102:3053-9.

12. Wecke L, Deursen CJM Van, Bergfeldt L, Prinzen FW. Repolarization changes in patients with heart failure receiving cardiac resynchronization therapy - Signs of cardiac memory. J Electrocardiol 2011;44:590-8.

13. Padeletti L, Fantappiè C, Perrotta L, Ricciardi G, Pieragnoli P, Chiostri M, et al. Cardiac memory in humans: Vectocardiographic quantification in cardiac resynchronization therapy. Clin Res Cardiol 2011; 100:51-6.

14. Medina-Ravell VA, Lankipalli RS, Yan G-X, Antzelevitch C, Medina-Malpica NA, Medina-Malpica $\mathrm{OA}$, et al. Effect of epicardial or biventricular pacing to prolong QT interval and increase transmural dispersion of repolarization: does resynchronization therapy pose a risk for patients predisposed to long QT or torsade de pointes? Circulation 2003;107:740-6. 
15. Thijssen J, Borleffs CJW, Delgado V, Rees JB Van, Mooyaart EAQ, Bommel RJ Van, et al. Implantable cardioverter-defibrillator patients who are upgraded and respond to cardiac resynchronization therapy have less ventricular arrhythmias compared with nonresponders. J Am Coll Cardiol 2011;58:2282-9.

16. Lin G, Rea RF, Hammill SC, Hayes DL, Brady PA. Effect of cardiac resynchronisation therapy on occurrence of ventricular arrhythmia in patients with implantable cardioverter defibrillators undergoing upgrade to cardiac resynchronisation therapy devices. Heart 2008;94:186-90.

17. Saini A, Kannabhiran M, Reddy P, Gopinathannair R, Olshansky B, Dominic P. Cardiac Resynchronization Therapy May Be Antiarrhythmic Particularly in Responders: A Systematic Review and Meta-Analysis. JACC Clin Electrophysiol 2016;2:307-16.

18. Deif B, Ballantyne B, Almehmadi F, Mikhail M, Mclntyre WF, Manlucu J, et al. Cardiac resynchronization is pro-arrhythmic in the absence of reverse ventricular remodelling: A systematic review and metaanalysis. Cardiovasc Res 2018;114:1435-44.

19. Perrotta L, Ricciardi G, Pieragnoli P, Nesti M, Pontecorboli G, Fantini F, et al. Cardiac memory in cardiac resynchronization therapy: A vectorcardiographic comparison of biventricular and left ventricular pacing. J Electrocardiol 2015;48:571-7.

20. Wecke L, Gadler F, Linde C, Lundahl G, Rosen MR, Bergfeldt L. Temporal characteristics of cardiac memory in humans: Vectorcardiographic quantification in a model of cardiac pacing. Hear Rhythm 2005;2:28-34.

21. Shvilkin A, Bojovic B, Vajdic B, Gussak I, Zimetbaum P, Josephson ME. Vectorcardiographic determinants of cardiac memory during normal ventricular activation and continuous ventricular pacing. Hear Rhythm 2009;6:943-8.

22. Shvilkin A, Bojovic B, Vajdic B, Gussak I, Ho KK, Zimetbaum P, et al. Vectorcardiographic and electrocardiographic criteria to distinguish new and old left bundle branch block. Hear Rhythm 2010;7:1085-92.

23. Engels EB, Poels TT, Houthuizen P, Jaegere PPT de, Maessen JG, Vernooy K, et al. Electrical remodelling in patients with iatrogenic left bundle branch block. Europace 2016;18:iv44-52.

24. Huysduynen BH Van, Swenne CA, Draisma HHM, Antoni ML, Vooren H Van De, Wall EE Van Der, et al. Validation of ECG indices of ventricular repolarization heterogeneity: A computer simulation study. J Cardiovasc Electrophysiol 2005;16:1097-103.

25. Rosen MR, Bergfeldt L. Cardiac memory: The slippery slope twixt normalcy and pathology. Trends Cardiovasc Med 2015;25:687-96.

26. Spartera M, Galderisi M, Mele D, Cameli M, D'Andrea A, Rossi A, et al. Role of cardiac dyssynchrony and resynchronization therapy in functional mitral regurgitation. Eur Heart $J$ Cardiovasc Imaging 2016;17:471-80.

27. Packer M, Grayburn PA. Contrasting Effects of Pharmacological, Procedural, and Surgical Interventions on Proportionate and Disproportionate Functional Mitral Regurgitation in Chronic Heart Failure. Circulation 2019;140:779-89.

28. Breithardt OA, Sinha AM, Schwammenthal E, Bidaoui N, Markus KU, Franke A, et al. Acute effects of cardiac resynchronization therapy on functional mitral regurgitation in advanced systolic heart failure. J Am Coll Cardiol 2003;41:765-70. 
29. Bartko PE, Arfsten H, Heitzinger G, Pavo N, Strunk G, Gwechenberger M, et al. Papillary Muscle Dyssynchrony-Mediated Functional Mitral Regurgitation: Mechanistic Insights and Modulation by Cardiac Resynchronization. JACC Cardiovasc Imaging 2019;12:1728-37.

30. Salden FCWM, Kutyifa V, Stockburger M, Prinzen FW, Vernooy K. Atrioventricular dromotropathy: evidence for a distinctive entity in heart failure with prolonged PR interval? Europace 2018;20:106777.

31. Mihos CG, Santana O, Yucel E, Capoulade R, Upadhyay GA, Orencole MP, et al. The effects of cardiac resynchronization therapy on left ventricular and mitral valve geometry and secondary mitral regurgitation in patients with left bundle branch block. Echocardiography 2019;36:1450-8.

32. Bijl P van der, Khidir M, Ajmone Marsan N, Delgado V, Leon MB, Stone GW, et al. Effect of Functional Mitral Regurgitation on Outcome in Patients Receiving Cardiac Resynchronization Therapy for Heart Failure. Am J Cardiol 2019;123:75-83.

33. Verhaert D, Popović ZB, De S, Puntawangkoon C, Wolski K, Wilkoff BL, et al. Impact of mitral regurgitation on reverse remodeling and outcome in patients undergoing cardiac resynchronization therapy. Circ Cardiovasc Imaging 2012;5:21-6.

34. John Sutton M St., Ghio S, Plappert T, Tavazzi L, Scelsi L, Daubert C, et al. Cardiac resynchronization induces major structural and functional reverse remodeling in patients with New York heart association class I/II heart failure. Circulation 2009;120:1858-65.

35. Biase L Di, Auricchio A, Mohanty P, Bai R, Kautzner J, Pieragnoli P, et al. Impact of cardiac resynchronization therapy on the severity of mitral regurgitation. Europace 2011;13:829-38.

36. Thambo J, Santos P Dos, Guillebon M De, Roubertie F, Labrousse L, Sacher F, et al. Biventricular stimulation improves right and left ventricular function after tetralogy of Fallot repair: acute animal and clinical studies. Hear Rhythm 2010;7:344-50.

37. Plymen CM, Finlay M, Tsang V, O'leary J, Picaut N, Cullen S, et al. Haemodynamic consequences of targeted single- and dual-site right ventricular pacing in adults with congenital heart disease undergoing surgical pulmonary valve replacement. Europace 2015;17:274-80.

38. Vojtovič P, Kučera F, Kubuš P, Gebauer R, Matějka T, Tláskal $T$, et al. Acute right ventricular resynchronization improves haemodynamics in children after surgical repair of tetralogy of Fallot. Europace 2018;20:323-8.

39. Apitz C, Webb GD, Redington AN. Tetralogy of Fallot. Lancet 2009;374:1462-71.

40. Bove $T$, François $K$, Wolf $D$ de. New insights into the surgical management of Tetralogy of Fallot: Physiological fundamentals and clinical relevance. Curr Pediatr Rev 2015;11:72-86.

41. Horowitz LN, Alexander JA, Edmunds LH. Postoperative right bundle branch block: identification of three levels of block. Circulation 1980;62:319-28.

42. Motonaga KS, Dubin AM. Cardiac resynchronization therapy for pediatric patients with heart failure and congenital heart disease: A reappraisal of results. Circulation 2014;129:1879-91.

43. Uebing A, Gibson DG, Babu-Narayan S V., Diller GP, Dimopoulos K, Goktekin O, et al. Right ventricular mechanics and QRS duration in patients with repaired tetralogy of Fallot: Implications of infundibular disease. Circulation 2007;116:1532-9. 
44. Thambo J-B, Guillebon M De, Xhaet O, Santos P Dos, Roubertie F, Labrousse L, et al. Biventricular pacing in patients with Tetralogy of Fallot: non-invasive epicardial mapping and clinical impact. Int $J$ Cardiol 2013;163:170-4.

45. Bleeker GB, Kaandorp TAM, Lamb HJ, Boersma E, SteendijkP, Roos A De, et al. Effect of posterolateral scar tissue on clinical and echocardiographic improvement after cardiac resynchronization therapy. Circulation 2006;113:969-76.

46. Schreurs CA, Algra AM, Man SC, Cannegieter SC, Wall EE van der, Schalij MJ, et al. The spatial QRS-T angle in the Frank vectorcardiogram: accuracy of estimates derived from the 12-lead electrocardiogram. J Electrocardiol 2010;43:294-301.

47. Engels EB, Végh EM, Deursen CJM Van, Vernooy K, Singh JP, Prinzen FW. T-wave area predicts response to cardiac resynchronization therapy in patients with left bundle branch block. J Cardiovasc Electrophysiol 2015;26:176-83.

48. Shvilkin A, Danilo P, Wang J, Burkhoff D, Anyukhovsky EP, Sosunov EA, et al. Evolution and Resolution of Long-term Cardiac Memory. Circulation 1998;97:1810-7. 


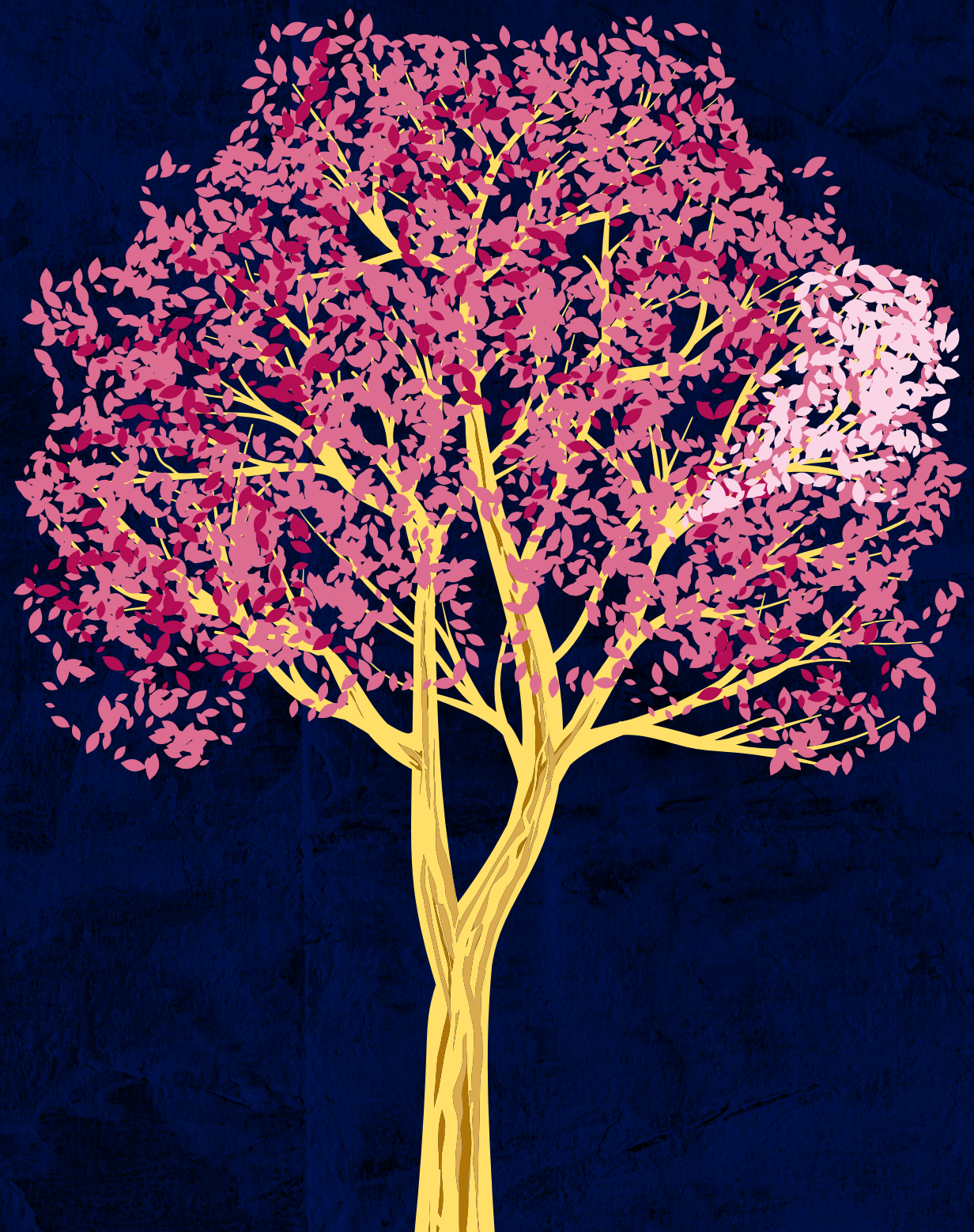




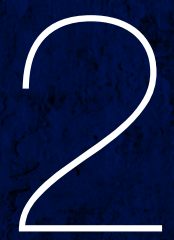

\section{Pathobiology of cardiac dyssynchrony and resynchronization therapy}

Uyên Châu Nguyên ${ }^{1 \star}$, Nienke J. Verzaal ${ }^{2 \star}$, Frans A. van Nieuwenhoven², Kevin Vernooy ${ }^{1}$ and Frits W. Prinzen ${ }^{2}$

${ }^{1}$ Department of Cardiology, Maastricht University Medical Centre, Maastricht, The Netherlands

${ }^{2}$ Department of Physiology, Maastricht University, Maastricht, The Netherlands

* These authors contributed equally.

Europace 2018;20:1898-909 


\section{Abstract}

Synchronous ventricular electrical activation is a prerequisite for adequate left ventricular (LV) systolic function. Conduction abnormalities such as left bundle branch block (LBBB), and ventricular pacing lead to a dyssynchronous electrical activation sequence, which may have deleterious consequences.

The present review attempts to connect the various processes involved in the development of "dyssynchronopathy" and its correction by cardiac resynchronization therapy (CRT). Abnormal electrical impulse conduction leads to abnormal contraction, characterized by regional differences in timing as well as shortening patterns and amount of external work performed. Early-activated regions may show "wasted work" which leads to inefficient action of the entire LV. Moreover, both the development of heart failure in general and the regional differences in mechanical load lead to structural, electrical, and contractile remodelling processes. These have been demonstrated at the level of the myocardium (asymmetric hypertrophy, fibrosis, prolongation of activation and reduction in repolarization forces, decrease in LV ejection fraction), cell (gap junctional remodelling, derangement of the T-tubular structure) and molecule (underor overexpression of ion channels and contractile protein subtypes and abnormal calcium handling). The myocardial adaptations to dyssynchrony are "maladaptive". This also explains why CRT, unlike most pharmacological treatments, continues to increase its therapeutic effect over time. Finally, better understanding of all processes involved in dyssynchrony and CRT may also lead to new pharmacological agents for treating heart failure and to novel pacing strategies. 


\section{Introduction}

Proper cardiac pump function is dependent on fairly synchronous and well-distributed electrical activation. The intraventricular rapid conduction system plays a key role in this respect. The importance of this synchronous activation is illustrated by the deviations in structure and function that occur when impulse conduction becomes abnormally slow, as is the case during ventricular pacing and left bundle branch block (LBBB). Slow impulse conduction leads to large time differences in electrical activation and contraction within a ventricle and between the ventricles (intra- and interventricular dyssynchrony, respectively). The combination of all detrimental changes leading to dyssynchrony-induced cardiomyopathy can be termed "dyssynchronopathy", a disease that can be treated by cardiac resynchronization therapy (CRT). The present overview aims to review and discuss current knowledge on the pathobiology of dyssynchrony and CRT, i.e. the causes and consequences of dyssynchrony and resynchronization for structural and functional processes in the myocardium.

\section{Electrical aspects}

\section{The pathobiology of conduction abnormalities and electrical dyssynchrony}

Synchronous ventricular electrical activation is a prerequisite for adequate left ventricular (LV) function. In the healthy heart this is achieved by propagation of electrical impulses from the atrioventricular node through the fast-conducting His-Purkinje system. An additional role may be played by fast-conducting endocardial fibers, which conduction velocity is in between that of working myocardium and Purkinje fibers ${ }^{1}$. Of note, functionality of such fibers has only been demonstrated in the dog heart, the conduction system of which seems to be closest to the human heart of all non-primate mammals². "True" Purkinje fibers are characterized by a larger size ${ }^{2}$ and by overexpression of connexin $43(\mathrm{C} \times 43)^{3}$ compared to endocardial fibers. In relatively simple histological images, the subendocardial fibers in the dog heart appear uniformly oriented but otherwise similar to working myocardium ${ }^{1}$. Such fibers have not been identified in the human heart, but high resolution micro computed tomography (CT) images show dense networks of Purkinje-like fibers, which may be comparable to the fast-conducting endocardial fibers in the dog heart. In the dog heart several studies showed fast impulse conduction along the LV endocardium ${ }^{4,5}$ apparently explaining the benefit of endocardial CRT ${ }^{4,6,7}$. Although the existence of fast-conducting subendocardial fibers in the human heart is not clear, clinical studies support the evidence that endocardial CRT is at least as beneficial as epicardial CRT ${ }^{8}$. 
While the ventricular endocardium is normally electrically activated within $\sim 20 \mathrm{~ms}$, the remaining transmural propagation from endocardium to epicardium requires another $\sim 50 \mathrm{~ms}^{9}$. The Purkinje system may be damaged or bypassed during conduction abnormalities such as LBBB and ventricular pacing. Under these conditions, conduction propagates primarily through working myocardium in which the conduction velocity is almost four times slower compared to the specialized His-Purkinje system ${ }^{9}$. Additionally, the wavefront of activation changes. Propagation perpendicular to the fiber orientation is half of that of propagation parallel to the fibers ${ }^{10}$, contributing to the prolongation of total ventricular activation.

While the cause of abnormal conduction during ventricular pacing is obvious, this is much less the case during bundle branch block. Especially during LBBB, the conduction block may occur at any level in the His-Purkinje system. The wide variation in left bundle branch anatomies further complicates the diagnosis of LBBB $^{11}$. LBBB may develop suddenly when its primary blood supply is obstructed or under iatrogenic circumstances, for instance after aortic valve replacement. In the latter case, the block is obviously proximal in the left bundle branch. However, more often, LBBB is a result of slow degeneration of the conduction system due to chronic conditions affecting the myocardium ${ }^{12}$.

There is interesting and growing evidence that, in the latter case, a considerable part of LBBB patients has a proximal block. This evidence comes from the fact that His bundle pacing creates significant narrowing of the QRS complex in many patients with LBBB. Because this effect can only be explained if pacing occurred distal to the block, this LBBB should be located very proximal in the His bundle or even in the lower part of the atrioventricular (AV) node. Forty-year-old studies already pointed this out ${ }^{13,14}$, and results were explained by the theory of longitudinal dissociation of the His bundle. This theory assumes that the conducting fibers to the right and left bundle branch are histologically isolated inside the trunk. Injuring the trunk may lead to a complete AV-block or a bundle branch block ${ }^{15}$. Stimulation of the fibers distal to the bundle branch block can normalize the QRS complex in case of pure His bundle capture and absence of pre-excitation of adjacent myocardium. More recent studies demonstrating ventricular resynchronization through His bundle pacing in CRT candidates reinforced this idea $^{16}$.

\section{Electro-anatomic insights in the pathobiology of electrical dyssynchrony}

After the first crude mapping studies in LBBB and right ventricular (RV) paced patients by Vasallo et al. in the $1970 \mathrm{~s}^{17}$, detailed mapping studies were performed by Auricchio and associates $^{18,19}$. These studies showed that LBBB was characterized by a slow impulse conduction originating from the RV free wall gradually propagating to the LV lateral wall ${ }^{19}$. Interestingly, a large variation in transseptal conduction time (TST) was present in patients 
referred for CRT. While a few patients almost had simultaneous activation at the RV and LV side of the septum (TST near 0), most of the patients with LBBB showed TSTs of $>30 \mathrm{~ms}$, even up to $80 \mathrm{~ms}^{20}$. Long TSTs were also found in canine LBBB hearts, especially in canines with LBBB and tachypacing-induced heart failure $(\mathrm{HF})^{21}$. Slow transseptal conduction is also evident from epicardial contact maps in ventricular paced canine hearts ${ }^{7}$ and from non-invasive electrocardiographic imaging (ECGI) maps in LBBB patients (Figure 1) 22 . The slow conduction across the septum may be explained by its transverse conduction perpendicular to the fiber orientation. However, this is also the case in conduction from endocardium to epicardium of the LV free wall and this conduction is still faster than across the septum ${ }^{10}$. Apparently, the septum has specific structural abnormalities that slow down conduction, especially after remodelling due to HF. The slow septal conduction reflects an electrical separation between activation of the RV and LV, which is proposedly responsible for QRS notching on the electrocardiogram (ECG) characteristic for $\mathrm{LBBB}^{23}$.

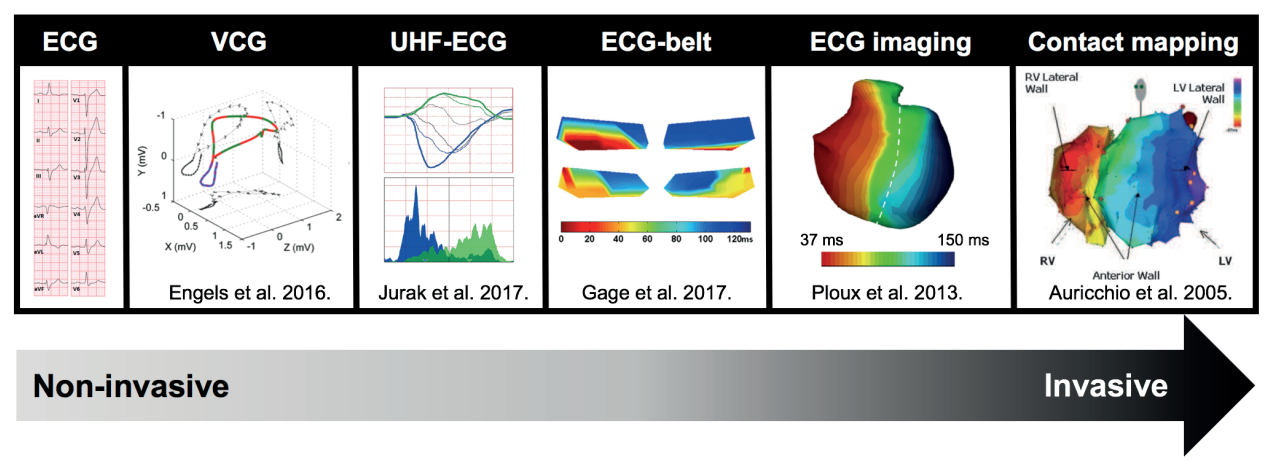

FIGURE 1. Overview of electrical dyssynchrony metric approaches. In the ECG imaging and contact mapping panels colour differences within the ventricles represent intraventricular dyssynchrony, whereas the mean difference in colour between the ventricles reflects interventricular dyssynchrony. ECG = electrocardiogram, VCG = vectorcardiography, $\mathrm{UHF}=$ ultra-high frequency.

\section{Non-invasive assessment of electrical dyssynchrony}

Much information about ventricular conduction abnormalities has been obtained using the 12-lead ECG. QRS duration $\geq 150 \mathrm{~ms}$ and a LBBB morphology on the ECG indicate a class $1 \mathrm{~A}$ recommendation for CRT implantation ${ }^{24}$. However, QRS duration cannot distinguish between right- or left-sided conduction abnormalities and between inter- and intraventricular dyssynchrony. While QRS morphology provides more information, it is prone to subjective interpretation and there are multiple definitions for specific conduction disturbances such 
as LBBB. For example, in a small retrospective study of different LBBB criteria in patients receiving $\mathrm{CRT}$, a 23\% disagreement in LBBB classification was found between the European and American guidelines ${ }^{25}$.

During recent years, multiple non-invasive techniques have been developed that allow more precise analysis of electrical dyssynchrony by incorporation of spatial or temporal information. First of all, there are techniques closely related to the conventional 12-lead ECG, such as vectorcardiography (VCG) and ultra-high frequency ECG that can be easily implemented in clinical practice ${ }^{26,27}$. A more complicated technology, ECGI, reconstructs the electro-anatomic activation of the epicardium based on body surface potential measurements using 200 electrodes around the chest and a patient-specific heart-torso geometry ${ }^{28}$. ECGI allows assessment of both inter- and intraventricular dyssynchrony ${ }^{22}$. Interestingly, it was shown that a parameter describing interventricular dyssynchrony correlates better with CRT response than parameters of intraventricular dyssynchrony; an observation that was subsequently supported by computer simulations 22,29 .

An intermediate approach between the conventional 12-lead ECG and the extensive ECGI is the ECG-belt, where 53 chest electrodes are used. The standard deviation of all ECGbelt-derived activation times (SDAT) has been proposed as a novel electrical dyssynchrony metric reflecting electrical heterogeneity. In 66 CRT recipients SDAT, but not QRS duration or morphology, was associated with LV end-systolic volume (LVESV) reduction and increase of LV function upon starting $\mathrm{CRT}^{30}$. An overview of the aforementioned electrical dyssynchrony assessment techniques is provided in Figure 1.

\section{Mechanics of the dyssynchronous ventricle}

A close relationship exists between excitation and contraction in both the normal ${ }^{31,32}$ and failing heart ${ }^{33,34}$. The latter was most clearly demonstrated by the use of $3 \mathrm{D}$ electromechanical mapping, which allows measuring strains and electrical activation at exactly the same position ${ }^{33}$.

This coupling between excitation and contraction makes it understandable that dyssynchronous electrical activation leads to dyssynchronous ventricular contraction. These contraction abnormalities are complex, as illustrated in Figure 2. Early shortening in early-activated regions is followed by a systolic rebound stretch, sometimes showing a bi- or triphasic pattern. In contrast, late-activated regions are subjected to early-systolic prestretch, followed by augmented systolic shortening that continues into diastole. The most likely physiological explanation for these patterns is that early contraction stretches the not-yet depolarized late-activated regions and that this prestretch activates the local Frank-Starling mechanism 
and subsequently creates a supranormal contraction in late-activated regions. Possibly the best support for this idea are results from simulations in the CircAdapt computer program. In this model, the ventricles are represented by three wall segments (RV, septum and LV free wall), which contain the property of length-dependent activation. Simply delaying LV free wall contraction results in strain patterns in septum and LV free wall that are close to those observed in LBBB patients (Figure 2A) ${ }^{35}$.

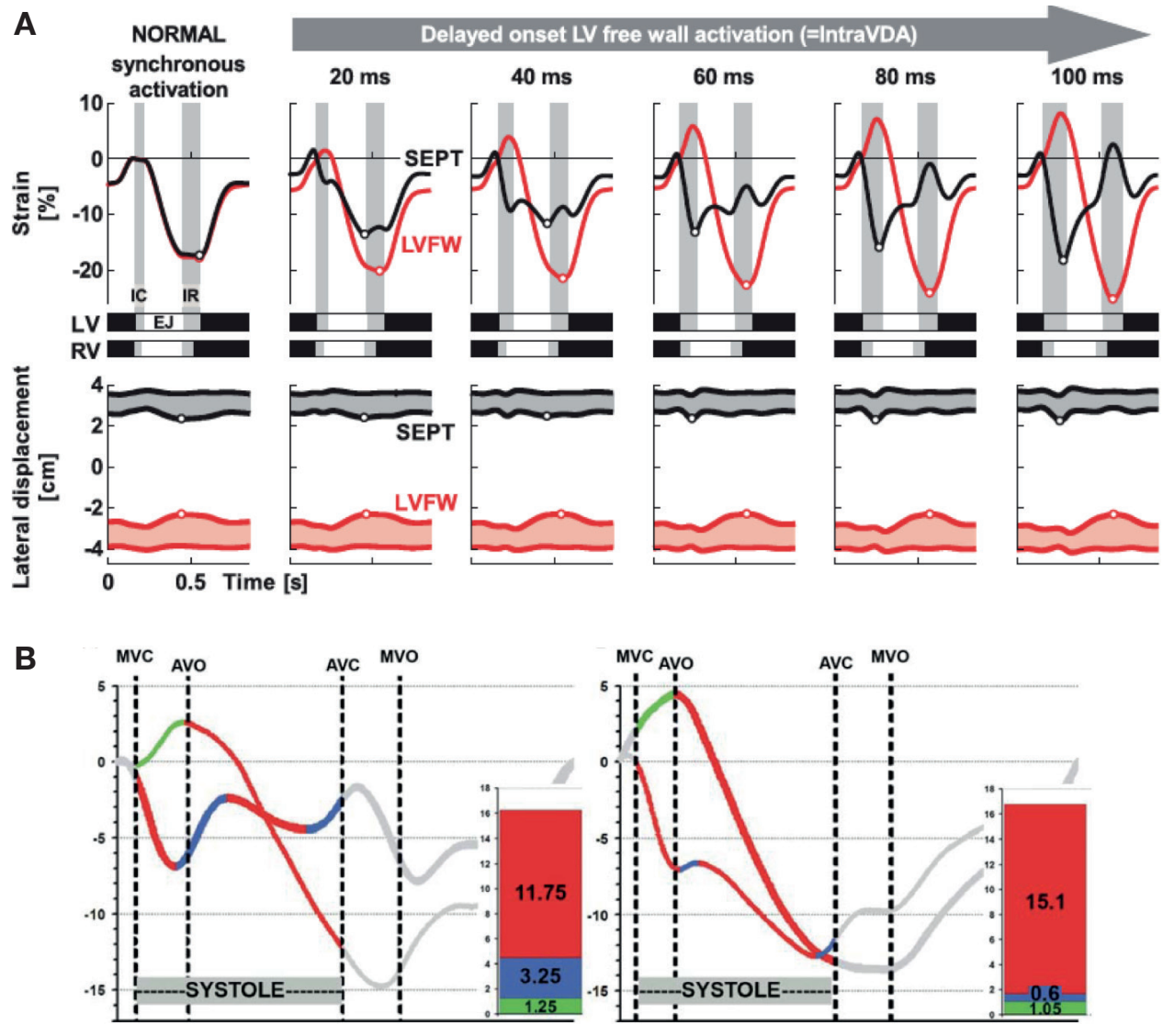

FIGURE 2. A. Effect of dyssynchronous ventricular activation on mechanical dyssynchrony indices in the CircAdapt model. Computer simulations were performed to predict LV septal and lateral wall strain curves after inducing LVFW delays in increasing severity. B. Strain patterns measured in a patient before (left) and during CRT (right). Red lines indicate systolic shortening, green early systolic prestretch and blue septal rebound stretch. The bar graphs indicate that CRT reduces septal rebound stretch and increases systolic shortening of the septum (thick lines) and LVFW (thin lines) combined. AVC = aortic valve closing, AVO = aortic valve opening, CRT = cardiac resynchronization therapy, $\mathrm{EJ}=$ ejection, IntraVDA = intraventricular delayed activation, IC = isovolumic contraction, IR = isovolumic relaxation, $\mathrm{LV}=$ left ventricle, $\mathrm{LVFW}=$ left ventricular free wall, $\mathrm{MVC}=$ mitral valve closing, $\mathrm{MVO}=$ mitral valve opening, $\mathrm{RV}=$ right ventricle, SEPT $=$ septal. Adapted from ${ }^{140}(A)$ and ${ }^{37}(B)$. 


\section{Assessment of mechanical dyssynchrony}

The analysis of mechanical dyssynchrony has developed rapidly over the last two decades. Initially tissue Doppler imaging was used ${ }^{36}$, while more recently, techniques to measure strains (length changes) emerged, such as speckle tracking echocardiography ${ }^{37}, 38$, cardiac magnetic resonance imaging $(\mathrm{CMR})$ tagging ${ }^{31,39}$ and more recently feature tracking $\mathrm{CMR}^{40}$ and $\mathrm{CT}$ SQUEEZ ${ }^{41}$. However, still none of the guidelines recommend these techniques for selection of CRT patients. This is explained by the results of randomized clinical studies investigating the use of mechanical dyssynchrony for improving the selection of patients for CRT. Mechanical dyssynchrony had no additional predictive power on top of ECG parameters in cohorts of patients with a wide QRS complex ${ }^{42,43}$, whereas neutral or even negative results were found when mechanical dyssynchrony was used as the only selection criterion in patients with a narrow QRS complex ${ }^{44,45}$.

In part, these poor results can be explained by limitations of tissue Doppler imaging ${ }^{46}$ and the strong operator dependency of acquisition and analysis of echocardiographic images. On the other hand, considerable differences in time to peak shortening may not only be caused by electrical dyssynchrony but also by regions with low contractility and scar ${ }^{47}$. Furthermore, peak shortening delay has at best a semi-quantitative relation with true dyssynchrony (Figure 2A). On the other hand, several studies showed the strength of relatively simple measures of dyssynchrony, such as apical rocking and septal flash do improve prediction of CRT response ${ }^{48-50}$. Further improvements were made by using the analysis of strain patterns ${ }^{51}$, resulting in parameters such as CURE ${ }^{52}$, septal systolic rebound stretch ${ }^{37,53}$, and systolic stretch index ${ }^{47}$.

\section{Functional consequences of mechanical dyssynchrony}

A direct consequence of ventricular dyssynchrony is the reduction in LV function. The goldstandard way to assess LV function is to determine pressure-volume (PV) loops. Immediately after the onset of dyssynchrony, the PV loop shows a rightward shift, indicating that the LV needs to operate at a larger volume in order to generate the same pressure; furthermore, stroke volume decreases ${ }^{54}$. The reverse process has been observed upon starting CRT in patients ${ }^{55}$. This poorer pump function is largely caused by the reduced systolic shortening in early-activated regions, which is hardly compensated by an increase in shortening in lateactivated regions, at least during the ejection phase. As a consequence, in LBBB hearts septal rebound stretch is a good predictor of CRT response ${ }^{37}$.

Such septal stretch during systole implies that this region dissipates energy that was generated in opposing regions ${ }^{56,57}$. In this respect Russell et al. introduced the term "wasted work" 57,58 . Using an elegant method, these investigators were able to show that the ratio of wasted 
and positive work predicts CRT response ${ }^{57}$. Importantly, the same research group recently showed that increasing afterload increases the amount of wasted work and disproportionally reduces global longitudinal strain and LV ejection fraction (LVEF) ${ }^{59}$. This finding suggests that the dyssynchronous heart is more sensitive to additional increases in cardiac workload than synchronous hearts and that the afterload should be taken into account when quantifying mechanical dyssynchrony.

The acute hemodynamic benefit of CRT can be understood from its correction of electromechanical dyssynchrony. Certainly in the heart with LBBB, CRT achieves a great deal of normalization of strain patterns and almost vanishing of septal rebound stretch ${ }^{37}$ and wasted work ${ }^{57}$.

A further consequence of the wasted work in the dyssynchronous heart is that the efficiency of conversion of metabolic energy (myocardial $\mathrm{O}_{2}$ consumption) to mechanical energy (stroke work) at the level of the entire LV can be up to 30\% lower during LBBB or RV pacing as compared to normal activation ${ }^{60}$. Conversely, CRT increases ventricular efficiency, which translates into a lower myocardial perfusion requirement for the same amount of pump work performed $^{61-63}$ and into a larger perfusion reserve ${ }^{64}$. Because many CRT patients may also have compromised coronary perfusion, CRT may also lower the risk or amount of underperfusion. This all may explain why wasted work ${ }^{65}$ and the work ratio between the septum and LV free wall ${ }^{66}$ are good predictors of CRT response.

A third consequence of dyssynchrony is the considerable redistribution of myocardial work within the ventricles. Because systolic shortening (so shortening against a pressure) in the septum is approximately zero, external work is also zero. In contrast, systolic shortening and external work are supranormal in the LV lateral wall (Figure 2). Accordingly, also myocardial blood flow is redistributed, both in experimental LBBB models ${ }^{67,68}$ and in patients ${ }^{62}$. The fact that this redistribution is reversed by CRT, concurrent with the homogenization of local strains and work ${ }^{68}$ indicates that workload drives flow and not the other way around. Earlier work of Amitzur supports this view by showing that administration of adenosine in RV paced hearts changes local blood flow but not contraction patterns ${ }^{69}$.

Figure 3 summarizes how the various acute consequences of dyssynchronous electrical activation, mentioned above, lead to worsening cardiac function and ventricular dilatation. In clinical terms, the diminishment of systolic function is evidenced by a lower LVEF and lower strains, worse diastolic function by reduced diastolic filling times and the dilation is shown by a larger LV end-diastolic volume and a rightward shift of the PV loop in conductance catheter measurements ${ }^{55}$. 


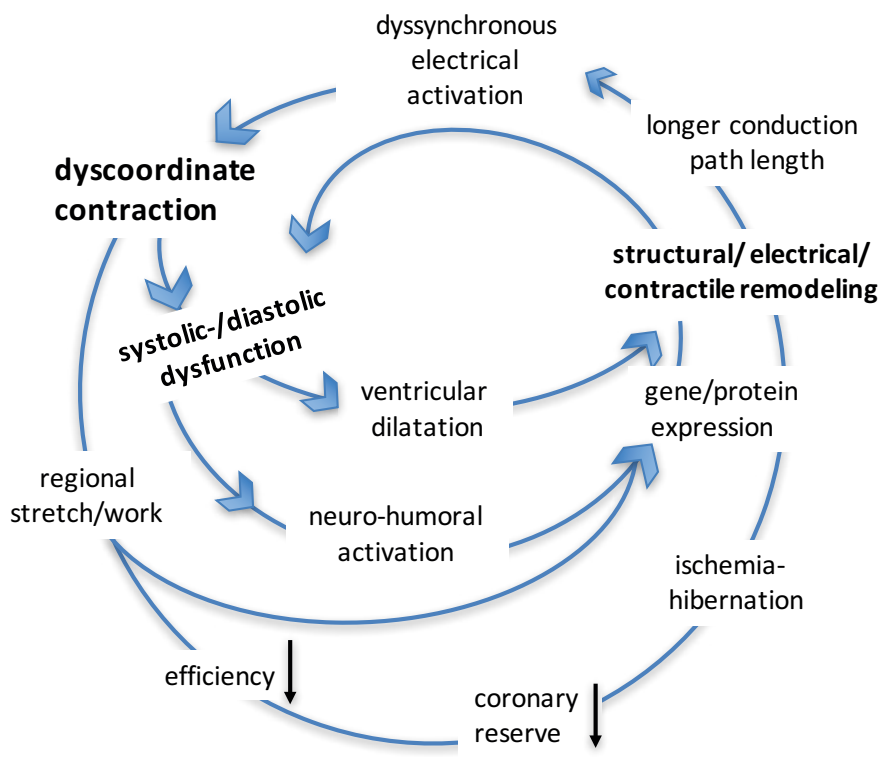

FIGURE 3. Schematic representation of maladaptive processes following the onset of dyssynchronous activation (see text for details).

\section{Remodelling: from myocardium to molecule}

Figure 3 also depicts that "dyssynchronous HF" leads to unique and extensive gene and protein expression patterns that can be linked to structural as well as electrophysiological and contractile changes ${ }^{70-72}$. These "remodelling processes" are driven by neurohumoral factors, like adrenergic stimulation and activation of the renin-angiotensin-aldosterone system (RAAS), and by the mechanical load on the heart itself ${ }^{73}$. Although the exact mechanisms for transmitting mechanical load to the myocardial cells are not clear $^{74}$, stretch is a well-known trigger for changing cellular and organ function, both in vitro and in vivo ${ }^{54,75-77}$. Typically, in LBBB, the early systolic prestretch and augmented external work in late-activated regions seem logical explanations for the frequently more pronounced remodelling processes in these regions.

Because in-depth understanding of (reverse) remodelling processes requires invasive procedures, like biopsies, most knowledge in the field of dyssynchrony and resynchronization is derived from various animal models of dyssynchrony ${ }^{78}$. The most straightforward way is to induce dyssynchrony by RV pacing or by LBBB, induced using radio frequency ablation while maintaining a normal heart rate. However, since such dyssynchrony reduces pump 
function but does not lead to HF within a period of half a year ${ }^{67}$, several groups have added an increase in heart rate, ranging from 120 to 220 bpm for a period of 3-4 weeks to 24 months ${ }^{79}$, 80. In all these models, resynchronization has been employed using biventricular pacing. Importantly, studies with tachypacing dyssynchronous HF also use tachy-biventricular pacing to resynchronize, which will be referred to as tachy-CRT. Tachy-CRT hardly improves functional parameters like LVEF, but reveals many changes at the cellular and molecular level presumably as a consequence of the better coordination of contraction ${ }^{81,82}$.

\section{Structural remodelling}

In patients with dyssynchronous HF, CRT induces reverse remodelling with a reduction in LVESV $^{83,84}$ and reduced fibrosis ${ }^{85,86}$. CRT responders (defined by LVESV reduction) were also shown to have a decreased LV mass and regional wall thickness, with improved LV geometry, as determined by the sphericity index ${ }^{87,88}$. An interesting observation is that polymorphisms in the mineralocorticoid receptor gene proved an important determinant for reverse remodelling in CRT patients ${ }^{89,90}$. This supports the view that the RAAS is strongly involved in the remodelling process in dyssynchronous failing hearts. These clinical studies suggest that the capability to reverse the remodelling is an important determinant of long-term CRT response. It seems reasonable to suggest that the overall decrease in LV wall mass and the reduced fibrosis upon CRT are mediated by the improved LV systolic function. A reduction in LV cavity volume decreases wall stress and, together with the increased LV function, potentially lowers neurohumoral activation. The reduction in workload in the late-activated LV free wall may be responsible for the excess reduction in LV free wall mass.

Animal models of LBBB with natural heart rate show ventricular dilatation in combination with asymmetric hypertrophy, the most pronounced hypertrophy occurring at sites of late activation ${ }^{54}$, 68. The presence of this asymmetric hypertrophy (measured both by echocardiography and post-mortem histology ${ }^{54,91}$ ) is accompanied by local molecular changes such as decreased miR133a expression and overexpression of connective tissue growth factor (CTGF) selectively in the LV lateral wall ${ }^{91}$. These local changes and their reversal upon applying CRT ${ }^{68,91}$ are a strong indication of the importance of local mechanics for remodelling, because all regions of the dyssynchronous heart are subjected to the same neurohumoral stimulation. While the increase in LV cavity volume after onset of LBBB may directly follow the loss in LV systolic function (Figure 3), further LV dilatation may be mediated by changes in the extracellular matrix $(E C M)$ properties and matrix metalloproteases (MMPs). This fits with the finding of ECM-remodelling and increased MMP activity in the LV free wall of RV-paced dogs ${ }^{92}$ and in dogs with tachypacing-induced dyssynchronous $\mathrm{HF}^{80,93}$. The RV-pacing study also showed ECM accumulation (fibrosis) in the late-activated LV free wall92. By contrast, in dog-models of dyssynchrony without significant cardiac dysfunction (LBBB or LV pacing), myocardial 
collagen content was not affected ${ }^{54,91,94}$, although this did occur in a study of tachypacinginduced dyssynchronous HF80. In patients, a modest reduction in fibrosis was observed after $\mathrm{CRT}^{85,86}$, which may be explained by a lower neurohumoral activation. Also, expression of paracrine factors contributing to fibrosis or hypertrophy, including osteopontin, transforming growth factor beta (TGFB), CTGF and B-type natriuretic peptide (BNP), is increased during dyssynchrony and (partly) normalized by $\mathrm{CRT}^{91,93,95}$.

Besides cardiomyocyte hypertrophy and ECM accumulation, apoptosis contributes to the cardiac remodelling process in dyssynchronous HF. An overall increase in various proapoptotic factors (such as caspases) has been shown in dyssynchronous HF ${ }^{96,97}$. This proapoptotic state is ameliorated by CRT and the regional variance in the expression of several stress-kinases is reduced ${ }^{96}$. The pro-apoptotic factor tumour necrosis factor alfa (TNFa) is increased in animal models of dyssynchrony ${ }^{96,98}$ and in patients eligible for $\mathrm{CRT}^{86}$. In the animal model, the most pronounced increase in TNFa levels occurs in the LV free wall, with tachyCRT partially normalizing TNFa expression ${ }^{96}$. In accordance with this, also patients show reduced TNFa levels following CRT ${ }^{85,86}$. Moreover, DNA fragmentation, a marker of apoptosis, is increased in dyssynchronous HF and reduced by CRT $85,96,97$. Interestingly, increased DNA fragmentation occurs in both the septum and the LV free wall and tachy-CRT is capable of reducing the occurrence of this process in both locations ${ }^{96}$.

\section{Electrical remodelling}

Electrical remodelling in dyssynchronous and resynchronized hearts may affect the sequence of both depolarization and repolarization.

Changes in the depolarization sequence have been observed from the broadening of the QRS complex during longer-lasting dyssynchrony in animal models ${ }^{54,67}$. Similarly, longerlasting CRT has been reported to decrease QRS duration (both the paced and the non-paced QRS complex) in patients ${ }^{99}$. Beside changes in the gross anatomy (changes in LV mass and diameter and degree of fibrosis) discussed above, also changes in ion channels may play a role. Figure $4 \mathrm{~A}$ schematically illustrates the contribution of the various ion channels to the ventricular action potential.

Cx43 is the main gap junction protein responsible for sodium influx during phase 0 of the action potential and subsequently conduction velocity. Increased lateralization of Cx43 in the LV is associated with remodelling processes after tachypacing-induced HF and after myocardial infarction (Figure 5) ${ }^{100,101}$. Interestingly, electrical dyssynchrony by itself already influences Cx43 localization. In the lateral segments of the canine LBBB heart, a reduced endocardial conduction velocity was observed in combination with lateralization of Cx4394. Lateralization of Cx43 may lead to a more zig-zag conduction pattern ${ }^{102}$, and consequently slower conduction, 
heterogeneity of refractoriness, and increased risk of reentry circuits. In addition, in canine hearts with tachypacing-induced HF both lateralization and decreased expression of Cx43 were associated with slowing of His-Purkinje conduction (Figure 4B) ${ }^{103}$, indicating that remodelling processes secondary to HF alone already prolong QRS duration.

Another factor contributing to conduction and cardiac excitation is the fast-inward voltagedependent sodium channel $\left(\mathrm{I}_{\mathrm{Na}}\right)$, but its role in dyssynchrony is equivocal. $\mathrm{I}_{\mathrm{Na}}$ density was decreased in explanted human hearts with $\mathrm{HF}^{104}$, but contradictory results have been reported for canine hearts with tachypacing-induced $\mathrm{HF}^{104,105}$.

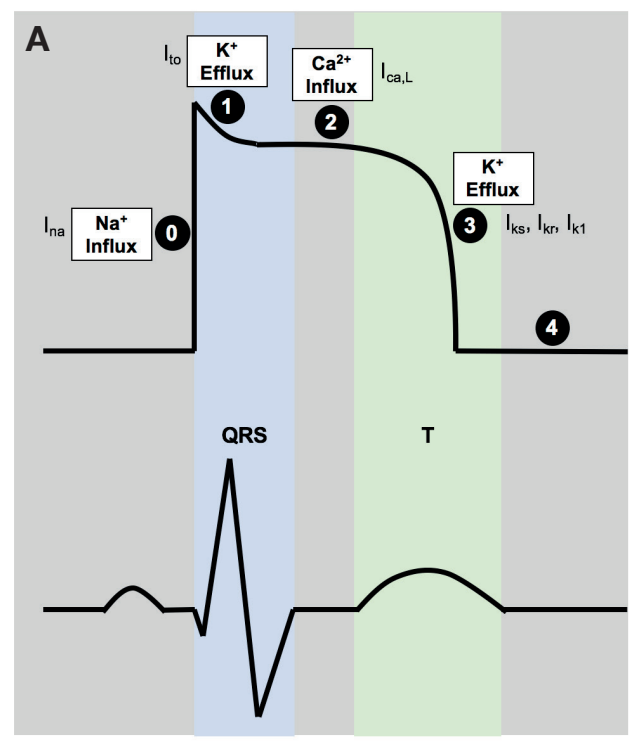

B
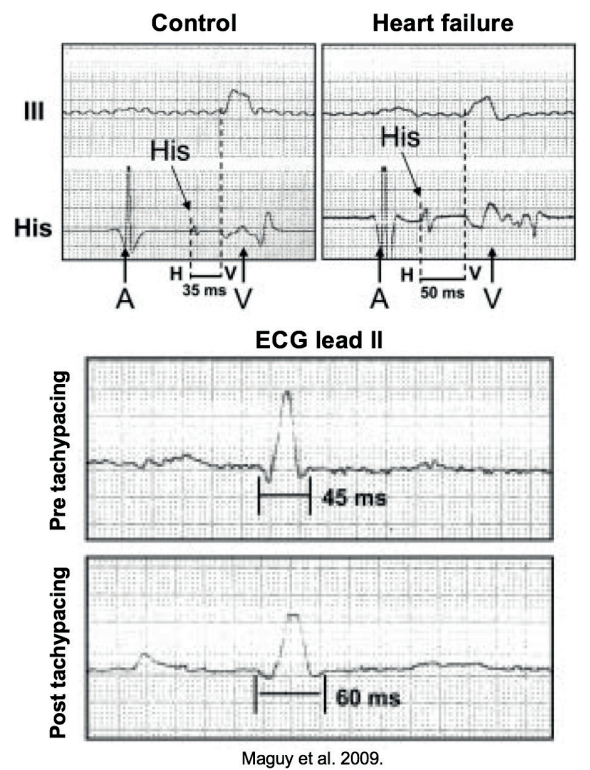

FIGURE 4. A. Schematic overview of the five phases of the action potential, ECG, and ion channels involved. The action potential consists of: phase 0 (upstroke), phase 1 (fast early repolarization), phase 2 (plateau), phase 3 (repolarization), and phase 4 (resting membrane potential). B. ECG and His electrograms demonstrating an increase in HV-interval and QRS duration after tachypacing-induced heart failure in canine hearts (adapted from ${ }^{103}$ ). $\mathrm{ECG}=$ electrocardiogram.

While changes in the QRS complex reflect changes in depolarization, $T$ wave changes reflect altered repolarization. In this respect, "cardiac memory" is of interest. Cardiac memory refers to persistent $T$ wave changes on the ECG during restoration of normal ventricular activation sequence after a period of abnormal ventricular activation. These persistent $T$ wave abnormalities indicate that the sequence of repolarization adapts to a new activation sequence $^{106}$. This phenomenon has been studied in animals and humans ${ }^{107,}{ }^{108}$. In patients 
with RV pacing, changes in T wave amplitude and duration were already present within one day of pacing and became persistent after one week ${ }^{109}$. Similar cardiac memory phenomena developed between one day and two weeks of CRT ${ }^{110}$. Interestingly, animal studies showed that cardiac memory could be suppressed by mechanical unloading of the heart ${ }^{107}$. At the molecular level, involvement of the L-type calcium channel'11 ${ }^{11}$ and the cyclic adenosine monophosphate response element binding protein, a factor heavily involved in regulation of gene expression, was shown ${ }^{112}$. These results strongly argue that the ventricular repolarization changes underlying cardiac memory are related to the mechanical consequences of the activation sequence ("mechano-electrical feedback").

While the T wave changes in the setting of cardiac memory are indicative of changes in action potential duration (APD), such APD changes have indeed been observed in normal animal hearts during longer-lasting periods of ventricular pacing ${ }^{113}$. These studies showed APD lengthening in early-activated regions and gradual APD shortening towards later-activated regions. However, in the very latest-activated regions the APD lengthened in the dog heart ${ }^{114}$, whereas it was shortened in rabbit hearts ${ }^{113}$.

Commonly, the upright "concordant" $T$ wave in the normal ECG is explained by late-activated regions having a shorter APD than early-activated regions ${ }^{115}$. While cardiac memory indicates some potential of the ventricles to adapt APD to activation sequence, the usually discordant $T$ wave in hearts with RV pacing and LBBB suggests that this potential to adjust APD is insufficient in case of severe dyssynchrony. However, studies in patients without HF show that during longer-lasting dyssynchrony (RV pacing and LBBB), the amplitude of the $T$ wave decreases over time ${ }^{108,116,117}$, suggesting some adaptation to the abnormal conduction. However, a recent electrical mapping study in HF patients showed that, regardless of QRS duration and morphology, the sequence of repolarization largely follows the sequence of activation, pointing towards reduced "capacity" of failing myocardium to adjust APD to abnormal activation sequence ${ }^{118}$.

Concordant with the experimentally observed role of the L-type calcium channel in cardiac memory, data from tachypaced dyssynchronous dog hearts show differential expression and current flow of this calcium channel, with lower values in the LV lateral than in the anterior wall81. However, while larger L-type calcium currents are expected to prolong APD, in the same study APD was shorter in myocytes from earlier-activated LV anterior wall regions ${ }^{81}$. The investigators explain this paradox by referring to the slower decay in the current in the LV lateral wall. In addition, the severe, but uniform reduction in various potassium channels (important during phase 3 and 4 of the action potential, Figure 4A), may change the influence of the various ion channels during the repolarization phase ${ }^{81}$. 
Computer model studies investigated how to reconcile the aforementioned mechano-electrical feedback, observed in cardiac memory studies, and the electrical and contractile remodelling in dyssynchronous hearts. It was assumed that mechano-sensing aims to maintain a constant mechanical function (like strain), and that adjustments occur through varying activity of the L-type calcium current ${ }^{81}$. According to the computer simulation, a reduction in local mechanics in early-activated regions was followed by increased activity of the L-type calcium channel ${ }^{119}$. During small time differences in activation (e.g. sinus rhythm), this adaptation process was able to lead to the well-known concordant $T$ wave ${ }^{119}$. In the dyssynchronous ventricle, $T$ waves only became less discordant over time ${ }^{120}$, much like what has been observed in patients (see above).

Clearly, our understanding of electrical remodelling processes influencing ventricular repolarization in the dyssynchronous heart is incomplete. Beside factors discussed above, also the poor understanding of electrotonic influences on repolarization in vivo and of the relation between APD heterogeneity and T wave morphology should be recognized ${ }^{115,118}$.

\section{Contractile remodelling}

Dyssynchrony results in acute negative effects on LV contractile function in animals ${ }^{68}$ and patients ${ }^{121}$. Moreover, a study in patients with normal LVEF showed that RV pacing immediately reduces LVEF by $\sim 6 \%$, followed by a further $\sim 8 \%$ during the subsequent week of RV pacing. Full recovery of LVEF after termination of pacing took 2 days, further indicating some kind of contractile remodelling ${ }^{122}$. Similarly, CRT reverses some of the contractile defects beat-tobeat $^{123,124}$ and continues to do so over years ${ }^{68,125}$.

While the acute hemodynamic effects can be explained by changes in mechanical coordination, as explained above, the chronic effects seem mediated by subcellular and molecular changes ${ }^{4}$. In this regards calcium probably plays a dual role, also being involved in electrical remodelling. As mentioned above, in the tachypacing model LBBB led to a significant and regionally different reduction in the L-type calcium current and this disparity was resolved by tachypacing $C R T^{81}$. L-type calcium current densities were also reduced in ventricular myocytes from a minipig model of RV pacing, compared to controls ${ }^{126}$.

Also, other proteins involved in calcium handling may play a role in contractile remodelling. Patients with ischemic or idiopathic dilated cardiomyopathy show reduced sarcoplasmatic reticulum (SR) $\mathrm{Ca}^{2+}$ ATP-ase (SERCA) activity compared to controls ${ }^{127}$. Lower SERCA expression was found in patients who are eligible for $\mathrm{CRT}^{95}$ and CRT increases SERCA expression ${ }^{128,129}$. Vanderheyden et al. showed that the increase in SERCA only occurs in CRT responders ${ }^{95}$. Several ${ }^{128,129}$, albeit not all studies report an increase in phospholamban (PLN) levels after $\mathrm{CRT}^{95}$. 
These clinical data fit with observations of reduced SR calcium uptake in a porcine model of RV pacing ${ }^{98}$ and downregulation of SERCA in tachypaced ${ }^{81}$ and non-tachypaced ${ }^{130}$ dog hearts. In one study, the change occurred in the LV free wall but not in the interventricular septum ${ }^{130}$. In the LV free wall of tachypaced dyssynchronous HF a reduction in SERCA expression was selectively found in the endocardium, leading to a steep transmural expression gradient ${ }^{79}$. After tachy-CRT, SERCA was no longer significantly downregulated in either early- or late-activated myocardium ${ }^{81}$. Slightly smaller, but still significant reductions were observed for PLN in both tachypaced and non-tachypaced canine models of dyssynchrony ${ }^{81,130}$, but not in a porcine model ${ }^{98}$.

An upregulation of the sodium-calcium exchanger (NCX) was found in animal models of (tachy)dyssynchrony ${ }^{81,98}$ and a similar trend could be observed after CRT in patients ${ }^{129}$.

These molecular changes may relate to subcellular changes. In patients, abnormalities in T-tubular structure have been linked to impaired contractility ${ }^{131}$. In the LV lateral wall of the tachypacing LBBB model, regression of the T-tubular system has been reported, which was partially reversible upon tachy-CRT ${ }^{132,133}$.

An important role in the contractile remodelling processes may also be reserved for the autonomic nervous system. The response to $\beta$-adrenergic stimulation is diminished in patients with $\mathrm{HF}^{134}$. CRT treatment recovers the $\beta$-adrenergic response ${ }^{135}$.

On the cellular level, the contractile response to $\beta$-adrenergic stimulation (increase in calcium transient, cellular shortening) is reduced in $\mathrm{HF}^{136}$, which may be (partially) explained by downregulation of the $\beta 1$ and $\beta 2$ adrenergic receptors ${ }^{137,138}$. In tachypaced dyssynchronous $\mathrm{HF}$, tachy-CRT normalizes the response in cells from both anterior and lateral LV wall ${ }^{137}$ ${ }^{138}$. Interestingly, most studies, in both patients ${ }^{129}$ and animals ${ }^{137,}{ }^{138}$, demonstrate that CRT upregulates the $\beta 1$-, but not the $\beta 2$-receptor. In addition, a unique feature of $\beta 2$-receptor signaling is observed during dyssynchrony, namely an increased G-protein alpha-I (Ga) coupling, leading to reduced adenylyl cyclase activity and thus lowering cyclic AMP (CAMP) production. This contributes to decreased contractile function. Upon resynchronization, the $\beta 2$ receptor becomes uncoupled from these $\mathrm{Ga}_{\mathrm{i}}$ proteins and restores $\mathrm{Ga}_{\mathrm{s}}$ signaling ${ }^{137}$. This this mechanism may also be active in CRT responders ${ }^{137}$.

Also at the level of the myofibrils some remodelling may be present, for instance in myosin heavy chain a (MHCa). In patients, dyssynchronous $\mathrm{HF}$ is associated with reduced expression of $\mathrm{MHCa}$ and this reduction seems reversed by $\mathrm{CRT}^{95}$, 128. The CRT-induced MHCa increase correlated with the reduction in cavity dimensions ${ }^{128}$. 
Studies in skinned muscle fibers from animals with tachypacing-induced HF showed yet another mechanism of contractile modulation: calcium sensitivity of the myofilaments. This sensitivity was shown to be reduced in dyssynchronous HF due to glycogen synthase kinase (GSK)-3 $\beta$-dependent phosphorylation of contractile proteins. Tachy-CRT restored calcium sensitivity through increased GSK3- $\beta$ activity ${ }^{139}$.

Summarizing, a large range of processes seem responsible for the contractile remodelling processes in dyssynchrony and its reversal upon CRT, which are depicted in Figure 5.
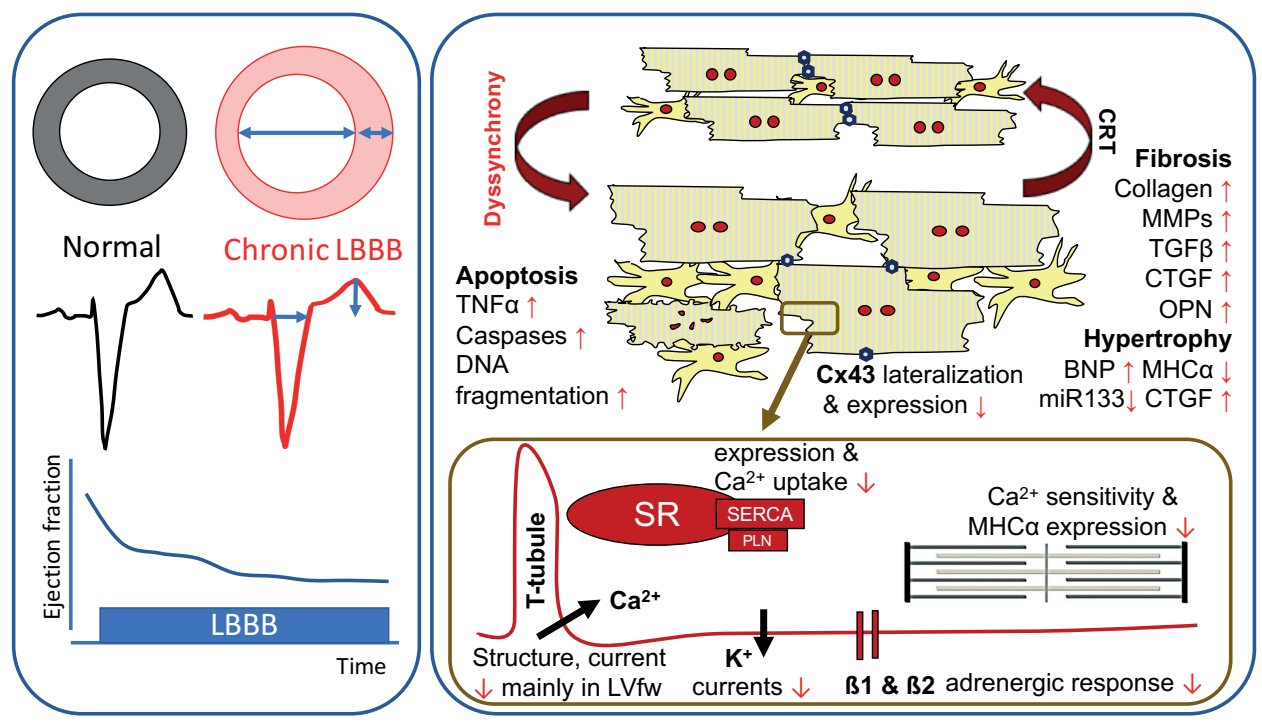

FIGURE 5. Processes contributing to the structural, electrical, and contractile remodelling in the dyssynchronous heart as seen on functional measurements (left) and on a cellular and molecular level (right). Red colour indicates the situation during dyssynchrony. Dyssynchrony causes asymmetric, eccentric hypertrophy and (in the failing heart) fibrosis as well as apoptosis. Some of the molecular factors are mentioned. Similarly, some of the processes involved in altered excitation-contraction coupling are displayed in the inset, illustrating a part of the plasmalemma, T-tubule and sarcoplasmic reticulum. See text for further details. BNP = B-type natriuretic peptide, CRT = cardiac resynchronization therapy, CTGF = connective tissue growth factor, Cx43 = connexin 43, LBBB = left bundle branch block, LVfw = LV free wall, MHCa = myosin heavy chain a, MMPs = matrix metalloproteases, OPN = osteopontin, $\mathrm{PLN}=$ phospholamban, $\mathrm{SR}=$ sarcoplasmic reticulum, SERCA $=\mathrm{SR} \mathrm{Ca}^{2+}$ ATP-ase, TGF $\beta=$ transforming growth factor $\beta$, TNFa = tumour necrosis factor $a$. 


\section{Conclusion}

This review illustrates that conduction abnormalities (such as LBBB) lead to extensive immediate and long-term changes in the heart and reversal of functions by CRT. The long-term adaptations of the myocardium can be characterized as "maladaptive" because many functions continue to decrease over time. This also explains why CRT, unlike most pharmacological treatments, continues to increase its therapeutic effect over time. 


\section{References}

1. Myerburg RJ, Gelband H, Nilsson K, Castellanos A, Morales AR, Bassett AL. The role of canine superficial ventricular muscle fibers in endocardial impulse distribution. Circ Res 1978; 42: 27-35.

2. Ono N, Yamaguchi T, Ishikawa H, Arakawa M, Takahashi N, Saikawa T, et al. Morphological varieties of the Purkinje fiber network in mammalian hearts, as revealed by light and electron microscopy. Arch Histol Cytol 2009; 72: 139-149.

3. Oosthoek PW, Viragh S, Lamers WH, Moorman AF. Immunohistochemical delineation of the conduction system. II: The atrioventricular node and Purkinje fibers. Circ Res 1993; 73: 482-491.

4. Strik M, Rademakers LM, van Deursen CJ, van Hunnik A, Kuiper M, Klersy C, et al. Endocardial left ventricular pacing improves cardiac resynchronization therapy in chronic asynchronous infarction and heart failure models. Circ Arrhythm Electrophysiol 2012; 5: 191-200.

5. Frazier OH, Macris MP, Duncan JM, Van Buren CT, Cooley DA. Cardiac transplantation in patients over 60 years of age. Ann Thorac Surg 1988; 45: 129-132.

6. Hyde ER, Behar JM, Claridge S, Jackson T, Lee AW, Remme EW, et al. Beneficial Effect on Cardiac Resynchronization From Left Ventricular Endocardial Pacing Is Mediated by Early Access to High Conduction Velocity Tissue: Electrophysiological Simulation Study. Circ Arrhythm Electrophysiol 2015; 8: 1164-1172.

7. Taccardi B, Punske BB, Macchi E, Macleod RS, Ershler PR. Epicardial and intramural excitation during ventricular pacing: effect of myocardial structure. Am J Physiol Heart Circ Physiol 2008; 294: H1753-1766.

8. Derval N, Jais P. Optimizing hemodynamics in cardiac resynchronization therapy by left ventricular pacing site: you find only what you are looking for. J Am Coll Cardiol 2010; 56: 782-783.

9. Durrer D, van Dam RT, Freud GE, Janse MJ, Meijler FL, Arzbaecher RC. Total excitation of the isolated human heart. Circulation 1970; 41: 899-912.

10. Spach MS, Miller WT, 3rd, Geselowitz DB, Barr RC, Kootsey JM, Johnson EA. The discontinuous nature of propagation in normal canine cardiac muscle. Evidence for recurrent discontinuities of intracellular resistance that affect the membrane currents. Circ Res 1981; 48: 39-54.

11. Demoulin JC, Kulbertus HE. Histopathological examination of concept of left hemiblock. Br Heart J 1972; 34: 807-814.

12. Eriksson $\mathrm{P}$, Hansson $\mathrm{PO}$, Eriksson $\mathrm{H}$, Dellborg M. Bundle-branch block in a general male population: the study of men born 1913. Circulation 1998; 98: 2494-2500.

13. El-Sherif N, Amay YLF, Schonfield C, Scherlag BJ, Rosen K, Lazzara R, et al. Normalization of bundle branch block patterns by distal His bundle pacing. Clinical and experimental evidence of longitudinal dissociation in the pathologic his bundle. Circulation 1978; 57: 473-483.

14. Narula OS, Javier RP, Samet P, Maramba LC. Significance of His and left bundle recordings from the left heart in man. Circulation 1970; 42: 385-396.

15. Narula OS. Longitudinal dissociation in the His bundle. Bundle branch block due to asynchronous conduction within the His bundle in man. Circulation 1977; 56: 996-1006. 
16. Aijjola OA, Upadhyay GA, Macias C, Shivkumar K, Tung R. Permanent His-bundle pacing for cardiac resynchronization therapy: Initial feasibility study in lieu of left ventricular lead. Heart Rhythm 2017; 14: 1353-1361.

17. Vassallo JA, Cassidy DM, Miller JM, Buxton AE, Marchlinski FE, Josephson ME. Left ventricular endocardial activation during right ventricular pacing: effect of underlying heart disease. J Am Coll Cardiol 1986; 7: 1228-1233.

18. Fantoni C, Kawabata M, Massaro R, Regoli F, Raffa S, Arora V, et al. Right and left ventricular activation sequence in patients with heart failure and right bundle branch block: a detailed analysis using threedimensional non-fluoroscopic electroanatomic mapping system. J Cardiovasc Electrophysiol 2005; 16: 112-119; discussion 120-121.

19. Auricchio A, Fantoni C, Regoli F, Carbucicchio C, Goette A, Geller C, et al. Characterization of left ventricular activation in patients with heart failure and left bundle-branch block. Circulation 2004;

109: 1133-1139.

20. Prinzen FW, Auricchio A. Is echocardiographic assessment of dyssynchrony useful to select candidates for cardiac resynchronization therapy? Echocardiography is not useful before cardiac resynchronization therapy if QRS duration is available. Circ Cardiovasc Imaging 2008; 1: 70-77; discussion 78.

21. Strik M, van Deursen CJ, van Middendorp LB, van Hunnik A, Kuiper M, Auricchio A, et al. Transseptal conduction as an important determinant for cardiac resynchronization therapy, as revealed by extensive electrical mapping in the dyssynchronous canine heart. Circ Arrhythm Electrophysiol 2013; 6: 682-689.

22. Ploux S, Lumens J, Whinnett Z, Montaudon M, Strom M, Ramanathan C, et al. Noninvasive electrocardiographic mapping to improve patient selection for cardiac resynchronization therapy: beyond QRS duration and left bundle branch block morphology. J Am Coll Cardiol 2013; 61: 24352443.

23. Strauss DG, Selvester RH, Wagner GS. Defining left bundle branch block in the era of cardiac resynchronization therapy. Am J Cardiol 2011; 107: 927-934.

24. Brignole M, Auricchio A, Baron-Esquivias G, Bordachar P, Boriani G, Breithardt OA, et al. 2013 ESC guidelines on cardiac pacing and cardiac resynchronization therapy: the task force on cardiac pacing and resynchronization therapy of the European Society of Cardiology (ESC). Developed in collaboration with the European Heart Rhythm Association (EHRA). Europace 2013; 15: 1070-1118.

25. van Deursen CJ, Blaauw Y, Witjens MI, Debie L, Wecke L, Crijns HJ, et al. The value of the 12-lead ECG for evaluation and optimization of cardiac resynchronization therapy in daily clinical practice. $J$ Electrocardiol 2014; 47: 202-211.

26. Jurak P, Halamek J, Meluzin J, Plesinger F, Postranecka T, Lipoldova J, et al. Ventricular dyssynchrony assessment using ultra-high frequency ECG technique. J Interv Card Electrophysiol 2017; 49: 245254.

27. van Deursen CJ, Vernooy K, Dudink E, Bergfeldt L, Crijns HJ, Prinzen FW, et al. Vectorcardiographic QRS area as a novel predictor of response to cardiac resynchronization therapy. $J$ Electrocardiol 2015; 48: 45-52. 
28. Ramanathan C, Ghanem RN, Jia P, Ryu K, Rudy Y. Noninvasive electrocardiographic imaging for cardiac electrophysiology and arrhythmia. Nat Med 2004; 10: 422-428.

29. Huntjens PR, Ploux S, Strik M, Walmsley J, Ritter P, Haissaguerre M, et al. Electrical substrates driving response to cardiac resynchronization therapy: a combined clinical - computational evaluation. Circ Arrhythm Electrophysiol 2018; 11: e005647.

30. Gage RM, Curtin AE, Burns KV, Ghosh S, Gillberg JM, Bank AJ. Changes in electrical dyssynchrony by body surface mapping predict left ventricular remodeling in patients with cardiac resynchronization therapy. Heart Rhythm 2017; 14: 392-399.

31. Wyman BT, Hunter WC, Prinzen FW, McVeigh ER. Mapping propagation of mechanical activation in the paced heart with MRI tagging. Am J Physiol 1999; 276: H881-H891.

32. Wyman BT, Hunter WC, Prinzen FW, Farris OP, McVeigh ER. Effects of single- and biventricular pacing on temporal and spatial dynamics of ventricular contraction. Am J Physiol 2002; 282: H372-H379.

33. Kroon W, Lumens J, Potse M, Suerder D, Klersy C, Regoli F, et al. In vivo electromechanical assessment of heart failure patients with prolonged QRS duration. Heart Rhythm 2015; 12: 12591267.

34. Mafi Rad M, van 't Sant J, Blaauw Y, Meijboom FJ, Doevendans PA, Cramer MJ, et al. Regional left ventricular electrical activation and contraction are closely related in candidates for cardiac resynchronization therapy. JACC Clin Electrophysiol 2017: 854-862.

35. Lumens J, Leenders GE, Cramer MJ, De Boeck BW, Doevendans PA, Prinzen FW, et al. Mechanistic evaluation of echocardiographic dyssynchrony indices: patient data combined with multiscale computer simulations. Circ Cardiovasc Imaging 2012; 5: 491-499.

36. Bleeker GB, Schalij MJ, Molhoek SG, Verwey HF, Holman ER, Boersma E, et al. Relationship between QRS duration and left ventricular dyssynchrony in patients with end-stage heart failure. $J$ Cardiovasc Electrophysiol 2004; 15: 544-549.

37. De Boeck BW, Teske AJ, Meine M, Leenders GE, Cramer MJ, Prinzen FW, et al. Septal rebound stretch reflects the functional substrate to cardiac resynchronization therapy and predicts volumetric and neurohormonal response. Eur J Heart Fail 2009; 11: 863-871.

38. Hara H, Oyenuga OA, Tanaka H, Adelstein EC, Onishi T, McNamara DM, et al. The relationship of QRS morphology and mechanical dyssynchrony to long-term outcome following cardiac resynchronization therapy. Eur Heart J 2012; 33: 2680-2691.

39. Bilchick KC, Dimaano V, Wu KC, Helm RH, Weiss RG, Lima JA, et al. Cardiac magnetic resonance assessment of dyssynchrony and myocardial scar predicts function class improvement following cardiac resynchronization therapy. JACC Cardiovasc Imaging 2008 1: 561-568.

40. Onishi T, Saha SK, Ludwig DR, Onishi T, Marek JJ, Cavalcante JL, et al. Feature tracking measurement of dyssynchrony from cardiovascular magnetic resonance cine acquisitions: comparison with echocardiographic speckle tracking. J Cardiovasc Magn Reson 2013; 15: 95.

41. Behar JM, Rajani R, Pourmorteza A, Preston R, Razeghi O, Niederer S, et al. Comprehensive use of cardiac computed tomography to guide left ventricular lead placement in cardiac resynchronization therapy. Heart Rhythm 2017; 14: 1364-1372. 
42. Chung ES, Leon AR, Tavazzi L, Sun JP, Nihoyannopoulos P, Merlino J, et al. Results of the Predictors of Response to CRT (PROSPECT) trial. Circulation 2008 117: 2608-2616.

43. Seo Y, Ito H, Nakatani S, Takami N, Naito S, Shiga T, et al. The role of echocardiography in predicting responders to cardiac resynchronization therapy: Results from the Japan Cardiac Resynchronization therapy registry Trial (J-CRT). Circ J 2011; 75: 1156-1163.

44. Ruschitzka M, Abraham WT, Singh JP, Bax JJ, Borer JS, Brugada J, et al. Cardiac-Resynchronization Therapy in Heart Failure with a Narrow QRS Complex. N Engl J Med 2013; 369: 1395-1405.

45. Beshai JF, Grimm RA, Nagueh SF, Baker JH, Beau SL, Greenberg SM, et al. Cardiac-resynchronization therapy in heart failure with narrow QRS complexes. N Engl J Med 2007; 357: 2461-2471.

46. De Boeck BWL, Meine M, Leenders GE, Teske AJ, Van Wessel H, Kirkels JH, et al. Practical and conceptual limitations of tissue Doppler imaging to predict reverse remodelling in cardiac resynchronisation therapy. Eur J Heart Failure 2008; 10: 281-290.

47. Lumens J, Tayal B, Walmsley J, Delgado-Montero A, Huntjens PR, Saba S, et al. Differentiating the electromechanical substrate responsive to cardiac resynchronization therapy from non-electrical dyssynchrony substrates by computer-assisted regional strain analysis. Circulation Cardiovasc Imag 2015; 8: e003744.

48. Parsai C, Bijnens B, Sutherland GR, Baltabaeva A, Claus P, Marciniak M, et al. Toward understanding response to cardiac resynchronization therapy: left ventricular dyssynchrony is only one of multiple mechanisms. Eur Heart J 2009; 30: 940-949.

49. Stankovic I, Prinz C, Ciarka A, Daraban AM, Kotrc M, Aarones M, et al. Relationship of visually assessed apical rocking and septal flash to response and long-term survival following cardiac resynchronization therapy (PREDICT-CRT). Eur Heart J Cardiovasc Imaging 2016; 17: 262-269.

50. Maass AH, Vernooy K, Wijers SC, van 't Sant J, Cramer MJ, Meine M, et al. Refining success of cardiac resynchronization therapy using a simple score predicting the amount of reverse ventricular remodelling: results from the Markers and Response to CRT (MARC) study. Europace 2018; 20: e1e10.

51. Risum N, Strauss D, Sogaard P, Loring Z, Hansen TF, Bruun NE, et al. Left bundle-branch block: The relationship between electrocardiogram electrical activation and echocardiography mechanical contraction. Am Heart J 2013; 166: 340-348.

52. Bilchick KC, Kuruvilla S, Hamirani YS, Ramachandran R, Clarke SA, Parker KM, et al. Impact of mechanical activation, scar, and electrical timing on cardiac resynchronization therapy response and clinical outcomes. J Am Coll Cardiol 2014; 63: 1657-1666.

53. Leenders GE, De Boeck BW, Teske AJ, Meine M, Bogaard MD, Prinzen FW, et al. Septal rebound stretch is a strong predictor of outcome after cardiac resynchronization therapy. J Card Fail 2012; 18: 404-412.

54. van Oosterhout MF, Prinzen FW, Arts T, Schreuder JJ, Vanagt WY, Cleutjens JP, et al. Asynchronous electrical activation induces asymmetrical hypertrophy of the left ventricular wall. Circulation 1998; 98: 588-595.

55. Steendijk P, Tulner SA, Bax JJ, Oemrawsingh PV, Bleeker GB, van Erven L, et al. Hemodynamic effects of long-term cardiac resynchronization therapy: analysis by pressure-volume loops. Circulation 2006; 113: 1295-1304. 
56. Prinzen FW, Hunter WC, Wyman BT, McVeigh ER. Mapping of regional myocardial strain and work during ventricular pacing: experimental study using Magnetic Resonance Imaging tagging. J Am Coll Cardiol 1999; 33: 1735-1742.

57. Russell K, Eriksen M, Aaberge L, Wilhelmsen N, Skulstad H, Remme EW, et al. A novel clinical method for quantification of regional left ventricular pressure-strain loop area: a non-invasive index of myocardial work. Eur Heart J 2012; 33: 724-733

58. Russell K, Eriksen M, Aaberge L, Wilhelmsen N, Skulstad H, Gjesdal O, et al. Assessment of wasted myocardial work: a novel method to quantify energy loss due to uncoordinated left ventricular contractions. Am J Physiol Heart Circ Physiol 2013; 305: H996-1003.

59. Aalen J, Storsten P, Remme EW, Sirnes PA, Gjesdal O, Larsen CK, et al. Afterload Hypersensitivity in Patients With Left Bundle Branch Block. JACC Cardiovasc Imaging 2019; 12: 967-977.

60. Mills RW, Cornelussen RN, Mulligan LJ, Strik M, Rademakers LM, Skadsberg ND, et al. Left ventricular septal and left ventricular apical pacing chronically maintain cardiac contractile coordination, pump function and efficiency. Circulation: Arrhythm Electrophysiol 2009; 2: 571-579.

61. Nelson GS, Berger RD, Fetics BJ, Talbot M, Spinelli C, Hare JM, et al. Left ventricular or biventricular pacing improves cardiac function at diminished energy cost in patients with dilated cardiomyopathy and left bundle-branch block. Circulation 2000; 102: 3053-3059.

62. Lindner O, Vogt J, Kammeier A, Wielepp P, Holzinger J, Baller D, et al. Effect of cardiac resynchronization therapy on global and regional oxygen consumption and myocardial blood flow in patients with non-ischaemic and ischaemic cardiomyopathy. Eur Heart J 2005; 26: 70-76.

63. Kyriacou A, Pabari PA, Mayet J, Peters NS, Davies DW, Lim PB, et al. Cardiac resynchronization therapy and $\mathrm{AV}$ optimization increase myocardial oxygen consumption, but increase cardiac function more than proportionally. Int J Cardiol 2014; 171: 144-152.

64. Knaapen P, van Campen LM, de Cock CC, Gotte MJ, Visser CA, Lammertsma AA, et al. Effects of cardiac resynchronization therapy on myocardial perfusion reserve. Circulation 2004; 110: 646-651.

65. Vecera J, Penicka M, Eriksen M, Russell K, Bartunek J, Vanderheyden M, et al. Wasted septal work in left ventricular dyssynchrony: a novel principle to predict response to cardiac resynchronization therapy. Eur Heart J Cardiovasc Imaging 2016; 17: 624-632.

66. Zweerink A, de Roest GJ, Wu L, Nijveldt R, de Cock CC, van Rossum AC, et al. Prediction of Acute Response to Cardiac Resynchronization Therapy by Means of the Misbalance in Regional Left Ventricular Myocardial Work. J Card Fail 2016; 22: 133-142.

67. Vernooy K, Verbeek XA, Peschar M, Crijns HJ, Arts T, Cornelussen RN, et al. Left bundle branch block induces ventricular remodelling and functional septal hypoperfusion. European heart journal 2005; 26: 91-98.

68. Vernooy K, Cornelussen RN, Verbeek XA, Vanagt WY, van Hunnik A, Kuiper M, et al. Cardiac resynchronization therapy cures dyssynchronopathy in canine left bundle-branch block hearts. European heart journal 2007; 28: 2148-2155.

69. Amitzur G, Manor D, Pressman A, Adam D, Hammerman H, Shofti R, et al. Modulation of the arterial coronary blood flow by asynchronous activation with ventricular pacing. Pacing Clin Electrophysiol 1995; 18: 697-710. 
70. Kirk JA, Kass DA. Cellular and Molecular Aspects of Dyssynchrony and Resynchronization. Heart Fail Clin 2017; 13: 29-41.

71. Wiegerinck RF, Schreurs R, Prinzen FW. Pathophysiology of dyssynchrony: of squirrels and broken bones. Neth Heart J 2016; 24: 4-10.

72. Vanderheyden M, Vrints $\mathrm{C}$, Bartunek J. The molecular fingerprint of cardiac dyssynchrony and cardiac resynchronization therapy. Heart Fail Rev 2011; 16: 227-233.

73. van Berlo JH, Maillet M, Molkentin JD. Signaling effectors underlying pathologic growth and remodeling of the heart. J Clin Invest 2013; 123: 37-45.

74. Heineke J, Molkentin JD. Regulation of cardiac hypertrophy by intracellular signalling pathways. Nat Rev Mol Cell Biol 2006; 7: 589-600.

75. Blaauw E, Lorenzen-Schmidt I, Babiker FA, Munts C, Prinzen FW, Snoeckx LH, et al. Stretch-induced upregulation of connective tissue growth factor in rabbit cardiomyocytes. J Cardiovasc Trans/ Res 2013; 6: 861-869.

76. Blaauw E, van Nieuwenhoven FA, Willemsen P, Delhaas T, Prinzen FW, Snoeckx LH, et al. Stretchinduced hypertrophy of isolated adult rabbit cardiomyocytes. Am J Physiol Heart Circ Physiol 2010; 299: $\mathrm{H} 780-787$.

77. Herum KM, Choppe J, Kumar A, Engler AJ, McCulloch AD. Mechanical regulation of cardiac fibroblast profibrotic phenotypes. Mol Biol Cell 2017; 28: 1871-1882.

78. Strik M, van Middendorp LB, Vernooy K. Animal models of dyssynchrony. J Cardiovasc Transl Res 2012; 5: 135-145.

79. Spragg DD, Leclercq C, Loghmani M, Faris OP, Tunin RS, DiSilvestre D, et al. Regional alterations in protein expression in the dyssynchronous failing heart. Circulation 2003; 108: 929-932.

80. Nishijima Y, Sridhar A, Viatchenko-Karpinski S, Shaw C, Bonagura JD, Abraham WT, et al. Chronic cardiac resynchronization therapy and reverse ventricular remodeling in a model of nonischemic cardiomyopathy. Life Sci 2007; 81: 1152-1159.

81. Aiba T, Hesketh GG, Barth AS, Liu T, Daya S, Chakir K, et al. Electrophysiological consequences of dyssynchronous heart failure and its restoration by resynchronization therapy. Circulation 2009; 119: 1220-1230.

82. Akar FG, Spragg DD, Tunin RS, Kass DA, Tomaselli GF. Mechanisms underlying conduction slowing and arrhythmogenesis in nonischemic dilated cardiomyopathy. Circ Res 2004; 95: 717-725.

83. St. John Sutton MG, Plappert T, Abraham WT, Smith AL, DeLurgio DB, Leon AR, et al. Effect of cardiac resynchronization therapy on left ventricular size and function in chronic heart failure. Circulation 2003; 107: 1985-1990.

84. Yu CM, Bleeker GB, Fung JW, Schalij MJ, Zhang Q, van der Wall EE, et al. Left ventricular reverse remodeling but not clinical improvement predicts long-term survival after cardiac resynchronization therapy. Circulation 2005; 112: 1580 - 1586.

85. D'Ascia C, Cittadini A, Monti MG, Riccio G, Sacca L. Effects of biventricular pacing on interstitial remodelling, tumor necrosis factor-alpha expression, and apoptotic death in failing human myocardium. European heart journal 2006; 27: 201-206. 
86. Orrego CM, Nasir N, Oliveira GH, Flores-Arredondo JH, Cordero-Reyes AM, Loebe M, et al. Cellular evidence of reverse cardiac remodeling induced by cardiac resynchronization therapy. Congest Heart Fail 2011; 17: 140-146.

87. Zhang Q, Fung JW, Auricchio A, Chan JY, Kum LC, Wu LW, et al. Differential change in left ventricular mass and regional wall thickness after cardiac resynchronization therapy for heart failure. Eur Heart J 2006; 27: 1423-1430.

88. Soliman OI, Geleijnse ML, Theuns DA, Nemes A, Vletter WB, van Dalen BM, et al. Reverse of left ventricular volumetric and structural remodeling in heart failure patients treated with cardiac resynchronization therapy. Am J Cardiol 2008; 101: 651-657.

89. Schmitz B, De Maria R, Gatsios D, Chrysanthakopoulou T, Landolina M, Gasparini M, et al. Identification of genetic markers for treatment success in heart failure patients: insight from cardiac resynchronization therapy. Circ Cardiovasc Genet 2014; 7: 760-770.

90. De Maria R, Landolina M, Gasparini M, Schmitz B, Campolo J, Parolini M, et al. Genetic variants of the renin-angiotensin-aldosterone system and reverse remodeling after cardiac resynchronization therapy. J Card Fail 2012; 18: 762-768.

91. van Middendorp LB, Kuiper M, Munts C, Wouters P, Maessen JG, van Nieuwenhoven FA, et al. Local microRNA-133a downregulation is associated with hypertrophy in the dyssynchronous heart. ESC Heart Fail 2017; 4: 241-251.

92. Lin JM, Lai LP, Lin CS, Chou NK, Chiu CY, Lin JL. Left ventricular extracellular matrix remodeling in dogs with right ventricular apical pacing. J Cardiovasc Electrophysiol 2010; 21: 1142-1149.

93. Wang J, Gong X, Chen H, Qin S, Zhou N, Su Y, et al. Effect of Cardiac Resynchronization Therapy on Myocardial Fibrosis and Relevant Cytokines in a Canine Model With Experimental Heart Failure. $J$ Cardiovasc Electrophysiol 2017; 28: 438-445.

94. Spragg DD, Akar FG, Helm RH, Tunin RS, Tomaselli GF, Kass DA. Abnormal conduction and repolarization in late-activated myocardium of dyssynchronously contracting hearts. Cardiovascular research 2005; 67: 77-86.

95. Vanderheyden M, Mullens W, Delrue L, Goethals M, de Bruyne B, Wijns W, et al. Myocardial gene expression in heart failure patients treated with cardiac resynchronization therapy responders versus nonresponders. J Am Coll Cardiol 2008; 51: 129-136.

96. Chakir K, Daya SK, Tunin RS, Helm RH, Byrne MJ, Dimaano VL, et al. Reversal of global apoptosis and regional stress kinase activation by cardiac resynchronization. Circulation 2008; 117: 13691377.

97. Marin-Garcia J, Goldenthal MJ, Damle S, Pi Y, Moe GW. Regional distribution of mitochondrial dysfunction and apoptotic remodeling in pacing-induced heart failure. Journal of cardiac failure 2009; 15: 700-708.

98. Klug D, Boule S, Wissocque L, Montaigne D, Marechal X, Hassoun SM, et al. Right ventricular pacing with mechanical dyssynchrony causes apoptosis interruptus and calcium mishandling. Can J Cardiol 2013; 29: 510-518.

99. Reddy VY, Neuzil P, Taborsky M, Kralovec S, Sediva L, Ruskin JN. Images in cardiovascular medicine. Electroanatomic mapping of cardiac resynchronization therapy. Circulation 2003; 107: 2761-2763. 
100. Akar FG, Nass RD, Hahn S, Cingolani E, Shah M, Hesketh GG, et al. Dynamic changes in conduction velocity and gap junction properties during development of pacing-induced heart failure. Am $J$ Physiol Heart Circ Physiol 2007; 293: H1223-1230.

101. Smith JH, Green CR, Peters NS, Rothery S, Severs NJ. Altered patterns of gap junction distribution in ischemic heart disease. An immunohistochemical study of human myocardium using laser scanning confocal microscopy. Am J Pathol 1991; 139: 801-821.

102. Josephson ME, Anter E. Substrate Mapping for Ventricular Tachycardia: Assumptions and Misconceptions. JACC Clin Electrophysiol 2015; 1: 341-352.

103. Maguy A, Le Bouter S, Comtois P, Chartier D, Villeneuve L, Wakili R, et al. Ion channel subunit expression changes in cardiac Purkinje fibers: a potential role in conduction abnormalities associated with congestive heart failure. Circ Res 2009; 104: 1113-1122.

104. Valdivia CR, Chu WW, Pu J, Foell JD, Haworth RA, Wolff MR, et al. Increased late sodium current in myocytes from a canine heart failure model and from failing human heart. J Mol Cell Cardiol 2005; 38: $475-483$.

105. Kaab S, Nuss HB, Chiamvimonvat N, O'Rourke B, Pak PH, Kass DA, et al. lonic mechanism of action potential prolongation in ventricular myocytes from dogs with pacing-induced heart failure. Circ Res 1996; 78: 262-273.

106. Shvilkin A, Huang HD, Josephson ME. Cardiac memory: diagnostic tool in the making. Circ Arrhythm Electrophysiol 2015; 8: 475-482.

107. Sosunov EA, Anyukhovsky EP, Rosen MR. Altered ventricular stretch contributes to initiation of cardiac memory. Heart Rhythm 2008; 5: 106-113.

108. Shvilkin A, Bojovic B, Vajdic B, Gussak I, Zimetbaum P, Josephson ME. Vectorcardiographic determinants of cardiac memory during normal ventricular activation and continuous ventricular pacing. Heart Rhythm 2009; 6: 943-948.

109. Wecke L, Rubulis A, Lundahl G, Rosen MR, Bergfeldt L. Right ventricular pacing-induced electrophysiological remodeling in the human heart and its relationship to cardiac memory. Heart Rhythm 2007; 4: 1477-1486.

110. Wecke L, van Deursen CJ, Bergfeldt L, Prinzen FW. Repolarization changes in patients with heart failure receiving cardiac resynchronization therapy-signs of cardiac memory. $J$ Electrocardiol 2011; 44: 590-598.

111. Plotnikov AN, Yu H, Geller JC, Gainullin RZ, Chandra P, Patberg KW, et al. Role of L-type calcium channels in pacing-induced short-term and long-term cardiac memory in canine heart. Circulation 2003; 107: 2844-2849.

112. Patberg KW, Plotnikov AN, Quamina A, Gainullin RZ, Rybin A, Danilo Jr P, et al. Cardiac memory is associated with decreased levels of the transcriptional factor CREB modulated by angiotensin II and Calcium. Circ Res 2003; 93: 472-478.

113. Costard-Jäckle A, Goetsch B, Antz M, Franz MR. Slow and long-lasting modulation of myocardial repolarization produced by ectopic activation in isolated rabbit hearts: Evidence for cardiac "memory". Circulation 1989; 80: 1412-1420.

114. Jeyaraj D, Wilson LD, Zhong J, Flask C, Saffitz JE, Deschênes I, et al. Mechanoelectrical feedback as novel mechanism of cardiac electrical remodeling. Circulation 2007; 115: 3145-3155. 
115. Opthof T, Janse MJ, Meijborg VM, Cinca J, Rosen MR, Coronel R. Dispersion in ventricular repolarization in the human, canine and porcine heart. Prog Biophys Mol Biol 2016; 120: 222-235.

116. Shvilkin A, Bojovic B, Vajdic B, Gussak I, Ho KK, Zimetbaum P, et al. Vectorcardiographic and electrocardiographic criteria to distinguish new and old left bundle branch block. Heart Rhythm 2010; 7: 1085-1092.

117. Engels EB, Poels TT, Houthuizen P, de Jaegere PPT, Maessen JG, Vernooy K, et al. Electrical remodelling in patients with iatrogenic left bundle branch block. Europace 2016; 18(suppl 4): iv44iv52.

118. Maffessanti F, Wanten J, Potse M, Regoli F, Caputo ML, Conte G, et al. The relation between local repolarization and T-wave morphology in heart failure patients. Int J Cardiol 2017; 241: 270-276.

119. Hermeling E, Delhaas T, Prinzen FW, Kuijpers NHL. Mechano electrical feedback explains T-wave morphology and optimizes cardiac pump function: insight from a multiscale model. Prog Biophys Mol Biol 2012; 110: 359-371.

120. Kuijpers NH, Hermeling E, Lumens J, Eikelder HT, Delhaas T, Prinzen FW. Mechano-electrical coupling as framework for understanding functional remodeling during LBBB and CRT. Am J Physiol Heart Circ Physiol 2014; 306: H1644-1659.

121. Lieberman R, Padeletti L, Schreuder J, Jackson K, Michelucci A, Colella A, et al. Ventricular pacing lead location alters systemic hemodynamics and left ventricular function in patients with and without reduced ejection fraction. J Am Coll Cardiol 2006; 48: 1634-1641.

122. Nahlawi M, Waligora M, Spies SM, Bonow RO, Kadish AH, Goldberger JJ. Left ventricular function during and after right ventricular pacing. J Am Coll Cardiol 2004; 44: 1883-1888.

123. Kass DA, Chen C-H, Curry C, Talbot M, Berger R, Fetics B, et al. Improved left ventricular mechanics from acute VDD pacing in patients with dilated cardiomyopathy and ventricular conduction delay. Circulation 1999; 99: 1567-1573.

124. Duckett SG, Ginks M, Shetty AK, Bostock J, Gill JS, Hamid S, et al. Invasive acute hemodynamic response to guide left ventricular lead implantation predicts chronic remodeling in patients undergoing cardiac resynchronization therapy. J Am Coll Cardiol 2011; 58: 1128-1136.

125. Delnoy PP, Ottervanger JP, Luttikhuis HO, Nicastia DM, Elvan A, Misier AR, et al. Sustained benefit of cardiac resynchronization therapy. J Cardiovasc Electrophysiol 2007; 18: 298-302.

126. Salameh A, Dhein S, Blanke K, Rastan A, Hiyasat B, Dietze A, et al. Right or left ventricular pacing in young minipigs with chronic atrioventricular block: long-term in vivo cardiac performance, morphology, electrophysiology, and cellular biology. Circulation 2012; 125: 2578-2587.

127. Schmidt U, Hajjar RJ, Helm PA, Kim CS, Doye AA, Gwathmey JK. Contribution of abnormal sarcoplasmic reticulum ATPase activity to systolic and diastolic dysfunction in human heart failure. $J$ Mol Cell Cardiol 1998; 30: 1929-1937.

128. Iyengar S, Haas G, Lamba S, Orsinelli DA, Babu GJ, Ferketich AK, et al. Effect of cardiac resynchronization therapy on myocardial gene expression in patients with nonischemic dilated cardiomyopathy. Journal of cardiac failure 2007; 13: 304-311.

129. Mullens W, Bartunek J, Tang WH, Delrue L, Herbots L, Willems R, et al. Early and late effects of cardiac resynchronization therapy on force-frequency relation and contractility regulating gene expression in heart failure patients. Heart Rhythm 2008; 5: 52-59. 
130. Lin JM, Lai LP, Chou NK, Lin JL. Spatial heterogeneity of protein expression induced by dyssynchronous right ventricular pacing in the left ventricle of dogs with preserved systolic function. Journal of cardiac failure 2010; 16: 700-706.

131. Crossman DJ, Jayasinghe ID, Soeller C. Transverse tubule remodelling: a cellular pathology driven by both sides of the plasmalemma? Biophys Rev 2017; 9: 919-929.

132. Sachse FB, Torres NS, Savio-Galimberti E, Aiba T, Kass DA, Tomaselli GF, et al. Subcellular structures and function of myocytes impaired during heart failure are restored by cardiac resynchronization therapy. Circ Res 2012; 110: 588-597.

133. Li H, Lichter JG, Seidel T, Tomaselli GF, Bridge JH, Sachse FB. Cardiac Resynchronization Therapy Reduces Subcellular Heterogeneity of Ryanodine Receptors, T-Tubules, and Ca2+ Sparks Produced by Dyssynchronous Heart Failure. Circulation Heart failure 2015; 8: 1105-1114.

134. Bhargava V, Shabetai R, Mathiäsen RA, Dalton N, Hunter JJ, Ross J. Loss of adrenergic control of the force-frequency relation in heart failure secondary to idiopathic or ischemic cardiomyopathy. The American journal of cardiology 1998; 81: 1130-1137.

135. Vanderheyden M, Mullens W, Delrue L, Goethals M, Verstreken S, Wijns W, et al. Endomyocardial upregulation of beta1 adrenoreceptor gene expression and myocardial contractile reserve following cardiac resynchronization therapy. Journal of cardiac failure 2008; 14: 172-178.

136. Ungerer M, Böhm M, Elce JS, Erdmann E, Lohse MJ. Altered Expression of Beta-Adrenergic Receptor Kinase and Beta1-Adrenergic Receptors in the Failing Human Heart. Circulation 1993; 87: 454-463.

137. Chakir K, Depry C, Dimaano VL, Zhu WZ, Vanderheyden M, Bartunek J, et al. Galphas-biased beta2-adrenergic receptor signaling from restoring synchronous contraction in the failing heart. $\mathrm{Sci}$ Transl Med 2011; 3: 100ra188.

138. Chakir K, Daya SK, Aiba T, Tunin RS, Dimaano VL, Abraham TP, et al. Mechanisms of enhanced beta-adrenergic reserve from cardiac resynchronization therapy. Circulation 2009; 119: 1231-1240.

139. Kirk JA, Holewinski RJ, Kooij V, Agnetti G, Tunin RS, Witayavanitkul N, et al. Cardiac resynchronization sensitizes the sarcomere to calcium by reactivating GSK-3beta. J Clin Invest 2014; 124: 129-138.

140. Leenders GE, Lumens J, Cramer MJ, De Boeck BWL, Doevendans PA, Delhaas T, et al. Septal deformation patterns delineate mechanical dyssynchrony and regional differences in contractility: analysis of patient data using a computer model. Circulation: Heart Failure 2012; 5: 87-96. 



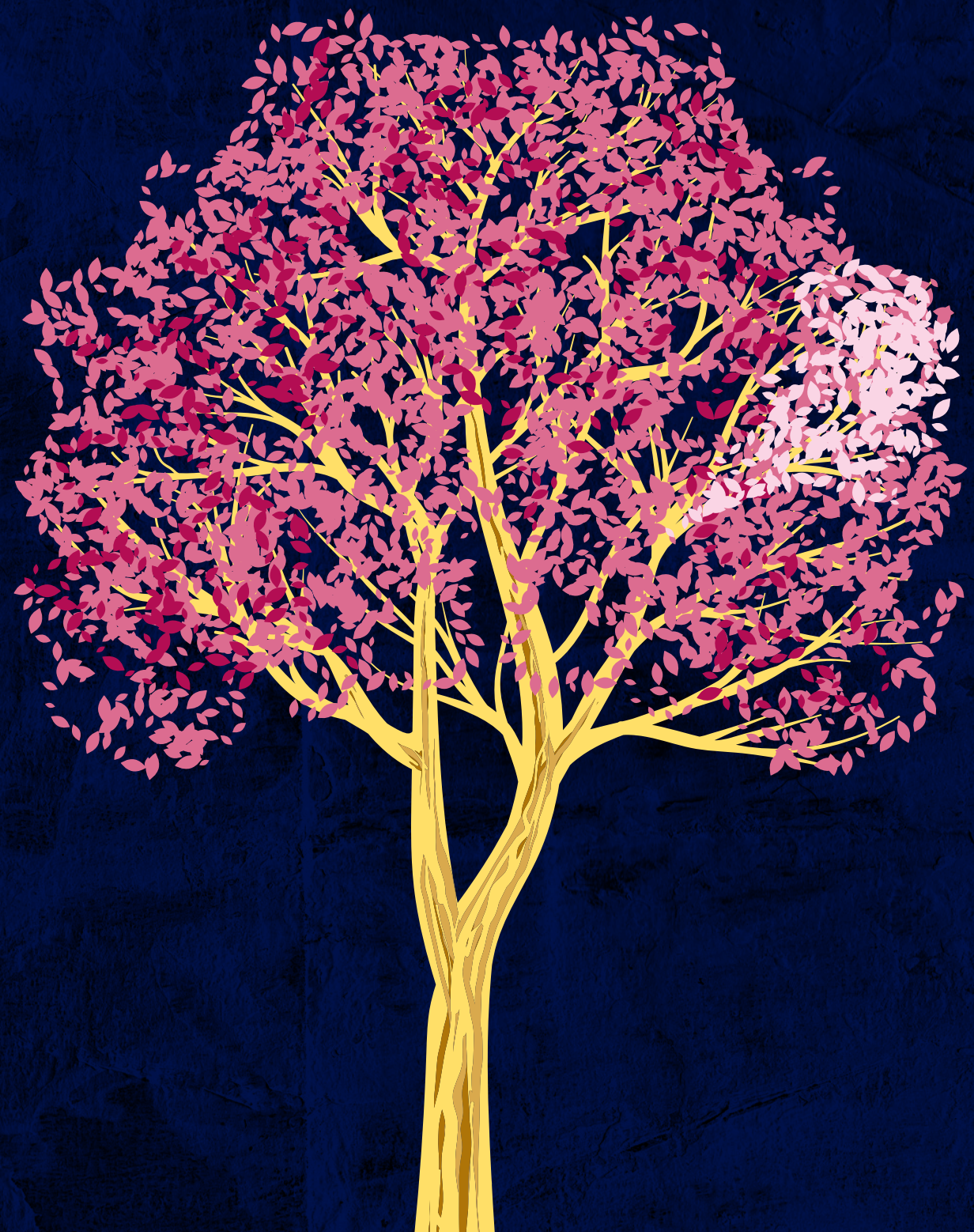




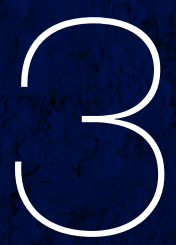

\section{Time course of repolarization changes after start of cardiac resynchronization therapy}

Nienke J. Verzaal ${ }^{1 \star}$, Caroline J.M. van Deursen² ${ }^{2 \star}$ Simone Pezzuto ${ }^{3}$, Liliane Wecke ${ }^{4}$, Wouter van Everdingen ${ }^{5}$, Angelo Auricchio ${ }^{3,6}$, Kevin Vernooy ${ }^{2}$, Tammo Delhaas ${ }^{7}$ and Frits W. Prinzen ${ }^{1}$

\footnotetext{
1 Department of Physiology, Maastricht University, Maastricht, The Netherlands

${ }^{2}$ Department of Cardiology, Maastricht University Medical Centre, Maastricht, The Netherlands

${ }^{3}$ Centre for Computational Medicine in Cardiology, Institute of Computational Science, Università della Svizzera Italiana Lugano, Switzerland

${ }^{4}$ Heart Clinic, Capio St. Göran's Hospital, Stockholm, Sweden

${ }^{5}$ Department of Radiology and Nuclear Medicine, Radboudumc, Nijmegen, The Netherlands

${ }^{6}$ Department of Cardiology, Cardiocentro Ticino, Lugano, Switzerland

${ }^{7}$ Department of Biomedical Engineering, Maastricht University, Maastricht, The Netherlands

* These authors contributed equally.
} 


\section{Abstract}

Aims While cardiac resynchronization therapy (CRT) clearly resynchronizes ventricular depolarization and thereby improves cardiac pump function in patients with dyssynchronous heart failure, its effect on ventricular repolarization is not entirely clear. This study explored the effects of CRT on repolarization by measuring T wave morphology changes after CRT, relating the changes to mechanical remodelling and by interpreting these results using a patientspecific computational model.

Methods In 33 CRT recipients, electrocardiographic and echocardiographic measurements were performed prior to device implantation and repeatedly between 1 day and 6 months of CRT. Measurements were obtained both during CRT (CRT-ON) and during brief interruption of CRT (CRT-OFF). Twelve-lead electrocardiograms were converted to vectorcardiograms and QRS area and T area were calculated, along with JTc interval, T peak-to-end interval $\left(T_{p-e}\right)$ and precordial QT dispersion. Results were interpreted using a patient-specific propagation and ECG model of the ventricles.

Results CRT immediately and persistently decreased QRS area and T area, whereas it increased JTc interval. CRT did not significantly influence $T_{p-e}$ and precordial QT dispersion. During CRTOFF, T area, $T_{p-e}$ and JTc interval increased significantly, reaching plateau levels 5-14 days after starting CRT. Left ventricular ejection fraction and systolic septal strain increased between 2 weeks and 6 months of CRT. The observed changes in the T wave were largely reproduced in the patient-specific model when assuming an inverse relation between CRT-induced change in activation time and adaptation in action potential duration. The latter changes resulted in a reduction in dispersion of repolarization during chronic CRT as compared to acute CRT.

Conclusion CRT causes differential changes in various $T$ wave markers that occur relatively fast ( $<2$ weeks) after onset of CRT. These repolarization changes precede mechanical remodelling and indicate a reduction in dispersion of repolarization during longer-lasting CRT. 


\section{Introduction}

Cardiac resynchronization therapy (CRT) is an important therapy for patients with dyssynchronous heart failure and significantly reduces mortality and heart failure hospitalizations ${ }^{1}$. This beneficial effect is clearly linked to resynchronization of electrical activation, recoordination of contraction and accompanying improvement in pump function and cardiac reserve.

While depolarization changes after CRT have been studied in considerable detail, less is known about changes in repolarization caused by CRT. A few studies in both animal tissues and humans reported increased dispersion of repolarization during CRT or epicardial pacing ${ }^{2,3}$. A computer modelling study predicted increased transmural dispersion of repolarization directly underneath the pacing electrodes during CRT, while the effects on average transmural dispersion of repolarization appeared modest ${ }^{4}$.

Increased dispersion of repolarization has been linked to arrhythmias, leading to the suggestion that CRT may be pro-arrythmic ${ }^{2}$. However, single-centre studies did not observe a change in occurrence of arrhythmia after upgrade from implantable cardioverter defibrillator to $\mathrm{CRT}^{5,6}$. Furthermore, the arrhythmia risk was not significantly different between the CRT-ON and CRTOFF groups in the REVERSE trial ${ }^{7}$. Actually, the REVERSE and MADIT trials and several smaller studies demonstrated that CRT leads to fewer arrhythmias in CRT responders ${ }^{5,7-9}$.

A potential explanation for the discrepant findings in the small, acute studies and the larger clinical studies may be that repolarization is known to adapt to an altered activation sequence, a phenomenon that has been demonstrated by brief interruption of ventricular pacing ${ }^{10,11}$. This adaptation of repolarization, often referred to as "cardiac memory", may lead to reduced dispersion of repolarization over time ${ }^{12}$. The time course at which this phenomenon occurs has not yet been studied extensively in patients receiving $C R T^{10,13}$.

It was the aim of the present study to investigate the time course and extent of changes in T wave markers of repolarization in patients after the start of CRT. To this purpose, electrocardiographic (ECG) and vectorcardiographic (VCG) analyses were used, providing variables like QTC and JTC interval, $T$ wave area, time interval between peak and end of the $T$ wave $\left(T_{p-e}\right)$ and precordial QT dispersion. Moreover, echocardiography was performed, to assess mechanical response to CRT and its time course relative to the electrical changes. For more detailed appreciation of the remodelling that underlies changes in the $T$ wave, measurements were performed at multiple time points after start of CRT, both during CRT (CRT-ON) and after temporary interruption of CRT (CRT-OFF), the latter being used to asses "cardiac memory". Finally, a 3D computational model of ventricular activation and repolarization, including "cardiac memory" for action potential duration (APD) was implemented to mechanistically interpret the results. 


\section{Materials and methods}

\section{Patients}

Patients referred to Maastricht University Medical Centre who were (1) 18 years of age or older; (2) had an indication for CRT; (3) did not have any known condition that could limit life expectancy to less than 6 months as of referral and (4) were capable of giving informed consent were asked to participate. The study conforms to the Declaration of Helsinki. The protocol was approved by the ethics committee of Maastricht University Medical Centre (project number 10-2-090) and all patients gave their written informed consent to participate before inclusion.

\section{Study protocol}

Baseline 12-lead ECG was obtained at most 18 days prior to implantation of a CRT device. Follow-up ECGs were obtained 1 day, 5 days (range: 3-9 days), 2 weeks (8-16 days), 1 month (28-41 days), 3 months (2-4 months) and 6 months (6-8 months) after implantation, both during CRT-ON and while the CRT device was briefly switched off (CRT-OFF).

Echocardiography was performed before implantation (range: 3 months to 1 day before implantation) and 1 day, 2 weeks (8-19 days) and 6 months (6-8 months) after implantation. If no 1-day post-operative echocardiogram was available, another early post-operative echocardiogram was analysed (range 3-7 days). Post-operative echocardiographic measurements were obtained with both CRT-ON and CRT-OFF.

\section{VCG analysis}

ECGs were extracted from the MUSE Cardiology Information system (GE Healthcare, Chicago, US). Using custom-written MATLAB software, VCGs were synthesized from the ECGs using the Kors matrix and subsequently, semi-automatic analysis was performed, as described previously ${ }^{14}$. Maximum QRS and T vectors were defined as the maximum distances between the origin of the VCG loop and a point on the 3D QRS and T loops, respectively. The size and direction of these maximal vectors were expressed by the vector amplitude, azimuth (angle in the transverse plane, for QRS azimuth, the values were expressed on a full circle $\left(360^{\circ}\right)$ ) and elevation (angle in the craniocaudal plane). Furthermore, the area under the curve of the QRS complex and $\mathrm{T}$ wave in the $\mathrm{X}, \mathrm{Y}$, and $\mathrm{Z}$ direction was determined by numerical integration from the beginning to the end of the QRS complex or T wave, respectively (Figure 3, General introduction). Subsequently, the three areas were summed to determine the total area of the QRS and T loops, using these formulas:

$$
Q R S_{\text {area }}=\sqrt{Q R S_{\text {area } x}^{2}+\mathrm{QRS}_{\text {area }, y}^{2}+\mathrm{QRS}_{\text {area }, \mathrm{z}}^{2}} \text { and } T_{\text {area }}=\sqrt{T_{\text {area }, \mathrm{x}}^{2}+\mathrm{T}_{\text {area }, y}^{2}+\mathrm{T}_{\text {area }, \mathrm{z}}^{2}}
$$


The different effects of CRT on depolarization and repolarization were further explored by calculating the QRS-T area ratio.

QT interval was measured automatically using the tangent method as described previously ${ }^{15}$ and subsequently corrected for heart rate (QTc) using Fridericia's formula, since this formula has been shown to be superior to Bazett's formula ${ }^{16}$. Median $T_{p-e}$ for all leads and precordial QT dispersion (standard deviation of start $Q$ to precordial $T$ ends) were determined. JTC was determined by subtracting QRS width from the QT interval and subsequently applying Fridericia's formula.

\section{Echocardiography}

Echocardiograms were obtained using an iE33 system (Philips Medical Systems, Best, the Netherlands). Left ventricular end-systolic volume (LVESV) and left ventricular ejection fraction (LVEF) were calculated using the biplane method of disks (modified Simpson's rule) or monoplane in the apical 4-chamber window if the apical 2-chamber view was of insufficient quality for reliable analysis. CRT response was defined as a reduction of LVESV $\geq 15 \%$ comparing the baseline to the 6-month CRT-ON echocardiogram.

Interventricular mechanical delay (IVMD) was defined as the time difference between opening of the pulmonary valve and aortic valve on the pulsed wave Doppler echocardiogram. Using speckle tracking analysis (QLAB version 8.1, Philips Medical Systems, Bothell, WA, USA) septal strain was determined, since this focusses on the ventricular wall that is visualized best and shows clear paradoxical motion before $\mathrm{CRT}^{17}$. Systolic septal strain (SS) was determined as the most negative strain value in systole. Systolic rebound stretch of the septum (SRS), a strong indicator of CRT response, was defined as the total amount of stretch during systole that occurred after initial shortening ${ }^{18}$.

\section{In silico modelling}

A previously described eikonal model with ECG simulation was adopted for simulating patientspecific ventricular activation and depolarization ${ }^{19}$. ECG and magnetic resonance imaging (MRI) data of a patient, participating in an earlier study performed in Lugano, were used to this purpose ${ }^{20}$. The eikonal equation modelled the spread of action potential from a given initial set of earliest activation sites (EASs) throughout the whole active myocardium, with a heterogeneous, direction-dependent conduction velocity. The spatiotemporal dynamic of the transmembrane potential $V_{m}(x, t)$ was modelled by setting the onset $\tau_{0}(x)$ and the duration $\tau_{1}(x)$ of an action potential (AP) template based on the formula:

$$
V_{m}(x, t)=V_{0}+\frac{V_{1}-V_{0}}{2}\left(\tanh \left(\frac{t-\tau_{0}(x)}{\epsilon_{0}}\right)-\tanh \left(\frac{t-\tau_{0}(x)-\tau_{1}(x)}{\epsilon_{1}(x)}\right)\right)
$$


The following parameters were fixed for all simulations:

$$
V_{0}=-85 \mathrm{mV}, V_{1}=30 \mathrm{mV}, \epsilon_{0}=1 \mathrm{~ms}
$$

The corresponding surface ECG was computed from the transmembrane potential using the lead field theory ${ }^{21}$. The cardiac MRI-derived and at 1-mm resolution segmented heart-torso anatomy included ventricles, atria, blood cavities, lungs, and skeletal muscles. Ventricular fibres were automatically assigned using a rule-based approach ${ }^{20}$. Intra- and extracellular electric conductivities and surface-to-volume ratio were set according to previous studies ${ }^{19}$. The baseline activation sequence, encoded in $\tau_{0}(x)$, was automatically fitted, in the leastsquares sense, to the QRS complex by adapting the conduction velocity and the sites of earliest activation, whereas the repolarization sequence was adapted from the T wave by adjusting the APD $\tau_{1}(x)$ and repolarization steepness $\epsilon_{1}(x)$ in 6 distinct compartments: endocardium, mid-myocardium and epicardium of the left ventricle (LV) and the right ventricle (RV). Midmyocardium and epicardium respectively covered $30 \%$ and $20 \%$ of the transmural thickness. A 1-mm-thick endocardial layer with fast conduction was included in both the LV and RV. APD90 was defined as the time at which AP reached $90 \%$ of its resting potential during the repolarization phase. Acute CRT was simulated by biventricular pacing, with manually placed leads on LV epicardium and RV apex and no interventricular delay. Repolarization remodelling in chronic CRT was modelled by locally decreasing the APD with $60 \%$ of the difference between depolarization time after and prior to CRT:

$$
\tau_{1}^{\text {chronic }}=\tau_{1}^{\text {base }}-x \cdot\left(\tau_{0}^{\mathrm{CRT}}-\tau_{0}^{\text {base }}\right)
$$

The factor $x$ was varied between 0.5 and 0.8 to find the best match with the measured $T$ wave parameters. The ECGs of 4 scenarios (baseline, acute CRT-ON, chronic CRT-ON and CRT-OFF) were analysed with the same workflow adopted for the clinical cohort (see above). Simulated baseline $T$ area value was multiplied by a normalization factor to match the average baseline $T$ area value in the patient cohort. All other simulated T and QRS area values were multiplied by the same factor.

\section{Statistical analysis}

Statistical analysis of the patient data was performed by fitting a mixed model (GraphPad Prism 8, GraphPad Software, San Diego, USA). This mixed model uses a compound symmetry covariance matrix and is fit using Restricted Maximum Likelihood. We applied the GeisserGreenhouse correction to account for possible violations of the assumption of sphericity. For the assessment of differences between time points we performed a Bonferroni's multiple comparisons test. $P$ values $\leq 0.05$ were considered statistically significant. Data are shown as mean \pm standard deviation (SD) unless otherwise indicated. 


\section{Results}

Thirty-five patients were included in the study. One patient withdrew consent upon second consideration. One patient was excluded from analysis since neither ECGs nor echocardiograms of sufficient quality were available.

For the ECG analysis, 8 patients were excluded since too few ECGs of good quality were available. Therefore, ECG analysis was performed in 25 patients. In 5 patients the echocardiographic apical 2-chamber view was of poor quality. For this reason, the LVEF and LVESV measurements were performed in monoplane 4-chamber views in these 5 patients, and in biplane in the other 28 patients. For IVMD, all remaining 33 patients were studied. For SRS and SS, 32 patients were studied (1 patient excluded because of insufficient image quality).

Mean age of the 33 patients (10 women) who were included in the study was $67 \pm 8$ years. A de novo biventricular device implantation was performed in 24 patients, and 9 patients received an upgrade to a biventricular device because of intrinsic conduction delay $(n=5)$ or right ventricular pacing $(n=4)$. Further patient characteristics can be found in Table 1.

The RV lead was placed in an endocardial apical position in all patients. The LV lead (transvenously inserted in all patients but 1 , in whom the lead was placed surgically) was most commonly implanted in the basal anterolateral $(n=9)$, mid anterolateral $(n=10)$ and the mid inferolateral $(n=8)$ regions (Table 1$)$. 
TABLE 1 Patient characteristics

\begin{tabular}{|c|c|}
\hline Age (years) & $67 \pm 8$ \\
\hline Female (n, \%) & $10(30)$ \\
\hline BMI $\left(\mathbf{k g} / \mathrm{m}^{2}\right)$ & $27 \pm 5$ \\
\hline \multicolumn{2}{|l|}{ NYHA class } \\
\hline I $(n, \%)$ & $6(18)$ \\
\hline II $(\mathrm{n}, \%)$ & $12(36)$ \\
\hline III $(\mathrm{n}, \%)$ & $12(36)$ \\
\hline IV (n, \%) & $3(9)$ \\
\hline Ischemic cardiomyopathy ( $\mathrm{n}, \%$ ) & $19(58)$ \\
\hline$A F(n, \%)$ & $8(24)$ \\
\hline LBBB $(n, \%)$ & $28(85)$ \\
\hline Previous RV pacing (n, \%) & $4(12)$ \\
\hline ACE-inhibitor/ARB (n,\%) & $30(91)$ \\
\hline Beta blocker ( $n, \%)$ & $31(94)$ \\
\hline Aldosterone antagonist (n,\%) & $8(24)$ \\
\hline Loop diuretic $(\mathrm{n}, \%)$ & $21(64)$ \\
\hline CRT responders $(n, \%)$ & $18(60)$ \\
\hline Percentage biventricular pacing (median (IQR)) & $98.8(97.4-99.4)$ \\
\hline \multicolumn{2}{|l|}{ LV lead location $(n, \%)$} \\
\hline Basal inferolateral & $1(3)$ \\
\hline Basal anterolateral & $9(27)$ \\
\hline Mid inferior & $2(6)$ \\
\hline Mid inferolateral & $8(24)$ \\
\hline Mid anterolateral & $10(30)$ \\
\hline Apical lateral & $3(9)$ \\
\hline
\end{tabular}

Variables are shown as mean \pm standard deviation or as number of patients (percentage of patients). $A F=$ atrial fibrillation, $A C E$ = angiotensin converting enzyme, $A R B=$ angiotensin receptor blocker, $B M I=$ body mass index, CRT = cardiac resynchronization therapy, $I Q R=$ interquartile range, $L B B B=$ left bundle branch block, $L V=$ left ventricular, $N Y H A=$ New York Heart Association, RV = right ventricular. 


\section{ECG and VCG parameters}

Figure 1 shows examples of ECG lead V2 of a patient during both CRT-OFF (A) and CRT-ON (B) at various time points. CRT-ON causes immediate amplitude reduction and narrowing of the QRS complex, accompanied by $T$ wave changes. T wave amplitude increases over time during CRT-OFF.

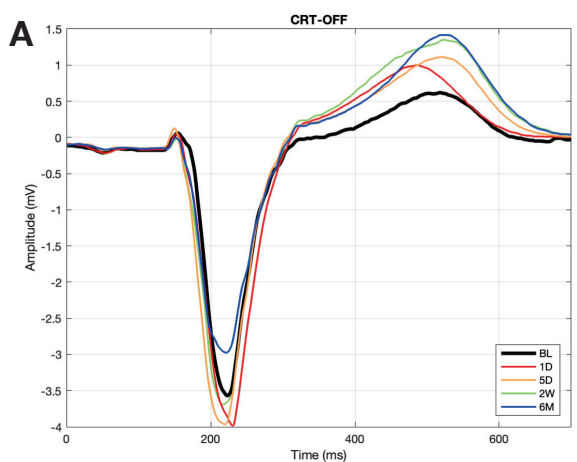

B
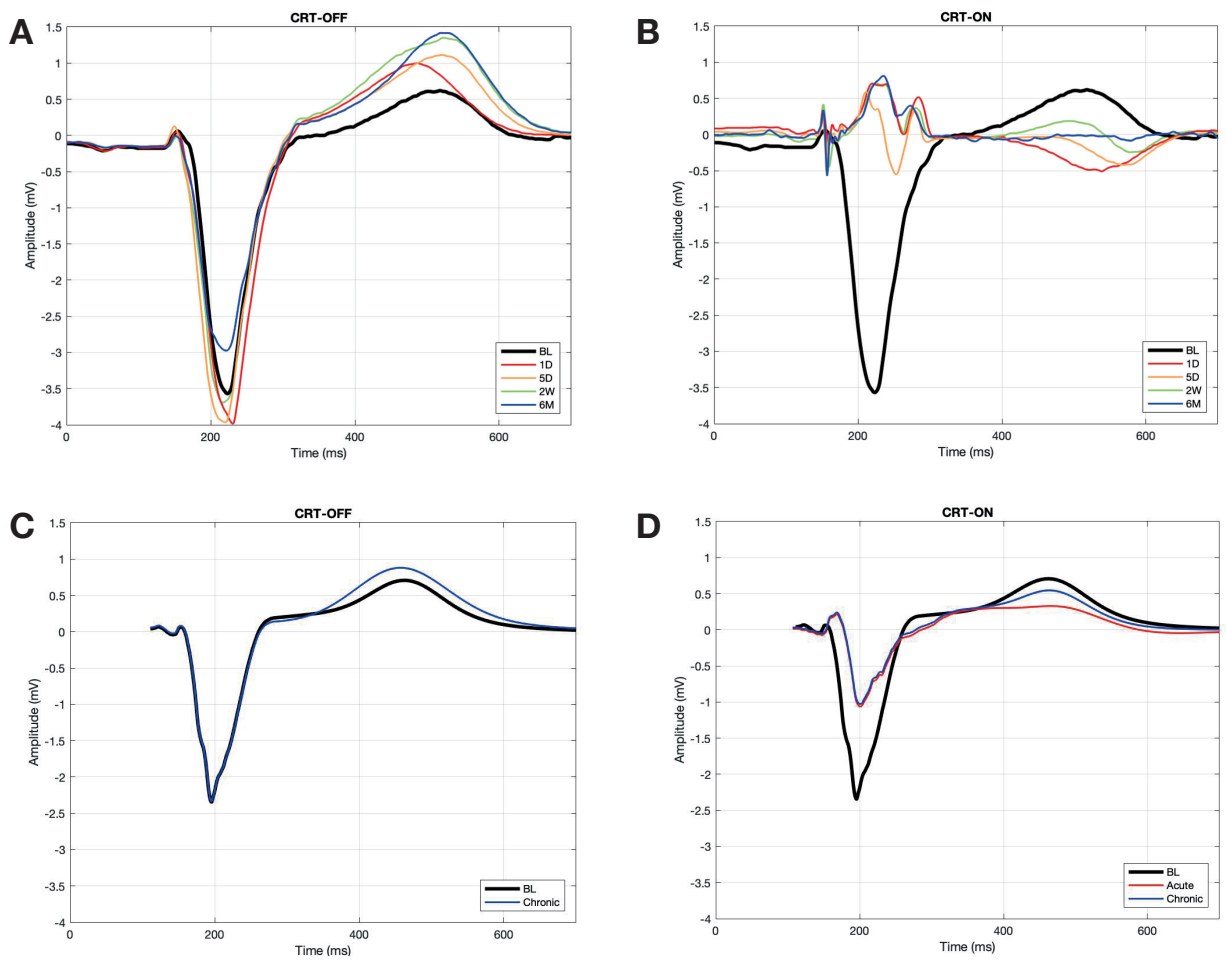

FIGURE 1. Example ECGs (lead V2) during CRT-OFF (A: patient, C: simulation) and CRT-ON (B: patient, D: simulation). Patient ECGs were recorded at heart rates between 59 and 75 beats per minute. ECGs = electrocardiograms, further abbreviations as in Figure 2.

For the whole group of patients CRT caused immediate reductions in QRS area (Figure 2A), amplitude and duration (Table 2), indicating resynchronization of activation. During CRT-OFF, slight but significant increases in QRS area and amplitude were observed between 1 day and 1 month of CRT, although QRS duration did not change (Figure 2A, Table 2). 
A
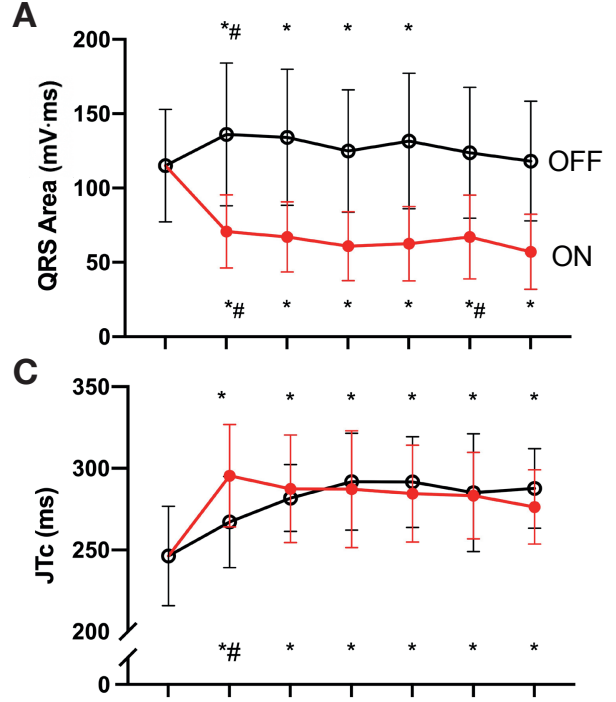

E

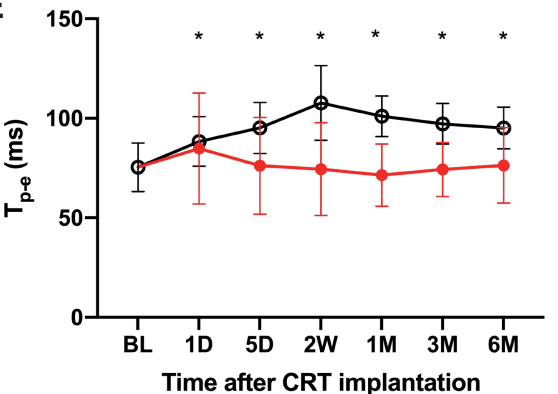

B
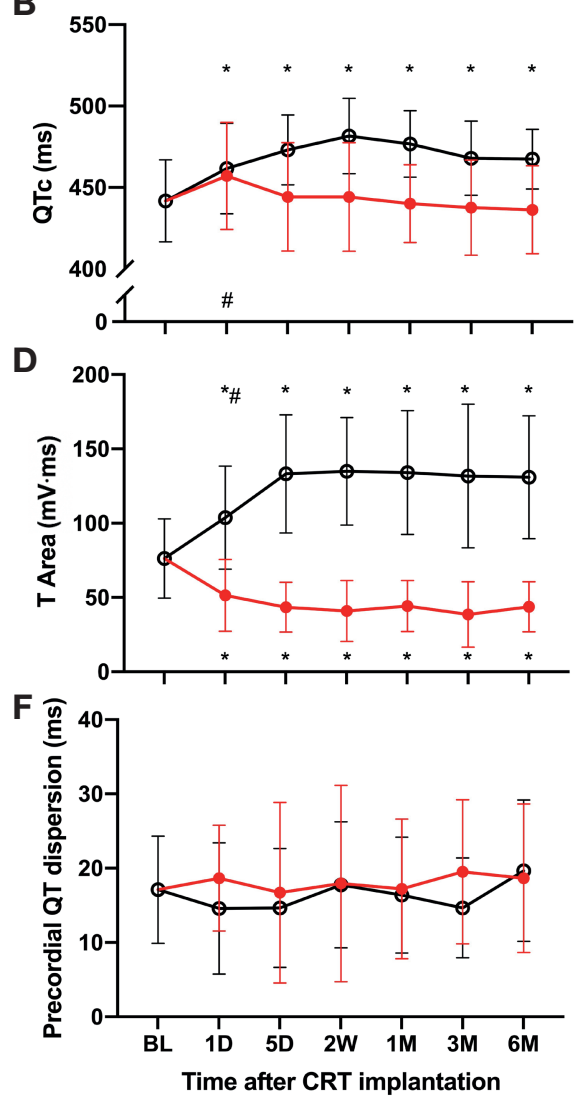

FIGURE 2. Electrocardiographic and vectorcardiographic parameters during CRT-OFF (black symbols) and CRT-ON (red symbols). A. QRS area. B. QTc interval. C. JTc interval. D. T area. E. T p-e. F. Precordial QT dispersion. * $=p \leq 0.05$ vs. $\mathrm{BL} ; \#=\mathrm{p} \leq 0.05$ vs. $6 \mathrm{M}$. $\mathrm{BL}=$ baseline, $1 \mathrm{D}=1$ day, $5 \mathrm{D}=5$ days, $2 \mathrm{~W}=2$ weeks, $1 \mathrm{M}=1$ month, $3 \mathrm{M}=3$ months, $6 \mathrm{M}=6$ months, $\mathrm{CRT}=$ cardiac resynchronization therapy, $\mathrm{T}_{\mathrm{p}-\mathrm{e}}=\mathrm{T}$ peak-to-end interval. 


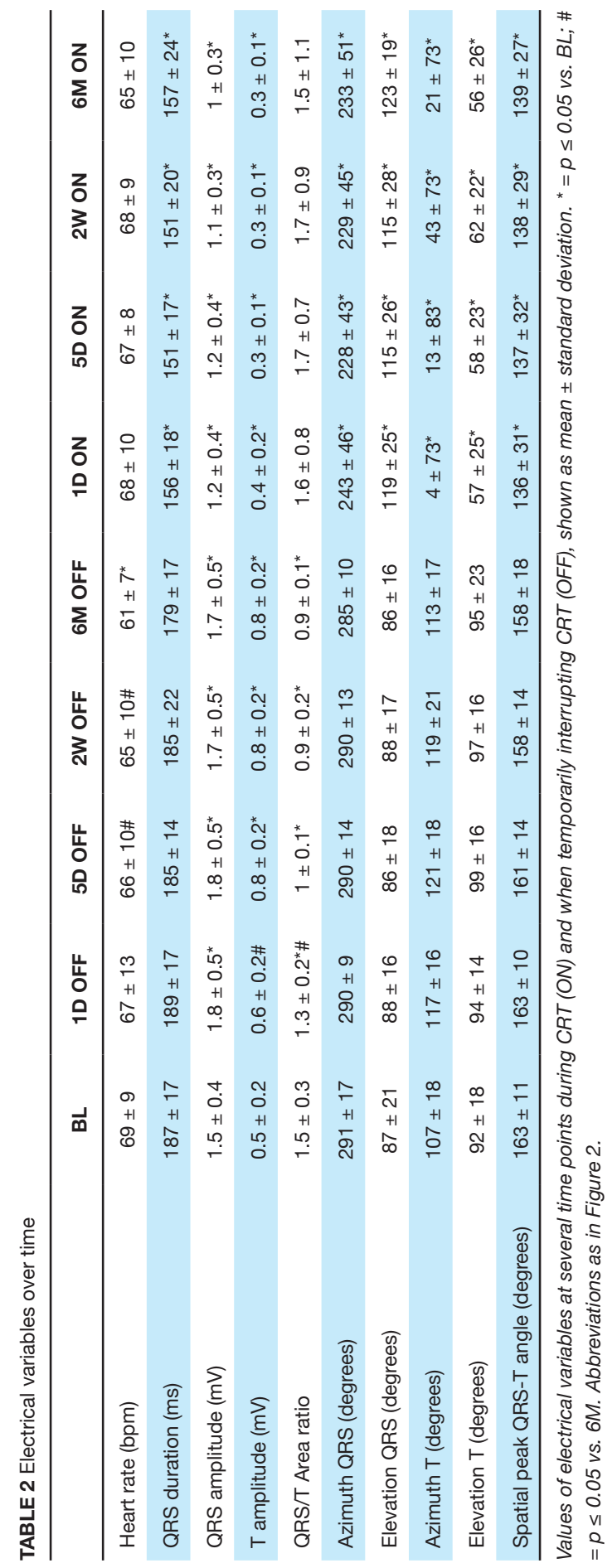


Repolarization was also profoundly affected by CRT. CRT caused a significant $\sim 50 \mathrm{~ms}$ increase in JTc interval, whereas QTc interval only showed a small temporary increase after 1 day of CRT (Figure 2B and C). In contrast, T wave area (Figure 2D) and T wave amplitude (Table 2) decreased during CRT while $T_{p-e}$ and precordial QT dispersion did not show a significant change (Figure 2E and F). While T wave changes during CRT are most likely partly related to altered depolarization patterns during resynchronization, $T$ wave changes during CRT-OFF are entirely due to electrical remodelling. Notably, T area, JTc, QTc and $T_{p-e}$ measured during CRT-OFF all increased within the first two weeks of treatment, while there was no change in precordial QT dispersion (Figure 2, B-F).

Variables describing the direction of the QRS complex and the T wave (elevation and azimuth) changed within 1 day during CRT-ON and remained stable afterwards (Table 2). The spatial angle between QRS and T vectors also showed a quick change during CRT-ON without any further change (Table 2).

\section{Echo parameters}

Immediately upon start of CRT, SRS and IVMD decreased (Figure 3A and B). In contrast, it took approximately two weeks before LVEF, LVESV and SS (Figure 3C-E) showed a significant improvement. LVEF, LVESV and SS remained elevated after temporarily halting CRT, in contrast to IVMD and SRS (CRT-OFF, Figure 3A-E). Eighteen patients (60\% out of 30 with both baseline and CRT-ON echocardiograms) were CRT responders (Table 1).

\section{In silico results}

The procedure to fit the patient-specific model to the baseline QRS complex identified 4 EASs on the RV endocardium, with 2 on the basal-septal region and 2 on the RV free wall. The best fit for the baseline T wave was achieved with an APD90 that was significantly shorter in epicardial and RV endocardial regions than in mid-myocardium and LV endocardium (200 ms vs. 260 ms on average; Figure 4, top panel of middle column). Simulated and recorded baseline ECGs of the patient correlated very well, with Pearson's correlation $r=0.96$. The use of an adaptation factor (see methods) of 0.6 provided the largest similarity between measured and simulated $\mathrm{T}$ area during the four conditions (Table 3). This adaptation factor value also made that the morphology of the simulated ECGs during CRT-ON and CRT-OFF (Figure 1C and D) was quite well comparable to those of the patient (Figure 1A and B). Moreover, simulations and measurements showed minor changes in QT interval (Table 3). 
A
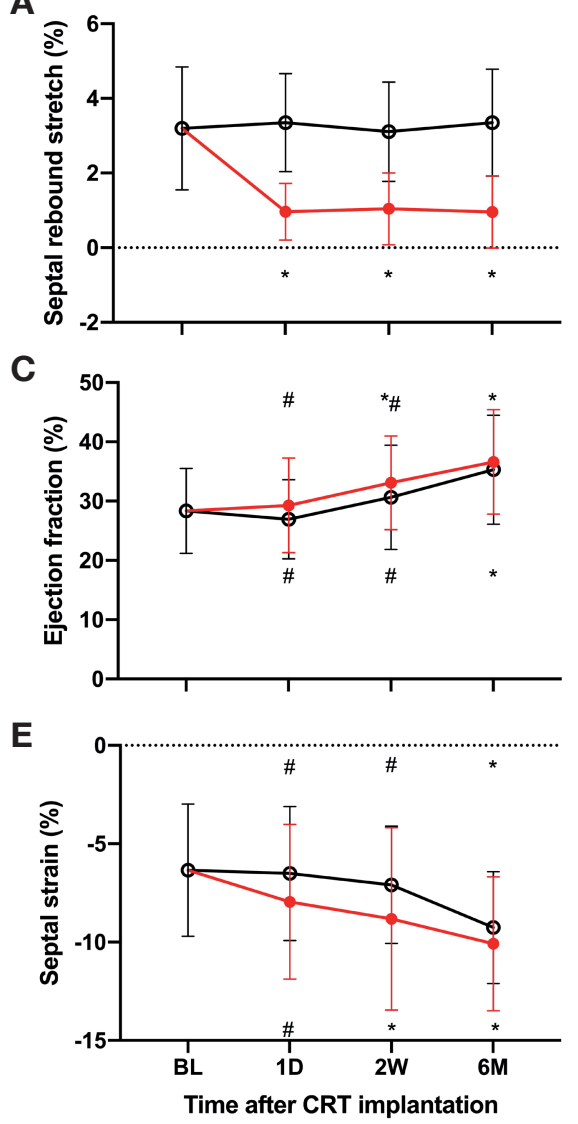

B

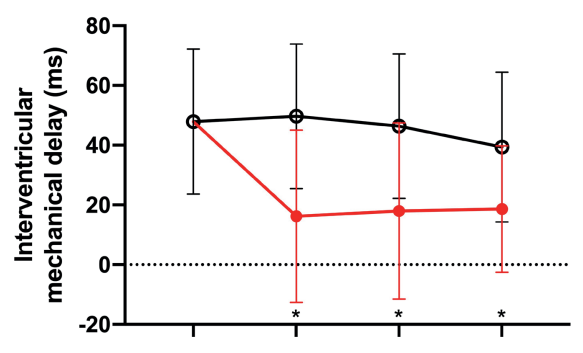

D

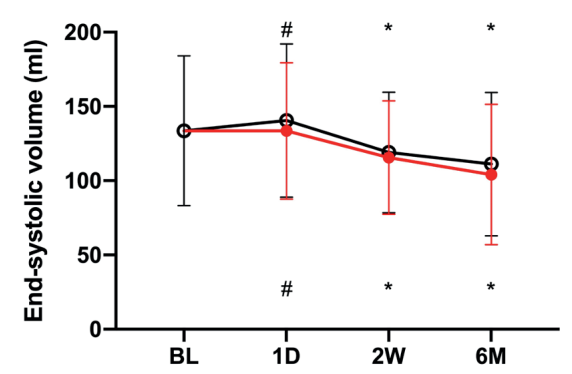

Time after CRT implantation

FIGURE 3. Echocardiographic parameters during CRT-OFF (black symbols) and CRT-ON (red symbols). A. Septal rebound stretch. B. Interventricular mechanical delay. C. Left ventricular ejection fraction. D. Left ventricular endsystolic volume. E. Septal strain. ${ }^{*}=p \leq 0.05$ vs. BL; \# $=p \leq 0.05$ vs. 6 M. Abbreviations as in Figure 2 .

Figure 4 illustrates the activation sequences (left panels), APDs (middle panels) and repolarization sequences (right panels), as calculated from the patient-specific computer model during baseline, acute and chronic CRT-ON and CRT-OFF. Fitting the patient's ECG to the cardiac and thoracic anatomy and ECG electrode positions resulted in a typical left bundle branch block (LBBB)-like sequence of ventricular of activation and earlier RV than LV repolarization (Figure 4, top row). Assuming an adaptation of APD from acute to chronic CRT based on an inverse relation between change in activation time and APD with a slope of 0.6 provided the same relative increase in T area during CRT-OFF as the mean value of the patient cohort (Figure 1, bottom row; Table 3). This adaptation resulted in an increase in APD in the LV lateral wall and in less dispersion between LV free wall segments as well as across the LV free wall (Figure 4 
middle two panels of right column). Overall dispersion of repolarization time (expressed as the $\mathrm{SD}$ of all repolarization times in the simulation) was larger during acute CRT-ON than during chronic CRT-ON (41 vs. 37 ms, Figure 5 and Table 3).

The lower right panel of Figure 4 shows that during chronic CRT-OFF repolarization is considerably delayed in the LV free wall, which was associated with an increase in T wave area derived from the patient-specific simulation (from 76 to $139 \mathrm{mV} \cdot \mathrm{ms}$, Figure $1 \mathrm{C}$ and Table 3). Acutely, switching on CRT reduced both QRS area (from 124 to $15 \mathrm{mV} \cdot \mathrm{ms}$ ) and T area (from 76 to $42 \mathrm{mV} \cdot \mathrm{ms}$, Table 3).

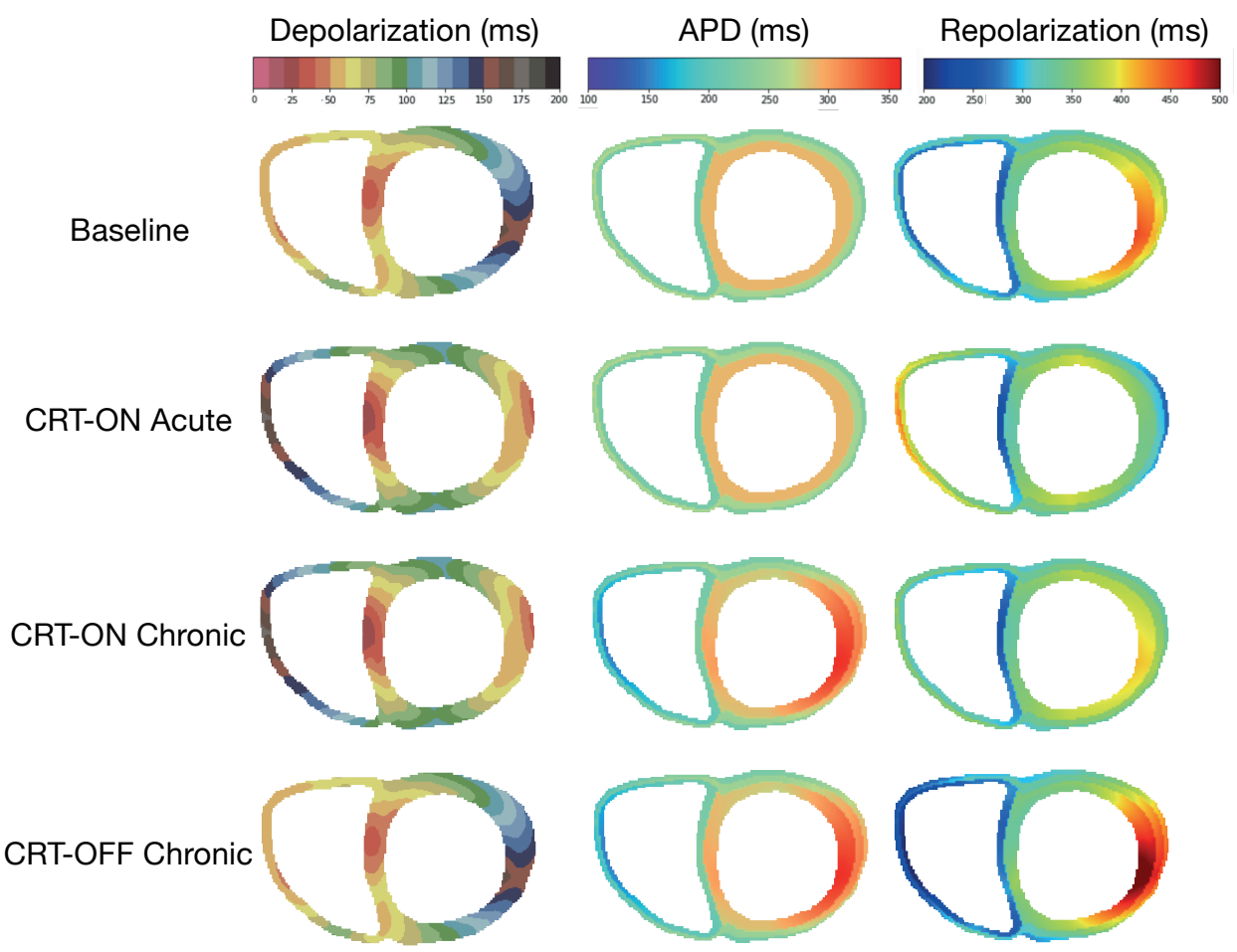

FIGURE 4. Simulations of depolarization times (left column), action potential durations (APD, middle column) and repolarization times (right column), during different situations (row headers). CRT = cardiac resynchronization therapy. 
TABLE 3 Comparison of patient data and simulations

\begin{tabular}{lcccc}
\hline & BL & $\begin{array}{c}\text { ACUTE } \\
\text { CRT-ON }\end{array}$ & $\begin{array}{c}\text { CHRONIC } \\
\text { CRT-ON }\end{array}$ & $\begin{array}{c}\text { CHRONIC } \\
\text { CRT-OFF }\end{array}$ \\
\hline T area $(\mathrm{mV} \cdot \mathrm{ms})$ & $76 \pm 27$ & $51 \pm 24$ & $44 \pm 17$ & $131 \pm 41$ \\
\hline area $(\mathrm{mV} \cdot \mathrm{ms})$ & 76 & 42 & 49 & 139 \\
\hline QT interval $(\mathrm{ms})$ & $423 \pm 26$ & $441 \pm 45$ & $428 \pm 35$ & $467 \pm 24$ \\
\hline QT interval $(\mathrm{ms})$ & 525 & 547 & 519 & 535 \\
\hline SD of repolarization times $(\mathrm{ms})$ & 46 & 41 & 37 & 69 \\
\hline
\end{tabular}

Values of $T$ area and $Q T$ interval for patients (mean $\pm S D$ ) and, in italics, those obtained from simulations. $B L=$ baseline, $C R T=$ cardiac resynchronization therapy, $S D=$ standard deviation.
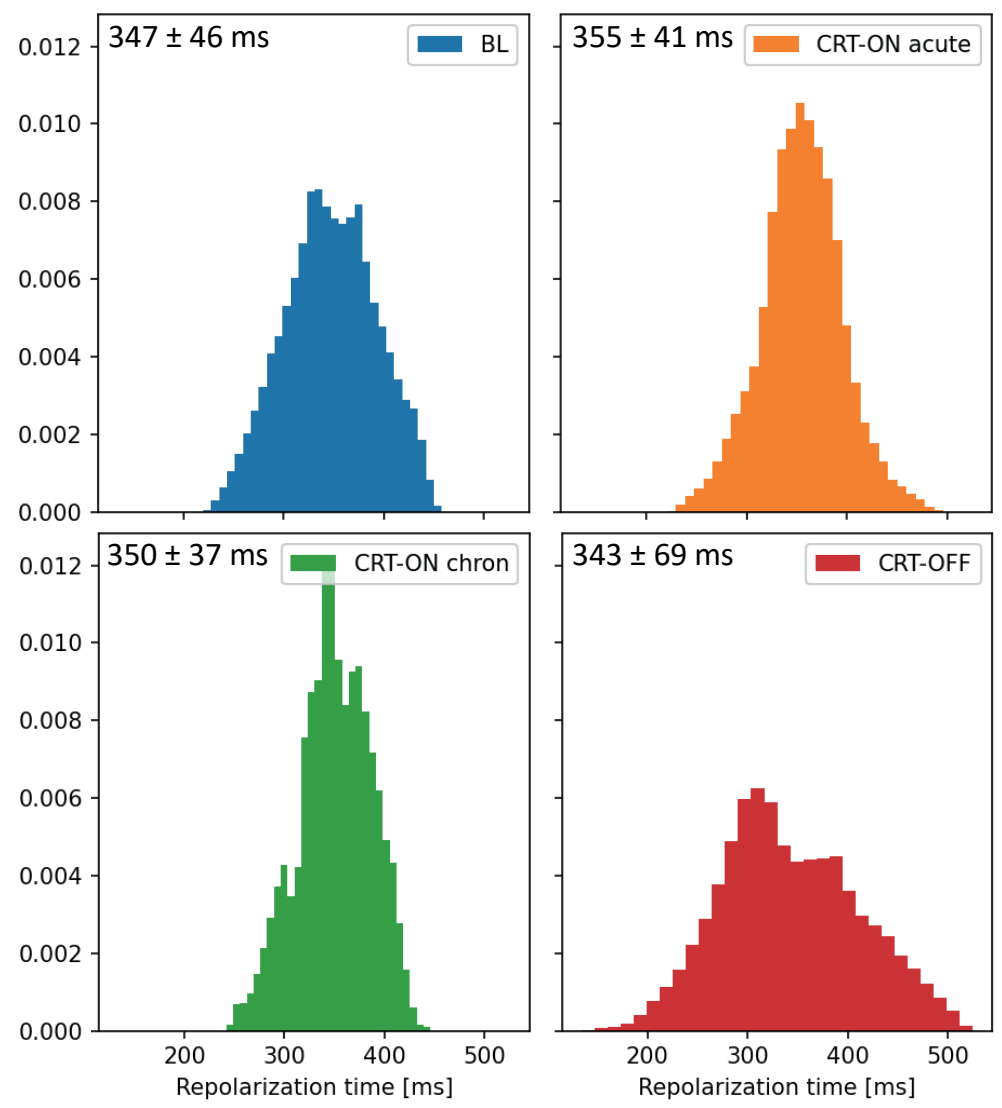

FIGURE 5. Distribution of repolarization times during the different simulations. Mean \pm standard deviation of repolarization times $(\mathrm{ms})$ is indicated. $\mathrm{BL}=$ baseline, $\mathrm{CRT}=$ cardiac resynchronization therapy. 


\section{Discussion}

The main findings of the patient study are that 1) during CRT-ON, electrocardiographic repolarization parameters yield conflicting results and that 2) electrocardiographic measurements during transient CRT-OFF reveal signs of repolarization remodelling within two weeks of CRT, which precede mechanical remodelling. The patient-specific computer simulation indicates that the $T$ wave changes during temporary interruption of chronic CRT may be explained by electrical remodelling processes that lead to a reduction in dispersion of repolarization during chronic CRT.

\section{Assessing repolarization changes using a patient-specific computer simulation}

A special and important feature of this study is the patient-specific computer simulation. The way the depolarization pattern and conduction properties of the patient were acquired from the 12-lead ECG and cardiac MRI has been described and validated previously ${ }^{19,20}$. The repolarization sequence was calculated using the same approach. The activation pattern during CRT was calculated using the estimated conduction properties and cardiac geometry, while choosing locations of the RV and LV leads that are frequently used. Finally, for assessing changes in repolarization during chronic CRT we assumed an inverse relation between (change in) activation time and APD, as demonstrated in rabbit hearts ${ }^{22}$. Such a relation has also been found in three rejected isolated perfused human donor hearts ${ }^{23}$ and in patients with LBBB prior to $\mathrm{CRT}^{24}$. The factor of 0.6 of this inverse relation was based on the fact that this factor provided the best match in $T$ area changes seen in simulations and patient data for the four conditions studied. Note that T area and QT interval showed somewhat different behaviour, suggesting that the model provides a fairly unique solution.

\section{Remodelling of repolarization}

The present study demonstrates that sequential T wave measurements during CRT-OFF reveal changes in repolarization after 5-14 days of CRT that are not observed when measuring the T wave during CRT-ON. This observation may be explained by the fact that the CRT-induced bifocal spread of activation dominates the repolarization sequence and conceals regional repolarization changes. Adaptations in repolarization during longer-lasting CRT are in line with previous publications describing the occurrence of such changes when patients develop LBBB or LBBB-like activation, such as induced by RV pacing. Under those conditions the T wave is large immediately upon initiation of pacing or developing LBBB while it becomes smaller during subsequent days to weeks ${ }^{25,26}$, a time span similar to the one observed in our CRT study. 
The T wave changes during CRT-OFF are reminiscent of the phenomenon of cardiac memory, which is observed when returning to normal activation after a period of ventricular pacing. When pacing is switched off, the T wave remains abnormal despite the return of the normal QRS complex. In the context of CRT, interruption of pacing lets the heart return to the more abnormally activated baseline state (i.e. LBBB). Actually, the presence of larger T waves upon interrupting CRT after longer duration of treatment is quite similar to the inverse process observed after sudden onset of $\operatorname{LBBB}^{26,27}$.

From studies on cardiac memory it is known that the $T$ wave changes occur by regional changes in $\mathrm{APD}$, prolonging in early-activated regions and shortening in regions of late activation during the dominant activation sequence ${ }^{22,28}$. The model simulations presented in this study indicate that this way of adaptation may also be present in CRT patients. Importantly, this way of adaptation leads to a reduction in dispersion of repolarization especially in the LV wall.

The present finding that APD in the LV lateral wall increases after starting CRT may seem in contradiction to the findings of Chen et al. ${ }^{29}$. These investigators reported that CRT shortened the activation recovery interval (ARI, a surrogate measure of APD) measured within $\sim 1 \mathrm{~cm}$ from the LV pacing site, in particular in patients responding to the therapy. However, these changes occurred considerably slower (between 6 weeks and 6 months of CRT) than the increase in $T$ area in the present study (within 5 days and remaining constant afterwards). Therefore, we think that the ARI shortening represents a more local and slower process. Of note, the ARI shortening occurred in the same time frame as the increase in LVEF in both the Chen and the present study. It is possible that the negative impact of heart failure on cardiac tissue function is mitigated faster near the LV pacing site, because the load of that region is most pronouncedly reduced due to its early activation during CRT. Therefore, the ARI reduction may represent a decrease in APD due to decreasing severity of heart failure. In contrast, the increase in T area we found within 5 days of starting CRT may be caused by mechano-electrical coupling. T area, being a more global measure of repolarization, may miss the local APD changes as measured by a local electrogram by Chen et al.

The present study does not allow speculation about the molecular mechanisms involved in repolarization remodelling, but it suffices to refer to the extensive work of the group of Dr. Michael Rosen, who showed involvement of stretch-induced local release of angiotensin II, modulation of transcription factors and changes in $\mathrm{I}_{\mathrm{Kr}}$ and $\mathrm{I}_{\mathrm{Ca}, \mathrm{L}}{ }^{11}$. Regional differences in stretch, coinciding with abnormal activation, were shown to be a crucial factor for inducing cardiac memory ${ }^{30}$. Experimentally, Sosunov et al. showed that reduction of cardiac mechanics by unloading the LV or blocking excitation-contraction coupling attenuated the development of cardiac memory ${ }^{30}$. Based on this information Kuijpers et al. proposed that $\mathrm{T}$ wave memory is mediated by mechano-electrical feedback, aiming at homogenization of myocardial work. 
Using computer simulations they found that such mechano-electrical coupling leads to a prolonged APD near the LV pacing region and a lower overall dispersion of repolarization ${ }^{12}$, which is in line with the results of the present study.

\section{T wave changes during CRT}

The present study indicates that the apparently contradicting changes in T area and JTc during CRT may be explained by the different aspects of repolarization measured by these parameters. JTc reflects the mean duration of the ventricular action potential, after all it is calculated as the difference between QT and QRS interval. T area, on the other hand, presumably increases with longer JTc as well as with a more unidirectional repolarization (i.e. less cancellation of repolarization forces).

The three-dimensional simulation data illustrate that during acute CRT-ON there is more cancellation of repolarization than during LBBB, especially in the LV (fitting with a reduction in $T$ area), whereas the difference between earliest and latest repolarized region does not decrease (fitting with a small increase in QTc, observed in the patients). The histograms of repolarization times in the simulations (Figure 5) clarify these ideas by showing a decrease in SD of repolarization times but an increase in the time between earliest and latest repolarization during acute CRT-ON compared to baseline.

Clearly, all ECG and VCG measures of dispersion of repolarization describe only part of the total dispersion. $T$ wave area is an integral measure, presumably describing mainly overall dispersion of repolarization ${ }^{31}$. As mentioned above, QTC and JTc primarily indicate the maximum repolarization time ${ }^{2}$. Precordial QT dispersion has been suggested to provide a more detailed impression of the dispersion of repolarization, although $T$ vector orientation relative to the different leads has been proposed as the underlying mechanism of this phenomenon, too $^{32}$. $T_{p-e}$ has been announced as a marker of transmural dispersion of repolarization, although there is also considerable dispute about this idea ${ }^{3,4,33}$. To complicate the picture even more, epicardial LV pacing can be expected to increase the transmural dispersion of repolarization directly underneath the pacing electrode, but this difference may be small in regions more remote from the pacing site 4 . To summarize, ECG and VCG measures of repolarization each provide a limited impression of this process. The present study does indicate that the clearest impression of changes in repolarization in CRT patients can be obtained by briefly interrupting CRT.

\section{Remodelling in activation?}

Interestingly, in our study, QRS area and QRS amplitude increased slightly (by 15\%) but significantly during CRT-OFF during the first month of CRT. The data in the present study do 
not provide a direct explanation of this unexpected finding. However, several animal studies have demonstrated that dyssynchrony can cause abnormal distribution of connexin 43 (Cx43), including lower expression levels and altered subcellular localization in the lateral wall of the $\mathrm{LV}^{34,35}$. Cx43 relocalization has also been observed after stretching cultured cardiomyocytes ${ }^{36}$. Since dyssynchrony causes major local mechanical changes, these findings together support the idea that dyssynchrony-induced mechanical derangements may affect myocardial impulse conduction in the heart. Since cellular uncoupling may lead to lower QRS amplitudes ${ }^{37}$, it may be speculated that an increase in Cx43 expression and/or more polarized Cx43 localization may lead to more uniform (but not overall faster) depolarization, resulting in the increase in QRS area after CRT. However, further studies are needed to corroborate these findings and to better understand this (transient) phenomenon.

\section{Limitations}

This study has several limitations. First of all, the number of patients that was studied was small. Secondly, the observational nature of the study implied that patient treatment was not protocolled but was adjusted as required, and e.g. device settings were changed when needed. ECG measurements were obtained at more time points than echocardiographic measurements. However, the two-week measurements were performed for both variables and LVEF and SS continued to increase towards 6 months, whereas repolarization markers levelled off at two weeks.

\section{Future directions}

The present study describes the $T$ wave changes after various durations of CRT. The small study size precluded investigating the relation of these $T$ wave changes with arrhythmia risk. The use of ECG and VCG provided only relatively global information on cardiac activation and repolarization. Detailed cardiac mapping (e.g. non-invasive non-contact (ECG imaging) or invasive contact mapping) may provide more in-depth information about the regional repolarization changes after CRT and may solve the question which of the markers of dispersion of repolarization may be most relevant for predicting arrhythmias.

\section{Conclusions}

CRT causes differential changes in various T wave markers that occur relatively fast ( $<2$ weeks) after onset of CRT. These changes precede mechanical remodelling and indicate a reduction in dispersion of repolarization during longer-lasting CRT. Our electrocardiographic findings are 
corroborated by computer modelling, using a patient-specific model. Lengthening of APDs in zones of early activation and shortening of APDs in zones of late activation in the model yielded $\mathrm{T}$ area changes that were quite similar to those observed in patients.

\section{Acknowledgements}

This study was supported by the Swedish Heart-Lung Foundation and the Swedish Society of Medicine. 


\section{References}

1. Brignole M, Auricchio A, Baron-Esquivias G, Bordachar P, Boriani G, Breithardt O-A, et al. 2013 ESC Guidelines on cardiac pacing and cardiac resynchronization therapy. Eur Heart J 2013;34:2281329.

2. Medina-Ravell VA, Lankipalli RS, Yan G-X, Antzelevitch C, Medina-Malpica NA, Medina-Malpica $\mathrm{OA}$, et al. Effect of epicardial or biventricular pacing to prolong QT interval and increase transmural dispersion of repolarization: does resynchronization therapy pose a risk for patients predisposed to long QT or torsade de pointes? Circulation 2003;107:740-6.

3. Fish JM, Diego JM Di, Nesterenko V, Antzelevitch C. Epicardial Activation of Left Ventricular Wall Prolongs QT Interval and Transmural Dispersion of Repolarization: Implications for Biventricular Pacing. Circulation 2004;109:2136-42.

4. Hooft van Huysduynen B, Swenne CA, Bax JJ, Bleeker GB, Draisma HHM, Erven L van, et al. Dispersion of repolarization in cardiac resynchronization therapy. Hear Rhythm 2005;2:1286-93.

5. Thijssen J, Borleffs CJW, Delgado V, Rees JB Van, Mooyaart EAQ, Bommel RJ Van, et al. Implantable cardioverter-defibrillator patients who are upgraded and respond to cardiac resynchronization therapy have less ventricular arrhythmias compared with nonresponders. J Am Coll Cardiol 2011;58:2282-9.

6. Lin G, Rea RF, Hammill SC, Hayes DL, Brady PA. Effect of cardiac resynchronisation therapy on occurrence of ventricular arrhythmia in patients with implantable cardioverter defibrillators undergoing upgrade to cardiac resynchronisation therapy devices. Heart 2008;94:186-90.

7. Gold MR, Linde C, Abraham WT, Gardiwal A, Daubert JC. The impact of cardiac resynchronization therapy on the incidence of ventricular arrhythmias in mild heart failure. Hear Rhythm 2011;8:67984.

8. Barsheshet A, Wang PJ, Moss AJ, Solomon SD, Al-Ahmad A, McNitt S, et al. Reverse remodeling and the risk of ventricular tachyarrhythmias in the MADIT-CRT (Multicenter Automatic Defibrillator Implantation Trial-Cardiac Resynchronization Therapy). J Am Coll Cardiol 2011;57:2416-23.

9. Itoh M, Yoshida A, Fukuzawa K, Kiuchi K, Imamura K, Fujiwara R, et al. Time-dependent effect of cardiac resynchronization therapy on ventricular repolarization and ventricular arrhythmias. Europace 2013;15:1798-804.

10. Wecke L, Deursen CJM Van, Bergfeldt L, Prinzen FW. Repolarization changes in patients with heart failure receiving cardiac resynchronization therapy - Signs of cardiac memory. J Electrocardiol 2011;44:590-8.

11. Rosen MR, Bergfeldt L. Cardiac memory: The slippery slope twixt normalcy and pathology. Trends Cardiovasc Med 2015;25:687-96.

12. Kuijpers NHL, Hermeling E, Lumens J, Eikelder HMM ten, Delhaas T, Prinzen FW. Mechano-electrical coupling as framework for understanding functional remodeling during LBBB and CRT. Am J Physiol - Hear Circ Physiol 2014;306:H1644-59.

13. Padeletti L, Fantappiè C, Perrotta L, Ricciardi G, Pieragnoli P, Chiostri M, et al. Cardiac memory in humans: Vectocardiographic quantification in cardiac resynchronization therapy. Clin Res Cardiol 2011;100:51-6. 
14. Engels EB, Alshehri S, Deursen CJM Van, Wecke L, Bergfeldt L, Vernooy K, et al. The synthesized vectorcardiogram resembles the measured vectorcardiogram in patients with dyssynchronous heart failure. J Electrocardiol 2015;48:586-92.

15. Hermans BJM, Vink AS, Bennis FC, Filippini LH, Meijborg VMF, Wilde AAM, et al. The development and validation of an easy to use automatic QT-interval algorithm. PLOS One 2017;12:e0184352.

16. Vandenberk B, Vandael E, Robyns T, Vandenberghe J, Garweg C, Foulon V, et al. Which QT Correction Formulae to Use for QT Monitoring? J Am Heart Assoc 2016;5:e003264.

17. Everdingen WM van, Schipper JC, 'T Sant J Van, Misier KR, Meine M, Cramer MJ. Echocardiography and cardiac resynchronisation therapy, friends or foes? Netherlands Hear J 2016;24:25-38.

18. Leenders GE, Boeck BWL De, Teske AJ, Meine M, Bogaard MD, Prinzen FW, et al. Septal rebound stretch is a strong predictor of outcome after cardiac resynchronization therapy. J Card Fail 2012;18:404-12.

19. Pezzuto S, Kal'avský P, Potse M, Prinzen FW, Auricchio A, Krause R. Evaluation of a rapid anisotropic model for ECG simulation. Front Physiol 2017;8:art. 265.

20. Pezzuto S, Prinzen FW, Potse M, Maffessanti F, Regoli F, Caputo ML, et al. Reconstruction of threedimensional biventricular activation based on the 12-lead electrocardiogram via patient-specific modelling. Europace 2020; advance online publication, doi:10.1093/europace/euaa330.

21. Potse M. Scalable and accurate ECG simulation for reaction-diffusion models of the human heart. Front Physiol 2018;9:art. 370.

22. Costard-Jäckle A, Goetsch B, Antz M, Franz MR. Slow and long-lasting modulation of myocardial repolarization produced by ectopic activation in isolated rabbit hearts. Evidence for cardiac 'memory'. Circulation 1989;80:1412-20.

23. Opthof T, Remme CA, Jorge E, Noriega F, Wiegerinck RF, Tasiam A, et al. Cardiac activationrepolarization patterns and ion channel expression mapping in intact isolated normal human hearts. Hear Rhythm 2017;14:265-72.

24. Maffessanti F, Wanten J, Potse M, Regoli F, Caputo ML, Conte G, et al. The relation between local repolarization and T-wave morphology in heart failure patients. Int J Cardiol 2017;241:270-6.

25. Shvilkin A, Bojovic B, Vajdic B, Gussak I, Zimetbaum P, Josephson ME. Vectorcardiographic determinants of cardiac memory during normal ventricular activation and continuous ventricular pacing. Hear Rhythm 2009;6:943-8.

26. Engels EB, Poels TT, Houthuizen P, Jaegere PPT de, Maessen JG, Vernooy K, et al. Electrical remodelling in patients with iatrogenic left bundle branch block. Europace 2016;18:iv44-52.

27. Shvilkin A, Bojovic B, Vajdic B, Gussak I, Ho KK, Zimetbaum P, et al. Vectorcardiographic and electrocardiographic criteria to distinguish new and old left bundle branch block. Hear Rhythm 2010;7:1085-92.

28. Marrus SB, Andrews CM, Cooper DH, Faddis MN, Rudy Y. Repolarization Changes Underlying LongTerm Cardiac Memory Due to Right Ventricular Pacing. Circ Arrhythmia Electrophysiol 2012;5:77381.

29. Chen Z, Hanson B, Sohal M, Sammut E, Child N, Shetty A, et al. Left ventricular epicardial electrograms show divergent changes in action potential duration in responders and nonresponders to cardiac resynchronization therapy. Circ Arrhythmia Electrophysiol 2013;6:265-71. 
30. Sosunov EA, Anyukhovsky EP, Rosen MR. Altered ventricular stretch contributes to initiation of cardiac memory. Hear Rhythm 2008;5:106-13.

31. Huysduynen BH Van, Swenne CA, Draisma HHM, Antoni ML, Vooren H Van De, Wall EE Van Der, et al. Validation of ECG indices of ventricular repolarization heterogeneity: A computer simulation study. J Cardiovasc Electrophysiol 2005;16:1097-103.

32. Lee KW, Kligfield P, Okin PM, Dower GE. Determinants of precordial QT dispersion in normal subjects. J Electrocardiol 1998;31:128-33.

33. Kors JA, Ritsema van Eck HJ, Herpen G van. The meaning of the Tp-Te interval and its diagnostic value. J Electrocardiol 2008;41:575-80.

34. Spragg DD, Akar FG, Helm RH, Tunin RS, Tomaselli GF, Kass DA. Abnormal conduction and repolarization in late-activated myocardium of dyssynchronously contracting hearts. Cardiovasc Res 2005;67:77-86.

35. Spragg DD, Leclercq C, Loghmani M, Faris OP, Tunin RS, DiSilvestre D, et al. Regional alterations in protein expression in the dyssynchronous failing heart. Circulation 2003;108:929-32.

36. Salameh A, Wustmann A, Karl S, Blanke K, Apel D, Rojas-Gomez D, et al. Cyclic mechanical stretch induces cardiomyocyte orientation and polarization of the gap junction protein connexin43. Circ Res 2010;106:1592-602.

37. Potse M, Krause D, Bacharova L, Krause R, Prinzen FW, Auricchio A. Similarities and differences between electrocardiogram signs of left bundle-branch block and left-ventricular uncoupling. Europace 2012;14:v33-9. 


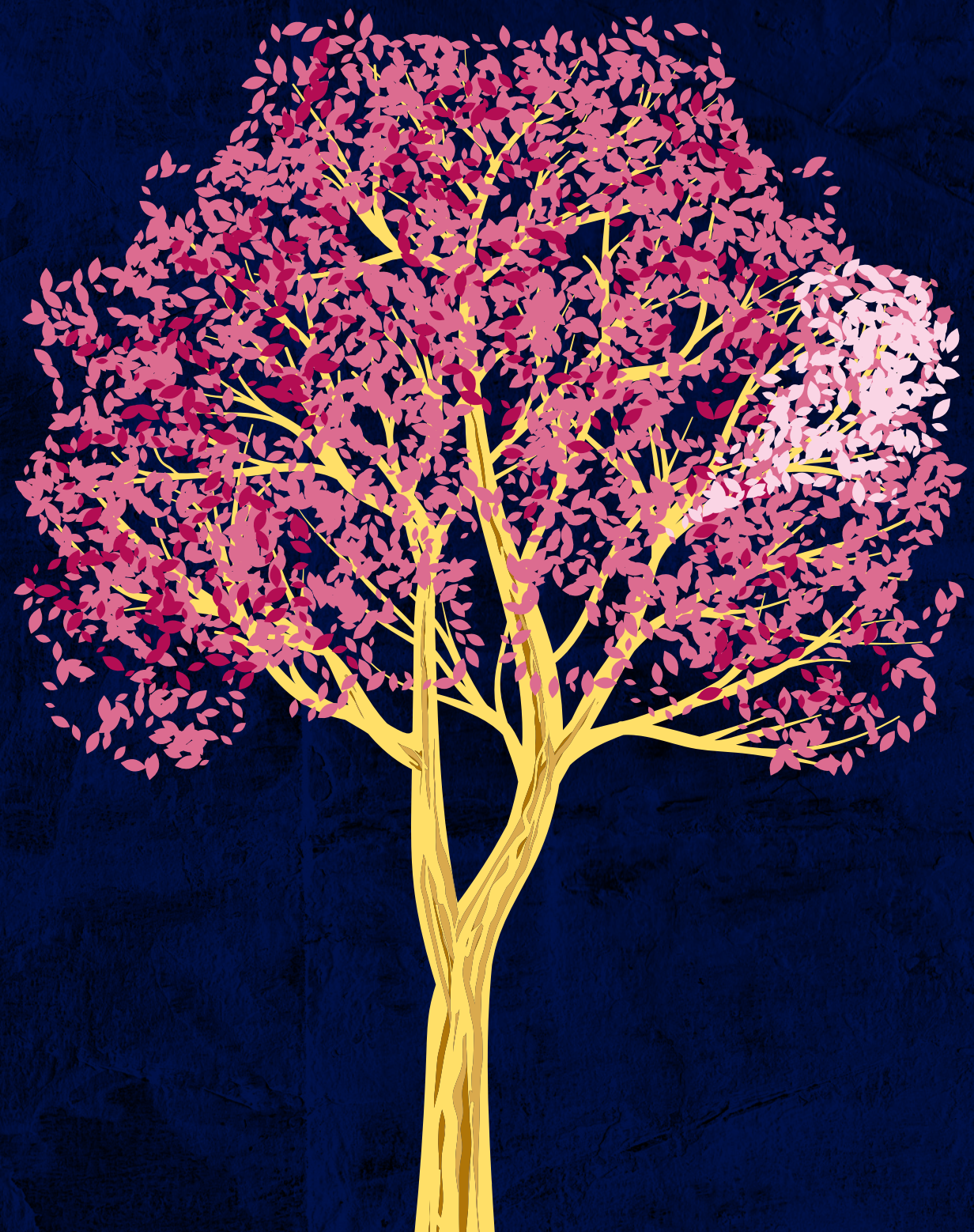




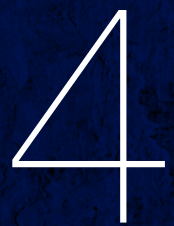

\section{Relation between repolarization remodelling and response to cardiac resynchronization therapy}

Nienke J. Verzaal ${ }^{1 \star}$, Caroline J.M. van Deursen ${ }^{2 \star}$, Liliane Wecke $^{3}$, Renée Boereboom", Kevin Vernooy ${ }^{2}$, Tammo Delhaas ${ }^{4}$ and Frits W. Prinzen 1

\footnotetext{
'Department of Physiology, Maastricht University, Maastricht, The Netherlands

${ }^{2}$ Department of Cardiology, Maastricht University Medical Centre, Maastricht, The Netherlands

${ }^{3}$ Heart Clinic, Capio St. Göran's Hospital, Stockholm, Sweden

${ }^{4}$ Department of Biomedical Engineering, Maastricht University, Maastricht, The Netherlands

* These authors contributed equally.
} 


\section{Abstract}

Background The beneficial effects of cardiac resynchronization therapy (CRT) on cardiac function are well established. However, less is known about the connection between contractile and electrical remodelling. Previously we showed that the change in $\mathrm{T}$ area after temporarily interrupting CRT is an indicator of electrical remodelling of repolarization. The aim of this study is to investigate the link between T wave changes and 1) echocardiographic response following CRT and 2) occurrence of arrhythmias following CRT.

Methods In 76 patients, electrocardiograms (ECGs) were recorded prior to CRT implantation (baseline, BL) and after 6-12 months of treatment (both during CRT (CRT-ON) and during brief interruption of CRT (CRT-OFF)). Vectorcardiograms (VCGs) were synthesized from the 12-lead ECGs. JTC and QTC intervals, T peak-to-end interval $\left(T_{p-e}\right)$, precordial QT dispersion and VCG-derived T area were used as measures of repolarization. Echocardiograms, 24-hour Holter recordings (at BL and 6 months) and device readout data on arrhythmias (2 weeks and 6 months after implantation) were collected.

Results The cohort was divided into subgroups with a T area change smaller (LOW) and larger $(\mathrm{HIGH})$ than the median T area change measured during CRT-OFF. There were no significant differences in baseline QRS duration, QRS area or T area between these subgroups. Patients in the HIGH subgroup showed a larger increase in left ventricular ejection fraction than those in the LOW subgroup ( $11 \pm 8$ vs. $6 \pm 6 \%$ respectively, $p \leq 0.05)$. QTc, JTc, $T_{p-e}$ and T wave vector angles were not significantly different between the subgroups. Arrhythmia incidence was low and did not differ between the subgroups.

Conclusions Repolarization remodelling, as indicated by a large $T$ area change during CRTOFF at follow-up, is associated with better echocardiographically measured response to CRT. Consequences of these $T$ area changes for arrhythmia incidence need to be investigated in larger studies. 


\section{Introduction}

Cardiac resynchronization therapy (CRT) is an effective treatment for dyssynchronous heart failure, improving cardiac pump function as well as survival ${ }^{1}$. A short-term study in patients, however, suggests a potential pro-arrhythmic effect of CRT through increased dispersion of repolarization². A similar mechanism was also observed in animal tissues during epicardial pacing ${ }^{3}$. On the other hand, several other studies in patients, including clinical trials, demonstrate that arrhythmia incidence following CRT may decrease in responders, while it may increase in non-responders ${ }^{4,5}$. The difference in arrhythmia risk between responders and non-responders suggests a link between electrical and mechanical remodelling following CRT.

We have previously investigated electrical remodelling following $\mathrm{CRT}^{6}$, in particular changes in repolarization dispersion (chapter 3). In those studies, we demonstrated that changes in T wave area when the CRT device is briefly switched off (indicative of repolarization remodelling) already occur within the first days to weeks of treatment. The reason that $T$ wave area may be a good method to study repolarization remodelling is that it depends on both the duration and homogeneity of repolarization?

Computer modelling data from the previous study (chapter $\mathbf{3}$ ) indicated that repolarization remodelling reduces the inhomogeneity of repolarization. For this reason, and because of the link between electrical and mechanical remodelling described earlier, we hypothesize that extensive repolarization remodelling is associated with a larger improvement in left ventricular ejection fraction (LVEF) and fewer arrhythmias.

The aim of the present study is to explore the potential link between repolarization remodelling and 1) LVEF change and 2) ventricular arrhythmia incidence after CRT. To this end, T area and other electrocardiographic (ECG) and vectorcardiographic (VCG) parameters were assessed in patients, prior to device implantation and after 6-12 months of CRT. At followup, measurements were performed both while CRT was on (CRT-ON) and when CRT was briefly switched off (CRT-OFF). Additionally, information about arrhythmias (24-hour Holter recordings, device readouts) and CRT response (echocardiography) was obtained. Patients were stratified according to $T$ area change (indicative of the amount of electrical remodelling) between baseline and the CRT-OFF measurements, and parameters were compared between those who had a small T area change (LOW) and those who had a large T area change (HIGH). 


\section{Materials and methods}

\section{Patients}

This study included consecutive patients, referred to the Maastricht University Medical Centre for possible CRT implantation. Patients who were (1) 18 years of age or older; (2) had an indication for CRT; (3) did not have any known condition that could limit life expectancy to less than 6 months as of referral and (4) were capable of giving informed consent were asked to participate. The study conforms to the Declaration of Helsinki. The protocol was approved by the ethics committee of Maastricht University Medical Centre (project number 10-2-090) and all patients gave written informed consent to participate before inclusion.

\section{Study protocol}

Baseline 12-lead ECGs were obtained within one year prior to implantation of a CRT device. Follow-up ECGs were recorded 6 (range: 3-13) months after implantation, both during CRTON and while the CRT device was briefly switched off (CRT-OFF). Echocardiography was performed before implantation and 6 (5-10) months after implantation. Holter recordings were obtained both prior to starting CRT and after 6 (5-8) months. CRT device readouts were performed after 2 (1-3) weeks and 6 (4-9) months of therapy.

\section{VCG analysis}

ECGs were extracted from the MUSE Cardiology Information system (GE Healthcare, Chicago, US). Using custom-written Matlab software, ECGs were transformed to VCGs utilizing the Kors matrix and semi-automatic analysis was performed as described previously ${ }^{8}$. Maximum QRS and T vectors were defined as the maximum distances between the origin of the VCG loop and a point on the 3D QRS and T loops, respectively. The size and direction of the maximum vectors were expressed by the vector amplitude, azimuth (angle in the transverse plane, 0 is at the left arm; for QRS azimuth, the values were expressed on a full circle $\left(360^{\circ}\right)$ ) and elevation (angle in the craniocaudal plane). Furthermore, the area under the curve of the QRS complex and T wave in the X, Y, and Z direction was determined by numerical integration from the beginning to the end of the QRS complex or T wave, respectively (Figure 3, General introduction). Subsequently, the total areas of the QRS and T loops were determined using these formulas:

$$
Q R S_{\text {area }}=\sqrt{Q R S_{\text {area }, x}^{2}+\mathrm{QRS}_{\text {area }, y}^{2}+\mathrm{QRS}_{\text {area }, \mathrm{z}}^{2}} \text { and } T_{\text {area }}=\sqrt{T_{\text {area }, \mathrm{x}}^{2}+\mathrm{T}_{\text {area }, y}^{2}+\mathrm{T}_{\text {area }, \mathrm{z}}^{2}}
$$

The different effects of CRT on depolarization and repolarization were further explored by calculating the QRS-T area ratio. 
QT interval was measured automatically using the tangent method as described previously ${ }^{9}$ and subsequently corrected for heart rate (QTC) using Fridericia's formula, since this formula has been shown to be superior to Bazett's formula10. Median T peak-to-end interval $\left(T_{p-e}\right)$ for all leads as well as precordial QT dispersion (standard deviation of start Q to precordial T ends) were determined. The JTc interval was determined by subtracting QRS duration from the QT interval and subsequently applying Fridericia's formula.

\section{Echocardiography}

Echocardiograms were obtained using an iE33 system (Philips Medical Systems, Best, the Netherlands). Left ventricular end-systolic and diastolic volumes (LVESV and LVEDV) were calculated using the biplane method of disks (modified Simpson's rule), or monoplane in the apical 4-chamber window if the apical 2-chamber view was of insufficient quality for reliable analysis. CRT response was defined as an absolute increase of LVEF $\geq 5 \%$, after 6 months of CRT compared to baseline.

\section{Statistical analysis}

All analyses were carried out in GraphPad Prism 8 (GraphPad Software, San Diego, USA).

For the whole cohort, differences between baseline/CRT-OFF/CRT-ON were evaluated using a one-way repeated measures analysis of variance (ANOVA), followed by Bonferroni's multiple comparisons test. Parameters with only baseline and one follow-up value (e.g. LVEF) were assessed using a paired t-test.

Differences between and within the HIGH and LOW subgroups were evaluated using two-way mixed effects ANOVA followed by Bonferroni's multiple comparisons test. For LVEF and LVESV change and patient characteristics that are continuous variables (e.g. age), an unpaired t-test was employed.

Categorical variables were compared using Fisher's exact test, except for left ventricular (LV) lead locations, due to low patient numbers in multiple categories. For New York Heart Association class comparison between the two subgroups, class I \& II and III \& IV were combined to increase the number of patients per category.

Where appropriate, we applied the Geisser-Greenhouse correction during ANOVA to account for possible violations of the assumption of sphericity. $P$ values $\leq 0.05$ were considered statistically significant. Data are shown as mean \pm standard deviation or median (interquartile range). 


\section{Results}

In total, for 80 patients a complete set of baseline, follow-up CRT-ON and follow-up CRTOFF ECGs was obtained. Four patients were excluded because of different QRS morphology during intrinsic conduction at baseline and at follow-up (due to e.g. intermittent left bundle branch block (LBBB)).

In 15 patients only four-chamber apical views of good quality could be obtained during echocardiography. Therefore, monoplane LV volumes and LVEF were determined for these patients. Echocardiograms were not obtained in 5 patients.

Paired Holter recordings were available for 18 patients in the LOW subgroup and 22 patients in the HIGH subgroup. Device readouts regarding ventricular arrhythmias ( $N=31 \mathrm{LOW}, \mathrm{N}=27$ $\mathrm{HIGH}$ ) and premature ventricular contraction (PVC) runs ( $\mathrm{N}=12 \mathrm{LOW}, \mathrm{N}=18 \mathrm{HIGH})$ were also obtained. A ventricular run was defined as 3 or more consecutive PVCs.

Patients were $67 \pm 9$ years old and 33 (43\%) were female. Heart failure aetiology was ischemic in 40 patients (53\%). LBBB was present in 53 patients (70\%) and six patients received an upgrade because of previous right ventricular (RV) pacing. 14 patients (18\%) had atrial fibrillation. More detailed patient characteristics are presented in Table 1.

The LV lead was most commonly placed in an anterolateral $(n=37)$ or inferolateral $(n=25)$ position. The RV lead was placed in the apex in almost all patients, except for one patient in whom the lead was inserted in a septal position.

\section{Entire patient cohort}

LVEF significantly increased from $25 \pm 7 \%$ at baseline to $34 \pm 10 \%$ at follow-up (Figure 1A). Forty-eight patients (68\%) were classified as echocardiographic responders. LVESV decreased significantly, from $140 \pm 47 \mathrm{ml}$ at baseline to $105 \pm 46 \mathrm{ml}$ at follow-up.

QRS duration, QRS area and QRS amplitude all decreased significantly during CRT-ON compared to baseline, while QRS area and amplitude decreased slightly but significantly during CRT-OFF (Figure 1B and Table 2). 
TABLE 1 Patient characteristics

\begin{tabular}{|c|c|c|c|}
\hline & All patients $(n=76)$ & LOW $(n=38)$ & HIGH $(n=38)$ \\
\hline Age (years) & $67 \pm 9$ & $67 \pm 9$ & $66 \pm 9$ \\
\hline Female (n, \%) & $33(43)$ & $18(47)$ & $15(39)$ \\
\hline BMI (kg/m2) & $26 \pm 5$ & $26 \pm 6$ & $27 \pm 5$ \\
\hline \multicolumn{4}{|l|}{ NYHA class } \\
\hline I $(\mathrm{n}, \%)$ & $6(8)$ & $0(0)$ & $6(16)$ \\
\hline II (n, \%) & 45 (59) & $23(61)$ & $22(58)$ \\
\hline III $(\mathrm{n}, \%)$ & $24(32)$ & 15 (39) & $9(24)$ \\
\hline IV $(\mathrm{n}, \%)$ & $1(1)$ & $0(0)$ & $1(3)$ \\
\hline $\begin{array}{l}\text { Ischemic cardiomyopathy } \\
\text { (n, \%) }\end{array}$ & $40(53)$ & $23(61)$ & $17(45)$ \\
\hline $\operatorname{AF}(n, \%)$ & $14(18)$ & $10(26)$ & $4(11)$ \\
\hline LBBB $(n, \%)$ & $53(70)$ & $24(63)$ & $29(76)$ \\
\hline Previous RV pacing (n, \%) & $6(8)$ & $3(8)$ & $3(8)$ \\
\hline ACE-inhibitor/ARB $(n, \%)$ & $69(91)$ & $37(97)$ & $32(84)$ \\
\hline Beta blocker $(n, \%)$ & $71(93)$ & $35(92)$ & $36(95)$ \\
\hline Aldosterone antagonist (n,\%) & $22(29)$ & $12(32)$ & $10(26)$ \\
\hline Loop diuretic $(n, \%)$ & $47(62)$ & $30(79)$ & 17 (45)\# \\
\hline CRT Responders ( $n, \%)$ & $48(68)$ & $23(64)$ & $25(71)$ \\
\hline $\begin{array}{l}\text { Biventricular pacing } \\
(\%, \text { median (IQR)) }\end{array}$ & $99.1(97.5-99.9)$ & $99.1(97.0-99.9)$ & $99.2(98.1-99.7)$ \\
\hline \multicolumn{4}{|l|}{ LV lead location $(n, \%)$} \\
\hline Basal anterior & $1(1)$ & $0(0)$ & $1(3)$ \\
\hline Basal inferolateral & $8(11)$ & $6(16)$ & $2(5)$ \\
\hline Basal anterolateral & $15(20)$ & $7(18)$ & $8(21)$ \\
\hline Mid inferior & $4(5)$ & $3(8)$ & $1(3)$ \\
\hline Mid inferolateral & $17(22)$ & $7(18)$ & $10(26)$ \\
\hline Mid anterolateral & $22(29)$ & $13(34)$ & $9(24)$ \\
\hline Apical lateral & $9(12)$ & $2(5)$ & $7(18)$ \\
\hline
\end{tabular}

Patient characteristics for the entire patient cohort and for the subgroups of patients with $T$ area change after 6 months during CRT-OFF that was smaller (LOW) or larger than the median (HIGH). Variables are shown as mean \pm standard deviation or as number of patients (percentage of patients). AF = atrial fibrillation, $A C E=$ angiotensin converting enzyme, $A R B=$ angiotensin receptor blocker, $B M I=$ body mass index, $C R T=$ cardiac resynchronization therapy, $I Q R=$ interquartile range, $L B B B=$ left bundle branch block, $L V=$ left ventricular, NYHA = New York Heart Association, $R V=$ right ventricular. \# = significant difference between the LOW and HIGH subgroups. 
Compared to baseline, $\mathrm{T}$ area and $\mathrm{T}$ amplitude decreased significantly during CRT-ON and increased significantly during CRT-OFF (Figure 1C, Table 2). The change in $\mathrm{T}$ area between baseline and CRT-OFF did not differ between echocardiographic responders and nonresponders ( $48.0 \pm 47.5$ vs $41.8 \pm 22.9 \mathrm{mV} \cdot \mathrm{ms}, \mathrm{p}>0.05)$. From baseline, QTc interval only increased significantly during CRT-OFF, whereas JTc interval increased significantly during both CRT-ON and CRT-OFF (Table 2). Compared to baseline, $T_{p-e}$ increased during CRT-OFF while precordial QT dispersion did not change compared to baseline in either condition (Table 2).

Vectorcardiographic azimuth and elevation of QRS complex and T wave hardly changed during CRT-OFF compared to baseline (Table 2). During CRT-ON the main QRS azimuth and elevation changed significantly, denoting a move upwards and to the right, while the main $T$ axis moved downwards and to the left, causing a small but significant reduction in spatial peak QRS-T angle (Table 2).

Device readouts showed only a small number of ventricular arrhythmias (none in most patients), both after 2 weeks and 6 months of CRT. Data from devices and Holter recordings demonstrated that the number of PVC runs per hour did not differ between the early time point (baseline or two weeks) and the late time point (6 months) (device: $0.7(0.1-2.8)$ at 2 weeks vs. $0.5(0.0-2.2)$ at 6 months, $p>0.05$; Holter 0.0 (0.0-0.1) at baseline vs. 0.1 (0.0-0.3) at 6 months, $p>0.05)$.

\section{Stratification by $\mathrm{T}$ area change}

The median increase in T area between baseline and the follow-up CRT-OFF measurement was $41 \mathrm{mV} \cdot \mathrm{ms}$. Patient characteristics were comparable between the subgroups with a change in $\mathrm{T}$ area less than and more than $41 \mathrm{mV} \cdot \mathrm{ms}$ (LOW and HIGH subgroups, respectively), except for the larger proportion of patients receiving a loop diuretic in the LOW subgroup (Table 1). Baseline VCG parameters, LVEF and LVESV (LOW $143 \pm 49$ vs. HIGH $136 \pm 46$ ml, p > 0.05) were also comparable (Figure 2A-D, Figure 3A, Table 3).

Patients in the HIGH subgroup had a significantly larger T area during CRT-OFF than patients in the LOW subgroup, despite similar T area during CRT-ON (Figure 2A). In the LOW subgroup T amplitude did not increase significantly during CRT-OFF as compared to baseline, while for the HIGH subgroup, an increase occurred (Table 3).

QRS area and amplitude remained unchanged between baseline and CRT-OFF in the HIGH subgroup, but in the LOW subgroup, a significant decrease was observed (Figure 2B, Table 3). Together with the slightly lower baseline QRS area in the LOW subgroup this created a significant difference in QRS area (Figure 2B) and amplitude (Table 3) between the subgroups during CRT-OFF. This difference was not visible during CRT-ON. 
TABLE 2 ECG and VCG variables for the entire patient cohort

\begin{tabular}{|c|c|c|c|}
\hline & BL & OFF & ON \\
\hline Heart rate $(\mathrm{bpm})$ & $71 \pm 12$ & $65 \pm 11^{\star}$ & $67 \pm 10^{\star}$ \\
\hline QRS duration (ms) & $181 \pm 24$ & $179 \pm 25$ & $154 \pm 24^{*}$ \\
\hline QRS amplitude (mV) & $1.7 \pm 0.5$ & $1.6 \pm 0.5^{\star}$ & $1.1 \pm 0.4^{\star}$ \\
\hline T amplitude (mV) & $0.5 \pm 0.2$ & $0.7 \pm 0.3^{*}$ & $0.3 \pm 0.1^{*}$ \\
\hline QRS/T Area ratio & $1.7 \pm 0.7$ & $1 \pm 0.3^{\star}$ & $2.1 \pm 1.9$ \\
\hline$T_{p-e}(m s)$ & $81 \pm 21$ & $96 \pm 16^{\star}$ & $76 \pm 16$ \\
\hline Precordial QT dispersion (ms) & $19 \pm 12$ & $17 \pm 9$ & $18 \pm 11$ \\
\hline QTc (ms) & $440 \pm 34$ & $467 \pm 32^{\star}$ & $440 \pm 31$ \\
\hline JTc (ms) & $250 \pm 33$ & $284 \pm 25^{\star}$ & $281 \pm 29^{*}$ \\
\hline Azimuth QRS (degrees) & $294 \pm 24$ & $282 \pm 39^{*}$ & $215 \pm 61^{*}$ \\
\hline Elevation QRS (degrees) & $85 \pm 24$ & $88 \pm 24^{*}$ & $120 \pm 31^{*}$ \\
\hline Azimuth T (degrees) & $100 \pm 45$ & $102 \pm 61$ & $18 \pm 73^{\star}$ \\
\hline Elevation T (degrees) & $92 \pm 23$ & $94 \pm 24$ & $57 \pm 30^{\star}$ \\
\hline Spatial peak QRS-T angle (degrees) & $160 \pm 17$ & $159 \pm 13$ & $140 \pm 33^{*}$ \\
\hline
\end{tabular}

Values are shown as mean \pm standard deviation. ${ }^{*}=p \leq 0.05$ vs. BL. $T_{p-e}=T$ peak-to-end interval. For further abbreviations see legend Figure 2.
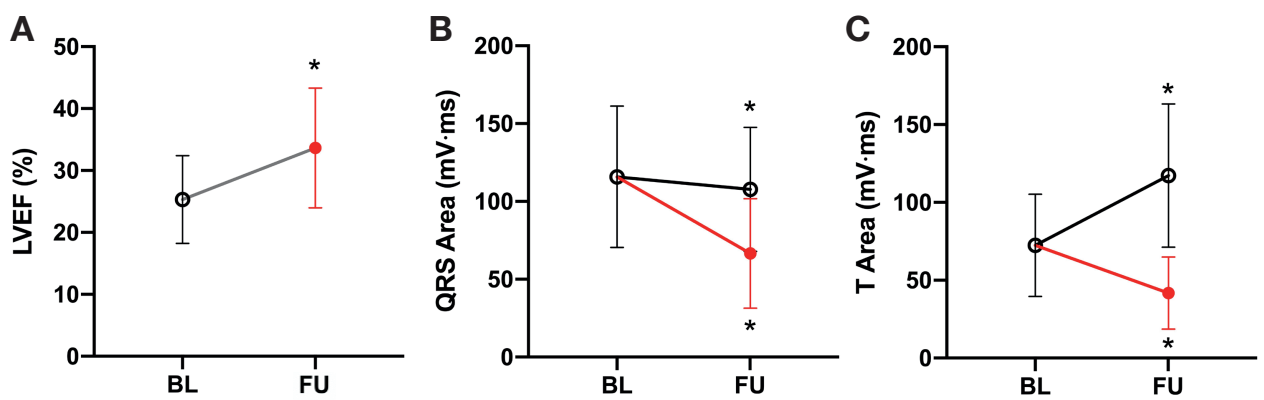

FIGURE 1. A. LVEF at baseline and during CRT-ON at follow-up. B. QRS area during CRT-OFF (black symbols) and CRT-ON (red symbol). C. T area during CRT-OFF and CRT-ON. * $=p \leq 0.05$ vs. BL. BL $=$ baseline, CRT $=$ cardiac resynchronization therapy, FU = six-month follow-up, LVEF = left ventricular ejection fraction. 
A

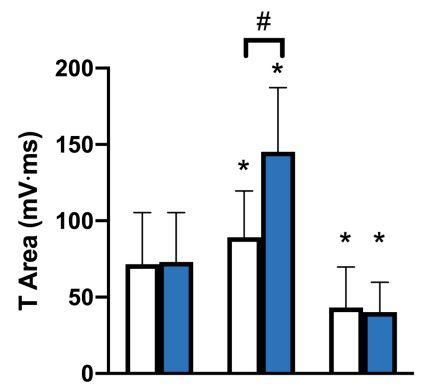

C

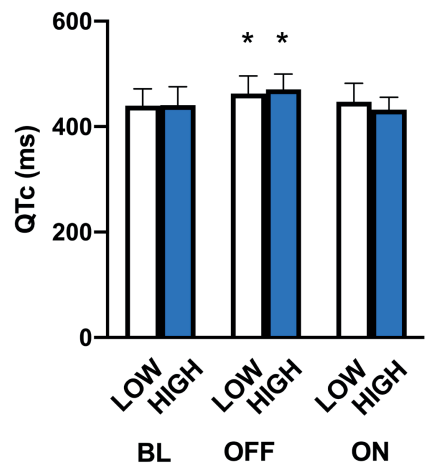

B

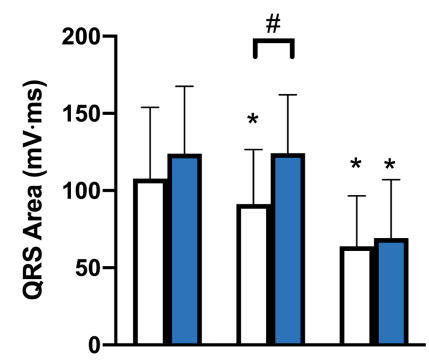

D

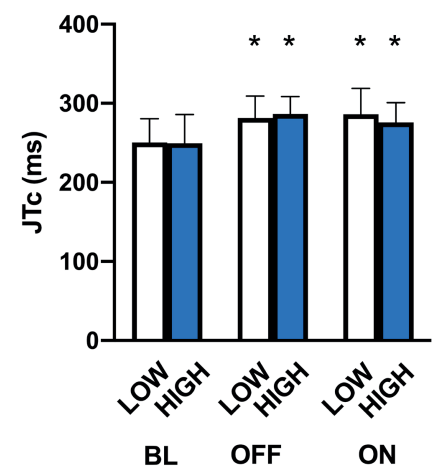

FIGURE 2. VCG and ECG parameters during CRT-OFF and CRT-ON in the patient subgroups with $T$ area change smaller than median (LOW, white bars) and T area change larger than median (HIGH, blue bars). A. T area. B. QRS area. C. QTc interval. D. JTc interval. \# = p $\leq 0.05$ for LOW vs. HIGH in the indicated measurement; ${ }^{*}=p \leq 0.05$ vs. BL. $\mathrm{BL}$ = baseline, $\mathrm{CRT}$ = cardiac resynchronization therapy, ECG = electrocardiographic, VCG = vectorcardiographic, OFF and ON denote CRT-OFF and CRT-ON, respectively, at six-month follow-up.

Between the subgroups, no significant differences in QRS duration, QTc, JTc, $T_{p-e}$ and precordial QT dispersion were observed at any time point (Figure 2C and D, Table 3). QRS and T vector angles were also comparable for both subgroups at all time points, although QRS-T angle decreased significantly between baseline and CRT-ON only in the HIGH subgroup (Table 3).

LVEF values were not significantly different between the two subgroups at baseline, but at follow-up, patients in the HIGH subgroup had a significantly higher LVEF than patients in the LOW subgroup (Figure 3A). This difference was also reflected in the significantly larger increase in LVEF in the HIGH subgroup (Figure 3B). For LVESV, no significant differences were observed between the LOW and the HIGH subgroups at follow-up (111 \pm 53 vs. $98 \pm 38 \mathrm{ml}$ respectively, $p>0.05)$. Change in LVESV between baseline and follow-up measurements did not differ significantly between the two subgroups either (LOW $-32 \pm 30 \mathrm{ml}$ vs. HIGH $-38 \pm 30 \mathrm{ml}, \mathrm{p}>$ 0.05). 
TABLE 3 ECG and VCG variables stratified according to T area change

\begin{tabular}{|c|c|c|c|c|c|c|}
\hline & BL LOW & BL HIGH & OFF LOW & OFF HIGH & ON LOW & ON HIGH \\
\hline Heart rate (bpm) & $74 \pm 11$ & $68 \pm 13$ & $67 \pm 13^{*}$ & $63 \pm 9$ & $69 \pm 9^{*}$ & $64 \pm 9 \#$ \\
\hline QRS duration (ms) & $178 \pm 25$ & $184 \pm 22$ & $176 \pm 27$ & $182 \pm 24$ & $154 \pm 29^{*}$ & $154 \pm 19$ \\
\hline QRS amplitude $(\mathrm{mV})$ & $1.7 \pm 0.5$ & $1.7 \pm 0.5$ & $1.4 \pm 0.4^{*}$ & $1.8 \pm 0.4 \#$ & $1 \pm 0.4^{*}$ & $1.1 \pm 0.5^{\star}$ \\
\hline T amplitude $(\mathrm{mV})$ & $0.5 \pm 0.3$ & $0.5 \pm 0.2$ & $0.6 \pm 0.2$ & $0.9 \pm 0.2^{*} \#$ & $0.3 \pm 0.2^{*}$ & $0.3 \pm 0.1^{\star}$ \\
\hline QRS/T Area ratio & $1.6 \pm 0.5$ & $1.9 \pm 0.8$ & $1 \pm 0.3^{\star}$ & $0.9 \pm 0.2^{*}$ & $1.9 \pm 1.9$ & $2.2 \pm 1.8$ \\
\hline$T_{p-e}(m s)$ & $80 \pm 14$ & $81 \pm 26$ & $93 \pm 16^{*}$ & $99 \pm 16^{*}$ & $79 \pm 15$ & $73 \pm 17$ \\
\hline Precordial QT dispersion (ms) & $18 \pm 9$ & $20 \pm 15$ & $16 \pm 9$ & $17 \pm 10$ & $17 \pm 10$ & $19 \pm 11$ \\
\hline Azimuth QRS (degrees) & $288 \pm 27$ & $301 \pm 20$ & $275 \pm 51$ & $289 \pm 19^{*}$ & $210 \pm 73^{*}$ & $220 \pm 46^{*}$ \\
\hline Elevation QRS (degrees) & $89 \pm 26$ & $81 \pm 21$ & $93 \pm 28$ & $83 \pm 18$ & $120 \pm 36^{*}$ & $120 \pm 25^{\star}$ \\
\hline Azimuth T (degrees) & $91 \pm 49$ & $108 \pm 40$ & $92 \pm 69$ & $111 \pm 51$ & $13 \pm 73^{*}$ & $24 \pm 73^{*}$ \\
\hline Elevation $\mathrm{T}$ (degrees) & $88 \pm 24$ & $97 \pm 21$ & $90 \pm 25$ & $98 \pm 22$ & $54 \pm 31^{*}$ & $61 \pm 29^{*}$ \\
\hline $\begin{array}{l}\text { Spatial peak QRS-T } \\
\text { angle (degrees) }\end{array}$ & $156 \pm 21$ & $163 \pm 11$ & $159 \pm 14$ & $160 \pm 13$ & $146 \pm 34$ & $134 \pm 31$ \\
\hline
\end{tabular}

Values are shown as mean \pm standard deviation. LOW denotes patients with $T$ area change smaller than median, HIGH denotes patients with $T$ area change larger than median. \# = $p \leq 0.05$ for LOW vs. HIGH in the indicated

A

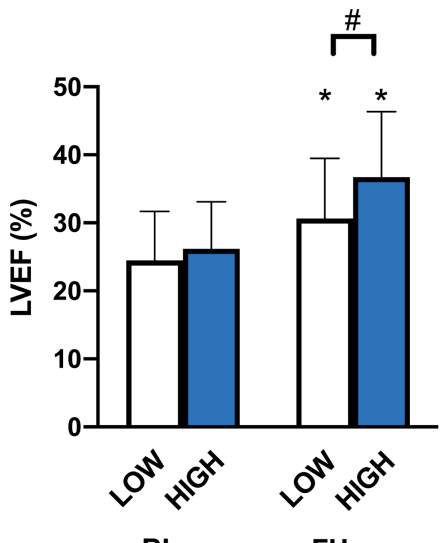

BL
B

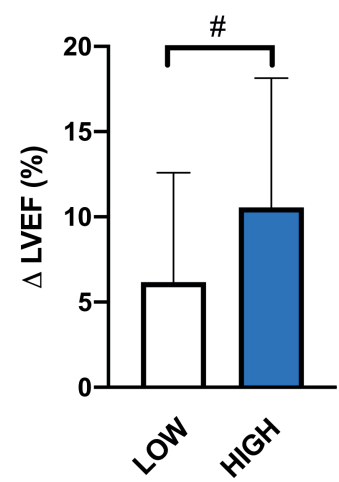

FIGURE 3. A. LVEF in the patient subgroups with T area change smaller than median (LOW, white bars) and T area change larger than median (HIGH, blue bars). B. LVEF change between baseline and follow-up in the two subgroups. $\#=p \leq 0.05$ for LOW vs. HIGH in the indicated measurement; ${ }^{*}=p \leq 0.05$ vs. BL. BL = baseline, FU = six-month follow-up, LVEF = left ventricular ejection fraction. 
Neither the number of PVC runs per hour (Holter baseline LOW 0.0 (0.0 - 0.1), baseline HIGH 0.0 (0.0 - 0.1), 6 months LOW 0.1 (0.0 - 0.2), 6 months HIGH 0.1 (0.0 - 0.3); device 2 weeks LOW 0.7 (0.2 - 1.5), 2 weeks HIGH 0.7 (0.1 - 7.2), 6 months LOW 0.7 (0.0 - 2.0), 6 months $\mathrm{HIGH} 0.4(0.0-2.2))$ nor the number of ventricular arrhythmia episodes differed between the two subgroups at either baseline, 2 weeks or 6 months.

When we stratified the patients by echocardiographic response to CRT, we did not observe a difference in PVC runs from Holter or device between these two subgroups.

\section{Discussion}

The main findings of our study are that repolarization remodelling after CRT (characterized by a larger increase in T area) is associated with echocardiographically measured response and that in this cohort (with few arrhythmias) no association with the risk of ventricular arrhythmia following CRT was observed.

\section{Ejection fraction and repolarization change}

While T area changes after CRT have been reported before ${ }^{6}$, to the best of our knowledge our study is the first to assess the link between $T$ area change and echocardiographically measured CRT response.

The exact mechanisms behind the link between T wave changes and CRT response cannot be derived from this study, although in general it can be stated that hearts that show adaptive capacity with regard to contraction also do so with regard to electrophysiology ${ }^{4,5}$. While contractile and electrical remodelling may not be based on a common mechanism, it is interesting to note that both computer simulation studies ${ }^{11}$ and animal experiments ${ }^{12}$ have indicated a possible link between calcium currents and cardiac remodelling during CRT. Calcium currents play an important role in both repolarization and contraction. The increase in $\mathrm{T}$ area that we observe during CRT-OFF (chapter 3) seems to be in line with computer simulations that assume a kind of mechano-electrical feedback: weakening of contraction, as is the case in early activated regions, is counteracted by an increase in calcium influx ${ }^{11}$. This increased influx also causes action potential duration (APD) prolongation, while the opposite phenomena occur in late activated regions ${ }^{11}$. It is therefore tempting to speculate that these phenomena may at least contribute to the link we found between $T$ area change and ejection fraction increase. Studies in patients, however, have both shown prolongation ${ }^{13}$ and shortening ${ }^{14}$ of the activation recovery interval (ARI, a surrogate for APD) in zones of early activation during RV pacing or CRT. In the latter study, CRT responders showed a reduction of ARI close to the pacing site, while non-responders showed an increase ${ }^{14}$. The time scale at which these 
changes occurred (weeks to months) ${ }^{14}$, however, is considerably longer than the time scale at which $T$ wave changes after CRT have been reported (days to weeks, chapter 3), meaning that this may represent a different mechanism.

Furthermore, other mechanisms (e.g. asymmetric hypertrophy, which develops during LBBB and disappears during $\mathrm{CRT}^{15,16}$ ) may also affect $\mathrm{T}$ wave amplitude and may thus play a role ${ }^{17}$. Summarizing, the exact mechanisms are still unclear, but the link between electrical and mechanical remodelling has been demonstrated from a variety of angles.

\section{Differences in de- and repolarization parameters}

Of all repolarization parameters we studied, only $\mathrm{T}$ area and amplitude were significantly different between the LOW and the HIGH patient subgroups, and only during CRT-OFF. These observations are in line with an earlier study by Lellouche et al. that showed that QTc measured after one year of CRT did not differ between responders and non-responders ${ }^{18}$. This study, however, found that $T_{p-e}$ was shorter in responders ${ }^{18}$. This finding is corroborated by another study, demonstrating (during CRT-ON) a shorter $T_{p-e}$ in CRT responders compared to nonresponders after 3 months of $C R T^{19}$. In our study, $\mathrm{T}_{\mathrm{p} \text {-e }}$ was not significantly changed during CRT-ON, not even in the HIGH subgroup, that showed a better echocardiographic response than the LOW subgroup.

On the other hand, $T_{p-e}$ significantly increased during CRT-OFF, just like $T$ area. This indicates that turning CRT off may be a relevant provocation test to unravel changes in repolarization after CRT. This also holds true for the increases in QTC and JTC.

Interestingly, during CRT-OFF measurements not only T area, but also QRS area and amplitude were different between the LOW and HIGH subgroups. In particular, QRS area and amplitude decreased significantly in the LOW subgroup, which showed a smaller increase in LVEF than the HIGH subgroup. This observation suggests changes in ventricular activation over the six-month follow-up period, unveiled by the CRT-OFF procedure. Because QRS duration did not change significantly during CRT-OFF, total activation is not likely to have changed. The reduction in QRS amplitude in the LOW subgroup indicates a reduction in unopposed electrical forces, possibly caused by a more irregular pattern of conduction. Potential causes of the latter may be negative tissue remodelling (e.g. due to cellular uncoupling or the loss of viable myocardium $)^{20,21}$, which may relate to the poorer response to CRT in this subgroup.

\section{Arrhythmia risk}

A link between echocardiographic CRT response and ventricular arrhythmia incidence has been found in several studies, with responders generally showing a lower arrhythmia risk ${ }^{4,5,19,22-}$ ${ }^{24}$. This indirectly suggests that tissue remodelling and arrhythmia risk are inversely related. 
In our study, however, no difference was found in arrhythmia incidence, neither between the LOW and HIGH subgroups nor between responders and non-responders. The most important explanation for this observation is the relative rarity of arrhythmias in our cohort, thus lowering the statistical power to find any significant differences.

Out of 58 patients for whom device data was available, 1 patient experienced ventricular fibrillation and 5 experienced ventricular tachycardia. This incidence is relatively comparable to the findings of the REVERSE trial (14\% of patients experienced any type of arrhythmia) ${ }^{24}$, but somewhat lower than in the study by Itoh (20\% of patients experienced some kind of arrhythmia) ${ }^{19}$. Possibly, the differences in NYHA class between the studies played a role: in our study, the majority of patients was in NYHA class I and II, while in the study by Itoh, patients were in NYHA class III or IV.

Based on the link between repolarization dispersion and arrhythmias, we also investigated whether there was a connection between the latter and T area change. However, even when comparing the 5 patients with the largest number of ventricular runs per hour on Holter after 6 months with the other patients for whom Holters were available, no differences in $T$ area were seen at any of the time points (data not shown).

In conclusion, although the majority of patients in our cohort show a significant increase in T area when temporarily turning off CRT (indicating changes in ventricular repolarization, see also chapter 3 ) these repolarization changes may not directly relate to the (rare) occurrence of arrhythmias.

\section{Limitations}

This study has several limitations. Firstly, the sample size was relatively small. This particularly impacts the evaluation of the data of the infrequent ventricular arrhythmias. The observational nature of the study implies that e.g. device settings and medications were adjusted as judged necessary during the study period. Further studies are required to determine the optimum parameter to identify post-CRT electrical remodelling.

\section{Conclusions}

A larger degree of repolarization remodelling may be linked to a stronger echocardiographically measured response to CRT. This effect occurred during a virtual absence of arrhythmias. 


\section{Acknowledgements}

This study was supported by the Swedish Heart-Lung Foundation and the Swedish Society of Medicine. 


\section{References}

1. Brignole M, Auricchio A, Baron-Esquivias G, Bordachar P, Boriani G, Breithardt O-A, et al. 2013 ESC Guidelines on cardiac pacing and cardiac resynchronization therapy. Eur Heart J 2013;34:2281329.

2. Medina-Ravell VA, Lankipalli RS, Yan G-X, Antzelevitch C, Medina-Malpica NA, Medina-Malpica $\mathrm{OA}$, et al. Effect of epicardial or biventricular pacing to prolong QT interval and increase transmural dispersion of repolarization: does resynchronization therapy pose a risk for patients predisposed to long QT or torsade de pointes? Circulation 2003;107:740-6.

3. Fish JM, Diego JM Di, Nesterenko V, Antzelevitch C. Epicardial Activation of Left Ventricular Wall Prolongs QT Interval and Transmural Dispersion of Repolarization: Implications for Biventricular Pacing. Circulation 2004;109:2136-42.

4. Saini A, Kannabhiran M, Reddy P, Gopinathannair R, Olshansky B, Dominic P. Cardiac Resynchronization Therapy May Be Antiarrhythmic Particularly in Responders: A Systematic Review and Meta-Analysis. JACC Clin Electrophysiol 2016;2:307-16.

5. Deif B, Ballantyne B, Almehmadi F, Mikhail M, Mclntyre WF, Manlucu J, et al. Cardiac resynchronization is pro-arrhythmic in the absence of reverse ventricular remodelling: A systematic review and metaanalysis. Cardiovasc Res 2018;114:1435-44.

6. Wecke L, Deursen CJM Van, Bergfeldt L, Prinzen FW. Repolarization changes in patients with heart failure receiving cardiac resynchronization therapy - Signs of cardiac memory. J Electrocardiol 2011;44:590-8.

7. Huysduynen BH Van, Swenne CA, Draisma HHM, Antoni ML, Vooren H Van De, Wall EE Van Der, et al. Validation of ECG indices of ventricular repolarization heterogeneity: A computer simulation study. J Cardiovasc Electrophysiol 2005;16:1097-103.

8. Engels EB, Alshehri S, Deursen CJM Van, Wecke L, Bergfeldt L, Vernooy K, et al. The synthesized vectorcardiogram resembles the measured vectorcardiogram in patients with dyssynchronous heart failure. J Electrocardiol 2015;48:586-92.

9. Hermans BJM, Vink AS, Bennis FC, Filippini LH, Meijborg VMF, Wilde AAM, et al. The development and validation of an easy to use automatic QT-interval algorithm. PLoS One 2017;12:e0184352.

10. Vandenberk B, Vandael E, Robyns T, Vandenberghe J, Garweg C, Foulon V, et al. Which QT Correction Formulae to Use for QT Monitoring? J Am Heart Assoc 2016;5:e003264.

11. Kuijpers NHL, Hermeling E, Lumens J, Eikelder HMM ten, Delhaas T, Prinzen FW. Mechano-electrical coupling as framework for understanding functional remodeling during LBBB and CRT. Am J Physiol - Hear Circ Physiol 2014;306:1644-59.

12. Aiba T, Hesketh GG, Barth AS, Liu T, Daya S, Chakir K, et al. Electrophysiological consequences of dyssynchronous heart failure and its restoration by resynchronization therapy. Circulation 2009;119:1220-30.

13. Marrus SB, Andrews CM, Cooper DH, Faddis MN, Rudy Y. Repolarization Changes Underlying LongTerm Cardiac Memory Due to Right Ventricular Pacing. Circ Arrhythmia Electrophysiol 2012;5:77381. 
14. Chen Z, Hanson B, Sohal M, Sammut E, Child N, Shetty A, et al. Left ventricular epicardial electrograms show divergent changes in action potential duration in responders and nonresponders to cardiac resynchronization therapy. Circ Arrhythmia Electrophysiol 2013;6:265-71.

15. Middendorp LB van, Kuiper M, Munts C, Wouters P, Maessen JG, Nieuwenhoven FA van, et al. Local microRNA-133a downregulation is associated with hypertrophy in the dyssynchronous heart. ESC Hear Fail 2017;4:241-51.

16. Vernooy K, Cornelussen RNM, Verbeek XAAM, Vanagt WYR, Hunnik A Van, Kuiper M, et al. Cardiac resynchronization therapy cures dyssynchronopathy in canine left bundle-branch block hearts. Eur Heart J 2007;28:2148-55.

17. Dilaveris P, Gialafos E, Poloniecki J, Hnatkova K, Richter D, Andrikopoulos G, et al. Changes of the T-wave amplitude and angle: an early marker of altered ventricular repolarization in hypertension. Clin Cardiol 2000;23:600-6.

18. Lellouche N, Diego C De, Boyle NG, Wiener I, Akopyan G, Child JS, et al. Relationship between mechanical and electrical remodelling in patients with cardiac resynchronization implanted defibrillators. Europace 2011;13:1180-7.

19. Itoh M, Yoshida A, Fukuzawa K, Kiuchi K, Imamura K, Fujiwara R, et al. Time-dependent effect of cardiac resynchronization therapy on ventricular repolarization and ventricular arrhythmias. Europace 2013;15:1798-804.

20. Nguyên UC, Claridge S, Vernooy K, Engels EB, Razavi R, Rinaldi CA, et al. Relationship between vectorcardiographic QRS area, myocardial scar quantification, and response to cardiac resynchronization therapy. J Electrocardiol 2018;51:457-63.

21. Potse M, Krause D, Bacharova L, Krause R, Prinzen FW, Auricchio A. Similarities and differences between electrocardiogram signs of left bundle-branch block and left-ventricular uncoupling. Europace 2012;14:v33-9.

22. Thijssen J, Borleffs CJW, Delgado V, Rees JB Van, Mooyaart EAQ, Bommel RJ Van, et al. Implantable cardioverter-defibrillator patients who are upgraded and respond to cardiac resynchronization therapy have less ventricular arrhythmias compared with nonresponders. J Am Coll Cardiol 2011;58:2282-9.

23. Barsheshet A, Wang PJ, Moss AJ, Solomon SD, Al-Ahmad A, McNitt S, et al. Reverse remodeling and the risk of ventricular tachyarrhythmias in the MADIT-CRT (Multicenter Automatic Defibrillator Implantation Trial-Cardiac Resynchronization Therapy). J Am Coll Cardiol 2011;57:2416-23.

24. Gold MR, Linde C, Abraham WT, Gardiwal A, Daubert JC. The impact of cardiac resynchronization therapy on the incidence of ventricular arrhythmias in mild heart failure. Hear Rhythm 2011;8:67984. 


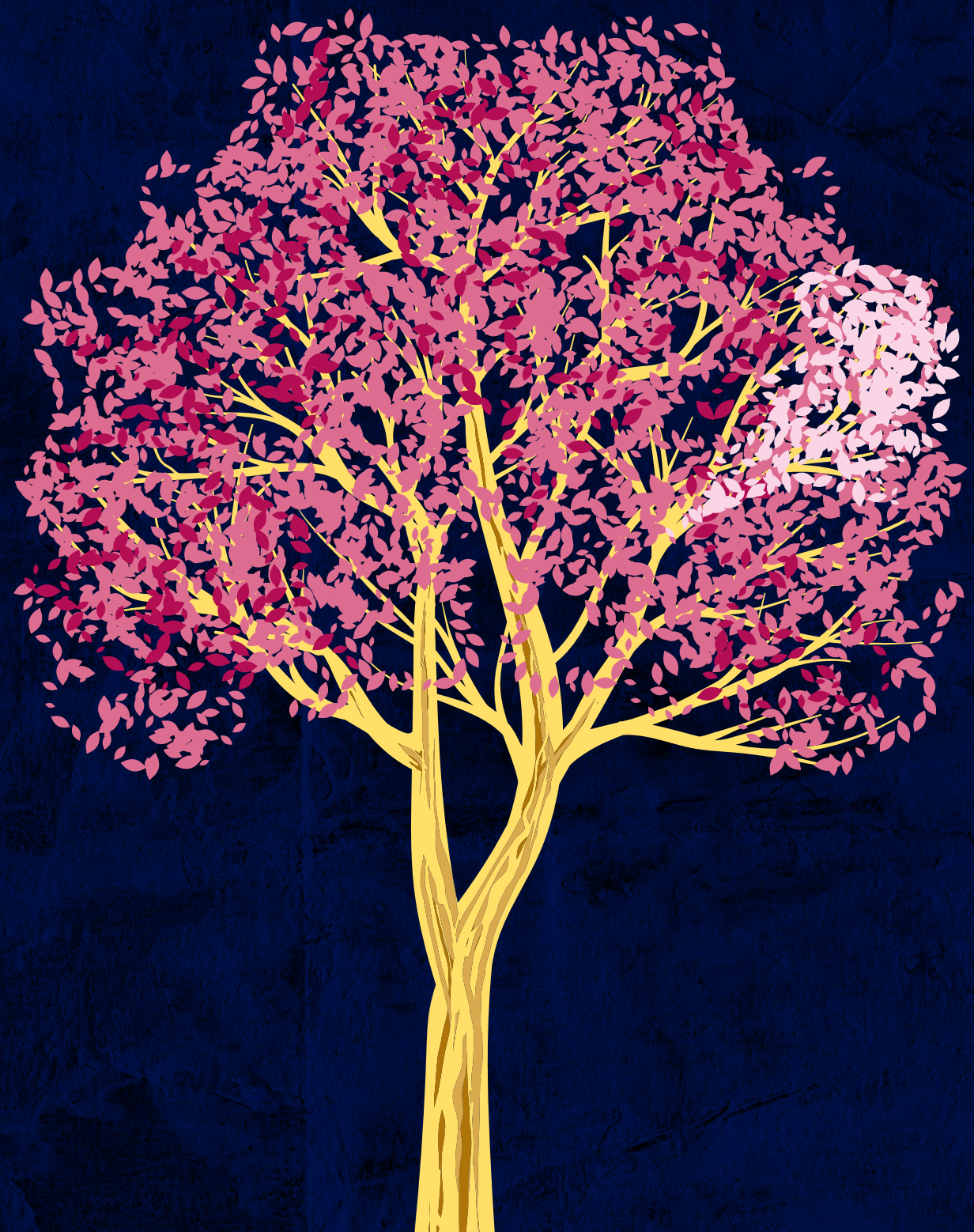




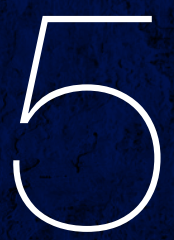

\section{Can CRT beneficially impact cardiac function in the presence of structural mitral regurgitation?}

Nienke J. Verzaal ${ }^{1}$, Lars B. van Middendorp ${ }^{2}$, Marion Kuiper ${ }^{1}$, Arne van Hunnik ${ }^{1}$, Caroline J.M. van Deursen ${ }^{3}$, Tammo Delhaas ${ }^{4}$ and Frits W. Prinzen ${ }^{1}$

\footnotetext{
'Department of Physiology, Maastricht University, Maastricht, The Netherlands

${ }^{2}$ Department of Cardiothoracic Surgery, Maastricht University Medical Centre, Maastricht, The Netherlands ${ }^{3}$ Department of Cardiology, Maastricht University Medical Centre, Maastricht, The Netherlands

${ }^{4}$ Department of Biomedical Engineering, Maastricht University, Maastricht, The Netherlands
} 


\section{Abstract}

Background Cardiac resynchronization therapy (CRT) has well-described beneficial effects on dyssynchronous heart failure and may also cause a reduction in functional mitral regurgitation (MR). However, it is not yet known whether CRT may still be beneficial if structural MR is present. Moreover, it is unclear which pacing mode would be most advantageous in this scenario.

Methods In 8 dogs, structural MR was induced through destruction of the chordae tendineae and 4 weeks later left bundle branch block (LBBB) was induced through radiofrequency ablation (MR+LBBB). Eleven dogs with only LBBB served as the control group. Over time, echocardiographic and haemodynamic measurements were performed. After 16 weeks of LBBB haemodynamic measurements were performed during three pacing modes: biventricular $\left(B_{\text {best }}\right)$ pacing, left ventricular (LV) pacing with a short atrioventricular (AV) delay $\left(L V_{\text {short }}\right)$ and LV pacing with an $A V$ delay aimed at fusion with intrinsic activation $\left(\mathrm{LV}_{\text {fusion }}\right)$.

Results LV end-diastolic volume was significantly larger in MR+LBBB dogs than in the LBBB group, while LV end-diastolic pressure also tended to be higher in the former. All pacing modes significantly increased the maximum rate of $\mathrm{LV}$ pressure rise $\left(\mathrm{LVdP} / \mathrm{dt}_{\max }\right)$ in the $\mathrm{LBBB}$ dogs. In the MR+LBBB group BiV ${ }_{\text {best }}$ tended to increase in $L V d P / d t_{\max }(p=0.14)$ and significantly reduced LV end-diastolic pressure $\left(\right.$ LVP $\left._{\text {ed }}\right)$.

Conclusions Despite the presence of structural MR in the MR+LBBB animals, CRT can improve ventricular pump function in LBBB hearts, as evidenced by a moderate increase in $\mathrm{LVdP} / \mathrm{dt}_{\text {max }}$, and a significant decrease in $\mathrm{LVP}_{\text {ed }}$. In this animal model of MR+LBBB the various pacing modes (BiV, $\mathrm{LV}_{\text {short }}$ or $\mathrm{LV}_{\text {fusion }}$ ) result in comparable haemodynamic effects. 


\section{Introduction}

Two types of functional mitral regurgitation (MR) have been described in patients with heart failure: proportionate and disproportionate, reflecting whether the relation between left ventricular end-diastolic volume (LVEDV) and the severity of MR is linear or not ${ }^{1}$. Proportionate MR is caused by dilatation of the left ventricle (LV) with dislocation of the papillary muscles and chordae tendineae, plus dilatation of the mitral annulus ${ }^{1}$. This combination impairs coaptation of the mitral valve leaflets ${ }^{1}$. In disproportionate MR the amount of regurgitation is larger than what would be expected based on LVEDV, suggesting other contributing factors like mitral valve leaflet tethering due to dyssynchronous contraction of the papillary muscles in patients with left bundle branch block (LBBB) ${ }^{1}$.

Cardiac resynchronization therapy (CRT) may beneficially impact MR in ventricular dyssynchrony through several mechanisms ${ }^{2}$. Resynchronization of contraction leads to faster build-up of pressure ${ }^{3}$, while resynchronization of the papillary muscles may result in improved positioning of the valve leaflets ${ }^{4}$, both of which improve valve closure. Moreover, CRT may reduce mitral orifice size $^{5}$ and atrioventricular (AV) dyssynchrony ${ }^{2,6}$, thus further ameliorating MR. These effects can be observed acutely ${ }^{3}$, but also long-term ${ }^{7}$, with reverse remodelling caused by CRT leading to improved coaptation of the mitral valve leaflets through smaller LV volumes and better alignment of papillary muscles and chordae tendineae ${ }^{2}$.

Although these mechanisms all point to a beneficial effect of CRT on MR severity, less is known about whether MR reduction is a crucial element in determining CRT benefit. On the one hand, several studies found that persistence or worsening of MR after CRT may negatively impact cardiac function ${ }^{8,9}$ and (event-free) survival ${ }^{4,9,10}$, with more severe MR having a larger negative impact $^{11,12}$. On the other hand, the exact nature of the link between MR improvement and CRT response is less clear, since a beneficial response to CRT also occurs in the absence of a reduction of $\mathrm{MR}^{4,13,14}$. In these cases, CRT benefit may be derived from, for example, an increase in cardiac function due to ventricular resynchronization or better atrioventricular coupling. Chronically, LV volume and hospitalizations may be reduced, while survival improves ${ }^{15}$. Therefore, we hypothesized that CRT may be of benefit even in the presence of structural MR, which by itself is not thought to be amenable to CRT because valve incompetence arises as a result of structural abnormality of the mitral valve apparatus.

A second question was which pacing mode would be most beneficial in case of structural MR. Three modes would deserve investigation: 1) optimum biventricular (BiV) pacing, 2) LV pacing with AV delay programmed at an intermediate value, thus allowing optimal fusion with intrinsic activation and 3) LV pacing at a short AV delay, thus creating LV pre-excitation. 
It was the aim of the present study to investigate the acute haemodynamic effects of these pacing modes in a novel animal model of chronic MR+LBBB, and to compare the outcomes with those obtained in animals with isolated chronic LBBB. In the MR+LBBB model, MR was created by destruction of chordae tendineae, thus forming a kind of structural MR.

\begin{tabular}{|c|c|}
\hline \multicolumn{2}{|c|}{ Abbreviations and acronyms } \\
\hline $\mathrm{AO}$ & Aortic \\
\hline AV & Atrioventricular \\
\hline BiV $_{\text {best }}$ & Biventricular pacing mode that yielded largest increase in $\mathrm{LVdp} / \mathrm{dt}_{\max }$ \\
\hline $\mathrm{BL}$ & Baseline \\
\hline $\mathrm{dP} / \mathrm{dt}_{\max }$ & Maximum rate of pressure rise \\
\hline EDV & End-diastolic volume \\
\hline EF & Ejection fraction \\
\hline ESV & End-systolic volume \\
\hline IVMD & Interventricular mechanical delay \\
\hline MR & Mitral regurgitation \\
\hline LBBB & Left bundle branch block \\
\hline LV & Left ventricle/ventricular \\
\hline $\mathrm{LV}_{\text {short }}$ & LV pacing with short AV delay \\
\hline $\mathrm{LV}_{\text {fusion }}$ & LV pacing with AV delay aimed at fusion with intrinsic activation \\
\hline$P_{\text {ed }}$ & End-diastolic pressure \\
\hline$P_{\text {pulse }}$ & Pulse pressure \\
\hline$P_{\text {sys }}$ & Systolic pressure \\
\hline RV & Right ventricle/ventricular \\
\hline SV & Stroke volume \\
\hline
\end{tabular}

\section{Materials and Methods}

Animal handling was performed according to the Dutch Law on Animal Experimentation and the European Directive for the Protection of Vertebrate Animals used for Experimental and Other Scientific Purposes (86/609/EU). The protocol was approved by the Experimental Animal Committee of Maastricht University. 


\section{Animal models}

Experiments were performed, as described previously ${ }^{35}$, on adult mongrel dogs of either sex and unknown age, weighing $21.4 \pm 1.7 \mathrm{~kg}$. All experiments were performed under general anaesthesia. Thiopental (300 mg) was administered intravenously to induce anaesthesia, followed by continuous infusion of midazolam $(0.25 \mathrm{mg} / \mathrm{kg} / \mathrm{h})$ and sufentanil $(3 \mu \mathrm{g} / \mathrm{kg} / \mathrm{h})$ for maintenance.

In 8 dogs, MR was induced with closed chest using a customized electrophysiology catheter with a hook at the distal tip. The catheter was inserted into the carotid artery and introduced into the left ventricle, where the hook was used to grasp one or more chorda(e). The catheter was then retracted into a sheath and the chorda(e) was/were destroyed by cauterization. This process was repeated until intraprocedural echocardiographic and fluoroscopic evaluation indicated moderate-severe mitral regurgitation. Four weeks after MR induction LBBB was created by radiofrequency ablation of the left bundle branch during a closed-chest procedure ${ }^{16}$. LBBB was also created in 11 other animals that served as a control group.

\section{Echocardiography}

At baseline and at the end of the protocol, echocardiography was performed using a Vivid 7 system (GE Vingmed, Horten, Norway). Left ventricular internal diameter during diastole (LVIDd) and systole (LVIDs) were determined in the parasternal short-axis section. Using the Teichholz formula, diameters were converted to LVEDV and LV end-systolic volume (LVESV), respectively. Subsequently, LV ejection fraction (LVEF) was calculated. For 1 dog, no sacrifice echocardiogram was available, and therefore this animal was excluded from echocardiographic analysis.

\section{Haemodynamic measurements}

During each surgical procedure and at the final day of the experimental protocol (week 20 in the MR+LBBB group and week 16 in the LBBB group), haemodynamic measurements were performed, using 7-Fr catheter tip manometers (CD-Leycom, Zoetermeer, the Netherlands) that were placed in the LV and right ventricle (RV). 2 dogs from the LBBB group were excluded from this analysis of haemodynamics over time due to missing baseline values.

For the sacrifice experiment, an additional catheter was inserted into the aorta for aortic pressure measurements, which were available for 6 of the MR+LBBB animals and 8 of the LBBB animals. Interventricular mechanical delay (IVMD) was calculated as the time shift that was necessary to maximize the cross-correlation coefficient between the upslopes of the LV and the RV pressure curves ${ }^{16}$. A positive value indicates that the LV upslope occurs first ${ }^{16}$. 
In a subset of animals the following measurements of cardiac output were compared to determine regurgitant fraction: 1) thermodilution using a Swann-Ganz catheter, introduced through the jugular vein and advanced into the pulmonary artery for determination of forward stroke volume (SV) and 2) SV calculated from echocardiography. The regurgitant volume through the mitral valve was then calculated as fraction of total SV:

$$
\text { Regurgitant fraction }=1-\left(\frac{\text { forward } S V}{\text { total } S V}\right)
$$

assuming that SV trough the pulmonary valve is in equilibrium with the SV trough the aortic valve.

\section{Pacing protocol}

Pacing was performed using a transvenously introduced right atrial lead and an RV apical electrode on a transvenously introduced octopolar electrode catheter (Daig Livewire TC, Minnetonka, MN). After opening the thorax, a multielectrode array was wrapped around the ventricles to allow pacing the LV from various sites. In this study only data for pacing a midlateral LV electrode are reported.

Before starting the pacing protocol, animals were haemodynamically stabilized using infusion with saline and dobutamine if necessary. This was particularly required in the MR+LBBB animals.

Atrial pacing (AAl mode) at \pm 10 bpm above the intrinsic heart rate was used as baseline. After determining the intrinsic AV delay from the surface electrocardiogram (ECG), simultaneous BiV and LV only pacing was performed. BiV pacing was performed at AV delays 30 and 10 ms shorter than intrinsic PR interval, to ensure full capture. For analysis we used the setting providing the largest increase in maximum rate of $L V$ pressure rise $\left(\mathrm{LVdP} / \mathrm{dt}_{\max }\right)$ of the two $\left(B V_{\text {best }}\right)$. LV only pacing was performed at a wide range of $A V$ delays, ranging from intrinsic $A V$ delay $-50 \mathrm{~ms}\left(\mathrm{LV}_{\text {short }}\right)$ up to a maximum of $+90 \mathrm{~ms}$ (aiming at fusion pacing) ${ }^{17}$. The AV delay that yielded an IVMD that was closest to the value halfway between that during LBBB and LV pacing at shortest $A V$ delay was selected as $L_{\text {fusion, }}$, because this has been previously shown to provide the best resynchronization ${ }^{17}$. Data were analysed using custom-written MATLAB software (MathWorks, Natick, MA).

\section{Statistical analysis}

All analyses were carried out using GraphPad Prism 8 (GraphPad Software, San Diego, USA). Values of the haemodynamic variables and \% change from baseline (except for LV end-diastolic pressure (LVP $\left.{ }_{\text {ed }}\right)$ and IVMD, where absolute change was used) were compared 
between the different groups and settings using two-way mixed effects analysis of variance (ANOVA) followed by Bonferroni's multiple comparisons test. We also used these statistical tests to compare the haemodynamic and echocardiographic data collected at baseline with those collected at sacrifice. For comparison of the 4-week data with baseline and sacrifice data, a separate repeated measures one-way ANOVA was performed, followed by Bonferroni's multiple comparisons test. Where appropriate, we applied the Geisser-Greenhouse correction during ANOVA to account for possible violations of the assumption of sphericity. SV and regurgitant fraction in the two groups at sacrifice were compared using an unpaired t-test. $\mathrm{P}$ values $\leq 0.05$ were considered statistically significant. Data are shown as mean \pm standard deviation.

\section{Results}

Figure 1 shows images of a typical animal prior to induction of MR and 20 weeks later. MR (indicated by arrows) was demonstrated by colour Doppler echocardiography, retrograde flux of contrast medium injected into the LV and cardiac magnetic resonance imaging (MRI). After 20 weeks of MR, significant enlargement of the LV cavity was visible, both on the long-axis MRI and short-axis echocardiographic images.

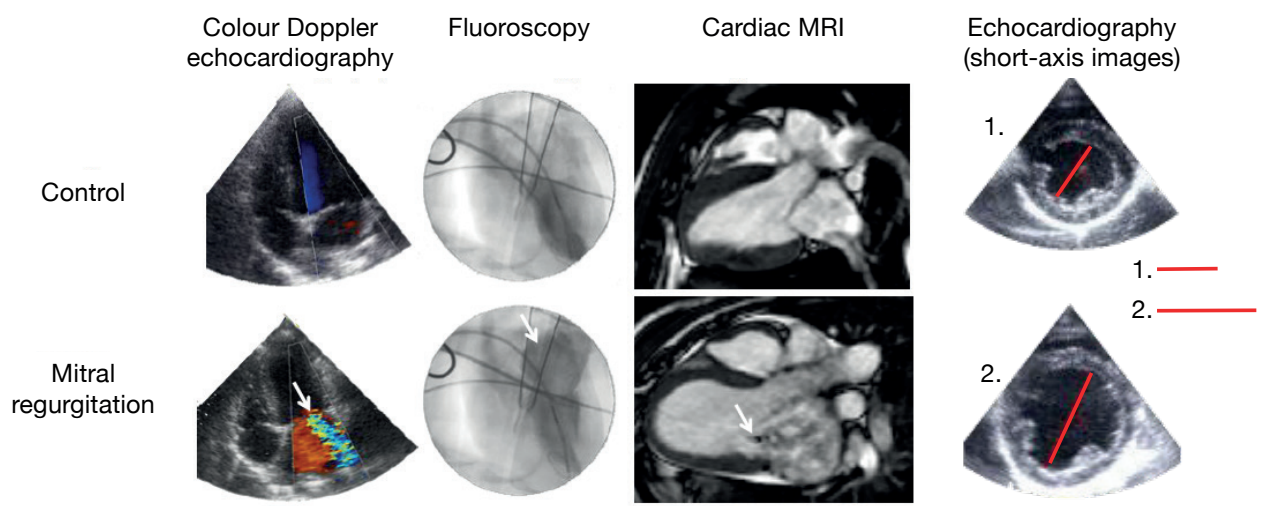

FIGURE 1. Imaging of MR with typical examples of colour Doppler echocardiography, fluoroscopy, cardiac MRI and 2D echocardiography in canine hearts without (upper row) and with MR (lower low). Cardiac MRI and short-axis echocardiography were performed 4 months after inducing MR. Arrows point to the blood regurgitating through the mitral valve. $\mathrm{MR}=$ mitral regurgitation, $\mathrm{MRI}=$ magnetic resonance imaging. Adapted from ${ }^{35}$. 


\section{Haemodynamics and echocardiography over time}

In the LBBB group, inducing LBBB led to a non-significant 50\% increase in LVEDV and a significant $100 \%$ increase in LVESV (Table 1). A significantly larger dilatation was observed in MR+LBBB dogs, especially indicated by a 120\% increase in LVEDV (Table 1).

TABLE 1. Changes in echocardiographic parameters between baseline and medium-term MR+LBBB or LBBB

\begin{tabular}{|c|c|c|c|c|}
\hline & \multicolumn{2}{|c|}{ MR+LBBB $(n=7)$} & \multicolumn{2}{|c|}{ LBBB $(n=11)$} \\
\hline & Baseline & Sacrifice & Baseline & Sacrifice \\
\hline LVEDV (ml) & $52 \pm 8$ & $115 \pm 55^{\star} \#$ & $46 \pm 6$ & $67 \pm 18$ \\
\hline LVESV (ml) & $21 \pm 2$ & $44 \pm 20^{*}$ & $20 \pm 5$ & $37 \pm 13^{*}$ \\
\hline LVEF (\%) & $60 \pm 3$ & $60 \pm 9 \#$ & $57 \pm 8$ & $47 \pm 10^{*}$ \\
\hline Regurgitant fraction (\%) & & $58 \pm 14 \#(n=5)$ & & $-1 \pm 19(n=4)$ \\
\hline Total SV (ml) & & $41 \pm 13 \#(n=5)$ & & $22 \pm 2(n=4)$ \\
\hline Forward SV (ml) & & $17 \pm 5(n=5)$ & & $23 \pm 5(n=4)$ \\
\hline
\end{tabular}

${ }^{*}=p \leq 0.05$ vs. baseline, \# = $p \leq 0.05$ vs. LBBB group at the same time point. Data are shown as mean \pm standard deviation. For abbreviations, see list.

Chronic LBBB resulted in a significant 10\% reduction in LVEF in the LBBB group (Table 1). In contrast, there was no significant change in echocardiographically determined LVEF over time in the MR+LBBB group (Table 1). However, measurements of mitral regurgitant fraction at sacrifice showed severe regurgitation (58 $\pm 14 \%)$ in MR+LBBB animals, whereas there was no significant regurgitation in LBBB animals $(-1 \pm 19 \%$, Table 1). Echocardiographically determined total SV was significantly larger in the MR+LBBB animals than in the LBBB animals (Table 1). However, after subtraction of the regurgitant volume, forward SV tended to be less in the MR+LBBB than in the LBBB animals (Table 1).

Table 2 presents haemodynamic data recorded at various time points under closed-chest conditions. Over time, LVdP/dt ${ }_{\max }$ decreased in both groups. In the MR animals 4 weeks of regurgitation hardly affected $\mathrm{LVdP} / \mathrm{dt}_{\text {max }}$, while chronic $\mathrm{LBBB}$ reduced it to a similar extent in

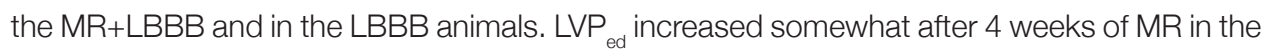
MR+LBBB group, and partly recovered 16 weeks later. 
TABLE 2. Changes in haemodynamic parameters between baseline and medium-term MR+LBBB or LBBB

\begin{tabular}{|c|c|c|c|c|c|}
\hline & \multicolumn{3}{|l|}{$\begin{array}{l}\text { MR+LBBB } \\
(n=8)\end{array}$} & \multicolumn{2}{|l|}{$\begin{array}{l}\text { LBBB } \\
(\mathrm{n}=9)\end{array}$} \\
\hline & BL & 4 weeks & Sacrifice & BL & Sacrifice \\
\hline Heart rate (bpm) & $87 \pm 13$ & $99 \pm 10$ & $97 \pm 18$ & $97 \pm 9$ & $109 \pm 17$ \\
\hline $\mathrm{LVdP} / \mathrm{dt}_{\max }(\mathrm{mmHg} / \mathrm{s})$ & $1953 \pm 239$ & $1798 \pm 305$ & $1263 \pm 359^{\star} \#$ & $1870 \pm 242$ & $1197 \pm 210^{*}$ \\
\hline $\mathrm{LVP}_{\text {ed }}(\mathrm{mmHg})$ & $6 \pm 3$ & $13 \pm 6$ & $10 \pm 10$ & $7 \pm 3$ & $5 \pm 3$ \\
\hline $\operatorname{LVP}_{\text {sys }}(\mathrm{mmHg})$ & $97 \pm 9$ & $97 \pm 13$ & $94 \pm 15$ & $107 \pm 11$ & $93 \pm 12^{*}$ \\
\hline
\end{tabular}

\section{Effects of acute resynchronization therapy}

During the open-chest pacing protocol, baseline values of $\mathrm{LVdP} / \mathrm{dt}_{\max }$ tended to be higher in the MR+LBBB than in the LBBB animals $(1723 \pm 500$ vs. $1210 \pm 277 \mathrm{mmHg} / \mathrm{s}, p=0.10)$. In

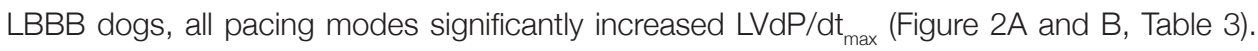
In the MR+LBBB group, BiV ${ }_{\text {best }}$ tended to increase LVdP/dt ${ }_{\max }(1909 \pm 623$ vs. $1723 \pm 500$ $\mathrm{mmHg} / \mathrm{s}$ at baseline, $\mathrm{p}=0.14$, Table 3). No significant differences or changes in $\mathrm{LVdP} / \mathrm{dt}_{\max }$ were observed between pacing modes or animal models (Figure 2A and B, Table 3). LV systolic pressure $\left(\mathrm{LVP}_{\text {sys }}\right)$ did not change significantly during any of the pacing settings in either group (Table 3).

$L_{V} P_{\text {ed }}$ decreased slightly but significantly during BiV ${ }_{\text {best }}$ pacing in the MR+LBBB group and during $L V_{\text {short }}$ pacing in the LBBB group (Table 3). Differences between baseline and $L V_{\text {short }}$ and $\mathrm{LV}_{\text {fusion }}$ pacing in the MR+LBBB group and between baseline and $\mathrm{LV}_{\text {fusion }}$ pacing in the $\mathrm{LBBB}$ group were borderline significant ( $7 \pm 5$ vs. $4 \pm 4 \mathrm{mmHg}, \mathrm{p}=0.07 ; 7 \pm 5$ vs. $4 \pm 4 \mathrm{mmHg}, \mathrm{p}=$ 0.06 and $5 \pm 4$ vs. $4 \pm 4 \mathrm{mmHg}, \mathrm{p}=0.09$, respectively (Table 3)).

When plotting the change in $\mathrm{LVdP}_{\mathrm{d}} \mathrm{dt}_{\max }$ against that of $\mathrm{LVP}_{\text {ed }}$ for all three pacing modes, LBBB animals consistently showed an increase in LVdP/dt max , mostly in combination with a small decrease in LVP ${ }_{\text {ed }}$. The MR+LBBB animals did not show an increase in LVdP/dt ${ }_{\text {max }}$ in a few

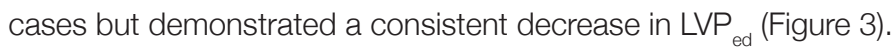

Aortic systolic pressure $\left(\mathrm{AOP}_{\text {sys }}\right)$ and aortic pulse pressure $\left(\mathrm{AOP}_{\text {pulse }}\right)$ were not significantly affected by the various pacing modes in either group (Table 3). $\mathrm{LV}_{\text {short }}$ pacing reduced $\mathrm{RV}$ systolic pressure $\left(R \mathrm{RP}_{\text {sys }}\right.$ ) relative to baseline and to $\mathrm{BiV}_{\text {best }}$ pacing in the LBBB group (Table 3). None of the pacing settings significantly changed maximum rate of RV pressure rise $\left(\mathrm{RVdP} / \mathrm{dt}_{\max }\right)$ compared to baseline (Table 3). 
In both groups IVMD increased significantly from negative values during baseline to positive values during $\mathrm{LV}_{\text {short }}$ pacing (Figure $2 \mathrm{C}$, Table 3). Additionally, in the LBBB dogs, $\mathrm{LV}_{\text {short }}$ pacing led to a more positive IVMD than BiV ${ }_{\text {best }}$ or $\mathrm{LV}_{\text {fusion }}$ pacing (Figure $2 \mathrm{C}$, Table 3).

\section{Discussion}

The main findings of this study are: 1) CRT increases LVdP/dt ${ }_{\max }$ in LBBB and MR+LBBB animals, although the effect is somewhat less pronounced in the latter; 2) in MR+LBBB animals BiV and LV pacing decrease the moderately increased LVP ${ }_{\text {ed }}$; 3 in both groups LV pacing with short AV delay (full capture) or with AV delay aiming at optimal fusion do not provide superior haemodynamic effect compared to BiV pacing. Summarizing, in this animal model of chronic structural MR combined with LBBB, CRT was able to improve cardiac pump function to an extent that is comparable with that in animals with lone chronic LBBB.

\section{The MR+LBBB animal model}

This model results in highly dilated hearts with LBBB. The more than doubling of LVEDV compared to healthy canine hearts (our baseline values) is quite similar to the difference in LVEDV between healthy individuals and heart failure patients ${ }^{7,18}$. Therefore, the MR+LBBB model may better mimic the hearts of CRT candidates than our conventional LBBB model (which shows dilatation to a lesser extent). The clinical similarity was also demonstrated by the fact that many of the MR+LBBB animals showed overt clinical symptoms, like abdominal fluid retention and poor exercise tolerance. Congestion in this model is also evidenced by the increase in $L V P_{\text {ed }}$, at least in the closed-chest situation. Undoubtedly, the creation of MR+LBBB is technically more challenging, because we observed a relatively large number of animals that died prematurely.

Other groups have used other ways to create heart failure, such as tachypacing at about 210 bpm. However, the disadvantage of that approach is that the tachycardia is the dominant factor, reducing the haemodynamic benefit of CRT if this is applied at the same high heart rate $^{19,20}$.

We observed a difference of a little more than $450 \mathrm{mmHg} / \mathrm{s}$ in LVdP/dt max $_{\text {ax }}$ between sacrifice (Table 2) and baseline (start of the pacing protocol, Table 3) in the MR+LBBB dogs. This big difference may be due to the different situations in which the two values were recorded: the "sacrifice" value was recorded when the chest was still closed, while the baseline value was obtained after the chest had been opened and the dogs had been haemodynamically stabilized with intravenous saline and dobutamine as necessary. 
A

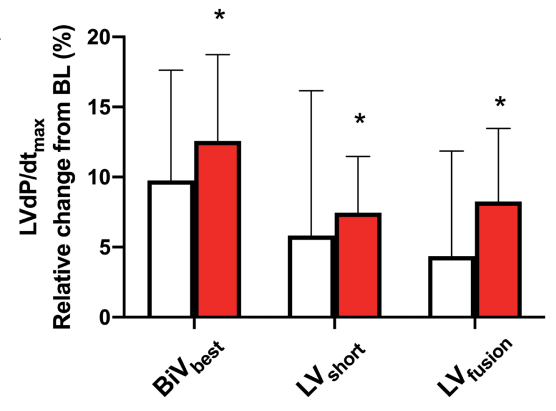

C

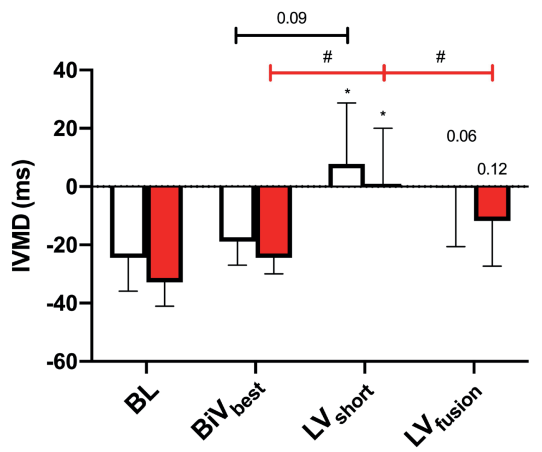

B

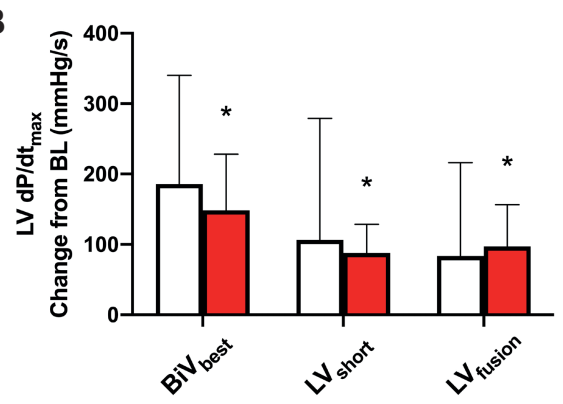

$\square \mathrm{MR}+$ LBBB $\square$ LBBB

FIGURE 2. A. $\mathrm{LVdP}_{\mathrm{dt}} \mathrm{max}_{\max }$ change from baseline in \% during the different pacing modes. B. LVdP/dt ${ }_{\max }$ change from baseline in $\mathrm{mmHg} / \mathrm{s}$ during the different pacing modes. C. IVMD during the different pacing modes. ${ }^{*}=p \leq 0.05$ vs. $B L$ in the respective group, $\#=p \leq 0.05$ vs. LV $_{\text {short }}$ within the LBBB group. For abbreviations, see list.

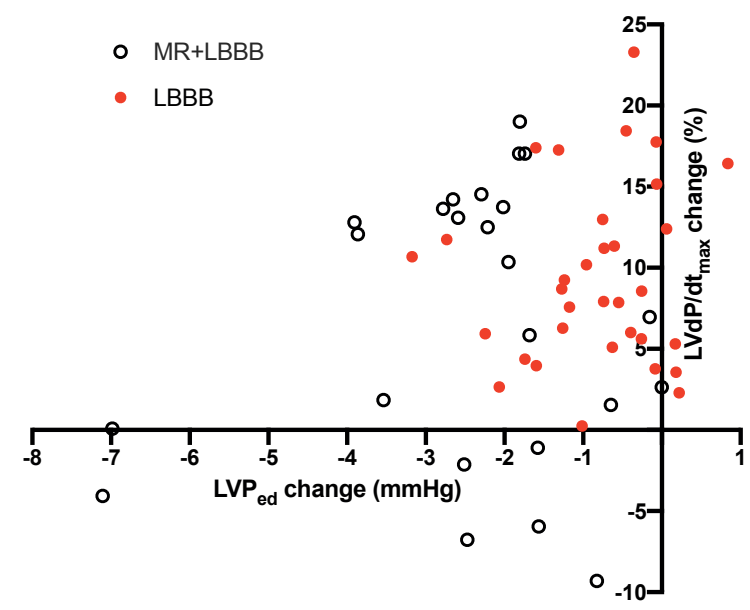

FIGURE 3. Changes from baseline of $\mathrm{LVP}_{\mathrm{ed}}$ in $\mathrm{mmHg}$ and of $\mathrm{LVdP}_{\mathrm{dt}}$ max $\%$ for all studied pacing settings. For abbreviations, see list. 


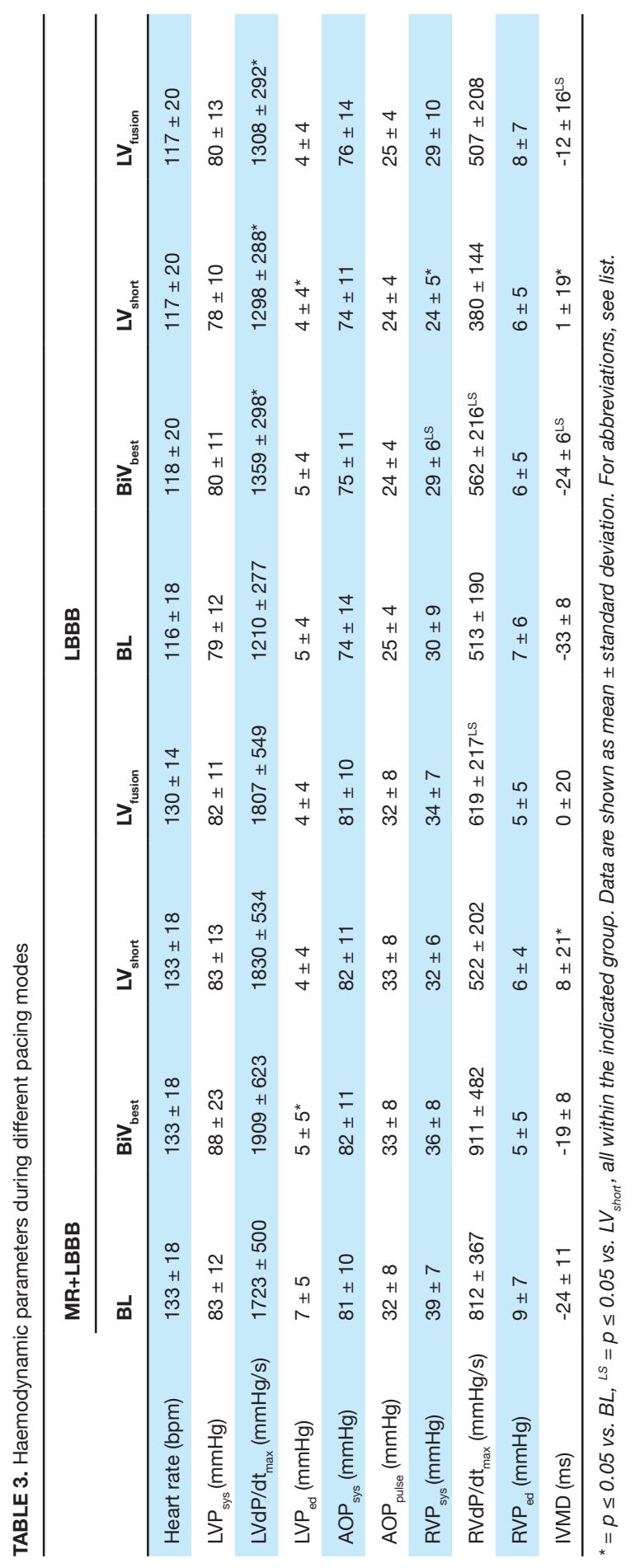




\section{Haemodynamic improvements in the MR+LBBB animals}

Acute increase in $\mathrm{LVdP}_{\mathrm{d}} \mathrm{dt}_{\max }$ due to CRT has been reported in animals ${ }^{21}$ as well as in patients ${ }^{22,23}$ with cardiac dyssynchrony. In our study, we observe similar increases in $\mathrm{LVdP} / \mathrm{dt}_{\max }$ in MR+LBBB and LBBB animals, despite the presence of structural MR in the former. The IVMD value at which we observe a relatively large increase in $\mathrm{LVdP} / \mathrm{dt}_{\max }$ in both animal models is fairly close to the one reported by Verbeek et al. ${ }^{21}$, which also suggests that the presence of MR does not fundamentally change the effect of resynchronization in our animal model. Moreover, while in some cases LV or BiV pacing did not lead to an increase in $\mathrm{LVdP} / \mathrm{dt}_{\text {max }}$ in $\mathrm{MR}+\mathrm{LBBB}$ dogs, the elevated $\mathrm{LVP}_{\text {ed }}$ was reduced in all cases.

It seems plausible that the reduction in LVP $\mathrm{ed}_{\text {is }}$ caused by the better contractility, thereby reducing backward failure. While in haemodynamic studies on CRT the change in $\mathrm{LVdP} / \mathrm{dt}_{\max }$ is the most dominant feature, clinically the reduction in LVP ${ }_{\text {ed }}$ may be at least as relevant. High $L_{V P}$ id linked to congestion and thus symptoms of the patient ${ }^{24}$, meaning that its reduction may be beneficial. Moreover, reducing LVP $_{\text {ed }}$ may lead to a reduction in LV dilatation and thereby to reverse remodelling in the longer term. Several studies have shown that reverse remodelling (reduction of LVEDV > 15\%) is a valuable surrogate endpoint, predicting long-term clinical response 25,26 .

The results of CRT in our animal model of structural MR in combination with LBBB should be compared with care with those from patient studies involving functional MR, be it proportionate or disproportionate. As reported by Spartera et al., CRT may reduce MR in several ways: by increasing valvular closure force through increased $\mathrm{LVdP} / \mathrm{dt}_{\max }$, normalizing mitral valve tethering, improving mitral valve dimensions and improving timing of atrial and ventricular contraction². A few studies suggest that both LV only and BiV pacing may have beneficial effects on MR severity ${ }^{27-29}$. However, it is very unlikely that CRT with either setting has reduced $M R$ in our structural MR+LBBB model. After all, MR due to the ruptured chordae tendineae is unlikely to be influenced by most of the factors mentioned above. In that sense, our MR+LBBB is the worst-case scenario for CRT, where a structural problem is present that it not amenable to CRT. Therefore, the most likely explanation for the better cardiac function (increase in $\mathrm{LVdP}_{\mathrm{dt}}$ max and reduction of $\mathrm{LVP}_{\text {ed }}$ ) is ventricular resynchronization (alleviation of LBBB), thereby increasing ventricular contractility.

The present study only investigated the acute haemodynamic effects of CRT, but a few patient studies showed that CRT may still be beneficial if MR persists ${ }^{4,13}$, although possibly less than when MR is reduced by $\mathrm{CRT}^{4,10}$. However, patients in these cohorts did not have (severe) structural MR (like in our animal model). It would be interesting to see if our findings in the animal model are replicated in patients with structural MR, and, more importantly, whether any short-term improvement also relates to long-term benefit. 
Lastly, whether residual MR after CRT should be treated using mitral valve repair is under debate. The effects of implantation of a MitraClip device in CRT non-responders who also suffered from significant MR appear to be limited ${ }^{30}$. On the other hand, the COAPT trial indicated benefit of a MitraClip device over optimal medical therapy alone on the combined end point of heart failure hospitalizations and all-cause mortality in patients who had previously received $\mathrm{CRT}^{31}$. An important difference is that the former study did not include a control group but predicted survival scores using patient characteristics, while the latter directly compared MitraClip plus optimal medical therapy with optimal medical therapy alone. Moreover, the group sizes were very different (42 vs. 614 patients).

\section{Benefit of BiV and LV only pacing}

Our findings regarding BiV and LV only pacing corroborate outcomes of previous studies in patients and in dogs, showing that both pacing modes have similar haemodynamic benefit in LBBB $^{27,32}$. A chronic study that focused on LV pacing without fusion demonstrated the same outcome $^{33}$. On the other hand, in a study using the adaptive CRT algorithm, optimized LV fusion pacing showed a benefit compared to conventional BiV pacing with regard to clinical outcome $^{34}$. Whether the comparable acute benefits of BiV and LV only pacing we observed would lead to comparable benefit in the long term, or whether LV only fusion pacing is a superior method to resynchronize hearts with structural MR also remains to be investigated.

\section{Limitations}

The number of animals that was studied was small. Since this was an animal model, MR and LBBB were induced in an artificial way. Measurements were done under general anaesthesia and all values were compared to one baseline measurement (rather than alternating between baseline measurements and pacing settings). Forward flow into the aorta was not measured, although it was estimated in a subgroup of animals. Finally, extrapolating results from acute animal studies to long-term clinical effect should be done with care.

\section{Conclusions}

In an animal model of structural MR combined with LBBB, CRT was able to improve cardiac pump function, although the effect was less pronounced than in isolated LBBB. 


\section{References}

1. Packer M, Grayburn PA. Contrasting Effects of Pharmacological, Procedural, and Surgical Interventions on Proportionate and Disproportionate Functional Mitral Regurgitation in Chronic Heart Failure. Circulation 2019;140:779-89.

2. Spartera M, Galderisi M, Mele D, Cameli M, D'Andrea A, Rossi A, et al. Role of cardiac dyssynchrony and resynchronization therapy in functional mitral regurgitation. Eur Heart J Cardiovasc Imaging 2016;17:471-80.

3. Breithardt OA, Sinha AM, Schwammenthal E, Bidaoui N, Markus KU, Franke A, et al. Acute effects of cardiac resynchronization therapy on functional mitral regurgitation in advanced systolic heart failure. J Am Coll Cardiol 2003;41:765-70.

4. Bartko PE, Arfsten H, Heitzinger G, Pavo N, Strunk G, Gwechenberger M, et al. Papillary Muscle Dyssynchrony-Mediated Functional Mitral Regurgitation: Mechanistic Insights and Modulation by Cardiac Resynchronization. JACC Cardiovasc Imaging 2019;12:1728-37.

5. Porciani MC, Macioce R, Demarchi G, Chiostri M, Musilli N, Cappelli F, et al. Effects of cardiac resynchronization therapy on the mechanisms underlying functional mitral regurgitation in congestive heart failure. Eur J Echocardiogr 2006;7:31-9.

6. Salden FCWM, Kutyifa V, Stockburger M, Prinzen FW, Vernooy K. Atrioventricular dromotropathy: evidence for a distinctive entity in heart failure with prolonged PR interval? Europace 2018;20:106777.

7. John Sutton MG St., Plappert T, Abraham WT, Smith AL, DeLurgio DB, Leon AR, et al. Effect of cardiac resynchronization therapy on left ventricular size and function in chronic heart failure. Circulation 2003;107:1985-90.

8. Mihos CG, Santana O, Yucel E, Capoulade R, Upadhyay GA, Orencole MP, et al. The effects of cardiac resynchronization therapy on left ventricular and mitral valve geometry and secondary mitral regurgitation in patients with left bundle branch block. Echocardiography 2019;36:1450-8.

9. Bijl P van der, Khidir M, Ajmone Marsan N, Delgado V, Leon MB, Stone GW, et al. Effect of Functional Mitral Regurgitation on Outcome in Patients Receiving Cardiac Resynchronization Therapy for Heart Failure. Am J Cardiol 2019;123:75-83.

10. Verhaert D, Popović ZB, De S, Puntawangkoon C, Wolski K, Wilkoff BL, et al. Impact of mitral regurgitation on reverse remodeling and outcome in patients undergoing cardiac resynchronization therapy. Circ Cardiovasc Imaging 2012;5:21-6.

11. Onishi T, Onishi T, Marek JJ, Ahmed M, Haberman SC, Oyenuga O, et al. Mechanistic features associated with improvement in mitral regurgitation after cardiac resynchronization therapy and their relation to long-term patient outcome. Circ Hear Fail 2013;6:685-93.

12. Cleland J, Freemantle N, Ghio S, Fruhwald F, Shankar A, Marijanowski M, et al. Predicting the LongTerm Effects of Cardiac Resynchronization Therapy on Mortality From Baseline Variables and the Early Response. A Report From the CARE-HF (Cardiac Resynchronization in Heart Failure) Trial. $J$ Am Coll Cardiol 2008;52:438-45. 
13. John Sutton M St., Ghio S, Plappert T, Tavazzi L, Scelsi L, Daubert C, et al. Cardiac resynchronization induces major structural and functional reverse remodeling in patients with New York heart association class I/II heart failure. Circulation 2009;120:1858-65.

14. Biase L Di, Auricchio A, Mohanty P, Bai R, Kautzner J, Pieragnoli P, et al. Impact of cardiac resynchronization therapy on the severity of mitral regurgitation. Europace 2011;13:829-38.

15. Brignole M, Auricchio A, Baron-Esquivias G, Bordachar P, Boriani G, Breithardt O-A, et al. 2013 ESC Guidelines on cardiac pacing and cardiac resynchronization therapy. Eur Heart J 2013;34:2281329.

16. Verbeek XAAM, Vernooy K, Peschar M, Nagel T Van Der, Hunnik A Van, Prinzen FW. Quantification of interventricular asynchrony during LBBB and ventricular pacing. Am J Physiol - Hear Circ Physiol 2002;283:1370-8.

17. VerbeekXAAM, Auricchio A, YuY, Ding J, Pochet T, Vernooy K, et al. Tailoring cardiac resynchronization therapy using interventricular asynchrony. Validation of a simple model. Am J Physiol - Hear Circ Physiol 2006;290:968-77.

18. Maceira AM, Prasad SK, Khan M, Pennell DJ. Normalized left ventricular systolic and diastolic function by steady state free precession cardiovascular magnetic resonance. J Cardiovasc Magn Reson 2006;8:417-26.

19. Vernooy K, Cornelussen RNM, Verbeek XAAM, Vanagt WYR, Hunnik A Van, Kuiper M, et al. Cardiac resynchronization therapy cures dyssynchronopathy in canine left bundle-branch block hearts. Eur Heart J 2007;28:2148-55.

20. Chakir K, Daya SK, Tunin RS, Helm RH, Byrne MJ, Dimaano VL, et al. Reversal of global apoptosis and regional stress kinase activation by cardiac resynchronization. Circulation 2008;117:1369-77.

21. Verbeek XAAM, Vernooy K, Peschar M, Cornelussen RNM, Prinzen FW. Intra-ventricular resynchronization for optimal left ventricular function during pacing in experimental left bundle branch block. J Am Coll Cardiol 2003;42:558-67.

22. Gold MR, Niazi I, Giudici M, Leman RB, Sturdivant JL, Kim MH, et al. A prospective, randomized comparison of the acute hemodynamic effects of biventricular and left ventricular pacing with cardiac resynchronization therapy. Hear Rhythm 2011;8:685-91.

23. Nelson GS, Berger RD, Fetics BJ, Talbot M, Spinelli JC, Hare JM, et al. Left ventricular or biventricular pacing improves cardiac function at diminished energy cost in patients with dilated cardiomyopathy and left bundle-branch block. Circulation 2000;102:3053-9.

24. Pinamonti $B$, Lenarda $A D i$, Sinagra $G$, Camerini $F$. Restrictive left ventricular filling pattern in dilated cardiomyopathy assessed by doppler echocardiography: Clinical, echocardiographic and hemodynamic correlations and prognostic implications. J Am Coll Cardiol 1993;22:808-15.

25. Solomon SD, Foster E, Bourgoun M, Shah A, Viloria E, Brown MW, et al. Effect of cardiac resynchronization therapy on reverse remodeling and relation to outcome: Multicenter automatic defibrillator implantation trial: Cardiac resynchronization therapy. Circulation 2010;122:985-92.

26. Bleeker GB, Bax JJ, Fung JWH, Wall EE Van Der, Zhang Q, Schalij MJ, et al. Clinical versus echocardiographic parameters to assess response to cardiac resynchronization therapy. Am J Cardiol 2006; $97: 260-3$. 
27. Bordachar P, Lafitte S, Reuter S, Garrigue S, Sanders P, Roudaut R, et al. Biventricular pacing and left ventricular pacing in heart failure: Similar hemodynamic improvement despite marked electromechanical differences. J Cardiovasc Electrophysiol 2004;15:1342-7.

28. Rao RK, Kumar UN, Schafer J, Viloria E, Lurgio D De, Foster E. Reduced ventricular volumes and improved systolic function with cardiac resynchronization therapy: A randomized trial comparing simultaneous biventricular pacing, sequential biventricular pacing, and left ventricular pacing. Circulation 2007;115:2136-44.

29. Vinereanu D, Bleasdale R, Turner M, Frenneaux MP, Fraser AG. Comparison of left ventricularbiventricular pacing on ventricular synchrony, mitral regurgitation, and global left ventricular function in patients with severe chronic heart failure. Am J Cardiol 2004;94:519-21.

30. Seifert M, Schau T, Schoepp M, Arya A, Neuss M, Butter C. MitraClip in CRT non-responders with severe mitral regurgitation. Int J Cardiol 2014;177:79-85.

31. Stone GW, Lindenfeld JA, Abraham WT, Kar S, Lim DS, Mishell JM, et al. Transcatheter mitral-valve repair in patients with heart failure. N Engl J Med 2018;379:2307-18.

32. Lumens J, Ploux S, Strik M, Gorcsan J, Cochet H, Derval N, et al. Comparative electromechanical and hemodynamic effects of left ventricular and biventricular pacing in dyssynchronous heart failure: Electrical resynchronization versus left-right ventricular interaction. J Am Coll Cardiol 2013;62:2395403.

33. Thibault B, Ducharme A, Harel F, White M, O'Meara E, Guertin M-C, et al. Left ventricular versus simultaneous biventricular pacing in patients with heart failure and a QRS complex $\geq 120$ milliseconds. Circulation 2011;124:2874-81.

34. Birnie D, Lemke B, Aonuma K, Krum H, Lee KLF, Gasparini M, et al. Clinical outcomes with synchronized left ventricular pacing: Analysis of the adaptive CRT trial. Hear Rhythm 2013;10:136874.

35. Van Middendorp, LB. (2015) Cardiac dyssynchrony: structural, functional, transcriptional and pharmacological aspects. Doctoral thesis, Maastricht University, Maastricht. 


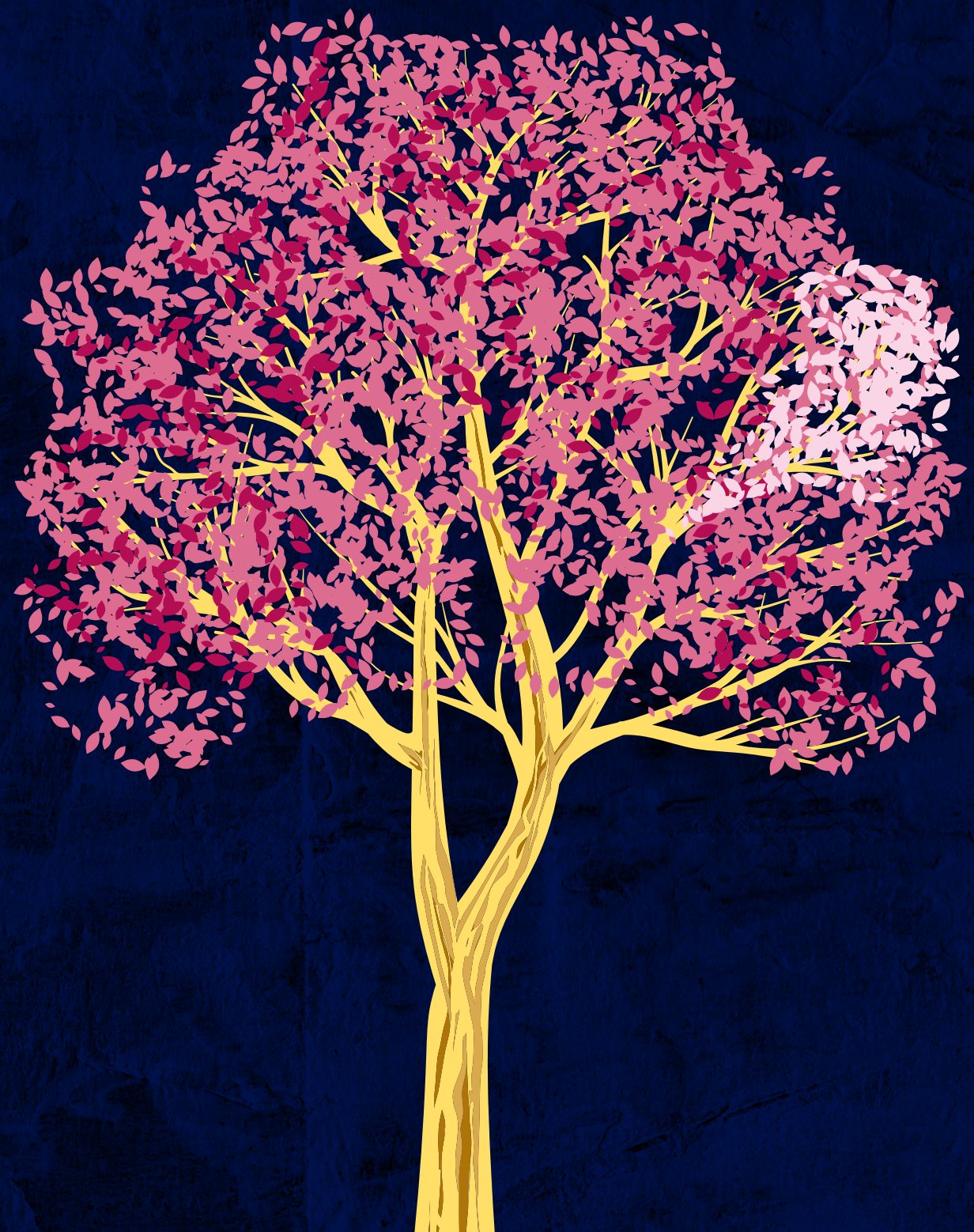




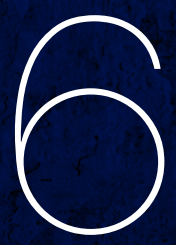

\section{Exploring the cause of conduction delays in patients with repaired Tetralogy of Fallot}

Nienke J. Verzaal' ${ }^{1}$, Stéphane Massé², Eugene Downar², Kumaraswamy Nanthakumar², Tammo Delhaas ${ }^{3}$ and Frits W. Prinzen ${ }^{1}$

\footnotetext{
'Department of Physiology, Maastricht University, Maastricht, The Netherlands

${ }^{2}$ The Hull Family Cardiac Fibrillation Management Laboratory, Toronto General Hospital, Toronto, Canada

${ }^{3}$ Department of Biomedical Engineering, Maastricht University, Maastricht, The Netherlands
}

Europace 2021;23:i105-12 


\section{Abstract}

Aims Cardiac dyssynchrony in patients with repaired Tetralogy of Fallot (rToF) has been attributed to right bundle branch block (RBBB), fibrosis and/or the patches that are inserted during repair surgery. We aimed to investigate the basis of abnormal activation in rToF patients by mapping the electrical activation sequence during sinus rhythm (SR) and right ventricular (RV) pacing.

Methods A total of 17 patients were studied (13 with rToF, 2 with left bundle branch block (LBBB) and 2 without RBBB or LBBB (non-BBB)) during medically indicated cardiac surgery. During SR and RV pacing, measurements were performed using 112-electrode RV endocardial balloons (rToF only) and biventricular epicardial sock arrays (4 of the rToF and all non-rToF patients).

Results During SR, functional lines of block occurred in 5 rToF patients while RV pacing caused functional blocks in 4 rToF patients. The line of block persisted during both SR and RV pacing in only 2 out of 13 rToF patients. Compared to SR, RV pacing increased dispersion of septal activation, but not dispersion of endocardial and epicardial activation of the RV free wall. During pacing, RV and left ventricular activation dispersion of rToF patients were comparable to those of the non-rToF patients.

Conclusion The results of the present study indicate that the delayed activation in the RV of rToF patients is predominantly due to block(s) in the Purkinje system and that conduction in $\mathrm{RV}$ tissue is fairly normal. 


\section{Introduction}

Tetralogy of Fallot (ToF), the most common congenital cyanotic cardiac disease, is characterized by a ventricular septal defect (VSD), overriding aorta, pulmonary stenosis and concomitant hypertrophy of the right ventricle (RV) 1 . During surgery, which is usually performed at a very young age, the VSD is closed and the pulmonary stenosis is alleviated. While surgery has a high chance of success to restore anatomy, it often results in right bundle branch block (RBBB), post-operative pulmonary insufficiency and residual stenosis. These three conditions are potentially harmful for proper cardiac function and may negatively affect the prognosis of patients with repaired Tetralogy of Fallot (rToF).

Patients with rToF often display dyssynchrony, and larger dyssynchrony is correlated to lower exercise capacity and increased risk of ventricular arrhythmias ${ }^{2}$.

An obvious candidate cause of this dyssynchrony is damage to part of the rapid conduction system, in particular the right bundle branch ${ }^{3}$. Electrophysiological studies performed during rToF surgery showed RBBB ${ }^{3,4}$ and also left anterior hemiblock has been found post-surgery ${ }^{5}$.

However, structural abnormalities (like fibrosis) due to longer-lasting RV overload and related $\mathrm{RV}$ dilation, surgical scar and the patch used to relieve pulmonary stenosis may cause or aggravate the dyssynchrony, ${ }^{6,7}$.

The present study aims to assess if, and to what extent, abnormal activation in rToF hearts is due to structural tissue abnormalities, by examining the presence of conduction block and dispersion of activation using detailed endo- and epicardial mapping. Measurements during sinus rhythm (SR) and RV pacing allowed us to distinguish between the conductive effects of the His-Purkinje system and myocardial tissue properties and also between structural and functional blocks. Data from rToF patients was compared with a small group of non-rToF patients.

\section{Materials and Methods}

\section{Study population and mapping array}

Studies were performed at Toronto General Hospital, between 1995 and 2008. This study was approved by the University Health Network Ethics Review Board. All patients provided informed consent prior to inclusion. 
During medically indicated cardiac surgery, detailed RV mapping was performed in 13 rToF patients (4 women, 9 men, age $37 \pm 8$ (mean \pm standard deviation)), using an RV cavityshaped endocardial balloon array consisting of 112 bipolar electrodes (Figure 1A). Additionally, in 4 of these patients, an epicardial sock array with 112 bipolar electrodes (Figure 1B) was used to map both left ventricular (LV) and RV activation. The latter array was also used in 2 patients with left bundle branch block (LBBB)(1 woman, 1 man, age 61) and 2 patients without either RBBB or LBBB (non-BBB, both men, age 64).
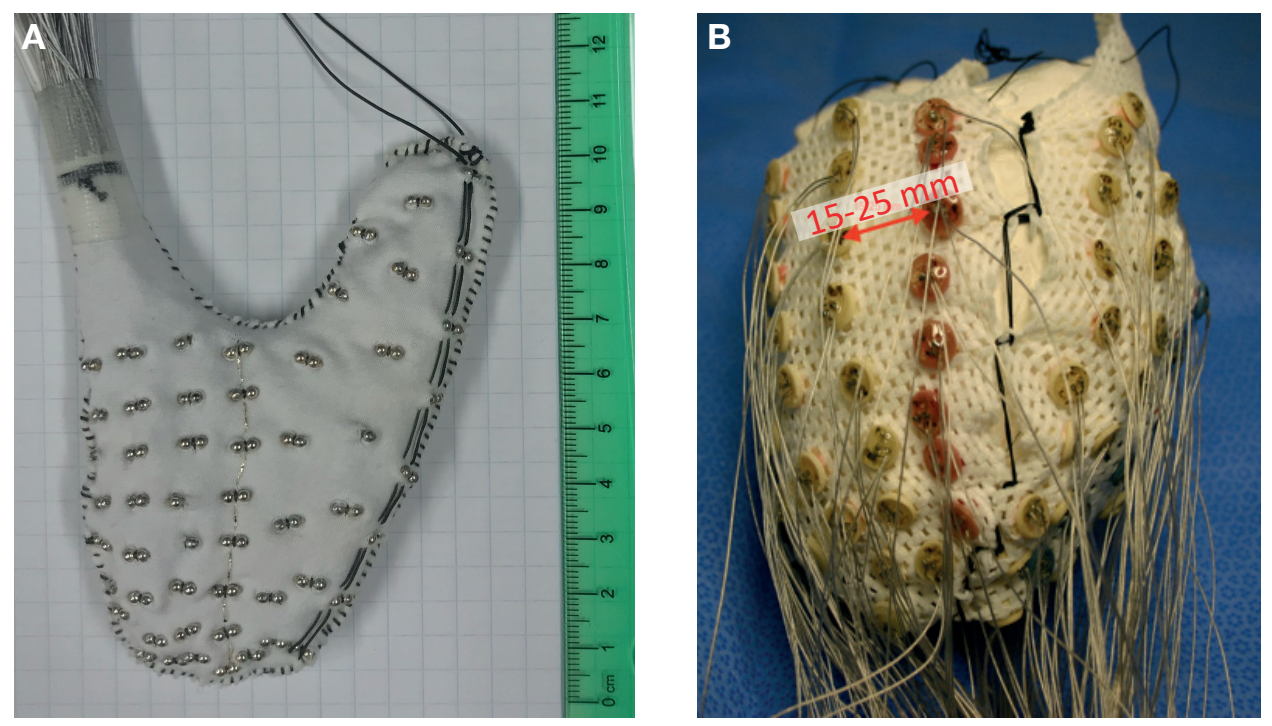

FIGURE 1. A. The endocardial right ventricular (RV) balloon array. The thumb-like structure on the right side was positioned inside the RV outflow tract. B. The epicardial sock array.

The arrays have been described in previous publications, that already addressed some aspects of cardiac activation during SR in some of the patients described in this paper ${ }^{8,9}$. Briefly, the 112 electrode pairs on each of the arrays were divided over 14 vertical splines of 8 pairs each. For all 112 channels, unipolar (sampling rate $1000 \mathrm{~Hz}$, filtering $0.5-200 \mathrm{~Hz}$ ) and bipolar signals (sampling rate $2000 \mathrm{~Hz}$, filtering $28-750 \mathrm{~Hz}$ ) were recorded simultaneously. The electrodes were assigned a region (details in legends to Figures 2 and 3).

In the 4 patients where both arrays were used, the sites of earliest activation on both sock and balloon were overlaid during analysis of paced maps, to ensure proper assignment of the electrodes on the sock to either LV or RV on the epicardium. 
In order to increase the number of observations in LBBB hearts, relevant data from literature describing 4 patients who underwent electrocardiographic (ECG) imaging during both SR and $\mathrm{RV}$ pacing were included ${ }^{10}$.

\section{Data acquisition and analysis}

Mapping was performed after the initiation of cardiopulmonary bypass. Unipolar and bipolar electrograms were recorded, using an epicardial needle stitched onto the base of the heart as a reference. Measurements were performed during SR and during RV pacing from an epicardial site.

Unipolar and bipolar electrograms were analysed using custom-written software to determine local activation times, using the onset of the QRS complex or the middle of the pacing spike as time reference.

Using custom-written MATLAB software (MathWorks, Natick, MA), activation patterns were visualized in activation maps, and several parameters were calculated:

- Average activation time (ms) per region: allowing gross comparison of the order of activation between regions

- $\quad$ Regional activation dispersion (ms): the difference between the 5 and 95\% percentile of activation times in each region

- Total activation time (ms): the latest activation time of the epicardial sock array

\section{Statistical analysis}

Statistical analysis was performed using GraphPad Prism 8 (GraphPad Software, San Diego, USA). Differences in activation times and regional activation dispersions per region of the balloon were assessed using a Friedman test, followed by a Dunn's multiple comparison test. Activation dispersions of the whole balloon during SR and pacing were compared using a Wilcoxon matched pairs signed rank test. A p-value $\leq 0.05$ was considered to be significant. Due to the small number of patients who were examined using the sock array, no statistical analysis was performed for this data. 


\section{Results}

\section{Endo- and epicardial activation maps}

For the majority of the rToF patients, endocardial RV activation (measured on the balloon array) started on the septum and then spread to the RV free wall (RVFW) and RV outflow tract (RVOT) (Figure 2A and C). Upon RV pacing, the RVFW became activated earlier than the septum and the RVOT (Figure 2B and D).

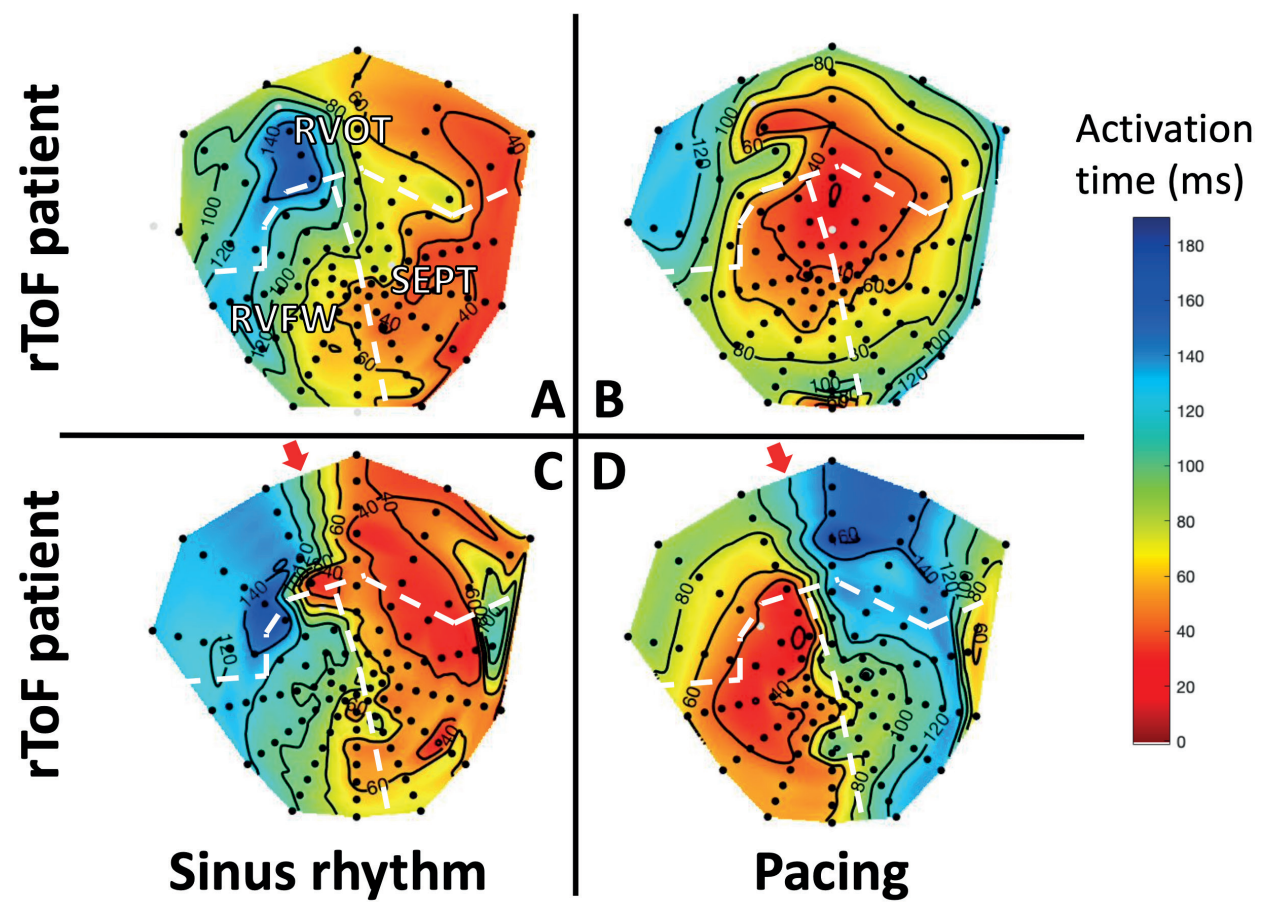

FIGURE 2. Activation maps of the right ventricular (RV) balloon for two patients with repaired Tetralogy of Fallot (rToF), during sinus rhythm $(A, C)$ and pacing $(B, D)$. The white lines indicate the borders between the different areas: RV free wall (RVFW), septum (SEPT) and RV outflow tract (RVOT). The red arrows point to the structural line of block. Grey dots denote electrodes with poor signal quality, omitted from analysis.

Both during SR and during pacing functional blocks occurred. The locations of these blocks varied considerably between patients and between activation modes. In 7 rToF patients, activation progressed fairly regularly during SR, while 5 patients had a functional block that 
disappeared during pacing (example of the latter in Figures $2 A \& B$ ). These functional blocks occurred in the RVOT $(n=3)$, septum $(n=2)$, RVFW $(n=1)$ or in the septum/RVOT border area $(n=1)$. (Note that a patient can have more than one block.)

In the remaining patient and in one of the patients with functional block, a structural block was present (example in Figures 2C \& D). During pacing, functional blocks appeared in 4 patients, in the RVOT ( $n=3)$, septum ( $n=1)$ and in the septum/RVFW border area $(n=1)$. During $S R$, no blocks were present in 2 out of these 4 patients.

During SR, the RVFW was the main late activated area in two of the four rToF patients mapped epicardially, (example in Figure 3A, paced map in Figure 3B), whereas in one patient this was the antero-basal area of both RV and LV (Figure 3C) and in another patient the LV.

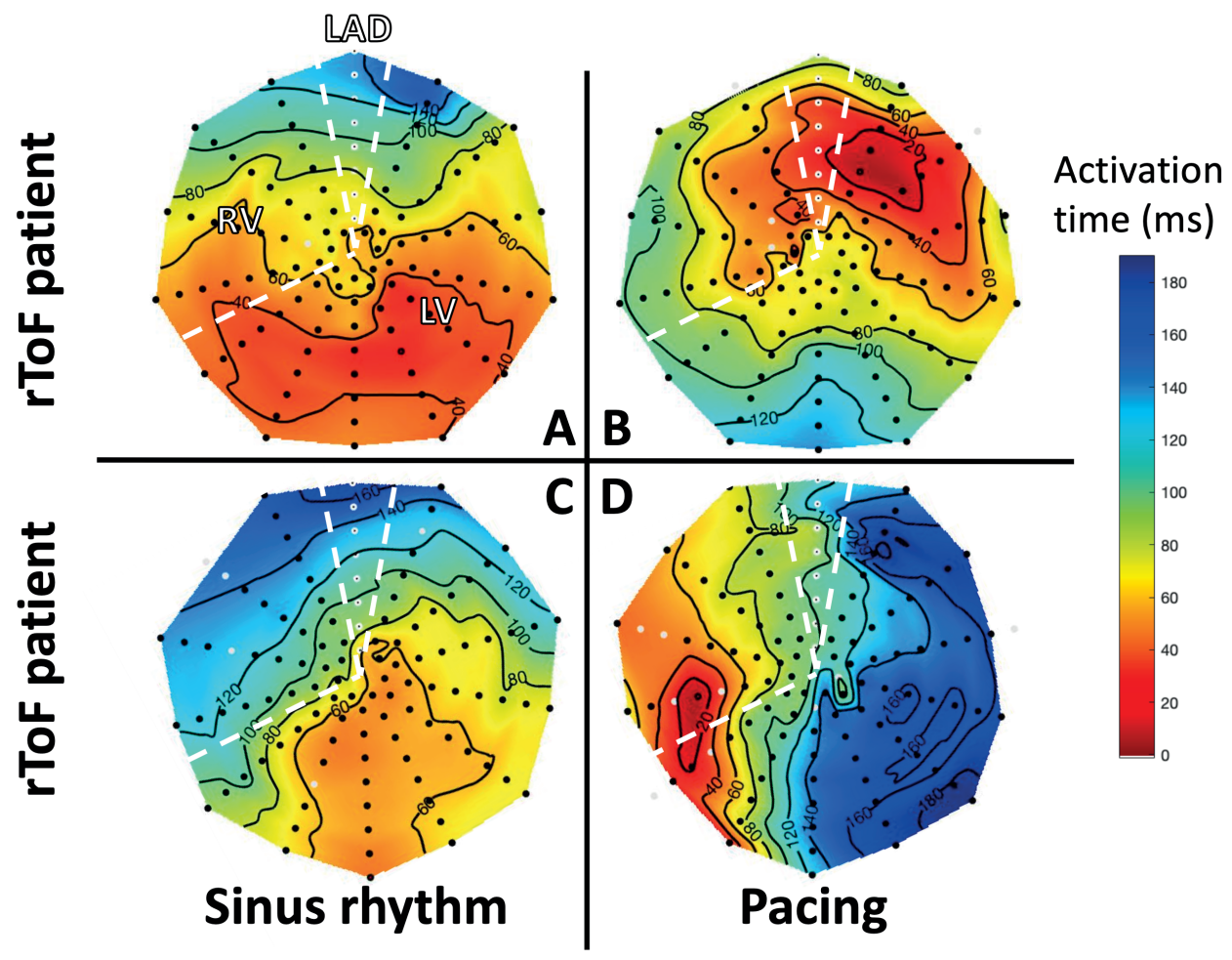

FIGURE 3. Activation maps of the epicardial sock for two patients with repaired Tetralogy of Fallot (rToF), during sinus rhythm $(A, C)$ and pacing $(B, D)$. One spline is aligned with the left anterior descending artery (LAD), the white lines indicate the borders between the different areas: left ventricle (LV), right ventricle (RV) and LAD. Grey dots denote electrodes with poor signal quality, omitted from analysis. 
For the non-BBB patients, during SR the latest activated area was found in the LV (1 patient, Figure $4 \mathrm{~A}$ ) or the RV (1 patient), while in both LBBB patients, the latest activation occurred in the LV (Figure 4C). In all patient groups, RV pacing created early activation near the pacing electrode and spread of activation towards the opposite wall.

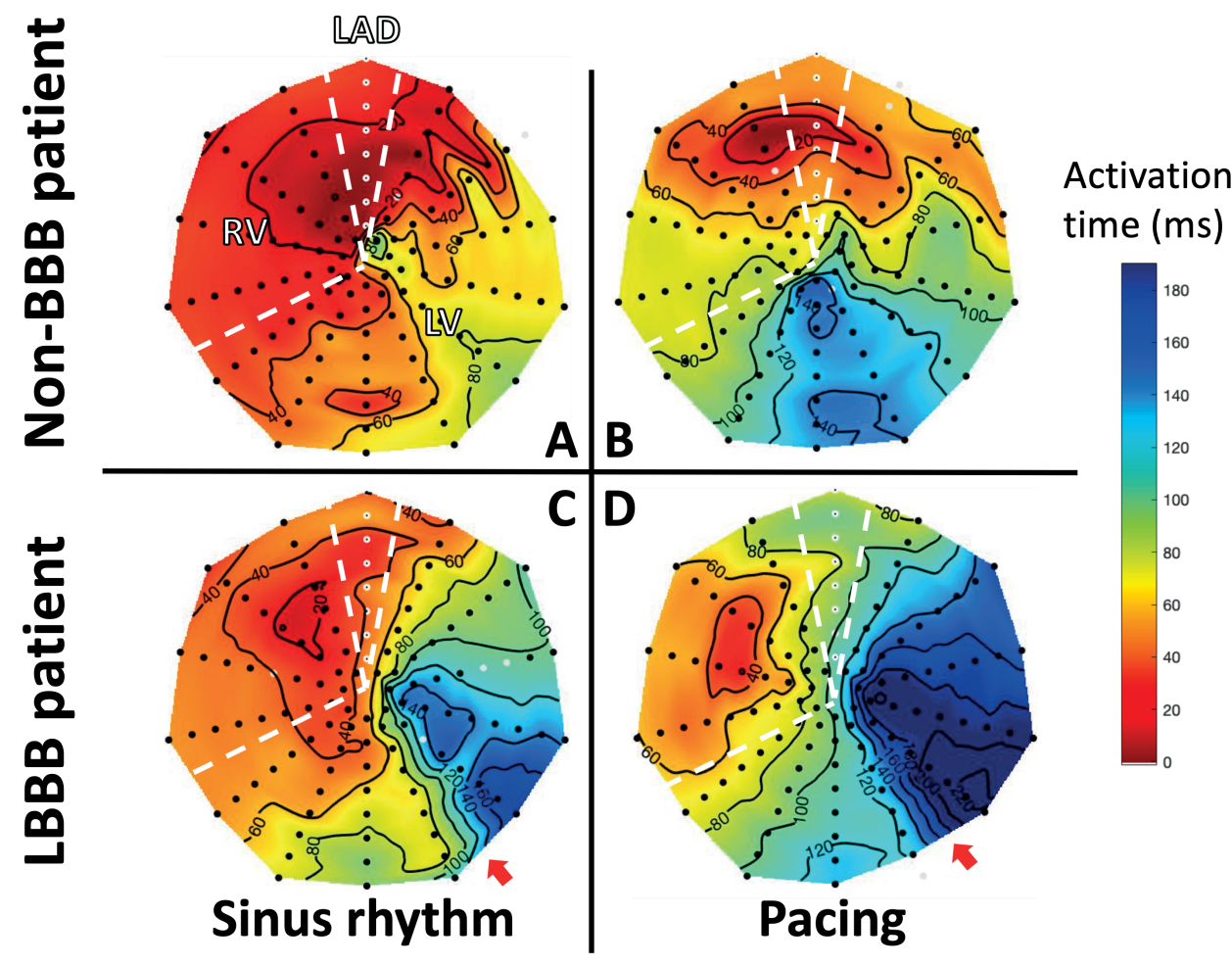

FIGURE 4. Activation maps of the epicardial sock for one patient without either right or left bundle branch block (nonBBB)(A and $B$ ) and one patient with left bundle branch block (LBBB)(C and D) during sinus rhythm (A, C) and pacing $(B, D)$. The red arrows point to the structural line of block. Grey dots denote electrodes with poor signal quality, omitted from analysis. Area abbreviations as in Figure 3.

No epicardial lines of block were observed during SR in any of the four rToF patients, whereas during RV pacing, a line of block occurred in the LV in one patient (Figure 3D). In the non-BBB patients, no lines of block were observed during either SR or RV pacing. In one of the LBBB patients, a persistent line of block occurred in the LV (Figure 4C and D), while in the other LBBB patient, a line of block was observed during LV pacing. 
In summary, functional blocks, that (dis)appeared during ventricular pacing, were found in 8 out of 13 rToF patients, while only 2 had a structural conduction block. One of the two LBBB patients had a functional block and the other patient had a structural block.

\section{Quantification of cardiac activation patterns}

Data from all rToF patients show that during SR the RVFW $(92 \pm 10 \mathrm{~ms}$, mean \pm standard deviation, $p=0.001$ ) and RVOT ( $85 \pm 19 \mathrm{~ms}, \mathrm{p}=0.068$ ) are later activated than the septum ( $57 \pm 11 \mathrm{~ms}$ ), whereas RV pacing results in earlier activation of the RVFW (57 $\pm 9 \mathrm{~ms} ; \mathrm{p}=0.001$ vs. SR) and later activation of the septum (94 $\pm 18 \mathrm{~ms} ; \mathrm{p}<0.001 \mathrm{vs}$. RVFW) and RVOT (114 \pm 22 ms; $p<0.0001$, Figure 5A).

Average epicardial LV activation time of rToF patients (67 (55 - 83) ms, mean and range) and non-BBB patients (51 and $77 \mathrm{~ms}$ ) was comparable, whereas it was larger in the LBBB patients (114 (88 - 144) ms) than in all rToF and non-BBB patients (Figure 5B). Pacing increased the average epicardial LV activation time for all but one patient (rToF 101 (73 - 132) ms; non-BBB 98 and 183 ms; LBBB 133 (121 - 149) ms). Mean increase in rToF was 34 ms, 77 ms in nonBBB and $19 \mathrm{~ms}$ in LBBB (Figure 5B). On average, mean RV activation time was larger in rToF patients (95 (73 - 117) $\mathrm{ms}$ ) than in the non-BBB (21 and $113 \mathrm{~ms})$ and the LBBB patients (46 (29 - 84) ms; Figure 5C). During pacing, average RV activation occurred consistently earlier in the rToF patients while it became later in the other patients (rToF 65 (53 - 71) ms, non-BBB 61 and $150 \mathrm{~ms}$, LBBB 85 (36 - 188) ms). The mean change in activation times was -30 ms (rToF), +39 ms (non-BBB) and +38 ms (LBBB); Figure 5C).

Total epicardial activation times were comparable between rToF patients (161 ms (151-170) mean and range), LBBB patients (174 ms for both) and one of the non-BBB patients (95 and $150 \mathrm{~ms}$ ) during SR. During ventricular pacing, total activation time increased in the non-BBB patients (to 146 and $242 \mathrm{~ms}$ ) and LBBB patients (to 220 and $224 \mathrm{~ms}$ ), whereas it did not consistently change in the rToF patients (161 ms (133-188)).

\section{Activation dispersion}

The rToF patients had a total activation dispersion within the RV balloon of 93 ms (range 49$114 \mathrm{~ms}$ ) during SR. Activation dispersion did not differ between septum (57 $\pm 27 \mathrm{~ms}$, mean \pm standard deviation) and RVFW during SR (61 $\pm 15 \mathrm{~ms})$, but during pacing, septal activation dispersion increased significantly (to $78 \pm 16 \mathrm{~ms}$; $p=0.038$ vs. septum during SR and $p=$ 0.027 vs. RVFW during pacing; Figure 5D). This increase is likely to be largely responsible for the significant increase in total activation dispersion during RV pacing (to $114 \mathrm{~ms}$, (83-141 ms), $p=0.006)$. 
During SR, epicardial LV activation dispersion was smallest in the non-BBB patients (35 and $62 \mathrm{~ms}$ ), intermediate in 3 out of 4 rToF patients (82 (64 - 128) ms, mean and range) and largest in the LBBB patients (110 and $132 \mathrm{~ms}$; Figure 5E). During RV pacing, LV activation dispersion increased in all but one rToF and one LBBB patient so that activation dispersion became comparable between the three patient groups (rToF 103 (65 - 125) ms, non-BBB 92 and 131 ms, LBBB 121 and 140 ms; Figure 5E).

A

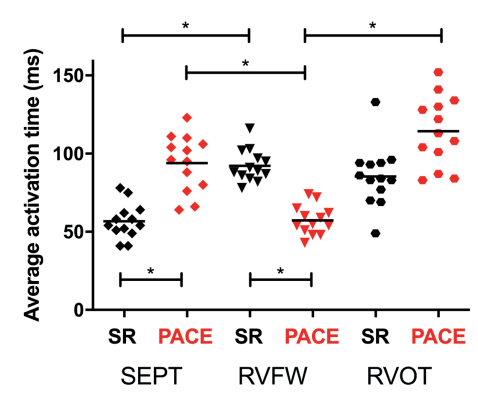

B

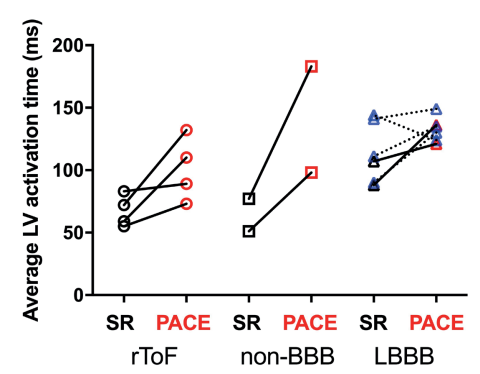

C

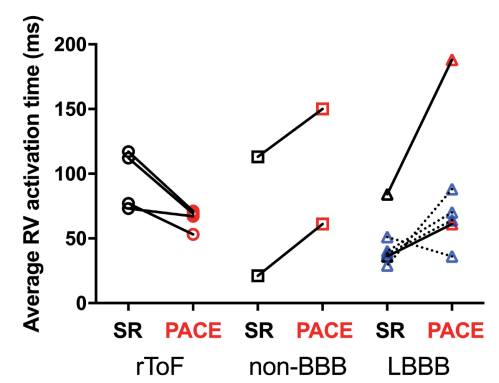

D

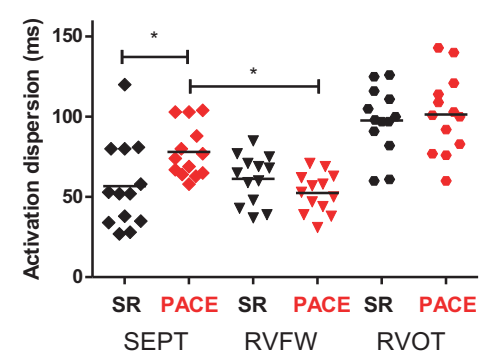

E

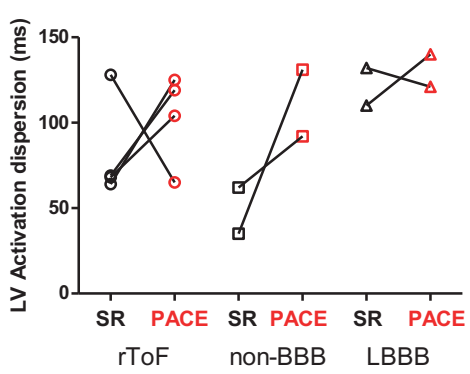

F

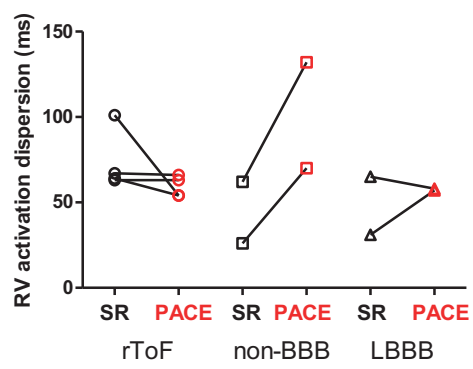

FIGURE 5. Average activation times in different zones of the endocardial RV balloon array (A) and at the LV (B) and RV epicardium (C). Dispersion of activation times in different zones of the endocardial RV balloon array (D) and at the LV (E) and RV epicardium (F). Black symbols denote sinus rhythm (SR), red symbols denote pacing (PACE), other abbreviations as in Figures 2, 3 and 4. Blue triangles in panels $\mathbf{B}$ and $\mathbf{C}$ denote data obtained from a study using electrocardiographic imaging ${ }^{10}$. Horizontal lines in panels $\mathbf{A}$ and $\mathbf{D}$ represent the mean values. ${ }^{*}=p \leq 0.05$ between the indicated values. 
On average, epicardial RV activation dispersion was larger in the rToF patients (74 (63 - 101) $\mathrm{ms}$ ) than in the other patients (non-BBB 26 and $62 \mathrm{~ms}$, LBBB 31 and $65 \mathrm{~ms}$; Figure 5F). This dispersion decreased upon RV pacing in 2 of the 4 rToF patients, while increasing in the non-BBB patients and one LBBB patient. Importantly, this made RV dispersion of activation comparable between the patient groups (rToF 59 (54 - 66) ms; non-BBB 70 and 132 ms, LBBB 57 and $58 \mathrm{~ms}$ ) and one non-BBB patient showed the largest activation dispersion (Figure 5F).

\section{Discussion}

The main findings of the present study are that in rToF patients 1) structural lines of block in the RV are rare; 2) dispersion of activation in both endo- and epicardium of the RVFW is virtually unchanged between SR and RV pacing and 3) during RV pacing, epicardial activation dispersion in both RV and LV is comparable with that in non-rToF patients. These findings indicate that conduction properties of the working myocardium are fairly well-preserved and that the delayed activation in the RV of rToF patients is likely in large part due to blocks in the Purkinje system. The latter has important implications for the potential application of cardiac resynchronization therapy (CRT) in these patients. The persistence of lines of block in only 2 out of the 13 patients indicates that structural regions of block were present in only a minority of rToF patients. Functional blocks during SR were present in about $40 \%$ of the patients, and during pacing in approximately $30 \%$.

\section{Lines of block in rToF patients}

In the majority of rToF patients (11/13), there were no lines of structural block that persisted during both SR and pacing. We consider this finding to be evidence for the idea that a defect of (part of) the rapid conduction system, in particular the right bundle branch, is the primary cause of the abnormal conduction in rToF hearts. Indeed, RBBB morphology of the QRS complex is a common finding after surgical repair of $\mathrm{ToF}^{6,11}$, which has been attributed to damage to the right bundle branch due to VSD closure, RV ventriculotomy or resection of stenotic tissue ${ }^{3,4}$.

The evidence of lines of functional block may, however, point to some conduction problems at the tissue level, such as fibrosis and electrical remodelling. In 8 of the $13 \mathrm{rToF}$ patients, functional lines of block were visible endocardially during SR and/or pacing. The (dis)appearance of lines of block may be explained by the fact that collagen can affect conduction in an anisotropic manner, meaning that activation of the tissue in one direction will lead to conduction block, while conduction in another direction is not negatively influenced ${ }^{7,12}$. Since the functional blocks often occurred in the RVOT (in 5/8 patients who had functional block during either SR or pacing), we may speculate that surgical scars are (partially) responsible for these blocks. 
Additionally, since fibrotic tissue has been found outside of the patches in RVOT and VSD both in rToF patients ${ }^{13}$ and in animal models of $\mathrm{rToF}^{14,15}$, fibrosis may also have played a role in functional blocks outside of the RVOT. However, delays in regions with fibrosis are also governed by the length of fibrotic strands, with short strands hardly affecting conduction ${ }^{7}$. It is therefore possible that fibrotic tissue did not automatically cause conduction block, which may be an explanation for the relative rareness of epicardial functional conduction blocks in rToF patients (1 out of 4 ).

Another potential cause of lines of functional block is electrical remodelling and one protein that may be involved in this process is connexin 43 (Cx43). Its expression can decrease, and it may be found in a more lateral location on cardiomyocytes. Cx43 abnormalities have been shown in an animal model of $\mathrm{rToF}^{14,15}$, and also in RV samples of $\mathrm{rToF}$ patients ${ }^{16}$.

Unfortunately, no imaging data regarding cardiac fibrosis was available in any of the patients, but we can speculate about the reasons for finding structural blocks in the RVOT, particularly at the junction of the RVFW and the septum. Firstly, fibrotic tissue has been frequently observed at the RVFW-septum junction in patients with ToF $^{17}$. Secondly, even in normal hearts tissue architecture is less organized at the ventricular junctions than elsewhere in the ventricular walls ${ }^{18}$. It should be noted, however, that the patients that were included in the present study all underwent electrical mapping to search for foci of arrhythmia, meaning that they represent the patients with the most severely affected tissue properties in their respective categories.

\section{Activation dispersion in rToF during ventricular pacing}

In this study we used ventricular pacing to specifically test conduction properties in the working myocardium. Doing so, it was observed that epicardial activation dispersion in both $\mathrm{RV}$ and LV of rToF patients was quite comparable to that in non-rToF patients. Therefore, these data indicate that even if there are demonstrable tissue conduction abnormalities (structural and/or functional block), overall conduction is hardly affected.

During RV pacing, endocardial RV activation dispersion did not increase in the RVOT or RVFW. This further supports the notion that cardiac tissue properties of the majority of the RV areas are probably similar, since e.g. extensive fibrosis, electrical uncoupling or patch are expected to create zones of conduction delay or blocks $s^{6,7,12}$. The fact that the endocardially measured RVFW activation dispersion and its epicardial equivalent are in the same range and show comparable behaviour further underlines the relative tissue homogeneity in rToF hearts.

\section{Extrapolation to resynchronization of rToF patients}

Since RBBB in rToF patients is so prevalent, RV resynchronization through CRT is a tantalizing possibility. This may be especially true in hearts with RV hypertrophy (which may remain 
present in patients with rToF, due to residual pulmonary stenosis), since studies in dogs have shown that the negative hemodynamic effects of RBBB are more pronounced if the RV is hypertrophied ${ }^{19}$. Moreover, dyssynchrony itself leads to extensive ventricular remodelling, potentially further worsening ventricular function and increasing the risk of arrhythmia ${ }^{20}$.

Analogous to the abnormal contraction pattern observed in the LV of patients with LBBB, patients with rToF and RBBB show dyssynchronous contraction in the RV ${ }^{21}$. Indeed, several small studies have shown that CRT can improve synchrony of activation and cardiac function in patients with rToF, either through RV pacing aimed at fusion with the intrinsic activation wavefront or through biventricular pacing ${ }^{22-24}$. Even more interesting is the finding in a combined patient and computer simulation study that correction of the dyssynchrony in rToF patients may be of larger benefit to $\mathrm{RV}$ function and exercise tolerance than surgical correction of pulmonary valve regurgitation ${ }^{25}$.

Although we did not investigate CRT, our findings underline the potential to resynchronize the ventricles because our data primarily indicate conduction blocks proximal in the rapid conduction system. Moreover, because functional lines of block are also commonly found in patients with LBBB $^{26}$ (in whom CRT has become an established treatment ${ }^{27}$ ) we do not believe that their presence precludes successful CRT in rToF patients. In addition, activation dispersion in the RV and LV during ventricular pacing was comparable in rToF and in LBBB patients.

\section{Limitations}

This study has several limitations. Firstly, the number of patients that was studied was small and no healthy controls were included. Both are not surprising given the invasive nature of the mapping procedure. Only one pacing site was studied, and this site was not standardized for all patients. In comparison to studies focusing on the presence of isthmuses leading to ventricular arrhythmias ${ }^{28}$, the density of electrodes in the most basal part of the RVOT of the mapping arrays used in the present study may have been somewhat lower.

\section{Conclusions}

In rToF patients, structural lines of block are rare. Dispersion of activation in both endo- and epicardium of the RVFW is virtually unchanged between SR and RV pacing. Furthermore, activation dispersion in both RV and LV during pacing is comparable with that in non-BBB and LBBB patients. These findings indicate that the delayed activation in the RV of rToF patients is predominantly due to blocks in the Purkinje system, while conduction properties in the RV are relatively normal. 


\section{References}

1. Apitz C, Webb GD, Redington AN. Tetralogy of Fallot. Lancet 2009;374:1462-71.

2. D’Andrea A, Caso P, Sarubbi B, D'Alto M, Giovanna Russo M, Scherillo M, et al. Right ventricular myocardial activation delay in adult patients with right bundle branch block late after repair of Tetralogy of Fallot. Eur J Echocardiogr 2004;5:123-31.

3. Horowitz LN, Alexander JA, Edmunds LH. Postoperative right bundle branch block: identification of three levels of block. Circulation 1980;62:319-28.

4. Horowitz LN, Simson MB, Spear JF, Josephson ME, Moore EN, Alexander JA, et al. The mechanism of apparent right bundle branch block after transatrial repair of Tetralogy of Fallot. Circulation 1979;59:1241-52.

5. Steeg CN, Krongrad E, Davachi F, Bowman FO, Malm JR, Gersony WM. Postoperative left anterior hemiblock and right bundle branch block following repair of Tetralogy of Fallot. Clinical and etiologic considerations. Circulation 1975;51:1026-9.

6. Jalal Z, Sacher F, Fournier E, Cochet H, Derval N, Haissaguerre M, et al. Right Ventricular Electrical Activation in Patients With Repaired Tetralogy of Fallots. Circ Arrhythmia Electrophysiol 2019;12:e007141.

7. Kawara T, Derksen R, Groot JR de, Coronel R, Tasseron S, Linnenbank AC, et al. Activation delay after premature stimulation in chronically diseased human myocardium relates to the architecture of interstitial fibrosis. Circulation 2001;104:3069-75.

8. Nanthakumar K, Massé S, Poku K, Silversides CK, Chauhan VS, Mariani JA, et al. Intraoperative High-Density Global Mapping in Adult-Repaired Tetralogy of Fallot. Altered Left Ventricular and Right Ventricular Activation and Implications for Resynchronization Strategies. J Am Coll Cardiol 2010;55:2409-11.

9. Massé S, Downar E, Chauhan V, Sevaptsidis E, Nanthakumar K. Ventricular fibrillation in myopathic human hearts: mechanistic insights from in vivo global endocardial and epicardial mapping. AJP Hear Circ Physiol 2007;292:H2589-97.

10. Jia P, Ramanathan C, Ghanem RN, Ryu K, Varma N, Rudy Y. Electrocardiographic imaging of cardiac resynchronization therapy in heart failure: Observation of variable electrophysiologic responses. Hear Rhythm 2006;3:296-310.

11. Ruijter FTH De, Weenink I, Hitchcock FJ, Meijboom EJ, Bennink GBWE. Right ventricular dysfunction and pulmonary valve replacement after correction of Tetralogy of Fallot. Ann Thorac Surg 2002;73:1794-800.

12. Dhein S, Seidel T, Salameh A, Jozwiak J, Hagen A, Kostelka M, et al. Remodeling of cardiac passive electrical properties and susceptibility to ventricular and atrial arrhythmias. Front Physiol 2014;5:424.

13. Cochet $\mathrm{H}$, Iriart $\mathrm{X}$, Allain-Nicolaï $\mathrm{A}$, Camaioni $\mathrm{C}$, Sridi $\mathrm{S}$, Nivet $\mathrm{H}$, et al. Focal scar and diffuse myocardial fibrosis are independent imaging markers in repaired tetralogy of Fallot. Eur Heart $J$ Cardiovasc Imaging 2019;20:990-1003.

14. Benoist D, Dubes V, Roubertie F, Gilbert SH, Charron S, Constantin M, et al. Proarrhythmic remodelling of the right ventricle in a porcine model of repaired tetralogy of Fallot. Heart 2017;103:347-54. 
15. Dubes V, Benoist D, Roubertie F, Gilbert SH, Constantin M, Charron S, et al. Arrhythmogenic Remodeling of the Left Ventricle in a Porcine Model of Repaired Tetralogy of Fallot. Circ Arrhythm Electrophysiol 2018;11:e006059.

16. Kołcz J, Drukała J, Bzowska M, Rajwa B, Korohoda W, Malec E. The expression of connexin 43 in children with Tetralogy of Fallot. Cell Mol Biol Lett 2005;10:287-303.

17. Babu-Narayan S V., Kilner PJ, Li W, Moon JC, Goktekin O, Davlouros PA, et al. Ventricular fibrosis suggested by cardiovascular magnetic resonance in adults with repaired Tetralogy of Fallot and its relationship to adverse markers of clinical outcome. Circulation 2006;113:405-13.

18. Kuribayashi T, Roberts WC. Myocardial disarray at junction of ventricular septum and left and right ventricular free walls in hypertrophic cardiomyopathy. Am J Cardiol 1992;70:1333-40.

19. Yasui H, Yoshitoshi M, Komori M, Tominaga R, Kawachi Y, Ueno Y, et al. Cardiodynamic effects of experimental right bundle branch block in canine hearts with normal and hypertrophied right ventricles. Am Heart J 1985;109:69-77.

20. Nguyên UC, Verzaal NJ, Nieuwenhoven FA van, Vernooy K, Prinzen FW. Pathobiology of cardiac dyssynchrony and resynchronization therapy. Europace 2018;20:1898-909.

21. Hui W, Slorach C, Dragulescu A, Mertens L, Bijnens B, Friedberg MK. Mechanisms of right ventricular electromechanical dyssynchrony and mechanical ineffciency in children after repair of tetralogy of Fallot. Circ Cardiovasc Imaging 2014;7:610-8.

22. Thambo J-B, Guillebon M De, Xhaet O, Santos P Dos, Roubertie F, Labrousse L, et al. Biventricular pacing in patients with Tetralogy of Fallot: non-invasive epicardial mapping and clinical impact. Int $J$ Cardiol 2013;163:170-4.

23. Vojtovic P, Kucera F, Kubuš P, Gebauer R, Matejka T, Tláskal T, et al. Acute right ventricular resynchronization improves haemodynamics in children after surgical repair of tetralogy of Fallot. Europace 2018;20:323-8.

24. Plymen CM, Finlay M, Tsang V, O'leary J, Picaut N, Cullen S, et al. Haemodynamic consequences of targeted single- and dual-site right ventricular pacing in adults with congenital heart disease undergoing surgical pulmonary valve replacement. Europace 2015;17:274-80.

25. Lumens J, Fan C-PS, Walmsley J, Yim D, Manlhiot C, Dragulescu A, et al. Relative Impact of Right Ventricular Electromechanical Dyssynchrony Versus Pulmonary Regurgitation on Right Ventricular Dysfunction and Exercise Intolerance in Patients After Repair of Tetralogy of Fallot. J Am Heart Assoc 2019;8:e010903.

26. Auricchio A, Fantoni C, Regoli F, Carbucicchio C, Goette A, Geller C, et al. Characterization of Left Ventricular Activation in Patients with Heart Failure and Left Bundle-Branch Block. Circulation 2004;109:1133-9.

27. Brignole M, Auricchio A, Baron-Esquivias G, Bordachar P, Boriani G, Breithardt O-A, et al. 2013 ESC guidelines on cardiac pacing and cardiac resynchronization therapy: the task force on cardiac pacing and resynchronization therapy of the European Society of Cardiology (ESC). Developed in collaboration with the European Heart Rhythm Association. Europace 2013;34:2281-2329.

28. Kapel GFL, Sacher F, Dekkers OM, Watanabe M, Blom NA, Thambo J-B, et al. Arrhythmogenic anatomical isthmuses identified by electroanatomical mapping are the substrate for ventricular tachycardia in repaired Tetralogy of Fallot. Eur Heart J 2017;38:268-76. 


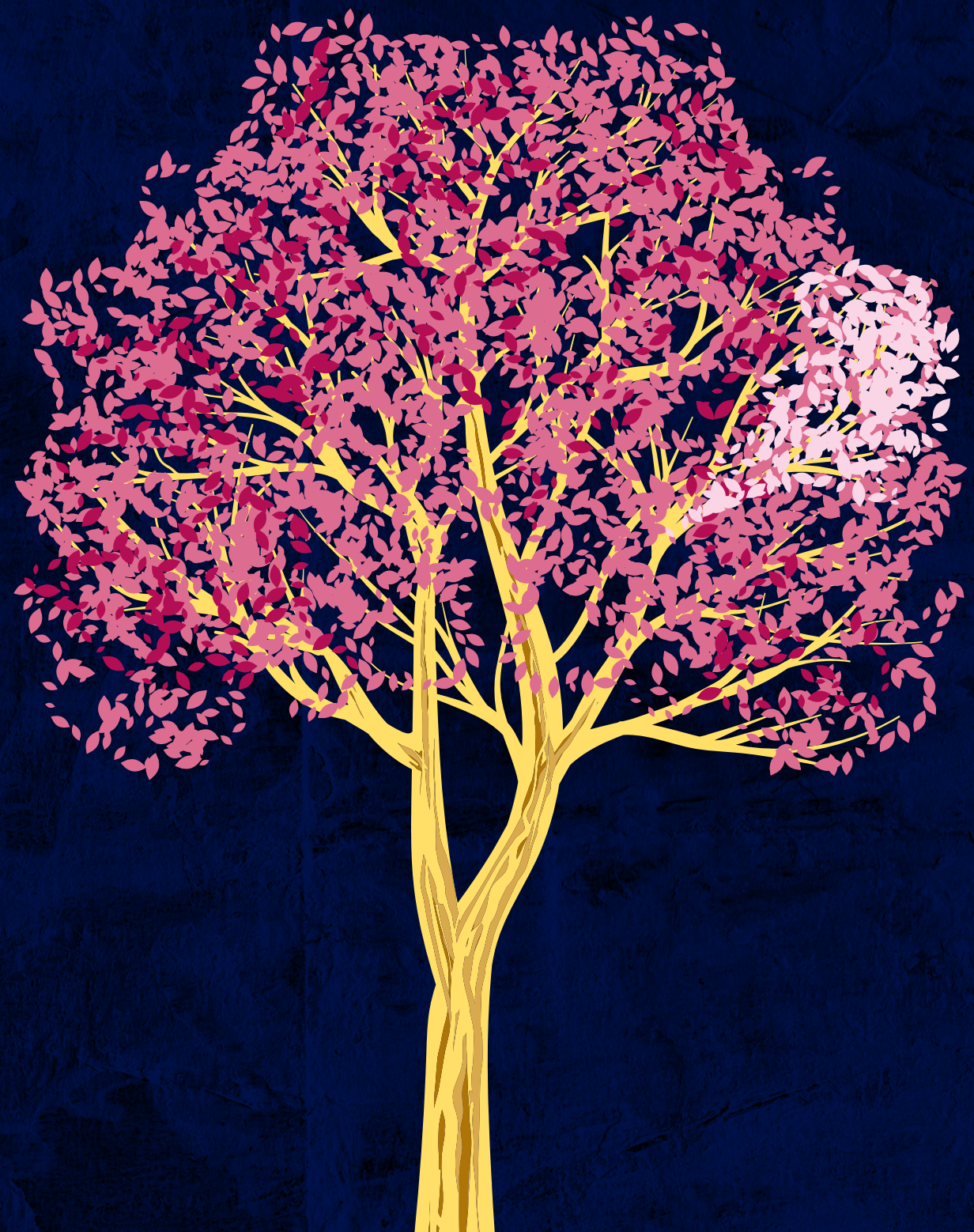




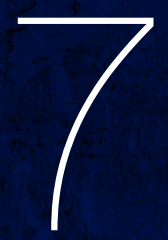

General discussion 

In the context of left bundle branch block (LBBB) resynchronization of depolarization through cardiac resynchronization therapy (CRT) and its effect on pump function and long-term outcome have been studied extensively. This thesis aimed to shed some light on aspects of CRT that have not yet been studied in detail.

In chapters $\mathbf{3}$ and $\mathbf{4}$ we investigated remodelling of repolarization following CRT, using changes in T wave area as a marker, both during CRT and after short interruption of CRT. We showed that repolarization remodelling occurs rapidly (within 2 weeks of starting CRT). Simulations in a patient-specific computational model demonstrated that this repolarization remodelling leads to lower dispersion of repolarization. Additionally, more repolarization remodelling was linked to a better echocardiographic response to CRT.

In chapter 5 we explored the effects of CRT in an animal model of LBBB and structural mitral regurgitation (MR). While CRT most likely does not influence this kind of MR, we found evidence that CRT still increases left ventricular (LV) pump function.

Finally, in chapter 6, we investigated the tissue conduction properties of patients with repaired Tetralogy of Fallot (rToF) and compared these patients to a small group of LBBB and nonbundle branch block (non-BBB) patients. We found that structural conduction blocks were rare in patients with rToF and that tissue conduction properties of the right ventricular (RV) free wall were comparable between rToF and other patients.

\section{Repolarization remodelling following CRT}

Our finding that the change in depolarization pattern by CRT influences repolarization is in line with previous studies showing that $\mathrm{T}$ area and/or amplitude increase when the CRT device is switched off after a period of pacing ${ }^{1-3}$. These previous studies demonstrated that these changes already occurred well within a week after initiation of $\mathrm{CRT} \mathrm{T}^{1-3}$.

New aspects in our studies, however, are the longer time period that was investigated (14-90 days vs. 6 months in our studies), the more frequent vectorcardiographic (VCG) recordings and the larger number of patients involved (11-23 biventricularly paced patients vs. 25 (chapter 3) and 76 (chapter 4)). Moreover, to the best of our knowledge, we are the first to investigate a possible link between $T$ area changes and increase in left ventricular ejection fraction (LVEF). Furthermore, we elaborated on the use of T wave area during brief interruption of CRT (CRTOFF) as marker of dispersion of repolarization. To this purpose we also made use of a computer model that linked the increase in T area during CRT-OFF to a change in repolarization sequence, indicating a reduction in dispersion of repolarization during longer-lasting CRT. 
Upon starting CRT, T area is acutely reduced. This reduction seems to be explained by the fact that during CRT the two depolarizing wavefronts from the ventricular pacing electrodes give rise to two repolarization wavefronts that merge and cancel each other, thus reducing $T$ area. Our findings extend the findings by Wecke et al., who also found a reduction in $\mathrm{T}$ area during $\mathrm{CRT}^{1}$.

Adaptation in repolarization due to longer-lasting altered activation sequence has been evidenced by $T$ wave changes following onset of RV pacing or $\mathrm{LBBB}^{4-6}$. Under those conditions the transition from synchronous to dyssynchronous activation led to an immediate large increase in T wave amplitude and area, followed by a moderate reduction in these parameters during longer-lasting dyssynchrony ${ }^{4-6}$. Adaptations in repolarization have also been indicated by an abnormal $T$ wave occurring after brief interruption of RV pacing: return of the narrow QRS complex was accompanied by a T wave pointing in the direction of the QRS complex during pacing ("T wave memory") ) $^{5,7}$. Studies in rabbit hearts demonstrated that such changes in repolarization may occur due to prolongation of the action potential duration (APD) in earlyactivated regions and APD shortening in later-activated regions ${ }^{8}$.

Our research differs from these aforementioned studies, since during CRT the activation changes from dyssynchronous to more synchronous, so opposite to the work investigating the effect of chronic RV pacing or LBBB. Like the aforementioned publications, we did observe an abnormal T wave upon interrupting CRT in our analysis. However, we did not see a chronic reduction in T area over time during continuous CRT (CRT-ON). This observation may be explained by dominance of the CRT-induced bifocal spread of activation over the repolarization sequence, which may conceal regional repolarization changes.

Nonetheless, a small study (11 patients) by Padeletti et al. did find a decrease in $T$ wave amplitude during persistent $\mathrm{CRT}^{3}$. In that study, however, T amplitude increased immediately upon start of CRT, compared to the baseline situation. Moreover, in that study, the T amplitude also remained higher than baseline after 60 days of CRT. Possible explanations for these different findings are differences in composition of the patient cohorts and differences in measurement of the VCG. In our study, for example, the majority of patients was in NYHA class I-III whereas in the study by Padeletti et al., all patients were in NYHA class III-IV. While we constructed the VCG from the 12-lead electrocardiogram (ECG)(validated by Engels ${ }^{9}$ et al.), Padeletti et al. used direct measurement from the Marquette system. Finally, the first measurement during CRT was on the day after implantation in our studies but immediately upon device implantation by Padeletti et al.

Interestingly, brief interruption of CRT unveiled changes in T area over time that were not (or hardly) observed during CRT-ON. During CRT-OFF $\mathrm{T}$ area was also the parameter that 
showed changes more pronouncedly than several commonly used markers of repolarization, such as QT interval, T peak-to-end interval $\left(T_{p-e}\right)$ and precordial QT dispersion. One of the possible implications of the present study may be that briefly switching off the CRT device and measuring $T$ area may be used to investigate repolarization remodelling in greater detail.

An advantage of the use of $\mathrm{T}$ area to study this phenomenon is that it is easy to objectively and reliably quantify. After all, $T$ area is largely determined by the amplitude of the major part of the $T$ wave. In contrast, determining the end of the $T$ wave for repolarization markers like QT interval and $T_{p-e}$ can be subjective ${ }^{10}$, although recent development of an algorithm may make these measurements more objective ${ }^{11}$.

\section{Relation between repolarization remodelling and arrhythmias?}

There is conflicting data on the effect of CRT on the occurrence of arrhythmias. A shortterm study in patients indicated a potential pro-arrhythmic effect of CRT evidenced by increased dispersion of repolarization ${ }^{12}$. When epicardial pacing was applied to animal tissues an increase in dispersion of repolarization was also observed, and arrhythmias could be induced after administering potassium channel blockers ${ }^{13}$. However, a reduction in arrhythmia incidence following CRT in responders was found in several other studies, including clinical trials, that also demonstrated an increased arrhythmia incidence in non-responders ${ }^{14,15}$. Such findings suggest that the reduction in arrhythmias is related to the reverse remodelling in CRT responders.

In general, a larger dispersion of repolarization is considered to be a risk factor for the occurrence of arrhythmias. Despite all the changes we observed in repolarization parameters, arrhythmia incidence was low and did not change over time in the patient cohorts we studied. The fact that there were very few ventricular arrhythmias is a likely reason for this finding. This observation becomes even more interesting when considering that biventricular pacing is not present during all heartbeats, because even during well-installed CRT the percentage biventricular pacing is usually less than $100 \%$, leaving $~ 1-5 \%$ of the beats with the same increased dispersion of repolarization as we observed during CRT-OFF ${ }^{16}$.

On the one hand, finding a possible relation between repolarization and arrhythmias would require a considerably larger study. On the other hand, there are at least two other potential explanations for a lack of a clear relation between dispersion of repolarization and arrhythmias. First, T area and the other repolarization parameters are fairly global measures of repolarization dispersion, while local repolarization dispersion is more likely to be particularly important for arrhythmia risk ${ }^{17}$. Second, as shown by the patient-specific computational model, in CRT 
patients the repolarization "waves" are likely large and broad with small local repolarization gradients. This has also been shown by direct mapping in a similar patient cohort ${ }^{18}$. Under such conditions it is unlikely that re-entry occurs.

\section{Possible mechanisms of repolarization remodelling}

Mechano-electrical coupling has been proposed as an explanation for the repolarization remodelling observed during both $\mathrm{LBBB}$ and CRT. Sosunov et al. provided elegant evidence for this idea by showing that in rabbit hearts reducing or preventing pressure development in the ventricles precluded the development of $\mathrm{T}$ wave memory ${ }^{19}$. A more cellular mechanistic view was provided by Kuijpers et al. ${ }^{20}$ As the heart adapts to the new activation sequence, calcium currents may change locally to re-homogenize the amount of work each region has to perform ${ }^{20}$. According to this assumption, in early-activated areas calcium currents may increase to compensate for the reduction in work, which in turn increases local APD. The opposite may be true for areas that are activated late ${ }^{20}$. Over time this may lead to a lower dispersion of repolarization times ${ }^{20}$, which can cause a reduction in $T$ area.

Data in chapter 3 indicate that repolarization remodelling precedes changes in LVEF. This may seem to contradict a relation between repolarization and contractile remodelling as described above. However, both LVEF and strain are related to LV volumes and volume reduction after CRT takes several weeks ${ }^{21,22}$.

\section{Remodelling in impulse conduction?}

While the focus of our work was on changes in the T wave, we noticed some unexpected changes in the QRS complex during longer-lasting CRT. In chapter 3, we observed a modest, transient increase in QRS area during the first month of CRT. This increase seems to disappear after a few months, which may explain why we did not observe this phenomenon in chapter $\mathbf{4}$, where we only studied baseline and remodelling after 6 months.

However, in chapter 4, we found that QRS area did not change between baseline and 6-month follow-up in patients with a large increase in T area during CRT-OFF, whereas QRS area decreased in the patients with a small change in T area.

In an attempt to link these two observations, we stratified 21 patients from chapter $\mathbf{3}$ by the median T area change we observed in chapter $\mathbf{4}$ (smaller than $41 \mathrm{mV} \cdot \mathrm{ms}$ : LOW, larger than $41 \mathrm{mV} \cdot \mathrm{ms}$ : HIGH). Although differences were not statistically significant (likely due to the small group sizes: LOW $=5$ patients, $\mathrm{HIGH}=16$ patients), QRS area tended to be smaller in the LOW subgroup than in the HIGH subgroup at baseline and during all CRT-OFF measurements 
(Figure 1). In both subgroups QRS area showed a similar trend of a temporary increase during the first month of CRT. However, the reduction in QRS area we observed after 6 months in the LOW subgroup in chapter $\mathbf{4}$ is not visible in the LOW subgroup shown in Figure 1.

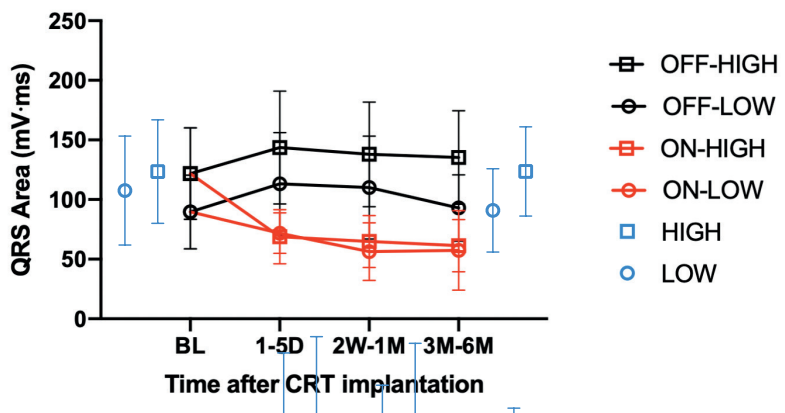

FIGURE 1. QRS area over time in 21 patients from chapter 3, for whom baseline and 3- or 6-month ECGs with stable morphologies were available. Patients were stratified according to $T$ area change between baseline and late followup during CRT-OFF (LOW = smaller than $41 \mathrm{mV} \cdot \mathrm{ms}, 5$ patients; $\mathrm{HIGH}=$ larger than $41 \mathrm{mV} \cdot \mathrm{ms}, 16$ patients). Red and black lines represent $\mathrm{ON}$ and OFF measurements for the indicated subgroup. Blue symbols represent values from baseline and at 6 months CRT-OFF from chapter 4, Figure $2 \mathrm{~B}$. $\mathrm{BL}=$ baseline, $1-5 \mathrm{D}=1-5$ days, $2 \mathrm{~W}-1 \mathrm{M}=2$ weeks-1 month, 3M-6M = 3-6 months, $\mathrm{CRT}=$ cardiac resynchronization therapy, ECG = electrocardiogram, $\mathrm{ON}=$ during $\mathrm{CRT}$, OFF = during brief interruption of CRT.

A large QRS area suggests unopposed depolarization ${ }^{23}$. Animal studies indicated that dyssynchrony can bring about abnormal connexin 43 (Cx43) expression and localization ${ }^{24,25}$. Stretching cultured cardiomyocytes causes Cx43 relocalization ${ }^{26}$, and major local mechanical changes can be induced by dyssynchrony. Taken together, these findings support the idea that myocardial impulse conduction may be affected by dyssynchrony-induced mechanical derangements. Moreover, cellular uncoupling can lower QRS amplitudes ${ }^{27}$. Therefore, it may be speculated that a more polarized $\mathrm{C} \times 43$ localization and/or an increase in $\mathrm{Cx} 43$ expression may be responsible for the increase in QRS area after CRT, through a more uniform (but not overall faster) depolarization.

\section{Future perspectives}

Given the relatively small number of studies on repolarization in CRT patients, the present studies may give cause for further research on this topic. While we have shown a positive correlation between $T$ wave area change and echocardiographic response during mediumterm follow-up, it is unclear whether a larger $T$ area change is also linked to a larger clinical benefit. Studies with more patients and longer follow-up are needed to additionally investigate the connection between repolarization remodelling and clinical CRT response, including 
arrhythmia risk. Better investigation of the relation between $T$ area changes and arrhythmias will require a larger study than the one performed by us, in particular in order to examine subgroups of patients that are known to be more vulnerable to arrhythmias, such as patients with a history of ischemic cardiomyopathy.

Additionally, to the best of our knowledge, it is not known whether more synchronous repolarization following CRT may also lead to improved ventricular relaxation, which in turn could beneficially affect diastolic function. In this regard it is interesting to note that the first studies on acute haemodynamic response to CRT found a consistent improvement in systolic function (maximum rate of LV pressure rise, $\mathrm{LVdP} / \mathrm{dt}_{\text {max }}$ ), but changes in diastolic function, as quantified by maximum rate of $\mathrm{LV}$ pressure fall $\left(\mathrm{LVdP} / \mathrm{dt}_{\min }\right)$ and the time constant of $\mathrm{LV}$ pressure decay (tau) were non-significant or small and inconsistent ${ }^{28,29}$. In canine hearts with 8 weeks of LBBB it was found that LVdP/dt ${ }_{\text {min }}$ decreased from approximately -1600 to $-1870 \mathrm{mmHg} / \mathrm{s}$ immediately after start of CRT and further decreased significantly to about $-2020 \mathrm{mmHg} / \mathrm{s}^{30}$. In this study electrical contact mapping also showed an altered relation between activation time and APD, agreeing with the changes observed in the computer simulations in chapter $\mathbf{3}$. Therefore, it seems worthwhile to further investigate the relation between repolarization and relaxation changes in CRT patients.

Furthermore, a more detailed mechanistical study that can provide additional information about the role of mechano-electrical coupling would be of interest. Such a mechanistical study could, for example, make use of a new module of the CircAdapt computer model that provides, through the buffering of calcium by troponin, bidirectional coupling between the O'Hara-Rudy model of human ventricular electrophysiology and the MechChem model of sarcomere mechanics ${ }^{31}$.

If the mechanisms behind these changes are understood, a possible practical implication of our findings may be that brief interruption of CRT can be used as a diagnostic test to observe changes in $\mathrm{T}$ wave area, which may yield insight into molecular changes in the myocardium of the patient. Because these changes occur relatively fast (within 2 weeks), one does not need to wait for 3-6 months, which is the period used to evaluate CRT response echocardiographically ${ }^{32}$, in order to consider potential adjustments to the therapy.

\section{CRT during mitral regurgitation}

MR often occurs in patients with heart failure, and its severity is linked to QRS duration ${ }^{33,34}$. CRT may be able to reduce dyssynchrony-related (functional) MR through several mechanisms ${ }^{33}$. Firstly, the closing force exerted on the mitral valve leaflets (by ventricular contraction) may 
increase due to the more efficient cardiac contraction ${ }^{35}$. Secondly, resynchronization of the papillary muscles may result in improved positioning of the valve leaflets, optimizing valve closure ${ }^{36}$. Thirdly, a reduction in the size of the mitral orifice may reduce $\mathrm{MR}^{37}$. Finally, diastolic MR may be reduced by an improvement in the relative timing of atrial and ventricular contraction $^{38}$.

However, it is not yet clear whether CRT may benefit patients with structural MR, which is not related to dyssynchronous contraction ${ }^{39}$. Our study in the MR+LBBB dog model (chapter 5) shows that CRT could still be beneficial in patients with LBBB and structural MR. The most likely mechanism for this improvement is resynchronization of ventricular contraction, i.e. counteracting the negative effects of LBBB.

It is important to remember, however, that the increase in $\mathrm{LVdP} / \mathrm{dt}_{\max }$ we observed may not directly indicate more forward flow. Most importantly, CRT led to either an increase in LVdP/dt ${ }_{\max }$ or a decrease in LV end-diastolic pressure and in most cases both. This indicates alleviation of either forward or backward failure or both, but the exact mechanism and its effects on cardiac output remain to be investigated.

Moreover, it is not yet known whether acute positive effects of CRT on cardiac dyssynchrony in patients with LBBB and structural MR would also lead to long-term reverse cardiac remodelling and improved clinical outcomes. Earlier studies describing effects of CRT in the context of MR often excluded patients with structural $\mathrm{MR}^{36,40-42}$.

If a reduction in MR does indeed lead to better CRT outcomes, one could propose that residual MR after CRT should be treated using mitral valve repair. The COAPT trial supports this idea, since it demonstrated that patients who had previously received CRT could still derive benefit from a MitraClip device over optimal medical therapy alone in relation to the combined end point of heart failure hospitalizations and all-cause mortality ${ }^{43}$. However, another study showed that implantation of a MitraClip device did not seem to have a large beneficial effect in CRT non-responders who also suffer from significant $\mathrm{MR}^{44}$. An important difference between these two studies is that the latter did not include a control group but predicted survival scores using patient characteristics, while the former directly compared MitraClip plus optimal medical therapy with optimal medical therapy alone. Moreover, the group sizes were very different (614 vs. 42 patients).

The finding that CRT may even be effective in the volume-overloaded dyssynchronous ventricle could also be good news for patients with rToF, who often have right bundle branch block (RBBB) and pulmonary insufficiency following repair ${ }^{45}$. A study in dog hearts suggests that RBBB may have more severe negative effects on cardiac function in the presence of RV hypertrophy, 
created by juvenile pulmonary banding ${ }^{46}$. Conversely, counteracting RBBB through CRT may be extra beneficial for cardiac function in this scenario, which can occur in rToF patients due to remaining pulmonary stenosis and concomitant RV pressure overload. However, the net effect of the combination of pressure and volume overload during dyssynchrony and CRT remains to be investigated.

\section{Future perspectives}

To date, patients with structural MR are often excluded from studies focusing on CRT in the context of this disease ${ }^{36,40,42}$. It would be interesting to see if our findings in the animal model are replicated in those patients, and, more importantly, whether any short-term improvement also relates to long-term benefit. In practice, these findings may be of particular interest to patients of advanced age, who may be at higher risk of complications following mitral repair.

\section{Conduction properties in patients with rToF}

RBBB is common in ToF patients after repair surgery and can occur either proximally, due to the closing of the ventricular septal defect, or more distally, due to the right ventricular outflow tract obstruction resection or ventriculotomy ${ }^{47,48}$, or fibrosis due to RV overload. Theoretically, this conduction abnormality is an attractive target for CRT. However, the presence of myocardial scar tissue (e.g. due to the repair surgery or to RV overload) may limit the amount of resynchronization and the related CRT benefit ${ }^{49}$. We found that structural conduction blocks were rare in a small group of rToF patients and that the conduction properties of the RV free wall were fairly normal. Of note, these measurements were performed during open-heart surgery for medical reasons, more than two decades after the initial repair operation. Therefore, the patients we studied represent a group of heavily affected rToF patients. The absence of conduction blocks would allow biventricular pacing to resynchronize cardiac contraction, much like in patients with LBBB and RBBB of other cause.

However, the benefit of CRT in RBBB is disputed ${ }^{50}$. One of the reasons for this may be the fact that, in most studies, CRT benefit is quantified by assessing LV function or volume ${ }^{50}$. However, in rToF patients, RV function may be the proverbial bottleneck hampering cardiac function, suggesting that CRT benefits may be assessed more clearly when focusing on this ventricle ${ }^{48,50}$.

Another reason for the apparent lack of benefit of CRT in RBBB may be related to lead location ${ }^{50}$. While placing leads on the LV free wall and in the RV apex may be beneficial for LBBB, this may not be the optimum lead configuration for patients with RBBB ${ }^{50}$. Computer simulations demonstrated that traditional CRT was hardly beneficial in patients with isolated RBBB if no 
LBBB was present ${ }^{50}$. A study in patients with congenital heart disease and RBBB found that pacing from a site of late activation in the RV yielded superior haemodynamic benefit over RV apical pacing, underlining the importance of exploring alternative lead locations ${ }^{51}$. Pacing the $\mathrm{RV}$ from a site of late activation was also found to be beneficial in other studies ${ }^{52,53}$.

Two studies found, however, that RV only pacing in rToF did not lead to cardiac resynchronization ${ }^{54,55}$. However, in one of these studies, the lead was not necessarily placed in a site of late activation ${ }^{54}$, while in the other study, a relatively short atrioventricular (AV) delay was used (70\% of the intrinsic PR interval) ${ }^{55}$. The study by Plymen et al. used a longer AV delay (close to $90 \%$ of the intrinsic PR interval), potentially yielding more fusion with the intrinsic activation wavefront and thus more effective resynchronization ${ }^{51}$.

Nonetheless, it is not yet clear whether RV only pacing would be sufficient in rToF. LV activation can also be abnormal in patients with $r$ ToF, either due to scarring ${ }^{56}$ or left anterior hemiblock ${ }^{57}$. In our study, we did find that the four rToF patients we studied with the epicardial sock array had a region of late activation anteriorly in the LV, although it was small in two patients. However, the average LV activation time in the rToF patients was not very different from the non-bundle branch block (non-BBB) patients (Chapter 6, Figure 5B), which would suggest that LV activation in the rToF patients was fairly normal. However, it is important to remember that the non-BBB patients in this study also underwent electrical mapping to search for foci of arrhythmia, indicating that they were not healthy controls.

Besides RV pacing, also biventricular pacing may lead to electrical resynchronization in rToF patients ${ }^{54,58}$, but it is not clear whether this type of resynchronization is superior to RV only pacing from a site of late activation.

Regardless of the optimum pacing site and setting, RBBB may negatively affect cardiac function, and dyssynchrony may be an even more important limiting factor than pulmonary insufficiency for exercise capacity in patients with rToF ${ }^{59}$. The aetiology of RBBB in rToF (surgical) is different from RBBB in the general population, which is linked to degenerative cardiac disease $^{60}$ and therefore potentially to tissue properties that may preclude CRT response. From that perspective, rToF patients may benefit more from CRT than other RBBB patients.

\section{Future perspectives}

If CRT were to become a treatment for RBBB in rToF, it would be important to determine the optimum time of implantation. Implantation immediately following surgery would have the advantage of prompt resynchronization, which may be important for improvement in exercise capacity in patients with rToF$^{59}$. However, pacemaker implantation in children may carry a 
higher risk of infection or lead malfunction than in adults ${ }^{61}$. Our study suggests that, even in adult patients with rToF and severe arrhythmias, cardiac conduction properties do not preclude successful resynchronization.

\section{Conclusions}

The goal of this thesis was to provide some insight into relatively unexplored aspects of CRT. Our results indicate that repolarization remodelling after starting CRT occurs rapidly and may lead to lower dispersion of repolarization. Additionally, more repolarization remodelling may be linked to a better echocardiographic response to CRT. In a dog model, we also found evidence that CRT still increases LV pump function in the presence of structural MR. Finally, our studies show that structural conduction blocks are rare in patients with $\mathrm{rToF}$ and that tissue properties of the right ventricular free wall are comparable between rToF patients and LBBB or non-BBB patients. 


\section{References}

1. Wecke L, Deursen CJM Van, Bergfeldt L, Prinzen FW. Repolarization changes in patients with heart failure receiving cardiac resynchronization therapy - Signs of cardiac memory. J Electrocardiol 2011;44:590-8.

2. Perrotta L, Ricciardi G, Pieragnoli P, Nesti M, Pontecorboli G, Fantini F, et al. Cardiac memory in cardiac resynchronization therapy: A vectorcardiographic comparison of biventricular and left ventricular pacing. J Electrocardiol 2015;48:571-7.

3. Padeletti L, Fantappiè C, Perrotta L, Ricciardi G, Pieragnoli P, Chiostri M, et al. Cardiac memory in humans: Vectocardiographic quantification in cardiac resynchronization therapy. Clin Res Cardiol 2011;100:51-6.

4. Shvilkin A, Bojovic B, Vajdic B, Gussak I, Ho KK, Zimetbaum P, et al. Vectorcardiographic and electrocardiographic criteria to distinguish new and old left bundle branch block. Hear Rhythm 2010;7:1085-92.

5. Shvilkin A, Bojovic B, Vajdic B, Gussak I, Zimetbaum P, Josephson ME. Vectorcardiographic determinants of cardiac memory during normal ventricular activation and continuous ventricular pacing. Hear Rhythm 2009;6:943-8.

6. Engels EB, Poels TT, Houthuizen P, Jaegere PPT de, Maessen JG, Vernooy K, et al. Electrical remodelling in patients with iatrogenic left bundle branch block. Europace 2016;18:iv44-52.

7. Rosen MR, Bergfeldt L. Cardiac memory: The slippery slope twixt normalcy and pathology. Trends Cardiovasc Med 2015;25:687-96.

8. Costard-Jäckle A, Goetsch B, Antz M, Franz MR. Slow and long-lasting modulation of myocardial repolarization produced by ectopic activation in isolated rabbit hearts. Evidence for cardiac 'memory'. Circulation 1989;80:1412-20.

9. Engels EB, Alshehri S, Deursen CJM Van, Wecke L, Bergfeldt L, Vernooy K, et al. The synthesized vectorcardiogram resembles the measured vectorcardiogram in patients with dyssynchronous heart failure. J Electrocardiol 2015;48:586-92.

10. Viskin S, Rosovski U, Sands AJ, Chen E, Kistler PM, Kalman JM, et al. Inaccurate electrocardiographic interpretation of long QT: The majority of physicians cannot recognize a long QT when they see one. Hear Rhythm 2005;2:569-74.

11. Hermans BJM, Vink AS, Bennis FC, Filippini LH, Meijborg VMF, Wilde AAM, et al. The development and validation of an easy to use automatic QT-interval algorithm. PLoS One 2017;12:e0184352.

12. Medina-Ravell VA, Lankipalli RS, Yan G-X, Antzelevitch C, Medina-Malpica NA, Medina-Malpica $\mathrm{OA}$, et al. Effect of epicardial or biventricular pacing to prolong QT interval and increase transmural dispersion of repolarization: does resynchronization therapy pose a risk for patients predisposed to long QT or torsade de pointes? Circulation 2003;107:740-6.

13. Fish JM, Diego JM Di, Nesterenko V, Antzelevitch C. Epicardial Activation of Left Ventricular Wall Prolongs QT Interval and Transmural Dispersion of Repolarization: Implications for Biventricular Pacing. Circulation 2004;109:2136-42. 
14. Saini A, Kannabhiran M, Reddy P, Gopinathannair R, Olshansky B, Dominic P. Cardiac Resynchronization Therapy May Be Antiarrhythmic Particularly in Responders: A Systematic Review and Meta-Analysis. JACC Clin Electrophysiol 2016;2:307-16.

15. Deif B, Ballantyne B, Almehmadi F, Mikhail M, Mclntyre WF, Manlucu J, et al. Cardiac resynchronization is pro-arrhythmic in the absence of reverse ventricular remodelling: A systematic review and metaanalysis. Cardiovasc Res 2018;114:1435-44.

16. Hayes DL, Boehmer JP, Day JD, Gilliam FR, Heidenreich PA, Seth M, et al. Cardiac resynchronization therapy and the relationship of percent biventricular pacing to symptoms and survival. Hear Rhythm 2011;8:1469-75.

17. Arteyeva NV. Dispersion of ventricular repolarization: Temporal and spatial. World J Cardiol 2020;12:437-49.

18. Maffessanti F, Wanten J, Potse M, Regoli F, Caputo ML, Conte G, et al. The relation between local repolarization and T-wave morphology in heart failure patients. Int J Cardiol 2017;241:270-6.

19. Sosunov EA, Anyukhovsky EP, Rosen MR. Altered ventricular stretch contributes to initiation of cardiac memory. Hear Rhythm 2008;5:106-13.

20. Kuijpers NHL, Hermeling E, Lumens J, Eikelder HMM ten, Delhaas T, Prinzen FW. Mechano-electrical coupling as framework for understanding functional remodeling during LBBB and CRT. Am J Physiol - Hear Circ Physiol 2014;306:H1644-59.

21. Vernooy K, Cornelussen RNM, Verbeek XAAM, Vanagt WYR, Hunnik A Van, Kuiper M, et al. Cardiac resynchronization therapy cures dyssynchronopathy in canine left bundle-branch block hearts. Eur Heart J 2007;28:2148-55.

22. Yu CM, Chau E, Sanderson JE, Fan K, Tang MO, Fung WH, et al. Tissue Doppler echocardiographic evidence of reverse remodeling and improved synchronicity by simultaneously delaying regional contraction after biventricular pacing therapy in heart failure. Circulation 2002;105:438-45.

23. Deursen CJM Van, Vernooy K, Dudink E, Bergfeldt L, Crijns HJGM, Prinzen FW, et al. Vectorcardiographic QRS area as a novel predictor of response to cardiac resynchronization therapy. J Electrocardiol 2015;48:45-52.

24. Spragg DD, Akar FG, Helm RH, Tunin RS, Tomaselli GF, Kass DA. Abnormal conduction and repolarization in late-activated myocardium of dyssynchronously contracting hearts. Cardiovasc Res 2005;67:77-86.

25. Spragg DD, Leclercq C, Loghmani M, Faris OP, Tunin RS, DiSilvestre D, et al. Regional alterations in protein expression in the dyssynchronous failing heart. Circulation 2003;108:929-32.

26. Salameh A, Wustmann A, Karl S, Blanke K, Apel D, Rojas-Gomez D, et al. Cyclic mechanical stretch induces cardiomyocyte orientation and polarization of the gap junction protein connexin43. Circ Res 2010;106:1592-602.

27. Potse M, Krause D, Bacharova L, Krause R, Prinzen FW, Auricchio A. Similarities and differences between electrocardiogram signs of left bundle-branch block and left-ventricular uncoupling. Europace 2012;14:v33-9.

28. Kass DA, Chen CH, Curry C, Talbot M, Berger R, Fetics B, et al. Improved left ventricular mechanics from acute VDD pacing in patients with dilated cardiomyopathy and ventricular conduction delay. Circulation 1999;99:1567-73. 
29. Auricchio A, Stellbrink C, Block M, Sack S, Vogt J, Bakker P, et al. Effect of pacing chamber and atrioventricular delay on acute systolic function of paced patients with congestive heart failure. Circulation 1999;99:2993-3001.

30. Kerckhoffs RCP, Lumens J, Vernooy K, Omens JH, Mulligan LJ, Delhaas T, et al. Cardiac resynchronization: Insight from experimental and computational models. Prog Biophys $\mathrm{Mol}$ Biol 2008;97:543-61.

31. Lyon A, Dupuis LJ, Arts T, Crijns HJGM, Prinzen FW, Delhaas T, et al. Differentiating the effects of $\beta$-adrenergic stimulation and stretch on calcium and force dynamics using a novel electromechanical cardiomyocyte model. Am J Physiol - Hear Circ Physiol 2020;319:H519-30.

32. Daubert JC, Saxon L, Adamson PB, Auricchio A, Berger RD, Beshai JF, et al. 2012 EHRA/HRS expert consensus statement on cardiac resynchronization therapy in heart failure: Implant and follow-up recommendations and management. Europace 2012;14:1236-86.

33. Spartera M, Galderisi M, Mele D, Cameli M, D'Andrea A, Rossi A, et al. Role of cardiac dyssynchrony and resynchronization therapy in functional mitral regurgitation. Eur Heart J Cardiovasc Imaging 2016;17:471-80.

34. Erlebacher JA, Barbarash S. Intraventricular conduction delay and functional mitral regurgitation. Am $J$ Cardiol 2001;88:83-6.

35. Breithardt OA, Sinha AM, Schwammenthal E, Bidaoui N, Markus KU, Franke A, et al. Acute effects of cardiac resynchronization therapy on functional mitral regurgitation in advanced systolic heart failure. J Am Coll Cardiol 2003;41:765-70.

36. Bartko PE, Arfsten H, Heitzinger G, Pavo N, Strunk G, Gwechenberger M, et al. Papillary Muscle Dyssynchrony-Mediated Functional Mitral Regurgitation: Mechanistic Insights and Modulation by Cardiac Resynchronization. JACC Cardiovasc Imaging 2019;12:1728-37.

37. Porciani MC, Macioce R, Demarchi G, Chiostri M, Musilli N, Cappelli F, et al. Effects of cardiac resynchronization therapy on the mechanisms underlying functional mitral regurgitation in congestive heart failure. Eur J Echocardiogr 2006;7:31-9.

38. Salden FCWM, Kutyifa V, Stockburger M, Prinzen FW, Vernooy K. Atrioventricular dromotropathy: evidence for a distinctive entity in heart failure with prolonged PR interval? Europace 2018;20:106777.

39. Denney TS, Nagaraj HM, Lloyd SG, Aban I, Corros C, Seghatol-Eslami F, et al. Effect of Primary Mitral Regurgitation on Left Ventricular Synchrony. Am J Cardiol 2007;100:707-11.

40. Verhaert D, Popović ZB, De S, Puntawangkoon C, Wolski K, Wilkoff BL, et al. Impact of mitral regurgitation on reverse remodeling and outcome in patients undergoing cardiac resynchronization therapy. Circ Cardiovasc Imaging 2012;5:21-6.

41. John Sutton M St., Ghio S, Plappert T, Tavazzi L, Scelsi L, Daubert C, et al. Cardiac resynchronization induces major structural and functional reverse remodeling in patients with New York heart association class I/II heart failure. Circulation 2009;120:1858-65.

42. Bijl P van der, Khidir M, Ajmone Marsan N, Delgado V, Leon MB, Stone GW, et al. Effect of Functional Mitral Regurgitation on Outcome in Patients Receiving Cardiac Resynchronization Therapy for Heart Failure. Am J Cardiol 2019;123:75-83. 
43. Stone GW, Lindenfeld JA, Abraham WT, Kar S, Lim DS, Mishell JM, et al. Transcatheter mitral-valve repair in patients with heart failure. N Engl J Med 2018;379:2307-18.

44. Seifert M, Schau T, Schoepp M, Arya A, Neuss M, Butter C. MitraClip in CRT non-responders with severe mitral regurgitation. Int J Cardiol 2014;177:79-85.

45. Bove T, François K, Wolf D de. New insights into the surgical management of Tetralogy of Fallot: Physiological fundamentals and clinical relevance. Curr Pediatr Rev 2015;11:72-86.

46. Yasui H, Yoshitoshi M, Komori M, Tominaga R, Kawachi Y, Ueno Y, et al. Cardiodynamic effects of experimental right bundle branch block in canine hearts with normal and hypertrophied right ventricles. Am Heart J 1985;109:69-77.

47. Horowitz LN, Alexander JA, Edmunds LH. Postoperative right bundle branch block: identification of three levels of block. Circulation 1980;62:319-28.

48. Motonaga KS, Dubin AM. Cardiac resynchronization therapy for pediatric patients with heart failure and congenital heart disease: A reappraisal of results. Circulation 2014;129:1879-91.

49. Bleeker GB, Kaandorp TAM, Lamb HJ, Boersma E, SteendijkP, Roos A De, et al. Effect of posterolateral scar tissue on clinical and echocardiographic improvement after cardiac resynchronization therapy. Circulation 2006;113:969-76.

50. Auricchio A, Lumens J, Prinzen FW. Does cardiac resynchronization therapy benefit patients with right bundle branch block: cardiac resynchronization therapy has a role in patients with right bundle branch block. Circ Arrhythm Electrophysiol 2014;7:532-42.

51. Plymen CM, Finlay M, Tsang V, O'leary J, Picaut N, Cullen S, et al. Haemodynamic consequences of targeted single- and dual-site right ventricular pacing in adults with congenital heart disease undergoing surgical pulmonary valve replacement. Europace 2015;17:274-80.

52. Vojtovič $P$, Kučera $F$, Kubuš $P$, Gebauer R, Matějka $T$, Tláskal $T$, et al. Acute right ventricular resynchronization improves haemodynamics in children after surgical repair of tetralogy of Fallot. Europace 2018;20:323-8.

53. Janoušek J, Kovanda J, Ložek M, Tomek V, Vojtovič P, Gebauer R, et al. Pulmonary Right Ventricular Resynchronization in Congenital Heart Disease: Acute Improvement in Right Ventricular Mechanics and Contraction Efficiency. Circ Cardiovasc Imaging 2017;10:e006424.

54. Thambo J-B, Guillebon M De, Xhaet O, Santos P Dos, Roubertie F, Labrousse L, et al. Biventricular pacing in patients with Tetralogy of Fallot: non-invasive epicardial mapping and clinical impact. Int $J$ Cardiol 2013;163:170-4.

55. Bordachar P, Iriart X, Chabaneix J, Sacher F, Lafitte S, Jais $P$, et al. Presence of ventricular dyssynchrony and haemodynamic impact of right ventricular pacing in adults with repaired Tetralogy of Fallot and right bundle branch block. Europace 2008;10:967-71.

56. Nanthakumar K, Massé S, Poku K, Silversides CK, Chauhan VS, Mariani JA, et al. Intraoperative High-Density Global Mapping in Adult-Repaired Tetralogy of Fallot. Altered Left Ventricular and Right Ventricular Activation and Implications for Resynchronization Strategies. J Am Coll Cardiol 2010;55:2409-11.

57. Steeg CN, Krongrad E, Davachi F, Bowman FO, Malm JR, Gersony WM. Postoperative left anterior hemiblock and right bundle branch block following repair of Tetralogy of Fallot. Clinical and etiologic considerations. Circulation 1975;51:1026-9. 
58. Thambo J, Santos P Dos, Guillebon M De, Roubertie F, Labrousse L, Sacher F, et al. Biventricular stimulation improves right and left ventricular function after tetralogy of Fallot repair: acute animal and clinical studies. Hear Rhythm 2010;7:344-50.

59. Lumens J, Fan C-PS, Walmsley J, Yim D, Manlhiot C, Dragulescu A, et al. Relative Impact of Right Ventricular Electromechanical Dyssynchrony Versus Pulmonary Regurgitation on Right Ventricular Dysfunction and Exercise Intolerance in Patients After Repair of Tetralogy of Fallot. J Am Heart Assoc 2019;8:e010903.

60. Bussink BE, Holst AG, Jespersen L, Deckers JW, Jensen GB, Prescott E. Right bundle branch block: Prevalence, risk factors, and outcome in the general population: Results from the Copenhagen City Heart Study. Eur Heart J 2013;34:138-46.

61. Link MS, Hill SL, Cliff DL, Swygman CA, Foote CB, Homoud MK, et al. Comparison of frequency of complications of implantable cardioverter- defibrillators in children versus adults. Am J Cardiol 1999;83:263-6. 


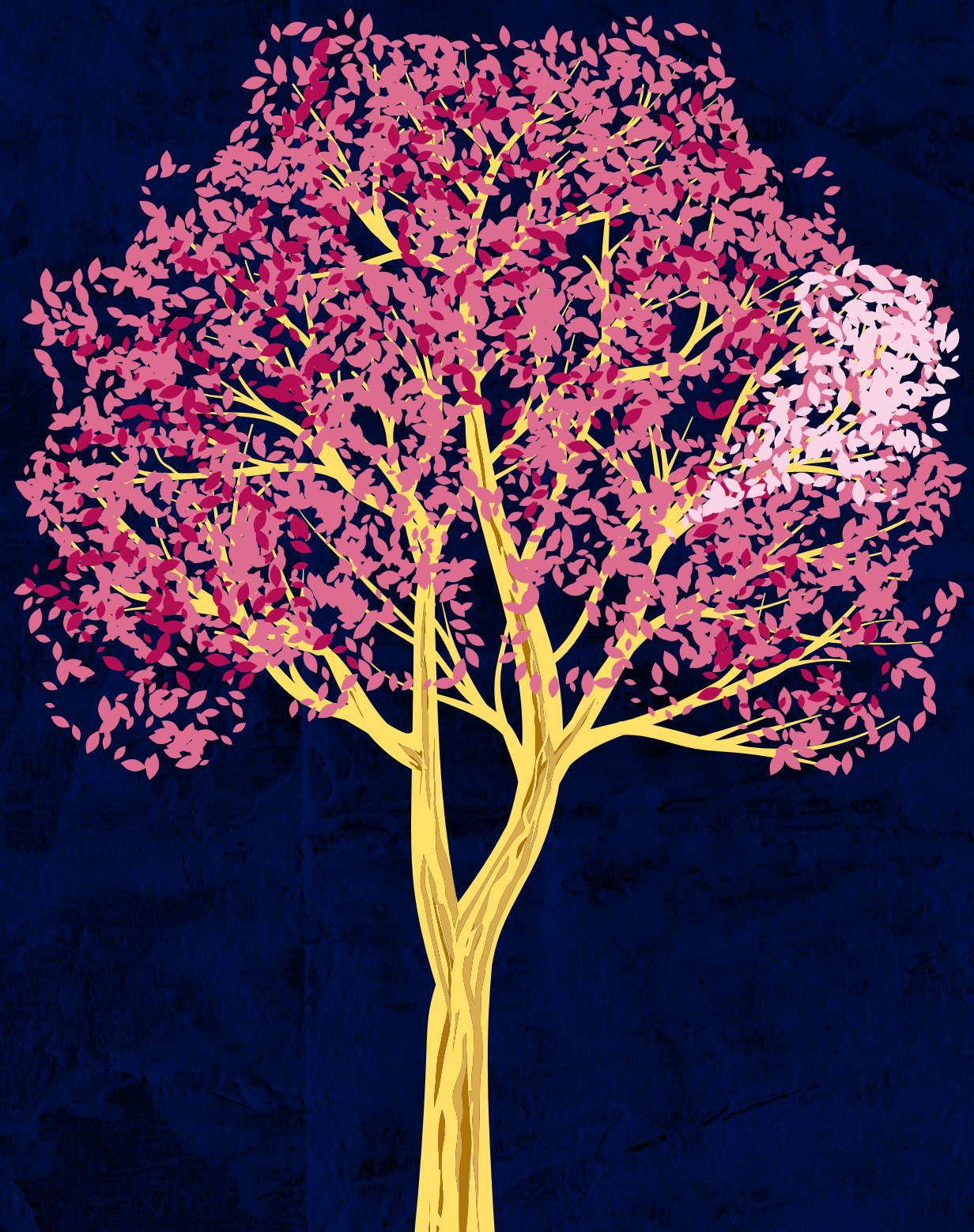


Summary 



\section{Summary}

Normal activation of the cardiac ventricles comes about through a specialized, high-velocity conduction system. The first part of this system is the His bundle, which consists of a left and a right bundle branch. Conduction through these branches enables synchronized and fast activation of both left and right ventricles ( $L V$ and $R V$, respectively).

If one of the bundle branches is damaged, left bundle branch block (LBBB) or right bundle branch block (RBBB) occurs, and activation of the affected ventricle has to come about by means of slower conduction through the working myocardium. For this reason, activation and contraction of the affected ventricle (and in particular, its free wall) are delayed.

Over time, this activation pattern has multiple adverse consequences for the myocardium at the molecular and cellular level (e.g. changes to ion channels related to depolarization and repolarization) and at the organ level (e.g. asymmetric hypertrophy and cardiac dilatation). Progression of all these events leads to worsened cardiac function and more severe conduction delay, starting a vicious circle that can culminate in heart failure.

Cardiac resynchronization therapy (CRT) may reverse this vicious circle. This treatment involves implanting a pacemaker and placing electrodes in the right atrium and in both LV and RV. Resynchronisation through (almost) simultaneous stimulation of both ventricles can cause both acute and long-term improvements in cardiac function, which in turn lead to lower risk of heart failure hospitalisations and death, in particular in patients with heart failure and LBBB.

However, several aspects of this treatment are less well understood. First of all, while the effects of CRT on cardiac depolarization have been studied in considerable detail, much less is known about the effects of CRT on repolarization. Furthermore, much is yet to be learned about the efficacy of CRT in the context of structural heart disease, such as mitral regurgitation (MR) or congenital heart disease. The aim of the research described in this thesis was to branch out into these territories, hopefully yielding deeper understanding of the effects and potential applications of CRT.

\section{Repolarization remodelling during CRT}

Several studies have found that CRT may affect cardiac repolarization. This effect of CRT is particularly important since dispersion of repolarization has been linked to a higher risk of 
cardiac arrhythmias. However, until now, the time course of repolarization remodelling after CRT has not been studied in detail, nor has the relation with contractile remodelling been elucidated.

In chapter $\mathbf{3}$ we explored the effects of CRT on repolarization by measuring $\mathrm{T}$ wave morphology changes after CRT, by relating these changes to mechanical remodelling and by interpreting these results using a patient-specific computational model. We found that changes in repolarization parameters (such as T wave area) already reached a plateau within 5 to 14 days after initiation of CRT. However, some parameters showed contradicting results, which may be explained by the different aspects of repolarization represented by these parameters. Left ventricular ejection fraction and systolic septal strain increased between 2 weeks and 6 months of CRT, suggesting that electrical changes precede mechanical changes.

The observed changes in the $T$ wave were largely reproduced in a patient-specific computer model that assumed an inverse relation between CRT-induced change in activation time and adaptation in action potential duration. The latter changes resulted in a reduction in dispersion of repolarization during chronic CRT as compared to acute CRT.

A more detailed investigation of the link between repolarization remodelling and functional improvement following CRT is described in chapter 4. We analysed vectorcardiograms from 76 patients, both prior to the start of CRT and after six months of treatment. Then, we stratified the patients into two subgroups: those with a T area change smaller (LOW, indicative of relatively little repolarization remodelling) and larger $(\mathrm{HIGH}$, indicative of more repolarization remodelling) than the median T area change. At baseline, the groups demonstrated comparable vectorcardiographic (e.g. QRS and T area) and clinical characteristics. After 6 months, most repolarization parameters were still comparable between the groups, except for $T$ area and amplitude. Patients in the HIGH subgroup showed a larger increase in left ventricular ejection fraction than those in the LOW subgroup, suggesting a link between repolarization remodelling and mechanical changes. However, arrhythmia incidence was low and did not differ between the subgroups. Larger studies are required to confirm the link between T area and left ventricular ejection fraction, and to explore whether there is also a link between repolarization remodelling and clinical improvement or arrhythmia incidence.

\section{CRT during MR}

CRT may beneficially impact MR during ventricular dyssynchrony through several mechanisms. For example, resynchronization of contraction leads to faster build-up of pressure, while resynchronization of the papillary muscles may result in improved positioning of the valve 
leaflets. While both short-term and long-term effects of CRT during MR have been reported, it is not yet clear whether CRT can also be beneficial during structural MR, i.e. MR that is not due to dyssynchrony but due to damage to the mitral valve apparatus. Moreover, it is not known which pacing mode would be most beneficial in this context.

In chapter 5 we explored this topic using an animal model of chronic LBBB+MR. We compared these animals with a control group of animals with only LBBB. Over time, echocardiographic and haemodynamic measurements indicated that LV end-diastolic volume was significantly larger in MR+LBBB dogs than in LBBB animals, while LV end-diastolic pressure also tended to be higher in the former. Therefore, the MR+LBBB model represents a dilated, dyssynchronous cardiomyopathy with MR.

After 16 weeks of LBBB, haemodynamic measurements were performed during three pacing modes: biventricular (BiV) pacing, LV pacing with a short atrioventricular (AV) delay (LV $\left.{ }_{\text {short }}\right)$ and $\mathrm{LV}$ pacing with an $\mathrm{AV}$ delay aimed at fusion with intrinsic activation $\left(\mathrm{LV}_{\text {fusion }}\right)$. All pacing modes significantly increased the maximum rate of LV pressure rise $\left(\mathrm{LVdP} / \mathrm{dt}_{\max }\right)$ in the $\mathrm{LBBB}$ dogs. In the MR+LBBB group BiV pacing tended to increase $\mathrm{LVdP} / \mathrm{dt}_{\max }$ and significantly reduced LV end-diastolic pressure. The different pacing modes had comparable effects.

These data suggest that CRT can be beneficial in LBBB even in the context of structural MR. However, extrapolating results from acute animal studies to long-term clinical effect should be done with care, especially since we did not measure forward blood flow into the aorta.

\section{Conduction properties in patients with repaired Tetralogy of Fallot}

In addition to its beneficial effects on the LV in LBBB, CRT has also been explored in patients with RBBB. A particular situation in which this has been attempted is after surgical repair of Tetralogy of Fallot (ToF), a type of congenital heart disease.

In patients with ToF, a potentially life-threatening combination of abnormalities is present in the heart. Very often, surgery is performed within the first year of life to repair these defects. The complicated repair surgery can lead to RBBB, while pressure and volume overload of the RV may also be present postoperatively.

Patients with repaired ToF (rToF) show both mechanical and electrical dyssynchrony. This suggests that resynchronization of the RV could be beneficial. RBBB is an obvious candidate cause of this dyssynchrony. However, fibrosis due to the combined pressure/volume overload 
or due to surgical scars may also play a role. The distinction between these causes of dyssynchrony is important, since the presence of fibrotic tissue may preclude successful resynchronization through CRT.

In chapter 6 we investigated the cause of cardiac conduction delays in patients with rToF by analysing electrical mapping data that was recorded at Toronto General Hospital. During medically indicated cardiac surgery, more than two decades after ToF repair surgery, cardiac electrical activation was recorded both during sinus rhythm and during ventricular pacing. Thirteen patients with rToF were mapped using an endocardial RV balloon array. Epicardial mapping was performed in 4 of these patients, and also in 2 patients with LBBB and 2 patients without either LBBB or RBBB (non-BBB). We found that structural lines of block were rare in patients with $r$ ToF and that pacing did not increase dispersion of endocardial and epicardial activation of the RV free wall. Moreover, epicardial activation dispersion in both ventricles during pacing was quite comparable for all three groups. This suggests that myocardial conduction properties in rToF patients do not preclude successful resynchronization through CRT.

\section{Conclusions}

Our results indicate that repolarization remodelling after start of CRT occurs rapidly (within 5-14 days) and, based on simulations in a patient-specific computational model, leads to lower dispersion of repolarization. Additionally, more repolarization remodelling may be linked to a better echocardiographic response to CRT.

We also found evidence that CRT still increases LV pump function in the presence of structural MR, even though CRT most likely does not directly influence this kind of MR.

Finally, our studies show that structural conduction blocks are rare in patients with rToF and that tissue properties of the right ventricular free wall are comparable between rToF patients and LBBB or non-BBB patients. 



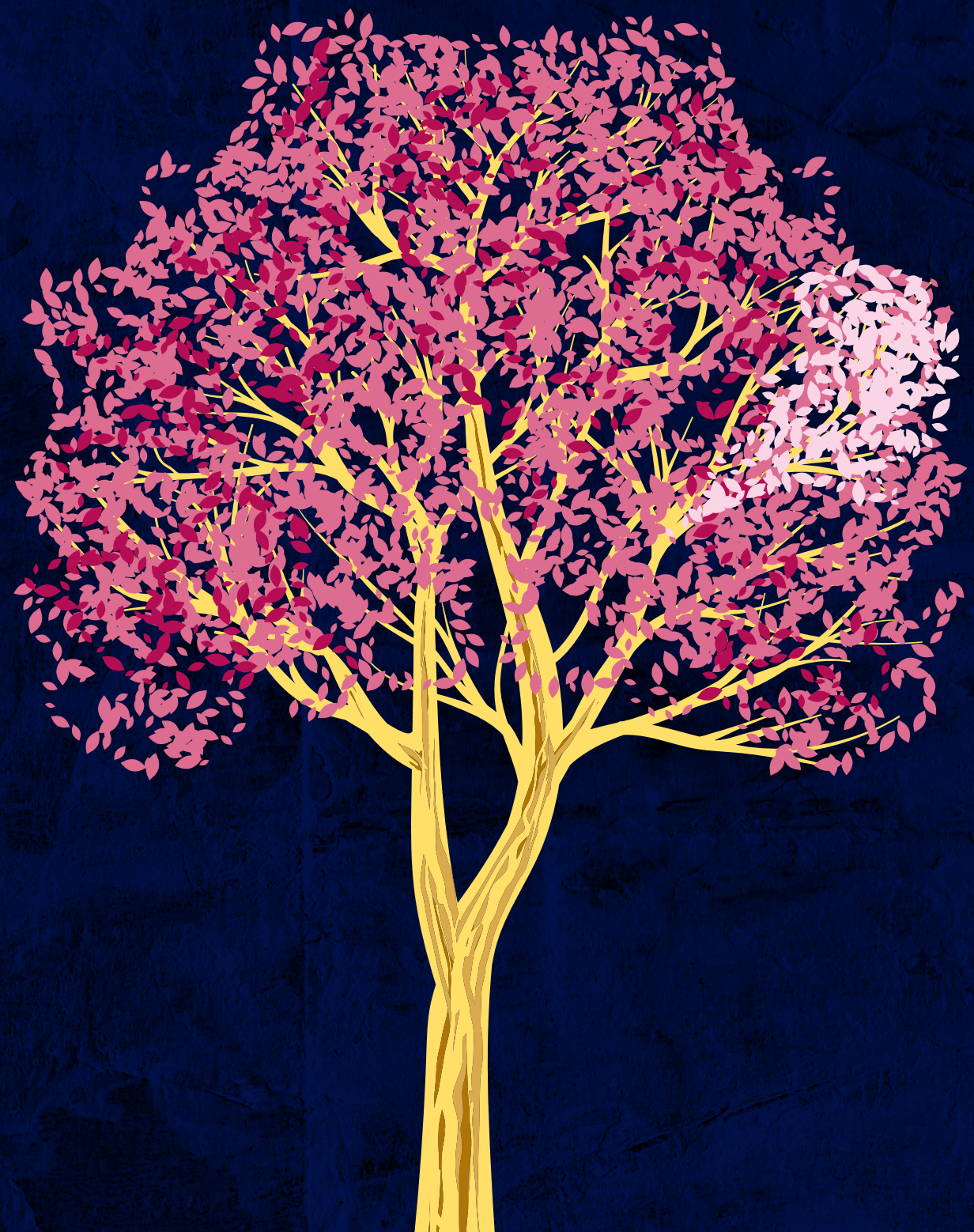


Impact 



\section{Impact}

The main goal of this thesis was to investigate aspects of cardiac resynchronization therapy (CRT) that have not yet been studied in detail. To this end we examined the effects of CRT on ventricular repolarization, as well as the use of this therapy in the context of structural mitral regurgitation (MR) and congenital heart disease.

\section{Scientific impact}

Relatively little is known about changes in the course of repolarization in the context of CRT. Our data provide new information in this area. Note that impulse conduction has attracted most attention in the field of CRT (assessed using the electrocardiogram (ECG) or mapping techniques), but that the processes related to conduction of the depolarization wave are relatively simple. In contrast, myocardial repolarization is an extremely complex process involving multiple ion channels, some of which (e.g. calcium channels) are also related to cardiac contraction. The finding that relatively simple measurements using the (ECG-based) vectorcardiogram provide novel information about repolarization dispersion, in particular during brief interruption of CRT, may open the door to further scientific studies on repolarization processes in the field.

These ECG-based repolarization investigations may be supported by patient-specific modelling. In chapter $\mathbf{3}$ an example of such an approach has been given. The full ECG (QRS complex and T wave) of a patient before start of CRT was fitted to a combined cardiac and thoracic geometrical model with ventricular conduction modelled using the Eikonal approach and simulated ECG based on the lead-field theory. Repeating this approach in a larger cohort may show differences between patients in the pre-CRT repolarization pattern.

The model was also capable of reproducing clinically observed T waves after medium-term follow-up. The change in T wave between the situation before initiation of CRT and after medium-term treatment could be explained by assuming a relation between the change in local activation time (AT) after CRT and the change in local action potential duration (APD) $(\triangle \mathrm{AT}-\triangle \mathrm{APD}$ relation). Repeating these exercises in a larger cohort may provide evidence for interindividual differences in electrophysiological adaptations of the ventricles after CRT that may relate to response to this treatment. The latter studies may be refined by applying locally different $\triangle \mathrm{AT}-\triangle \mathrm{APD}$ relations and by including scar in the model.

On the other hand, the lack of full understanding of the mechanisms of repolarization remodelling calls for more basic scientific studies. These could include electrophysiological 
measurements in cardiomyocytes that underwent long-term stretching. Another approach can be to use computer models with direct coupling of electrophysiological and mechanical properties ${ }^{1}$.

\section{Clinical impact}

Although the studies we performed either involved animals or relatively small numbers of patients, we hope they may be a starting point for new inquiries. One could, for example, study the link between repolarization remodelling and echocardiographic response in a larger population, and also investigate whether there may be a correlation with clinical benefit. The use of more detailed techniques to assess repolarization (e.g. ECG imaging) could be useful in this context. From a functional perspective, it also makes sense to further investigate whether remodelling of repolarization leads to better relaxation with longer-lasting CRT.

In the long term, a better understanding of the different conditions where CRT may be beneficial would be useful. If it were to be proven effective in right bundle branch block, for example, a potentially successful application in larger numbers of patients could be realized.

If repolarization remodelling would prove to be linked to clinical outcome, the relatively short time scale at which repolarization remodelling occurs (approximately 2 weeks) could enable faster fine-tuning of CRT settings, potentially improving its therapeutic effect.

Finally, our findings may contribute to a better understanding of how CRT impacts the heart. This, in turn, may help to find ways to further increase CRT efficacy, which may be advantageous since approximately one third of patients who receive this therapy do not respond ${ }^{2}$. If the mechanism or signalling pathway behind the potential link between electrical and mechanical remodelling were to be elucidated, this might serve as a target for drug development. Enhancing the response to CRT by influencing this electromechanical link could hopefully lead to further improvements in treating cardiac dyssynchrony. 


\section{References}

1. Lyon A, Dupuis LJ, Arts T, Crijns HJGM, Prinzen FW, Delhaas T, et al. Differentiating the effects of $\beta$-adrenergic stimulation and stretch on calcium and force dynamics using a novel electromechanical cardiomyocyte model. Am J Physiol - Hear Circ Physiol 2020;319:H519-30.

2. Daubert JC, Saxon L, Adamson PB, Auricchio A, Berger RD, Beshai JF, et al. 2012 EHRA/HRS expert consensus statement on cardiac resynchronization therapy in heart failure: Implant and follow-up recommendations and management. Europace 2012;14:1236-86. 


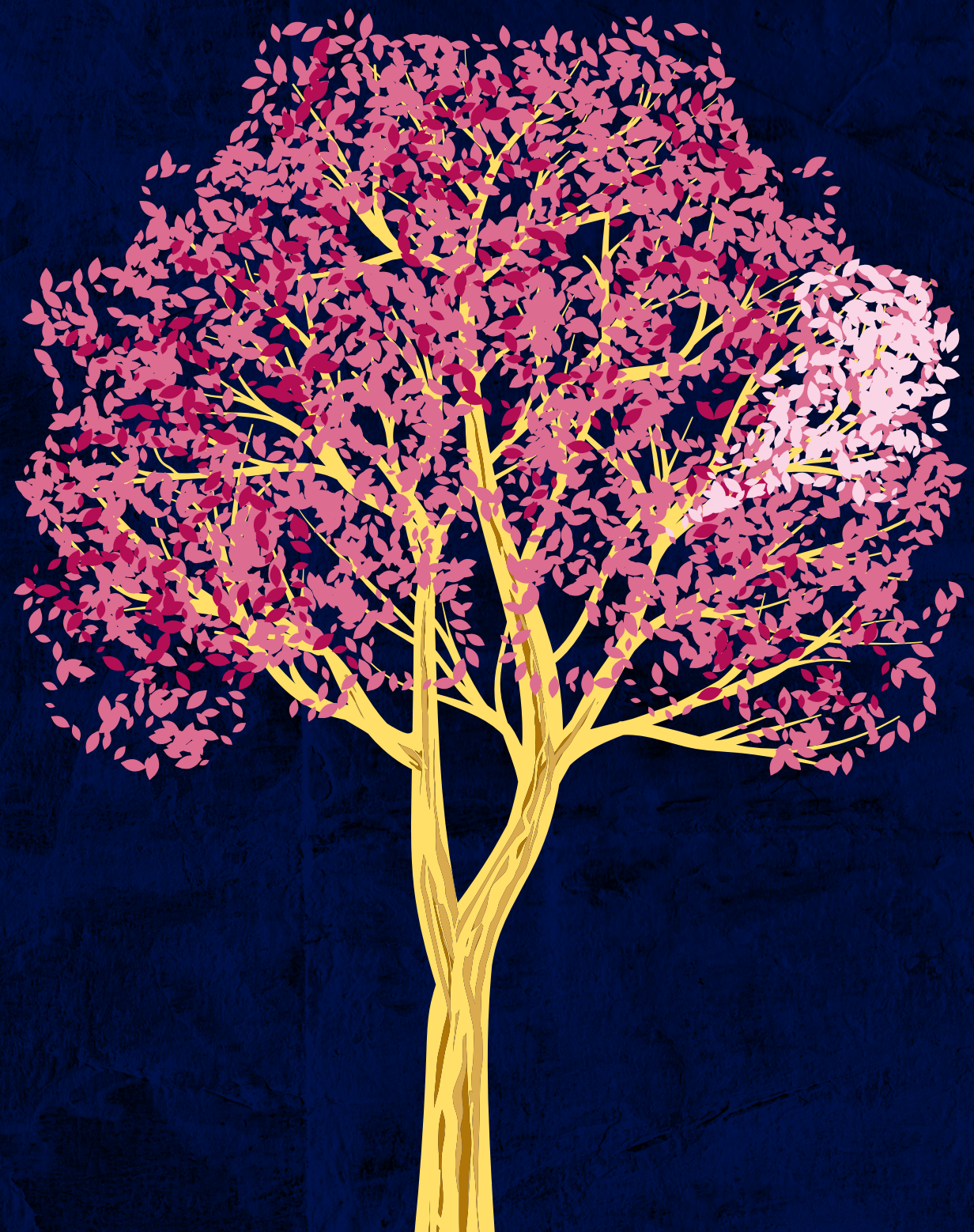


Acknowledgements 



\section{Acknowledgements}

Een proefschrift schrijven is teamwerk. Veel mensen hebben me bijgestaan en begeleid, ideeën aangedragen, geholpen bij experimenten en analyses, een luisterend oor geboden, feedback gegeven en data met me gedeeld. En zonder het samen koffiedrinken, improv spelen, plezier maken, gezellig kletsen, lekkere dingen koken en borrelen was ik er ook zeker niet gekomen. Een klein deel van alle onmisbare mensen staat in deze dank-woordzoeker. Aan hen en aan alle anderen die een bijdrage hebben geleverd: heel, heel veel dank!

Writing a thesis is teamwork. Many people have supported and guided me, contributed ideas, helped with experiments and analyses, shared data, listened patiently and given feedback. And without all the coffee breaks, improv evenings, fun days, chats, dinners and drinks in wonderful company I would have gotten hopelessly stuck. A few of all these indispensable people can be found in this word search of thanks. To them and to all others who have contributed: many, many thanks! 


\begin{tabular}{|c|c|c|c|c|c|c|c|c|c|c|c|c|c|c|c|c|c|c|}
\hline M & $E$ & $\mathrm{~N}$ & É & $S$ & $E$ & $\mathrm{~N}$ & $\mathrm{~T}$ & $G$ & $U$ & $N$ & $\mathrm{E}$ & $R$ & $M$ & $E$ & 1 & K & $E$ & L \\
\hline$U$ & D & V & K & $P$ & $\mathrm{E}$ & $\mathrm{T}$ & $\mathrm{R}$ & A & 1 & $Y$ & 0 & $\mathrm{~T}$ & D & A & C & L & L & $\mathrm{E}$ \\
\hline $\mathrm{J}$ & 0 & $R$ & 1 & $S$ & $\mathrm{~F}$ & $Y$ & V & v & $M$ & 0 & E & D & I & 1 & L & $\mathrm{N}$ & 1 & R \\
\hline B & W & K & B & A & L & $\mathrm{R}$ & $\mathrm{E}$ & I & L & M & A & $\mathrm{N}$ & $\mathrm{R}$ & $\mathrm{E}$ & $\mathrm{N}$ & É & E & 1 \\
\hline A & $\mathrm{N}$ & $\mathrm{R}$ & $\mathrm{J}$ & L & $\mathrm{N}$ & $K$ & A & $\mathrm{F}$ & V & $S$ & 0 & $\mathrm{~T}$ & $\mathrm{H}$ & $\mathrm{F}$ & $\mathrm{R}$ & A & $\mathrm{N}$ & K \\
\hline$M$ & A & $\mathrm{R}$ & 1 & 0 & $\mathrm{~N}$ & D & $K$ & $N$ & D & 1 & A & C & A & E & $\mathrm{T}$ & E & A & A \\
\hline L & $R$ & B & $\mathrm{T}$ & $\mathrm{T}$ & L & U & $\mathrm{R}$ & $\mathrm{R}$ & $S$ & $P$ & A & C & D & $\mathrm{H}$ & $\mathrm{K}$ & $G$ & $G$ & $\mathrm{E}$ \\
\hline A & D & C & $\mathrm{R}$ & V & U & 1 & A & I & $\mathrm{R}$ & $R$ & C & $\mathrm{N}$ & $U$ & C & $U$ & 1 & $\mathrm{~K}$ & $\mathrm{~T}$ \\
\hline R & $\mathrm{H}$ & A & $\mathrm{H}$ & L & L & $\mathrm{H}$ & $\mathrm{J}$ & $U$ & E & 0 & A & $\mathrm{P}$ & $\mathrm{E}$ & z & z & $U$ & $\mathrm{~T}$ & 0 \\
\hline$S$ & B & $\mathrm{N}$ & C & A & C & A & $S$ & $\mathrm{~N}$ & $\mathrm{R}$ & $S$ & E & W & S & L & $P$ & L & B & $\mathrm{E}$ \\
\hline$S$ & C & D & A & 1 & $\mathrm{~N}$ & $\mathrm{R}$ & D & A & $\mathrm{F}$ & 1 & M & A & $\mathrm{T}$ & $\mathrm{T}$ & $\mathrm{H}$ & 1 & $\mathrm{~J}$ & S \\
\hline $\mathrm{T}$ & A & $\mathrm{H}$ & $\mathrm{R}$ & $\mathrm{N}$ & $\mathrm{E}$ & $\mathrm{T}$ & L & I & A & V & C & $\mathrm{T}$ & É & E & $\mathrm{T}$ & A & $\mathrm{H}$ & A \\
\hline $\mathrm{E}$ & $\mathrm{R}$ & R & 0 & D & $\mathrm{T}$ & $\mathrm{R}$ & A & 0 & $M$ & $\mathrm{R}$ & U & C & $\mathrm{P}$ & $F$ & L & 0 & G & S \\
\hline $\mathrm{F}$ & $\mathrm{N}$ & $\mathrm{H}$ & $\mathrm{L}$ & $\mathrm{T}$ & $\mathrm{E}$ & $\mathrm{H}$ & $S$ & L & 1 & 1 & B & $\mathrm{E}$ & $\mathrm{H}$ & $\mathrm{R}$ & C & 1 & $\mathrm{~N}$ & $\mathrm{~K}$ \\
\hline $\mathrm{R}$ & $\mathrm{E}$ & 0 & 1 & $\mathrm{~N}$ & $\mathrm{~T}$ & $\mathrm{~T}$ & A & 1 & $L$ & $\mathrm{E}$ & $\mathrm{R}$ & $\mathrm{E}$ & A & 1 & 0 & $\mathrm{~J}$ & $S$ & 1 \\
\hline 1 & V & A & $\mathrm{N}$ & $\mathrm{E}$ & V & $\mathrm{E}$ & $\mathrm{R}$ & D & I & $\mathrm{N}$ & G & $\mathrm{E}$ & $\mathrm{N}$ & $\mathrm{E}$ & 0 & 0 & W & A \\
\hline $\mathrm{T}$ & $\mathrm{N}$ & $U$ & $\mathrm{E}$ & $M$ & $Y$ & $G$ & $\mathrm{~N}$ & $\mathrm{R}$ & E & D & A & $\mathrm{T}$ & $\mathrm{E}$ & $N$ & V & 0 & A & I \\
\hline S & $\mathrm{R}$ & 1 & C & K & C & 0 & $\mathrm{R}$ & 0 & $N$ & $\mathrm{E}$ & L & $\mathrm{T}$ & U & D & D & S & $\mathrm{R}$ & $\mathrm{E}$ \\
\hline B & 1 & A & $\mathrm{N}$ & C & A & $\mathrm{J}$ & 0 & L & A & $\mathrm{N}$ & D & A & $\mathrm{T}$ & $S$ & 0 & $\mathrm{~T}$ & D & $Y$ \\
\hline 1 & $\mathrm{~N}$ & $G$ & $\mathrm{E}$ & $\mathrm{R}$ & 0 & $\mathrm{R}$ & A & L & $P$ & $\mathrm{H}$ & $U$ & $M$ & $A$ & $\mathrm{R}$ & $\mathrm{T}$ & I & $\mathrm{J}$ & $\mathrm{N}$ \\
\hline
\end{tabular}

Zonder hulp van heel veel . . . . . .

Was dit boekje nooit ......

Ik ben jullie heel erg

' $\mathrm{T}$ is echt waar, het
If these people hadn't . . . . . me

I would not have gotten .... . . .

Please enjoy this tiny $\ldots . .$. .

'Bout ............. 
Promotieteam

FRITS PRINZEN

TAMMO DELHAAS

CAROLINE VAN DEURSEN

\begin{tabular}{ll}
\hline \multicolumn{2}{l}{ Collega's van Fysiologie, in het bijzonder/ } \\
Colleagues from \\
ANysiology, in particular \\
ANDRIES & MARTIJN \\
ARNE & MATTHIJS \\
BEN & MEIKE \\
BIANCA & MOEDI \\
BILLY & PATRICK \\
CHANTAL & RACHELLE \\
ELIEN & RENÉE \\
ELISA & RICHARD \\
FLOOR & RICK \\
FRANS & SANDER \\
GIULIA & STEF \\
JOLANDA & TONI \\
JOLIJN & UYÊN \\
JORIS & VIVIAN \\
LUUK & VLADIMIR \\
MARION & WARD
\end{tabular}

Collega's van BMT, in het bijzonder/

Colleagues from BME, in particular

$\begin{array}{ll}\text { BART } & \text { FRANK } \\ \text { ERIK } & \text { JOOST }\end{array}$

Leden van de beoordelingscommissie

ULI SCHOTTEN

HANS-PETER BRUNNER-LA ROCCA

RUBEN CORONEL

PAUL VOLDERS

MARC VOS

ANGELO AURICCHIO

THIERRY BOVÉ

EUGENE DOWNAR

HUUB

INGER

KEVIN VERNOOY

LARS VAN MIDDENDORP

KUMARASWAMY NANTHAKUMAR

PETRA

SIMONE PEZZUTO

RALPH

SASKIA

STÉPHANE MASSÉ

WOUTER VAN EVERDINGEN

LILIANE WECKE

En, last but certainly not least:

VRIENDEN/FRIENDS

FAMILIE 


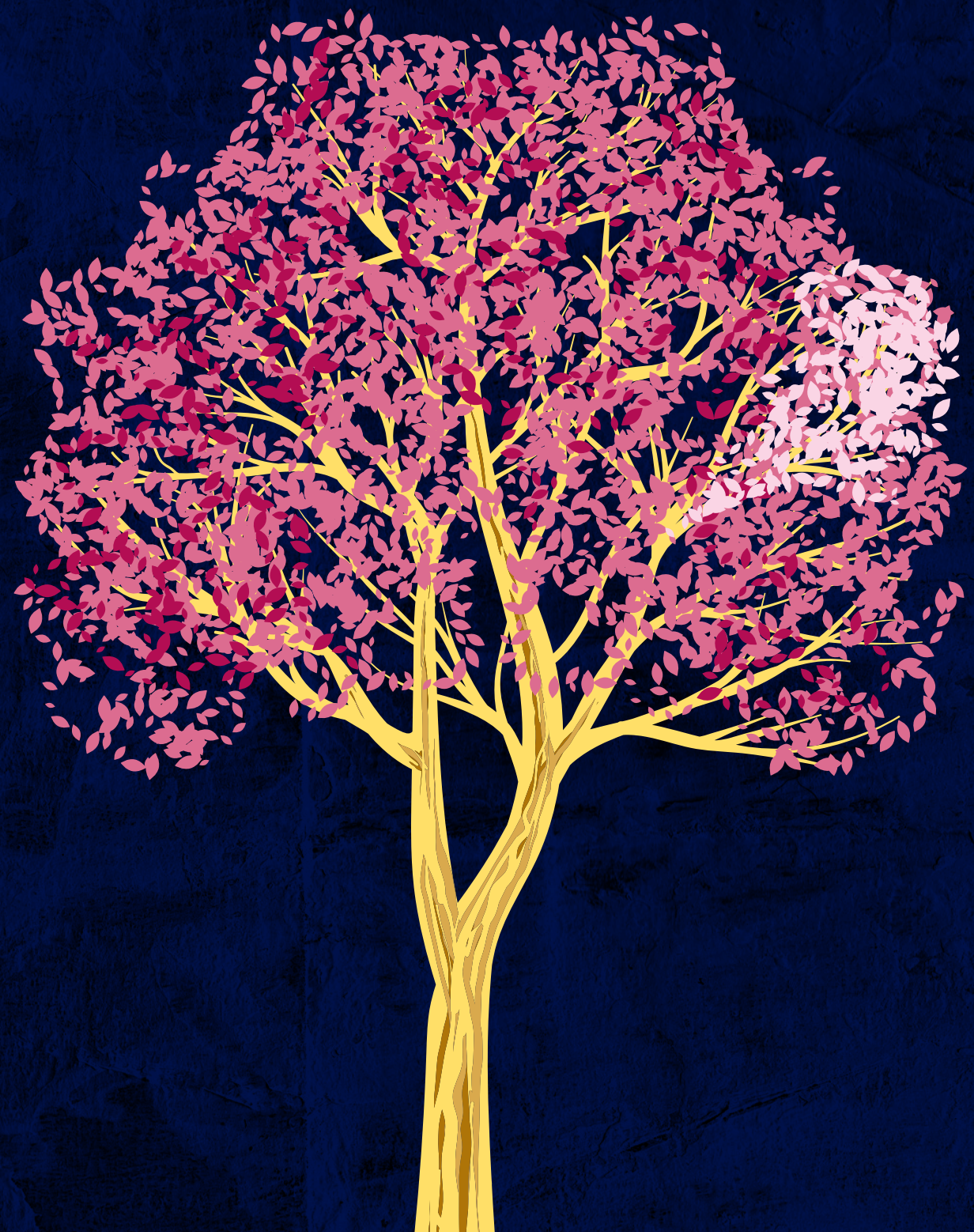


About the author 



\section{About the author}

Nienke Verzaal was born in Haarlem, on the 23rd of March, 1992. She obtained her secondary school diploma at the Kennemer Lyceum in Overveen in 2010 and subsequently moved to Utrecht to study biomedical sciences. During her bachelor's, she took her first steps into cardiovascular research by doing an internship at the laboratory of Experimental Cardiology (University Medical Centre Utrecht, Utrecht).

Nienke started the cardiovascular track of her master's in Biology of Disease by returning to the laboratory of Experimental Cardiology for an internship focusing on the role of EDAfibronectin in adverse cardiac remodelling. Her minor research project took her to the Centre for Molecular Medicine (Karolinska Institute, Stockholm) where she studied the role of the NLRP3 inflammasome in atherosclerosis. Since she not only had an interest in doing science, but also in writing about science, she finished her master's by participating in the science education and communication track and completed an internship at the department of Communication (Amsterdam University Medical Centre - Iocation VUmc, Amsterdam).

In 2016, she started her PhD at the department of Physiology at Maastricht University under supervision of prof. dr. Frits Prinzen, prof. dr. Tammo Delhaas and dr. Caroline van Deursen. The aim of the PhD project was to investigate relatively unexplored facets of cardiac resynchronization therapy (CRT), namely repolarization changes following CRT and the use of CRT in the context of structural mitral regurgitation and congenital heart disease. For this last project, the team in Maastricht cooperated with prof. dr. Kumaraswamy Nanthakumar's research group at The Hull Family Cardiac Fibrillation Management Laboratory (Toronto General Hospital, Toronto). 


\section{Publications}

Nguyên UC*, Verzaal $\mathbf{N J}^{\star}$, Nieuwenhoven FA van, Vernooy K, Prinzen FW. Pathobiology of cardiac dyssynchrony and resynchronization therapy. Europace 2018;20:1898-909.

${ }^{*}$ Authors contributed equally.

Centa M, Jin H, Hofste L, Hellberg S, Busch A, Baumgartner R, Verzaal NJ, et al. Germinal Center-Derived Antibodies Promote Atherosclerosis Plaque Size and Stability. Circulation 2019;139:2466-82.

Verzaal NJ, Massé S, Downar E, Nanthakumar K, Delhaas T and Prinzen FW. Exploring the cause of conduction delays in patients with repaired Tetralogy of Fallot. Europace 2021;23:1105-12. 



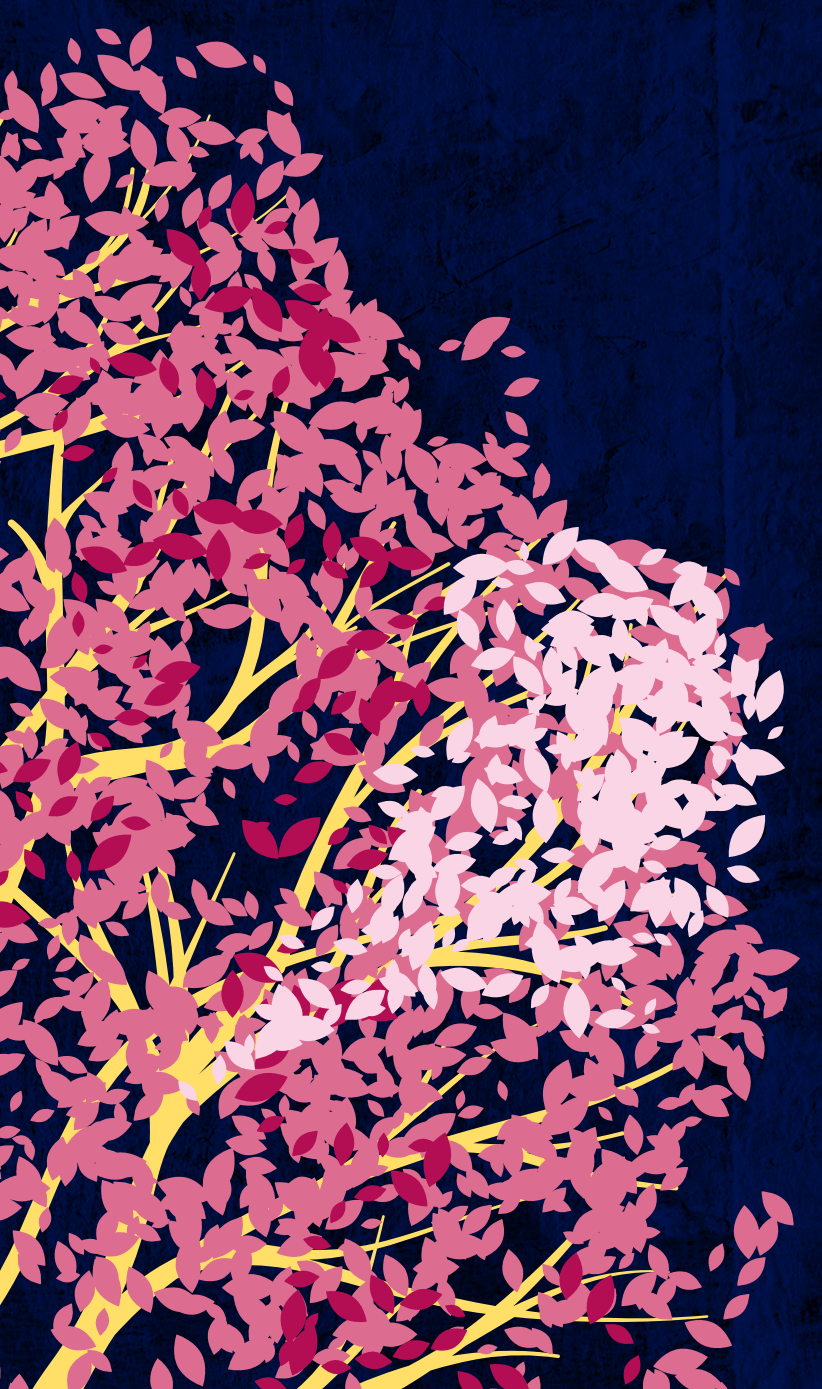

